Aus der Klinik und Poliklinik für Psychiatrie und Psychotherapie der Ludwig-Maximilians-Universität München

Direktor: Prof. Dr. med. Peter Falkai

\title{
Früherkennung der Depression
}

Spezifische Risikofaktoren und Charakteristika der Prodromalphase

\author{
Dissertation \\ Zum Erwerb des Doktorgrades der Humanbiologie \\ An der Medizinischen Fakultät der \\ Ludwig-Maximilians-Universität zu München
}

\author{
vorgelegt von \\ Veronika Stegmüller
}

aus

München

2017 


\section{Mit Genehmigung der Medizinischen Fakultät}

der Universität München

Berichterstatterin:

Mitberichterstatter/in:

Mitbetreuung durch die

promovierte Mitarbeiterin:

Dekan:

Tag der mündlichen Prüfung:
Prof. Dr. med. Eva Meisenzahl

Prof. Dr. Dr. Martin E. Keck

Prof. Dr. med. Dipl.-Psych. Gerd Laux

Dr. rer. med. Juliane Minkwitz

Prof. Dr. med. dent. Reinhard Hickel

01.12 .2017 


\section{Danksagung}

Mein besonderer Dank gilt Frau Prof. Dr. Eva Meisenzahl für die Ermöglichung und ausgezeichnete Betreuung dieser Dissertation, die fachliche Anleitung und uneingeschränkte Unterstützung und Förderung in den letzten zwei Jahren als meine Doktormutter und Mentorin. Ich danke Frau Dr. Juliane Minkwitz für ihre Unterstützung bei der Umsetzung und die vielen anregenden Diskussionen. Weiterhin bedanke ich mich bei Frau Prof. Dr. Frauke Schultze-Lutter, Herrn Prof. Dr. Stephan Ruhrmann und Herrn PD Dr. Cornelius Schüle für den bereichernden wissenschaftlichen Austausch. Herzlichen Dank auch an Herrn Prof. Dr. Wolfgang Wölwer für die kritische Auseinandersetzung mit dieser Arbeit und die konstruktiven Vorschläge. 


\section{Zusammenfassung}

Die vorliegende Studie untersucht die komplexen Entstehungsbedingungen der Depression. Im Rahmen dieser Arbeit wurde ein ätiopathogenetisches Modell zur Früherkennung und Rezidivvorhersage depressiver Erkrankungen entwickelt und daraus ein psychometrisches Messinstrument abgeleitet. Dieses erfasst neben soziodemographischen Variabeln den gesamten Krankheitsverlauf, die klinischen Charakteristika der Prodromalphase ebenso wie zentrale Vulnerabilititätsfaktoren, Stressoren und Ressourcen. Nach Konzeptualisierung eines mehrphasigen Studienvorhabens wurde das entwickelte Inventar DEEP-I (Depression Early Prediction Inventory), bestehend aus Interview und vier Fragebogenbatterien, im Rahmen der hier dargestellten Pilotstudie erstmalig eingesetzt und auf Durchführbarkeit geprüft. Dafür wurden insgesamt 85 Probanden untersucht. Für das entwickelte Messinstrument ergaben sich konstruktive Optimierungsansätze. Im Rahmen der Pilotstudie wurden zudem Risikofaktoren des depressiven Rezidivs sowie die klinischen Charakteristika der Prodromalphase analysiert. In den vorliegenden Daten waren ein rezidivierender Verlauf der Depression und die Anzahl der berichteten Episoden mit einem frühen Ersterkrankungsalter assoziiert. Für den Krankheitsverlauf spielten nach Interpretation der vorliegenden Daten der Familienstand und eine positive Familiengeschichte für psychiatrische Erkrankungen dagegen keine signifikante Rolle. Die durchschnittlich berichtete Prodromalphasendauer der Depression erstreckte sich in der untersuchten Stichprobe im Schnitt über neun Monate. Diese lange Zeitspanne bietet eine bedeutende Chance für sekundärpräventive Maßnahmen. Die klinischen Charakteristika des Prodroms waren hier insbesondere durch somatische Beschwerden, Ängste und Sorgen geprägt. Vereinzelt finden sich dabei gender- und altersspezifische Unterschiede. Dynamische Ätiologiemodelle und eine angepasste Methodik sind für den wissenschaftlichen Fortschritt zur Früherkennung der Depression gefordert. 


\begin{abstract}
The present study examines the complex emergence conditions of depressive diseases. Within the scope of this work, an etiopathological model was developed for the early detection and relapse prediction of depressive disorders and a psychometric measuring instrument was derived from it. In addition to sociodemographic variables, it includes the entire course of the disease, the clinical characteristics of the prodromal phase as well as central vulnerability factors, stressors and resources. After the conceptualization of a multiphase study project, the DEEP-I (Depression Early Prediction Inventory), consisting of an interview and four questionnaire batteries, was used for the first time in the pilot study presented here and tested for feasibility. A total of 85 subjects were examined. For the developed instrument optimization approaches could be derived. Furthermore, risk factors of depressive recurrence as well as the clinical characteristics of the prodromal phase were analyzed. In the present data, a recurrent course of depression and the number of reported episodes were associated with an early age of onset of depression. For the course of the disease, the family status and a positive family history for psychiatric disorders did not play a significant role. The average prodromal phase duration was nine months in the examined sample. This long period of time constitutes a significant opportunity for secondary prevention. The clinical characteristics of the prodrom are characterized in particular by somatic complaints, fears and anxieties. Gender and age-specific differences were found. Dynamic etiology models and an adapted methodology are required for scientific progress in the early detection of depression.
\end{abstract}




\section{Inhaltsverzeichnis}

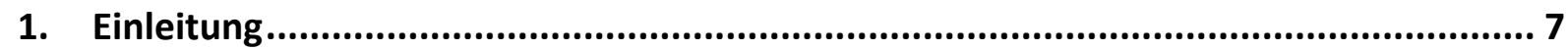

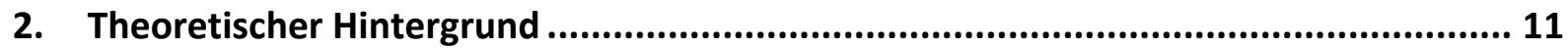

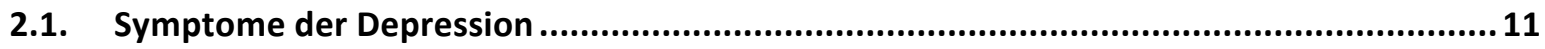

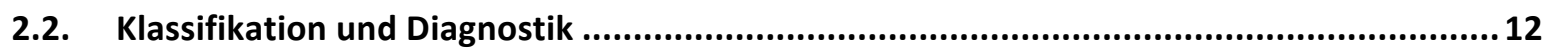

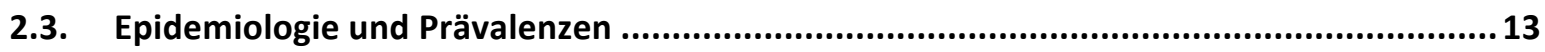

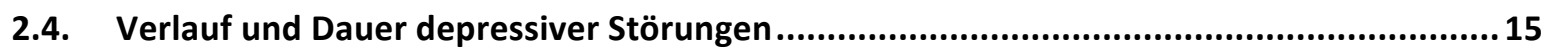

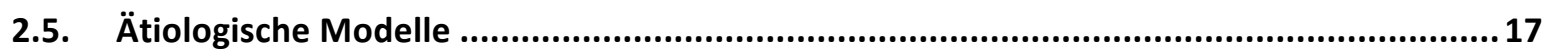

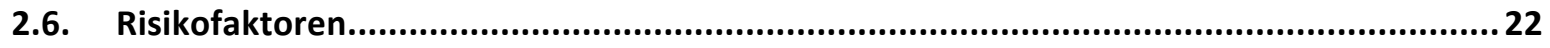

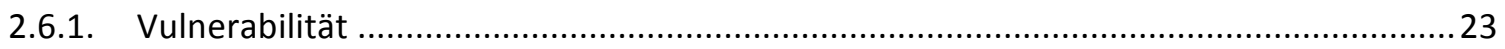

2.6.2. Stressoren: Kritische Lebensereignisse, Arbeits- und Alltagsbelastungen .......................... 34

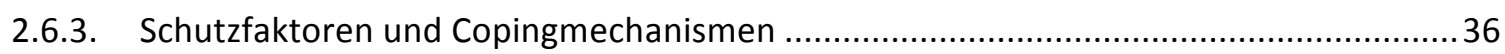

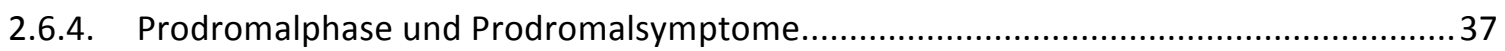

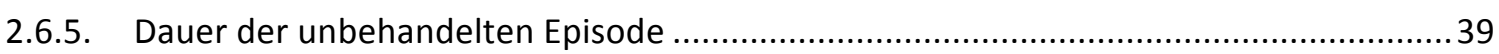

2.6.6. Wechselwirkungen der Risiko- und Schutzfaktoren ................................................... 40

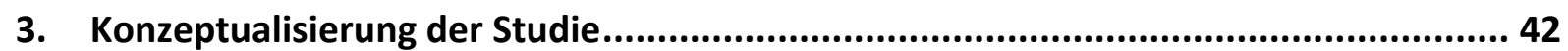

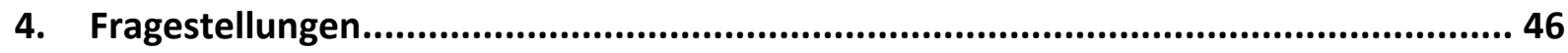

4.1. Risikofaktoren für einen rezidivierenden Verlauf der Erkrankung.................................46

4.2. Charakteristika der Prodromalphase und Dauer der unbehandelten Episode ..................47

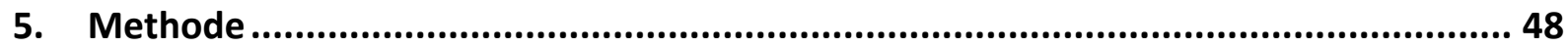

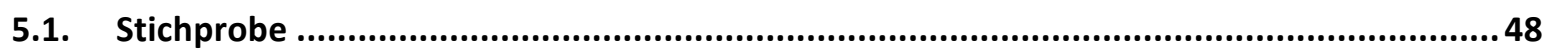

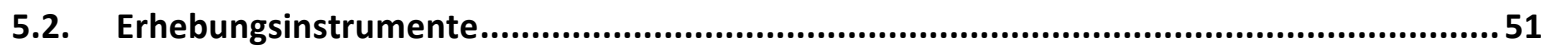

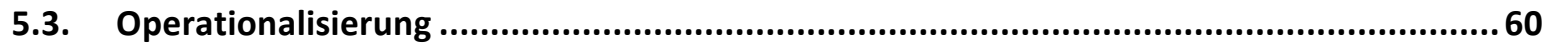

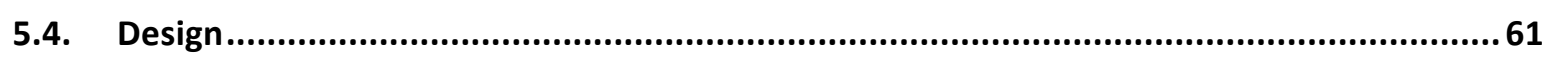

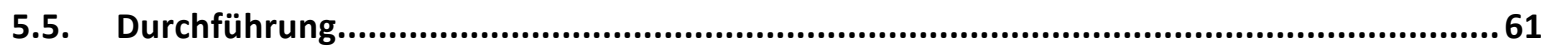

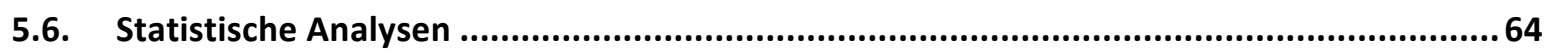

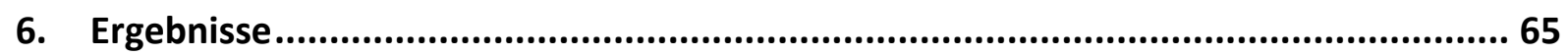

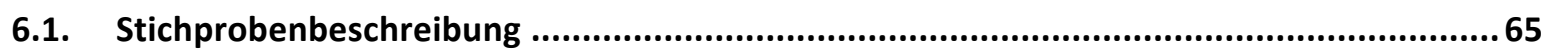

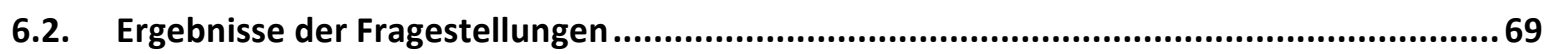

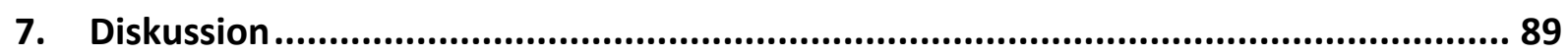

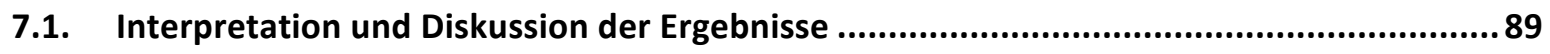

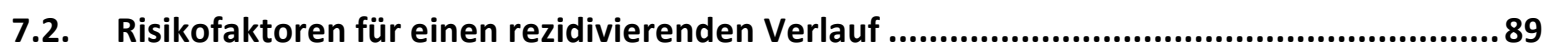

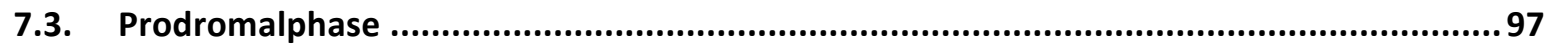

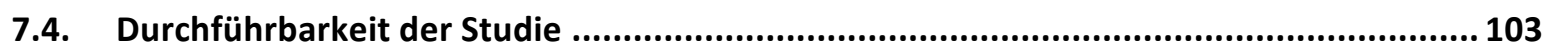

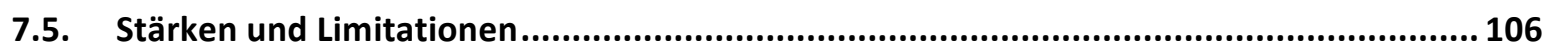

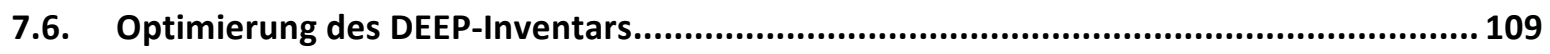

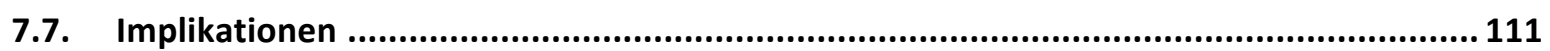

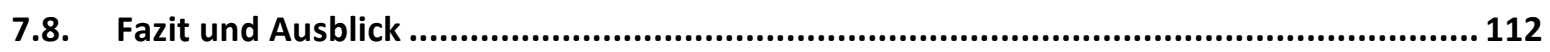

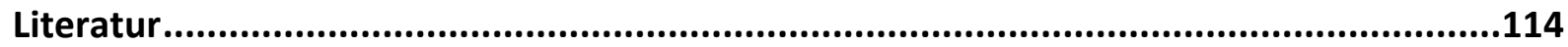

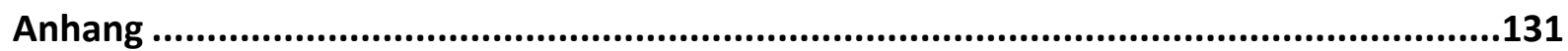




\section{Einleitung}

In der Europäischen Union belaufen sich die jährlichen Kosten verursacht durch psychische Störungen auf etwa 800 Mrd. Euro, den Produktivitätsverlust als wichtigsten Kostenfaktor eingerechnet (Jacobi \& Kessler-Scheil, 2013). Depressionen zählen dabei neben den Angst- und Substanzstörungen zu den häufigsten psychischen Erkrankungen in der Bevölkerung (Wittchen et al., 2012). In Europa stehen sie an erster Stelle der durch krankheitsbedingte Beeinträchtigung verlorenen Lebensjahre (Wittchen, Jacobi, Rehm, Gustavsson, Svensson, Jönsson et al., 2011). Die hohen Erkrankungsraten, die kostenintensiven Folgen und die Mortalität depressiver Störungen erfordern unabdingbar eine Reduktion von Inzidenz- und Prävalenzraten. Zukünftig stellt dies eine zentrale Aufgabe für die öffentliche Gesundheit dar. Gefordert sind aber nicht nur ein rasches Erkennen der Depression und die zeitnahe Einleitung einer adäquaten und leitliniengerechten Behandlung, sondern auch die richtige Einschätzung eines Hochrisikozustandes einer Person im Sinne der Früherkennung. Konkret bedeutet dies, Personen zu identifizieren, welche in nächster Zeit mit hoher Wahrscheinlichkeit an einer Depression erkranken werden, um sie im Anschluss entsprechenden Interventionen zuzuführen. Die Senkung der Erkrankungsraten kann dabei über die Etablierung gezielter Primär- und Sekundärpräventionsmaßnahmen realisiert werden (Ferrari et al., 2013). Entsprechende Maßnahmen zur gezielten Früherkennung der Depression sind bislang jedoch weder im Leistungsspektrum ärztlicher Versorgung enthalten noch existieren dafür geeignete Strukturen im deutschen Gesundheitssystem. Hinzu kommt, dass eine leitliniengerechte Behandlung mit antidepressiver Medikation oder Psychotherapie nur bei einem Bruchteil der Betroffenen erfolgt (Wittchen, Jacobi, Klose \& Ryl, 2010).

Zur Senkung der Erkrankungsraten sind demnach zwei Elemente wesentlich: Die gezielte Früherkennung der Depression kombiniert mit der Etablierung von Sekundärpräventionsmaßnahmen sowie die Verbesserung der Diagnostik und Behandlungseinleitung für bereits Erkrankte. Sowohl depressiv Erkrankte als auch Personen, die ein hohes Erkrankungsrisiko aufweisen, werden oft nur unzureichend therapeutisch versorgt. Als Ursache dafür werden nach wie vor Stigmatisierung, Fehleinschätzungen des Schweregrads der Erkrankung, diagnostische Defizite und Versorgungsengpässe diskutiert (Hegerl, Rummel-Kluge \& Heinz, 2016). Bei psychischen Beschwerden ist in vielen Fällen die hausärztliche Anbindung die erste Anlaufstelle für Diagnostik und Einleitung einer 
Behandlung (Kivelitz, Watzke, Schulz, Harter \& Melchior, 2015). Der allgemeinärztliche Kontakt ist damit die Schlüsselstelle in der Früherkennung und Erstversorgung. Um dies jedoch optimal nutzen zu können, müssen insbesondere die Erkennungsraten und die Diagnostik sowie die Vernetzung der primärärztlichen mit der fachärztlichen Versorgung verbessert werden (Sikorski, Luppa, König, van den Bussche \& Riedel-Heller, 2012). Einer Metaanalyse von Mitchell, Vaze und Rao (2009) zufolge liegt die Sensitivität in der Diagnostik der Depression im allgemeinärztlichen Setting bei etwa 50\%. Nur rund die Hälfte aller Erkrankten wird demnach als solche überhaupt erkannt, obwohl standardisierte Messinstrumente zur Diagnostik vorliegen. Diese Schwierigkeiten in der diagnostischen Einschätzung verhindern eine adäquate Behandlungseinleitung und die Gefahr der Chronifizierung steigt. Anzunehmen ist hier, dass die Erkennung subklinischer Depressionen oder gar eines Hochrisikozustandes für eine depressive Erkrankung noch seltener gelingt, zumal geeignete diagnostische Mittel zur Früherkennung bislang fehlen (Patten, 2013). Zusätzlich ist in der primärärztlichen Versorgung die Früherkennung der Depression keine von den Versicherungen getragene Leistung. Sie stellt damit aus Kostengründen, aufgrund fehlender zeitlicher Ressourcen und geringer Spezialkenntnisse auf dem Gebiet der psychiatrischen Früherkennung eine große Herausforderung für die Behandler im hausärztlichen Setting dar. Eine effektive und ökonomische Prävention kann jedoch nur auf Grundlage einer adäquaten Risikoeinschätzung, im Sinne der Identifikation von HochrisikoPersonen und differenzierbaren Prodromalzuständen erfolgen. Daraus ergibt sich die Forderung nach einer Operationalisierung des Prodromalzustandes der Depression. Der erste Schritt dahingehend muss die Entwicklung eines Verfahrens sein, welches in Allgemeinarztpraxen, Beratungsstellen oder Früherkennungszentren eingesetzt werden kann, um Personen mit einem erhöhten Erkrankungsrisiko zu identifizieren und auf dessen Grundlage dann gegebenenfalls spezifische und individualisierte Präventionsmaßnahmen eingeleitet werden können. Ziel ist es, den Schweregrad und die Dauer der Erkrankung abzumildern oder deren Ausbruch gänzlich zu verhindern. Die multifaktorielle Genese und das heterogene Erscheinungsbild der Depression erschweren jedoch eine reliable Konzeption und operationalisierbare Klassifikation von Hochrisikozuständen. Eine differenzierte Erfassung und Unterscheidung von Risiko- und Schutzfaktoren sowie Phänotypen der Depression ist dafür notwendige Voraussetzung. 
Bislang existieren nur wenige Forschungsvorhaben mit dem Ziel der Prädiktion depressiver Erkrankungen. Im europäischen Raum entwickelten King et al. (2008) auf Basis einer Kombination bisher erforschter Risikofaktoren depressiver Erkrankungen einen prognostischen Algorithmus, welcher das Erkrankungsrisiko prozentual für einen bestimmten Zeitraum vorhersagen soll. Der Algorithmus umfasst insgesamt 39 Risikofaktoren, welche in einer prospektiven Studie auf prognostische Validität untersucht wurden (Bellón et al., 2011). Unter den erfassten Variablen und Risikofaktoren fanden sich unter anderem Geschlecht, sozioökonomischer Status, frühere Episoden depressiver Erkrankungen, eine positive Familiengeschichte für psychische Erkrankungen und Suizid, kritische Lebensereignisse, der allgemeine physische Gesundheitszustand, Missbrauchserfahrungen in der Kindheit, die Qualität sozialer Kontakte, die Qualität der Partnerschaft, Alkohol- und Drogenkonsum, Religiosität/Spiritualität, Diskriminierungserfahrungen und soziale Unterstützung. Die genannten Risikofatoren können unter den Konstrukten Vulnerabilität und Stressoren zusammengefasst werden. Den Autoren zufolge ist der Risikoalgorithmus vergleichbar einsetzbar wie etablierte Risikokalkulationsverfahren etwa aus dem Bereich kardiovaskulärer Erkrankungen (King et al., 2008). Auffallend in der Konzeption von King und Kollegen (2008) ist jedoch das Fehlen von Persönlichkeitsmerkmalen, Schutzfaktoren im Sinne von Resilienz und Ressourcen sowie psychopathologischen Charakteristika, mit Ausnahme von Angstsymptomen. Diese Faktoren sind jedoch, wie in zahlreichen Studienergebnissen nachgewiesen, häufig an der Entstehung depressiver Erkrankungen beteiligt (siehe Alvaro, Roberts \& Harris, 2013; Kotov, Gamez, Schmidt \& Watson, 2010; Monteiro, Fernandes da Silva, Pereira \& Vaz Serra, 2011). Empirische Untersuchungen konnten beispielsweise zeigen, dass in der Prodromalphase einer Depression bereits psychopathologische Veränderungen nachweisbar sind, die mit einem späteren Erkrankungsrisiko assoziiert sind (Fava, Grandi, Canestrari \& Molnar, 1990; Fava \& Tossani, 2007; Georgiades, Lewinsohn, Monroe \& Seeley, 2006; Keenan et al., 2008; Syed Sheriff, McGorry, Cotton \& Yung, 2015). Ein Prädiktionsalgorithmus sollte demnach neben den bereits genannten Risikofaktoren aus den Bereichen der Stressoren und Vulnerabilität auch die psychopathologischen Veränderungen der Prodromalphase sowie Schutzfaktoren und Persönlichkeitsmerkmale einbeziehen. In einer aktuellen Studie konnten auf Basis retrospektiver Selbstberichte über Charakteristika der Erstmanifestation einer Depression die Dauer, Chronizität und der Schweregrad der Erkrankung in prädiktiven 
Modellen vorhergesagt werden (Kessler et al., 2016). Dieser Befund spricht für die Nutzbarkeit retrospektiv berichteter psychopathologischer Charakteristika zur Prädiktion von depressiven Rezidiven. Analog dazu lässt sich vermuten, dass auch die klinischen Charakteristika der Prodromalphase eine prognostische Validität bezüglich depressiver Erkrankungen aufweisen und in ein multifaktorielles Prädiktionsmodell aufgenommen werden sollten. Studien belegen eine Manifestation von Prodromalsymptomen schon Wochen oder Monate vor Beginn der eigentlichen Erkrankung (Fava \& Kellner, 1991; Fava \& Tossani, 2007). Die Erfassung dieser psychopathologischen Auffälligkeiten könnte dazu beitragen, Rückfälle früher und effektiver behandeln zu können oder gar den Ausbruch einer Depression zu verhindern (Fava \& Kellner, 1991).

Ziel des vorliegenden Forschungsvorhabens ist die Konzeption eines mehrdimensionalen Entstehungsmodells der Depression, auf dessen Basis die Früherkennung und die Rezidivvorhersage operationalisiert werden können. Dieses Modell soll im Gegensatz zu bisher beschriebenen Modellen neben Vulnerabilitätsfaktoren und Stressoren auch Persönlichkeitsaspekte, Schutzfaktoren und psychopathologische Veränderungen des Prodroms integrieren. Daraus soll ein Instrumentarium abgeleitet werden, welches eine individualisierte Risikokalkulation der Depression erlaubt und die Einleitung spezifizierter Präventivprogramme ermöglicht. In der vorliegenden Arbeit sollen die erfolgte Modellbildung und Studienkonzeptualisierung dargestellt sowie erste Ergebnisse einer Pilotstudie vorgestellt werden. 


\section{Theoretischer Hintergrund}

\subsection{Symptome der Depression}

Nach dem Internationalen Klassifikationssystem psychischer Störungen (ICD-10; Dilling, Mombour \& Schmidt, 2014) ist die Depression gekennzeichnet durch die Leitsymptome gedrückte Stimmung, Interessen und/oder Freudverlust und Antriebsminderung. Als weitere häufige Symptome des depressiven Syndroms werden verminderte Konzentration und Aufmerksamkeit, vermindertes Selbstwertgefühl und Selbstvertrauen, Schuldgefühle, Gefühle der Wertlosigkeit, negative und pessimistische Zukunftsperspektiven, Schlafstörungen, verminderter Appetit sowie Suizidgedanken aufgeführt. Zudem treten häufig körperliches Unwohlsein, Ängstlichkeit und erhöhte Ermüdbarkeit auf. Es handelt sich um eine Störung des gesamten Organismus (Beesdo-Baum \& Wittchen, 2011). Die Lebensqualität und die sozialen Belange sind meist stark beeinträchtigt (Wittchen \& Jacobi, 2006). Die Symptome variieren jedoch meist interindividuell beträchtlich (Margraf \& Schneider, 2009), was die Diagnostik und damit auch die Früherkennung erschwert. Im Allgemeinen können die Symptome unterschiedlichen Ebenen zugeordnet werden. Charakteristisch ist hier eine Einteilung in emotionale, kognitive, physiologische und behaviorale/motorische Veränderungen (Beesdo-Baum \& Wittchen, 2011). Neuere Forschungsergebnisse stellen zudem eine genderspezifische Symptomatik der Depression zur Diskussion, nach der die gängigen Klassifikationssysteme eher weibliche Attribute der Depression abbilden, wie zum Beispiel Traurigkeit und emotionale Labilität (Martin, Neighbors \& Griffith, 2013). Studienergebnisse zu geschlechterspezifischer Psychopathologie lassen darauf schließen, dass die Kernsymptome der Depression sich bei Männern und Frauen zwar nicht unterscheiden, Männer im Allgemeinen aber weniger Symptome berichten als Frauen und im Rahmen der Depression häufiger Ärger, Aggressionen, Substanzabusus und risikoreiches Verhalten aufweisen als Frauen (Möller-Leimkühler, 2008). Bezüglich der Psychopathologie der Prodromalphase kann dahingehend ebenfalls eine unterschiedliche Symptompräsentation bei Männern und Frauen erwartet werden. Studien existieren diesbezüglich bisher nicht.

Forschung zu den Unterschieden depressiver Symptome bei älteren und jüngeren Menschen zeigt eine zum Teil unterschiedliche Phänomenologie depressiver Erkrankungen für verschiedene Altersgruppen. Am prägnantesten stellt sich dabei eine verstärkte 
Präsentation somatischer Symptome im Rahmen depressiver Erkrankungen bei älteren Betroffenen im Vergleich zu jüngeren Erkrankten dar (Hegeman, Kok, van der Mast \& Giltay, 2012). Die Ergebnisse der genannten Metaanalyse liefern dahingehend wichtige Implikationen für die Früherkennung der Depression, da auch eine phänomenologisch unterschiedliche Prodromalsymptomatik in den verschiedenen Altersgruppen zu erwarten ist.

\subsection{Klassifikation und Diagnostik}

Im ICD-10 werden die depressiven Störungen in der Sektion der affektiven Störungen klassifiziert. Unterschieden werden dabei die manische Episode, die bipolar affektive Störung, die depressive Episode, die rezidivierende depressive Störung, die anhaltende affektive Störung und andere affektive Störungen sowie nicht näher bezeichnete affektive Störungen. Das diagnostische und statistische Manual psychischer Störungen (DSM-5; Falkai \& Wittchen, 2015) unterscheidet in der Sektion depressive Störungen hier die Major Depression, die persistierende depressive Störung (Dysthymie), die prämenstruelle dysphorische Störung, die substanz-/medikamenteninduzierte depressive Störung, die depressive Störung aufgrund eines anderen medizinischen Krankheitsfaktors, die andere näher bezeichnete depressive Störung und nicht näher bezeichnete depressive Störungen. Die diagnostische Einordnung berücksichtigt in beiden Klassifikationssystemen den aktuellen Schweregrad der Symptome und den Krankheitsverlauf (Beesdo-Baum \& Wittchen, 2011). Eine depressive Episode (ICD-10) oder Major Depression (DSM-5) wird nach den genannten gängigen Klassifikationssystemen dann diagnostiziert, wenn mindestens vier der genannten Symptome, davon zwingend zwei der Kernsymptome (gedrückte Stimmung und/oder Freudund Interessenverlust und/oder Antriebsmangel), gleichzeitig über einen Zeitraum von mindestens zwei Wochen bestehen und damit eine Beeinträchtigung der Funktionsfähigkeit einhergeht (Hautzinger, 2015a). Die Voraussetzung der Diagnosestellung ist die Persistenz der Symptome, die Überschreitung einer bestimmten Intensität und eine zeitliche Mindestdauer von zwei Wochen. In Deutschland erfolgt die Diagnosestellung nach der Verschlüsselung des ICD-10. Die diagnostischen Kriterien einer depressiven Episode nach ICD-10 sind zur Übersicht in Tabelle 1 aufgeführt. 
Tabelle 1

Diagnostische Kriterien einer depressiven Episode nach ICD-10

Für die Diagnose sollten mindestens zwei der drei unter 1. aufgeführten Symptome vorhanden sein, sowie mindestens zwei der unter 2. aufgeführten Symptome. Die Mindestdauer beträgt zwei Wochen.

1. - Depressive Stimmung

- Verlust von Freude oder Interesse

- Antriebsmangel, erhöhte Ermüdbarkeit

2. - Verminderte Konzentration und Aufmerksamkeit

- Vermindertes Selbstwertgefühl und Selbstvertrauen

- Schuldgefühle und Gefühle der Wertlosigkeit

- Negative und pessimistische Zukunftsperspektiven

- Schlafstörungen

- Verminderter Appetit

- Suizidgedanken, erfolgte Selbstverletzung oder Suizidhandlungen

\subsection{Epidemiologie und Prävalenzen}

Das Risiko im Laufe des Lebens an einer psychischen Störung zu erkranken beträgt nach Kessler et al. (2005) in etwa 50\%. Depressionen gehören dabei zu den weltweit häufigsten psychischen Erkrankungen (Jacobi \& Kessler-Scheil, 2013). Die Anzahl der Erkrankten ist epidemiologischen Studien zufolge in den letzten Jahren gestiegen, wobei immer jüngere Altersgruppen betroffen sind (Beesdo et al., 2009). Die Schätzung der Prävalenzraten variiert dabei beträchtlich für die einzelnen Länder (Kessler, 2012; Moffitt et al., 2010). Nach Metaanlysen zu den Prävalenzraten rangieren die Schätzungen der Lebenszeitprävalenz für Erwachsene je nach Studie zwischen 15 und 47\% (Hamdi \& lacono, 2014; Moffitt et al., 2010; Olino et al., 2012). Die durchschnittliche Schätzung der Lebenszeitprävalenz einer Major Depression liegt in Nordamerika bei 16.6\% (Kessler et al., 2005). Die durchschnittliche 
Lebenszeitprävalenz für Frauen wird hier mit 22.9\%, für Männer mit 15.1\% berichtet. Diese Ergebnisse decken sich mit jüngsten Studienergebnissen aus dem europäischen Raum (Uhmann \& Wittchen, 2010; Wittchen, Jacobi, Rehm, Gustavsson, Svensson, Jonsson et al., 2011). Die 12-Monatsprävenlenz für die Major Depression oder depressive Episode liegt in Deutschland neuesten Erhebungen zufolge bei $6.8 \%$ in der erwachsenen Allgemeinbevölkerung (Jacobi et al., 2015). Die Schätzung der kumulativen Inzidenz liegt bei 29.6\% für die Altersgruppe der 33-Jährigen (Beesdo et al., 2009). In der Altersgruppe der 3349-Jährigen finden sich auch die höchsten Lebenszeitprävalenzen mit 26.7\% für Frauen und 18.6\% für Männer. Die niedrigsten Schätzungen ergaben sich hier in der Altersgruppe der über 65-Jährigen mit 13.0\% für Frauen und 5.3\% für Männer (Uhmann \& Wittchen, 2010). Die empirischen Daten sprechen insgesamt für einen allgemeinen Anstieg der Erkrankungsraten (siehe Hidaka, 2013). Insbesondere in den jüngeren Geburtskohorten scheinen die Prävalenzraten und das Erkrankungsrisiko deutlich zu steigen (Beesdo-Baum \& Wittchen, 2011; Uhmann \& Wittchen, 2010). Erklärungsmodelle diesbezüglich reichen von veränderten gesellschaftlichen Bedingungen, circadianen Umstellungen und Schlafdeprivation bis hin zu Übergewicht und veränderten Ernährungsgewohnheiten in der heutigen Gesellschaft (Hidaka, 2013). In der Literatur wird diesbezüglich häufig auch die undifferenzierte diagnostische Erfassung der Depression in den verschiedenen Altersgruppen diskutiert (Knäuper \& Wittchen, 1994).

Die dargestellte Befundlage epidemiologischer Untersuchungen zu den Prävalenzzahlen lässt verschiedene ätiopathogenetische Mechanismen der Depression für unterschiedliche Altersgruppen sowie für Männer und Frauen vermuten. Die Ergebnisse prospektiver Longitudinalstudien zeigen, dass Depressionen schon im Jugendalter häufig zu beobachten sind (Hankin et al., 1998) und sich die Prävalenzen depressiver Erkrankungen für Jungen und Mädchen ab einem Alter von etwa 14 Jahren zu unterscheiden beginnen (Uhmann \& Wittchen, 2010). Wissenschaftlichen Untersuchungen zufolge erkranken Frauen etwa doppelt so häufig wie Männer an Depressionen (Möller-Leimkühler, 2008). Neuere Theorien zu diesen sehr deutlichen Unterschieden in den Prävalenzraten beziehen sich vor allem auf die Annahme unterdiagnostizierter und unterbehandelter depressiv erkrankter Männer. Als Gründe dafür sind nach Möller-Leimkühler (2008) drei Faktoren zu nennen: Das weniger ausgeprägte Hilfesuchverhalten bei Männern, dysfunktionale Stressverarbeitungsmuster sowie ein Gender-Bias in der Diagnostik. Bei Berücksichtigung der oben genannten 
Unterschiede auf Symptomebene zwischen Männern und Frauen konnte eine Angleichung der Prävalenzraten von Männern und Frauen beobachtet werden (Martin et al., 2013). Die Übertragung der dargestellten Befunde auf die Früherkennung der Depression würde eine differenzierte Erfassung der Prodromalsymptome und des Hilfesuchverhaltens für Männer und Frauen implizieren. Das Hilfesuchverhalten hat möglicherweise einen direkten Einfluss auf die Dauer der Prodromalphase und die Dauer der unbehandelten Erkrankung (DUE), was wiederum einen spezifischen Einfluss auf das Erkrankungsrisiko und den Verlauf der Depression nehmen kann (Ghio et al., 2015).

Die dargestellten Befunde zur Phänomenologie und den Prävalenzen der Depression machen die Schwierigkeit der Diagnostik und Früherkennung deutlich. Zusammenfassend lässt sich daraus schlussfolgern, dass die Früherkennung der Depression spezifisch und differenziert für Männer und Frauen sowie für unterschiedliche Altersgruppen angesetzt werden sollte. In der Konsequenz muss eine Risikoprofilerstellung demnach gender- und altersgruppenspezifisch erfolgen.

\subsection{Verlauf und Dauer depressiver Störungen}

Ein weiterer bedeutsamer und für die Früherkennung der Depression zu berücksichtigender Aspekt ist der Verlauf depressiver Störungen. Affektive Erkrankungen verlaufen in der Regel episodisch. Man unterscheidet dabei zwischen einer einzelnen depressiven Episode, einem chronischen Verlauf einer einzelnen Episode und einer rezidivierenden depressiven Störung mit oder ohne Vollremission im Intervall. Weiterhin wird in den gängigen Diagnosesystemen zwischen der dysthymen Störung, der so genannten Double Depression im Sinne einer episodisch überlagerten dysthymen Störung, einer zyklothymen Störung und der bipolaren Störungen differenziert (Beesdo-Baum \& Wittchen, 2011). Eine rezidivierende depressive Störung ist gekennzeichnet durch wiederholte depressive Episoden, wobei keine unabhängigen Episoden gehobener Stimmung und Überaktivität in der Vorgeschichte bestehen (Dilling et al., 2014).

Im Schnitt verlaufen etwa 35-85\% aller depressiven Erkrankungen rezidivierend (Farb, Anderson, Bloch \& Segal, 2011; Hardeveld, Spijker, Graaf, Nolen \& Beekman, 2010, 2013). 
Den Ergebnissen von Kessler et al. (2009) zufolge variiert die Anzahl der Episoden und deren Dauer in den verschiedenen Geburtskohorten. Jüngere Geburtskohorten sind häufig von mehreren, dafür kürzeren Episoden betroffen, ältere Geburtskohorten erleben hingegen längere Episoden und eher chronische Verläufe (Uhmann \& Wittchen, 2010). Der Großteil der befragten Erkrankten berichtet dabei eine Episodendauer von mehr als 21 Wochen. Nach Hautzinger (2015b) weisen etwa ein Viertel aller depressiven Erkrankungen einen chronischen Verlauf auf, d.h. die Dauer einer depressiven Episode überschreitet zwei Jahre. Abbildung 1 zeigt den typischen Verlauf der verschiedenen Formen affektiver Erkrankungen über den Lebensverlauf.

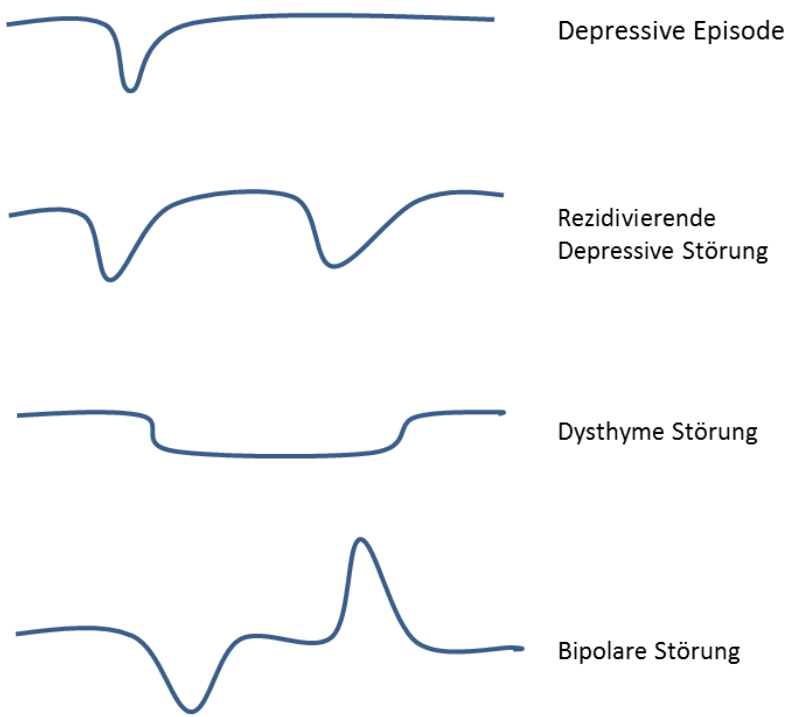

Abbildung 1. Typischer Verlauf affektiver Störungen über den Lebensverlauf (Depressive Episode Ausschlag nach unten, manische Episode Ausschlag nach oben).

Auch das heterogene Erscheinungsbild der Depression im Verlauf macht die Früherkennung zu einer Herausforderung. Die Interaktion der Entstehungsbedingungen, welche in der Berechnung eines Erkrankungsrisikos zu berücksichtigen ist, stellt sich für die Erstmanifestation einer Depression anders dar als im Rahmen eines Rezidivs. Anzunehmen ist die Notwendigkeit einer unterschiedlichen Gewichtung von Risiko- und Schutzfaktoren für eine depressive Erstmanifestation und ein Rezidiv. 


\section{5. Ätiologische Modelle}

Die Ätiologie depressiver Erkrankungen ist ein komplexer und vielschichtiger Prozess. Zahlreiche in Wechselwirkung stehende Umwelt-, Risiko- und Schutzfaktoren spielen in interpersonell individueller Konstellation eine Rolle. Entstehung und Aufrechterhaltung der Depression werden in daraus abgeleiteten biopsychosozialen Störungsmodellen abgebildet (Jacobi \& Kessler-Scheil, 2013). Beteiligt an der Entstehung einer Depression sind mitunter genetische, neuromodulatorische, biochemische, endokrine, entwicklungspsychologische, lern-und lebensgeschichtliche, kulturelle, interaktionale, ökonomische, organische, kognitive und psychophysiologische Komponenten, die über distale und proximale Ebenen in Wechselwirkung stehen und den Gehirnstoffwechsel beeinflussen (Hautzinger, 2015a). Nach Hautzinger (2013) lassen sich die Ursachen einer akuten Depression aufgrund der Komplexität der Einfluss-, Risiko- und Entwicklungsfaktoren auf individueller Ebene auch in der Einzelfallbetrachtung kaum zweifelsfrei klären und empirisch nur schwer prüfen. Aufgrund der heterogenen Erscheinungsformen und der vielschichtigen Entstehungsbedingungen der Depression gibt es bislang keine einheitliche Störungstheorie (Beesdo-Baum \& Wittchen, 2011). Hautzinger (2013) schlägt ein Modell vor, nach dem bestimmte distale Risikofaktoren die Grundlage der Entwicklung einer Depression bilden. Unter distalen Risikofaktoren werden allgemeine psychosoziale Rahmenbedingungen verstanden, die mit einem erhöhten Erkrankungsrisiko assoziiert sind und nicht unmittelbar, sondern über vermittelnde Prozesse zu einer Beeinträchtigung führen, zum Beispiel weibliches Geschlecht und niedriger Sozialstatus (Hautzinger, 2013; Tröster, 2009). Diese können wiederum zu affektiven, kognitiven, neurobiologischen und interaktionellen Vulnerabilitäten führen. Er geht davon aus, dass diese Vulnerabilitäten in Kombination mit bestimmten Stressoren intensiviert werden und infolge dessen das Erkrankungsrisiko steigt. Die dadurch entstandenen „Narben“ (Hautzinger, 2013, S. 39) werden im Lebensverlauf durch weitere Belastungen reaktiviert. Eine darüber erfolgte Intensivierung wird als proximales Risiko verstanden. Proximale Risikofaktoren stehen demnach in einer direkten Beziehung zur Beeinträchtigung, darunter zum Beispiel Temperament, Informationsverarbeitungsstil, sozialer Rückzug oder Mangel an sozialen Verstärkern. Jede depressive Episode führt daraufhin zu einer weiteren Verstärkung der Vulnerabilität. Die von 
Hautzinger (2013) zusammengestellten Risikofaktoren sind zur Übersicht in Tabelle 2 dargestellt.

Tabelle 2

Distale und proximale Risikofaktoren in der Entstehung einer Depression nach Hautzinger (2013)

\section{Risikofaktoren Beispiele}

\section{Prädisponierende Faktoren - distale Risikofaktoren}

Demographische Faktoren Weibliches Geschlecht, jüngeres Lebensalter, niedriger Sozialstatus, beengte finanzielle Verhältnisse, Arbeitslosigkeit, Hausfrauenrolle, Familienstand

Vorerkrankungen

Biologische Faktoren

Persönlichkeitsfaktoren

Sozialisationsbedingungen

\section{feir}

Subklinische Depression, Schilddrüsenanomalie, Virusinfektion, Immunschwäche, Angst, Persönlichkeitsstörung

Heredität der Depression, Heredität für Angst/Neurotizismus, Anfälligkeit und Veränderbarkeit der Neuromodulatoren, fetaler Hypercortisolismus, Mikroglia-Aktivierung und entzündung

Introversion,

abhängig-dependente

Persönlichkeit, Rückversicherungstendenz

Depressive Modelle, mangelnde Reagibilität/Vernachlässigung, feindselige/intrusive Überstimulation, fehlende vertrauensvolle Bezugsperson, fehlende soziale Unterstützung, frühe Traumatisierung

\section{Psychobiologische Vulnerabilität}

Biologisch

Erhöhte Erregbarkeit von Teilen des limbischen Systems, Anfälligkeit für Dysregulation neuromodulatorischer Achsen, Sensibilität bestimmer ZNS-Strukturen und Zellverbände, Änderung der Rezeptorstruktur der Messenger-Mechanismen, gestörte Schlaf-Wach-Regulation, Immunkompetenzdefizite 


\begin{tabular}{|c|c|}
\hline \multirow[t]{3}{*}{ Kognitiv-emotional } & Löschungsresistenz, schnelle \\
\hline & Auslösbarkeit \\
\hline & Selbstaufmerksamkeit, negative Schemata \\
\hline \multirow[t]{3}{*}{ Sozial-interaktiv } & \multirow{2}{*}{$\begin{array}{l}\text { Negative Selbst- und Fremdpräsentation, Vermeidungstendenz } \\
\text { sozialer Interaktionen, eingeschränkte sozial-kommunikative }\end{array}$} \\
\hline & \\
\hline & $\begin{array}{l}\text { Fertigkeiten, reduzierte Repertoire an Bewältigungsstrategien } \\
\text { und Problemlöseverhalten }\end{array}$ \\
\hline
\end{tabular}

\section{Reaktionsmuster - proximale Faktoren}

Biologisch

Funktionales Defizit im Locus Coeruleus, TransmitterImbalance, CRF-Überreaktion ${ }^{1}$, starker Cortisolanstieg, HHNAchsen-Dysfunktion, Zunahme an Beta-Rezeptoren und Cytokinen, Störung der Schlafarchitektur und der circadianen Rythmen

Kognitiv

Pessimistischer Explanationsstil, Persistenz negativer Kognitionen (Rumination), selektive negative Informationsverarbeitung, unspezifische Zugriffe auf autobiographische Erinnerungen, reduzierter Zugriff auf positive Inhalte

Sozial-interaktiv Sozialer Rückzug, soziale Anklammerung, reduzierte soziale Verstärker, mangelnde Initiative bzw. Fertigkeiten den Verstärkermangel zu kompensieren

${ }^{1}$ Corticotropin releasing factor ${ }^{2}$ Hypothalamus-Hypophysen-Nebennierenrinden-Achse

Dieses in Klinik und Forschung allgemein verwendete und bislang gültige Erklärungsmodell zur Entstehung der Depression wird als Vulnerabilitäts-Stress-Modell bezeichnet. Vereinfacht dargestellt lässt sich nach dem Vulnerabilitäts-Stress-Modell der Ausbruch einer depressiven Erkrankung über das Zusammenwirken bestimmter Vulnerabilitätskonstellationen mit kritischen Lebensereignissen und deren Kombination mit weiteren proximalen Belastungsfaktoren erklären (Beesdo-Baum \& Wittchen, 2011). Bislang konnten lediglich Teilkomponenten und einzelne Entwicklungspfade des oben beschriebenen Modells empirisch bestätigt werden (Beesdo-Baum \& Wittchen, 2011; 
Hautzinger, 2013). Kendler, Prescott, Myers und Neale (2003) konnten in zwei umfangreichen Zwillingsstudien mit Hilfe von Strukturgleichungsmodellen signifikante Entwicklungspfade einer akuten Depression darstellen. Für folgende Risikofaktoren fand sich in den genannten Untersuchungen bei retrospektiven Befragungen ein statistisch signifikanter Zusammenhang mit der Entwicklung einer Depression: Genetisches Risiko, Mangel an elterlicher Wärme, Missbrauch in der Kindheit, Verlust eines Elternteils, Neurotizismus, geringer Selbstwert, früher Beginn von Ängsten, sozial auffälliges Verhalten, Bildungsdefizite und Schulschwierigkeiten, traumatische Erfahrungen in der Lebenszeit, fehlende soziale Unterstützung, Substanzmissbrauch, Scheidung/Trennung, Vorgeschichte einer Depression, Eheprobleme im letzten Jahr, Schwierigkeiten im letzten Jahr sowie kritische Lebensereignisse im letzten Jahr. Die gefundenen Zusammenhänge lassen sich aufgrund des retrospektiven Studiendesigns und der methodischen Konzeption der Studie zwar nicht kausal interpretieren, liefern jedoch wichtige Hinweise auf mögliche Interaktionen einzelner Risikofaktoren und bilden eine wichtige theoretische Grundlage für weitere Untersuchungen in der Ätiologieforschung.

Bemerkenswert ist, dass bei den etablierten Entstehungsmodellen depressiver Erkrankungen in der Prädiktionsforschung zwei wesentliche Aspekte bisher wenig Beachtung finden. Zum einen sind dies protektive oder Schutzfaktoren und zum anderen Veränderungen auf psychopathologischer Ebene. Beide Aspekte sind, wie bereits erwähnt, auch in den Forschungsbestrebungen zur Früherkennung und Risikokalkulation der Depression bisher kaum berücksichtigt. Insbesondere bezüglich protektiver Faktoren gibt es jedoch zahlreiche empirische Befunde, welche deren Einfluss auf das Erkrankungsrisiko belegen (Cairns, Yap, Pilkington \& Jorm, 2014; Masten, Cutuli, Herbers \& Reed, 2009; Rutter, 2000). Die Risikokalkulation könnte sich durch den Einbezug von Schutzfaktoren gänzlich verändern. So resultieren nach Albee (1980) Fehlentwicklungen im Bereich der Entwicklungspsychologie aus einem ungünstigen Verhältnis von Risikofaktoren zu Schutzfaktoren. Diese Betrachtungsweise ließe sich analog auf die Entstehung depressiver Erkrankungen beziehen und für die Früherkennung nutzen. Unter Risikofaktoren werden dabei Vulnerabilität und Stressoren zusammengefasst, unter Schutzfaktoren Resilienz und Ressourcen. Die Entstehung einer Depression ist demnach auch bei Vorliegen einer ausgeprägten Vulnerabilität und dem Erleben von Stress nicht die notwendige Folge, sofern vor Ausbruch der Erkrankung adäquate Anpassungsstrategien und Resilienz vorliegen. Gut 
untersuchte Schutzfaktoren in Bezug auf psychische Erkrankungen sind beispielsweise hohe Selbstwirksamkeit, positives Familienklima und soziale Unterstützung (Klasen et al., 2015). Für alle drei Faktoren konnte ein positiver Zusammenhang mit psychischer Gesundheit nachgewiesen werden (Klasen et al., 2015).

Auch psychopathologische Aspekte in Form depressiver Prodromalsymptome sind in den gängigen Erklärungsmodellen der Prädiktion wenig integriert, obwohl auch hier die Ergebnisse empirischer Untersuchungen deren Berücksichtigung zur Kalkulation eines Erkrankungsrisikos nahe legen (Pine, Cohen \& Brook, 2001; Syed Sheriff et al., 2015; Woodward \& Fergusson, 2001). Anzunehmen ist demnach eine noch weitaus komplexere Gestaltung der Ätiologie depressiver Störungen als durch bisherige Modelle abgebildet. Eine valide Früherkennung kann nur gelingen, wenn zumindest eine Annäherung an ein umfassendes Prädiktionsmodell erfolgt.

In der vorliegenden Arbeit sollen daher neben den bekannten und empirisch gut untersuchten Risikofaktoren auch psychopathologische Aspekte und protektive Mechanismen in die Modellbildung der Früherkennung und Rezidivvorhersage einbezogen werden. Es wird von einem komplexen Zusammenspiel von Risiko- und Schutzfaktoren ausgegangen. Eine Integration von Schutzfaktoren und psychopathologischen Veränderungen scheint essentiell, da sich unter der Einwirkung dieser Faktoren mit hoher Wahrscheinlichkeit andere und zusätzliche Wechselwirkungen und Algorithmen ergeben als unter der alleinigen Berücksichtigung der genannten Risikofaktoren. Das ätiologische Modell als auch die methodische Herangehensweise zur Untersuchung dieser Zusammenhänge müssen demnach erweitert werden. Die methodische Umsetzung der Überprüfung dieser Einflüsse stellt eine große Herausforderung dar, vor allem da die Wechselwirkungen einzelner Komponenten eine Vielzahl an möglichen Entwicklungspfaden generieren. Kraemer, Stice, Kazdin, Offord und Kupfer (2001) haben diesbezüglich fünf verschiedene klinisch bedeutsame Pfade der Risikofaktoreninteraktion konzeptualisiert und operationalisiert: Proximale, überlappende und unabhängige Risikofaktoren sowie Mediatoren und Moderatoren. Alle kausalen Faktoren bilden den Autoren zufolge demnach Risikofaktoren, nicht alle Risikofaktoren sind jedoch kausal. Die Ätiologie der Depression ergibt sich damit interindividuell aus einer Vielzahl an möglichen Interaktionen von 
Risikofaktoren. Die Risikoprofilerstellung wird letztlich auf individualisierter Ebene am besten realisiert werden können.

Nachfolgend werden die bisherigen Forschungsergebnisse zu einzelnen aus der Literatur bekannten Risiko- und Schutzfaktoren übersichtsartig dargestellt und diskutiert. Der Schwerpunkt liegt dabei insbesondere auf den soziodemographsichen Risikofaktoren sowie auf Persönlichkeitsfaktoren, Vorerkrankungen und Sozialisationsbedingungen, unter Ausschluss neurobiologischer, endokriner, physikalischer und immunologischer Aspekte. Diese Aspekte sollen in einer weiteren Studie mit dem hier konzeptualisierten Entwicklungsmodell depressiver Erkrankungen kombiniert und in ein umfassendes Früherkennungsmodell integriert werden.

\subsection{Risikofaktoren}

Zahlreiche empirische Studien aus den vergangenen Jahren liefern Ergebnisse zu den Risikofaktoren in der Entstehung depressiver Erkrankungen (Cairns, Yap, Pilkington \& Jorm, 2014; Hidaka, 2013; Sullivan, Neale \& Kendler, 2000). Eine Vielzahl dieser Untersuchungen bezieht sich jedoch nur auf einzelne, isolierte Entwicklungspfade und Risikofaktoren. Übersichtsarbeiten oder Studien, welche ein multifaktorielles Ätiologiemodell berücksichtigen, finden sich in der aktuellen Literatur nur wenige (Cairns et al., 2014; Kendler, Gardner \& Prescott, 2002; Kendler, Prescott, Myers \& Neale, 2003). Dabei wird die Bedeutung einer Kombination der einzelnen Risikofaktoren zu einem integrierten Entstehungsmodell wie oben beschrieben bereits seit Langem diskutiert (siehe Akiskal \& McKinney, 1975; Kendler, Gardner \& Prescott, 2002). Im Folgenden sollen die wichtigsten Ergebnisse im Hinblick auf die Risikokalkulation depressiver Erkrankungen zusammengefasst werden. Eine Schwierigkeit ist dabei die Unterscheidung zwischen den Entstehungsbedingungen der Erstmanifestation und den Risikofaktoren eines Rezidivs depressiver Erkrankungen. In der Literatur werden dazu differenzielle Faktoren diskutiert (Burcusa \& lacono, 2007). 


\subsubsection{Vulnerabilität}

Unter Vulnerabilität wird eine bestimmte Veranlagung oder erhöhte Anfälligkeit verstanden, unter Risikobedingungen mit psychopathologischen Symptomen zu reagieren (Schmidt \& Göppel, 2008). Nach Beesdo-Baum und Wittchen (2011) bilden prädisponierende konstitutionelle Faktoren genetischer bzw. familiengeschichtlicher Art sowie frühe adverse soziale und umweltbedingte Ereignisse eine erhöhte Vulnerabilität für depressive Erkrankungen. Die Autoren nennen diesbezüglich eine positive Familiengeschichte für Depressionen, frühkindliche Traumata, Persönlichkeit und Temperament, kritische Lebensereignisse, chronische Belastungen sowie soziale und psychologische Faktoren. Als spezifische soziodemographische Vulnerabilitätsfaktoren gelten Alter, Geschlecht, sozioökonomischer Status und Familienstand (Burcusa \& lacono, 2007). Ein Zusammenhang zwischen den genannten soziodemographischen Variablen und einem erhöhten Depressionsrisiko zeigt sich in fast allen epidemiologischen Studien (Kessler \& Bromet, 2013). Demnach lassen sich schon aus der Erfassung spezifischer soziodemographischer Daten und der Familienanamnese erste Informationen zur Abschätzung des Erkrankungsrisikos erheben.

\subsubsection{Alter}

Das Alter klärt einen Teil der Varianz in den Prävalenzraten der Depression auf (Akhtar-Danesh \& Landeen, 2007). Die Lebenszeitprävalenzraten sind in der Altersgruppe der 35-49-Jährigen für Frauen und auch für Männer am höchsten (Kessler et al., 2010). Bezüglich der 12-Monats-Prävalenzen zeigt sich ein ähnliches Bild in der Altersverteilung. Die Altersgruppe der 35-49-Jährigen bildet nach Uhmann und Wittchen (2010) damit die Hochrisikogruppe für depressive Erkrankungen und auch die Gruppe, welche am häufigsten in stationärem und ambulantem Behandlungssetting zu finden ist. Bei Kindern unter zehn Jahren finden sich depressive Störungen hingegen sehr selten. In der Adoleszenz steigen die Erkrankungsraten (Beesdo et al., 2009; Buysse et al., 2007). Auffallend geringer sind auch die Prävalenzen in der Altersgruppe der über 65-Jährigen. In älteren Geburtskohorten ist zudem eine geringere 12-Monats-Prävalenz feststellbar (Kessler et al., 2010). Diskutiert werden ungeeignete diagnostische Instrumente, Geburtskohorteneffekte, verzerrte 
Erinnerungsleistungen im höheren Lebensalter, eine verstärkte Konfundierung mit körperlichen Erkrankungen sowie höhere Resilienz im Alter, welche die niedrigeren Erkrankungsraten bedingen könnten (Beesdo-Baum \& Wittchen, 2011; Uhmann \& Wittchen, 2010). Das aktuelle Lebensalter ist unter Berücksichtigung der Prävalenzraten in der Bevölkerung eine zentrale und aussagekräftige Komponente und sollte daher sowohl bezüglich einer Erstmanifestation als auch eines Rezidivs in die Einschätzung des Erkrankungsrisikos einbezogen werden. Das aktuelle Lebensalter sollte daher als gewichtete Komponente in ein mathematisches Modell zur Risikoberechnung integriert werden.

\subsubsection{Geschlecht}

Das Geschlecht bildet neben dem Alter einen der bedeutsamsten epidemiologischen Risikofaktoren für die Entstehung einer depressiven Erkrankung. Es ist der am häufigsten bestätigte Risikomarker für Depressionen (Kessler, 2003; Uhmann \& Wittchen, 2010). Ab der Adoleszenz finden sich deutliche genderspezifische Unterschiede in den Prävalenzzahlen (Uhmann \& Wittchen, 2010). Nach den Ergebnissen epidemiologischer Studien sind Frauen fast doppelt so häufig von Depressionen betroffen wie Männer (Jacobi et al., 2004). Wie bereits dargestellt, liegt die durchschnittliche Lebenszeitprävalenz für das weibliche Geschlecht deutlich höher als für das männliche (Uhmann \& Wittchen, 2010). Zur Erklärung der unterschiedlichen Erkrankungsraten bei Männern und Frauen existieren zahlreiche Hypothesen. Diskutiert werden unter anderem hormonelle Unterschiede, Persönlichkeitsfaktoren, soziale und Umweltfaktoren sowie kritische Lebensereignisse, so genannte Life Events (Beesdo-Baum \& Wittchen, 2011). Die Ergebnislage empirischer Untersuchungen zu den genderspezifischen Unterschieden in der Entstehung und Häufigkeit der Depression ist bislang nicht eindeutig und die Integration in ätiologische Modelle noch unzureichend (Piccinelli, 2000). Neueste Forschungsergebnisse klassifizieren allerdings genderspezifische Entstehungsfaktoren der Erstmanifestation als auch des Rezidivs depressiver Erkrankungen (Kendler \& Gardner, 2014; Oquendo et al., 2013). Demzufolge spielen Persönlichkeitsfaktoren und zwischenmenschliche Probleme in der Ätiologie der Depression bei Frauen eine stärkere Rollen als bei Männern, während sich bei Männern häufiger finanzielle oder arbeitsbedingte Probleme, Gesetzeskonflikte und sexueller Missbrauch in der Kindheit in der Entstehung der Depression finden. Bezüglich des 
Rezidivrisikos fanden Oquendo et al. (2013) ein hohes Rückfallrisiko gleichermaßen für Männer und Frauen bei Vorliegen von erlebtem sexuellen Missbrauch in der Vergangenheit, eines hohen Bildungsstands und einem früheren Ersterkrankungsalter. Der Stand der Forschung lässt darauf schließen, dass das weibliche Geschlecht ein spezifischer Risikofaktor für die Erstmanifestation einer Depression ist, jedoch nicht für einen rezidivierenden Verlauf (siehe Burcusa \& lacono, 2007). Wie bereits erwähnt steht das Geschlecht als Risikofaktor depressiver Erkrankungen jedoch in der Kritik. Der Literatur zufolge erfassen die gängigen Klassifikationssysteme eher weibliche als männliche Attribute der Depression (Martin et al., 2013), was den Autoren zufolge zu einer ungleichen Verteilung der Prävalenzraten führt. Eine zukünftige Depressionsforschung sollte demnach sowohl unterschiedliche genderspezifische Vulnerabilitätsfaktoren als auch spezifische Symptomkonstellationen für Männer und Frauen berücksichtigen. Für die Früherkennung und Berechnung des Erkrankungsrisikos ist der Faktor Geschlecht den dargestellten Ergebnissen zufolge von zentraler Bedeutung. Es wird jedoch zu prüfen sein, ob sich die Erkrankungswahrscheinlichkeiten bei Berücksichtigung genderspezifischer Entstehungsmechanismen und Symptomkonstellationen angleichen.

\subsubsection{Psychiatrische Erkrankungen in der Familie}

Die Forschung der letzten 20 Jahre postuliert eine positive Familiengeschichte als einen validen Risikofaktor für die häufigsten chronischen Erkrankungen (Wilde et al., 2014). In den Bereichen der kardiovaskulären Erkrankungen, Krebserkrankungen und Diabetes ist die Familiengeschichte als Instrument zur Einschätzung des Erkrankungsrisikos sowie zur Ableitung von Interventionen und Präventionsmaßnahmen bereits etabliert (Valdez, Yoon, Qureshi, Green \& Khoury, 2010). Die Familiengeschichte stellt dabei ein äußerst ökonomisches und effizientes Instrument zur Kalkulation des Erkrankungsrisikos dar. Eine Abbildung sowohl genetischer als auch nicht-genetischer Entstehungsfaktoren ist dabei ohne kostenintensive Genanalysen möglich. Gemeinsame Genkomponenten, geteilte Umwelteinflüsse und behaviorale Aspekte formen ähnliche Risikoprofile innerhalb einer Familie, was für die Nutzbarkeit und Operationalisierung der Familiengeschichte als prädiktives Instrument in der Prävention chronischer Erkrankungen spricht (Yoon et al., 2002). Obwohl zahlreiche Familien-, Zwillings- und Adoptionsstudien eine starke familiäre 
Häufung auch bei affektiven Erkrankungen zeigen (Kern, Sheldrick, Schmidt \& Minkwitz, 2012; Merikangas et al., 2002; Sullivan, Neale \& Kendler, 2000; Valdez, Yoon, Qureshi, Green \& Khoury, 2010), ist die Heranziehung einer psychiatrischen Familienanamnese zur Risikokalkulation vor allem mit Bezug zu depressiven Erkrankungen noch wenig genutzt. Empirische Studien konnten ein erhöhtes Erkrankungsrisiko für Kinder depressiver Eltern sowie eine Assoziation einer positiven psychiatrischen Familiengeschichte mit einem rezidivierenden Krankheitsverlauf nachweisen (Lieb, Isensee, Höfler, Pfister \& Wittchen, 2002; Weissman et al., 2016). Weissmann und Kollegen (2016) konnten in einer Follow-Up Studie mit einem Erfassungszeitraum von 30 Jahren bestätigen, dass Kinder depressiver Eltern ein dreifach erhöhtes Erkrankungsrisiko im Vergleich zu Nachkommen von gesunden Probanden hatten. Die Daten zeigen weiterhin ein leicht erhöhtes Risiko für einen präpubertären Beginn der Depression in der Gruppe der Nachkommen depressiver Eltern. Die multifaktorielle Genese der Depression spricht für die Relevanz und unbedingte Berücksichtigung der Familiengeschichte in der Risikokalkulation, da hier sowohl genetische als auch nicht-genetische Entstehungsfaktoren abgebildet werden können. Kritisch zu bemerken bleibt hier eine relativ unspezifische Ergebnislage aus den Studien zur Familiengeschichte (Kessler, 2003). Neben depressiven Erkrankungen waren häufig andere psychiatrische Erkrankungen, insbesondere Angst oder Alkoholabhängigkeit Prädiktoren für depressive Störungen bei den Nachkommen (Merikangas, Risch \& Weissman, 1994). Die Integration einer allgemeinen psychiatrischen Familiengeschichte in die Modellbildung zur Früherkennung der Depression wird deshalb als sinnvoll erachtet.

Eine genetische Vulnerabilität scheint nachweislich zudem bei Early Onset Depression (EOD) eine stärkere Rolle zu spielen als bei Late Onset Depression (LOD) (Baron, Mendlewicz \& Klotz, 1981). Die Befunde zur Relevanz einer positiven Familiengeschichte depressiver Erkrankungen bei EOD sind dahingehend relativ konsistent (Korten, Comijs, Lamers \& Penninx, 2012; Tozzi et al., 2008). Eine familiäre Häufung affektiver Erkrankungen wird zusätzlich spezifisch mit einem chronischen Verlauf der Depression in Zusammenhang gebracht (Hölzel, Härter, Reese \& Kriston, 2011).

Insgesamt weisen die Studienergebnisse zur Familiengeschichte darauf hin, dass sowohl genetische als auch nicht-genetische Faktoren einen Einfluss auf die Entstehung affektiver Erkrankungen haben. Die Berücksichtigung einer Interaktion zwischen genetischen Risikofaktoren und Umwelteinflüssen ist für die Erstellung eines Risikoprofils eine zentrale 
Bedingung. Die Erhebung der Familienanamnese ist dahingehend ein ökonomisches Verfahren. Dies wird von Allgemein- und Hausärzten dennoch häufig nicht realisiert. Gründe dafür sind nach Yoon, Scheuner, Jorgensen und Khoury (2009) der erhebliche Zeitaufwand zur Erfassung dieser Informationen, die Frage der Abrechnungsmodalität sowie Unsicherheiten in der Interpretation der Ergebnisse und daraus abgeleitete adäquate Beratung der identifizierten Hochrisiko-Personen. Im Rahmen der Früherkennung scheint die Erhebung dieser Komponenten jedoch unumgänglich. Eine adäquate Ableitung des Erkrankungsrisikos und entsprechender Präventionsmaßnahmen kann jedoch nur unter Berücksichtigung zahlreicher anderer Einflussfaktoren, wie der hier genannten, gelingen.

\subsubsection{Familienstand}

Neben alters- und genderspezifischen Effekten zeigen sich hohe Prävalenzraten depressiver Störungen besonders bei geschiedenen, getrennt lebenden oder alleinstehenden Personen (Andrade et al., 2003; Kessler \& Bromet, 2013; Weissman, 1996). Die Depressionsraten sind für Getrennte, Geschiedene und Verwitwete zum Teil höher als für Unverheiratete. Es konnten zudem spezifische Interaktionen des Familienstandes mit dem Geschlecht nachgewiesen werden, wonach verheiratete Frauen in Studien höhere Depressionsraten aufwiesen als alleinstehende Frauen und der Zusammenhang zwischen Scheidung und Depression bei Männern stärker ausgeprägt war als bei Frauen (BeesdoBaum \& Wittchen, 2011; Paykel, Brugha \& Fryers, 2005; Weissman, 1996). Der Familienstand wird insgesamt eher als ein Risikofaktor für die Entstehung der Depression im Sinne der Erstmanifestation und weniger für ein Rezidiv diskutiert (siehe Burcusa \& lacono, 2007).

Insgesamt lassen die Studienergebnisse darauf schließen, dass soziodemographische Variablen wie Geschlecht, Familienstand und sozioökonomischer Status spezifische Risikofaktoren für die Entstehung der Depression im Rahmen einer Erstmanifestation darstellen (Burcusa \& lacono, 2007). 


\subsubsection{Ersterkrankungsalter}

Ein niedriges Ersterkrankungsalter wird in zahlreichen Studien als Risikofaktor für einen rezidivierenden Verlauf der Depression sowie für stärkere Beeinträchtigungen, einen ausgeprägten Schweregrad und eine längere Dauer der Symptomatik diskutiert (Hardeveld, Spijker, Graaf, Nolen \& Beekman, 2013; Korten, Comijs, Lamers \& Penninx, 2012; Pettit, Lewinsohn, Roberts, Seeley \& Monteith, 2009; Rohde, Lewinsohn, Klein, Seeley \& Gau, 2013; Wilson, Vaidyanathan, Miller, McGue \& lacono, 2014). Das mittlere Ersterkrankungsalter für Depressionen liegt den Ergebnissen großer epidemiologischer Studien zufolge bei etwa 26 Jahren (Kessler et al., 2009). Für das Ersterkrankungsalter der Depression ist laut Kessler et al. (2009) allerdings ein Interkohorten-Effekt zu beobachten. Das niedrigste durchschnittliche Ersterkrankungsalter wird mit knapp 18 Jahren für die jüngste Geburtskohorte der 18-34Jährigen berichtet. In höheren Altersgruppen ist ein deutlich höheres Ersterkrankungsalter zu beobachten (siehe Tabelle 3). Bei Integration des Ersterkrankungsalters in die Rezidivvorhersage müssen daher Kohorteneffekte berücksichtigt werden.

Tabelle 3

Mittelwerte des Ersterkrankungsalters in den verschiedenen Geburtskohorten nach Kessler et al. (2009)

\section{Geburtskohorte (Alter in Jahren)}

18-34

$50-64$

$+65$

\section{$M$ (in Jahren)}

17.8

25.5

33.1

43.0

In der Literatur findet sich weiterhin die Unterscheidung zwischen EOD und LOD, der sogenannten „Altersdepression“, die eine Erstmanifestation der Depression im höheren Lebensalter bezeichnet (Heser et al., 2013). Es handelt sich hierbei um eine dichotome Kategorisierung des Ersterkrankungsalters. Eine Differenzierung zwischen EOD und LOD ist aufgrund angenommener Unterschiede in den Entstehungsbedingungen und Risikofaktoren der Erkrankung wichtig, jedoch existieren bislang keine einheitlich definierten Kategorien. In der Regel wird eine Erstmanifestation der Depression ab einem Alter von 50 Jahren als LOD 
klassifiziert (Notzon, Alferink \& Arolt, 2016). Die diagnostischen Kriterien beider Phänotypen unterscheiden sich nicht, wohingegen neueste Studien unterschiedliche Risikofaktoren, Symptome und Krankheitsverläufe für EOD und LOD postulieren (Korten, Comijs, Lamers \& Penninx, 2012; Wilson, Vaidyanathan, Miller, McGue \& lacono, 2014). Eine Studie von Tozzi et al. (2008) zeigte zum Beispiel einen signifikanten Zusammenhang zwischen einem frühen Ersterkrankungsalter und einer positiven Familiengeschichte für Depression. Unterschiedliche ätiopathogenetische Modelle werden dahingehend diskutiert. Die Ergebnislage ist jedoch insgesamt inkonsistent. So konnten zum Beispiel Grayson und Thomas (2013) in einer systematischen Übersichtsarbeit keine maßgeblichen Unterschiede in den klinischen Charakteristika von LOD und EOD feststellen. Auf psychopathologischer Ebene lassen sich lediglich vermehrt kognitive Beeinträchtigungen in Form von Gedächtnisund Konzentrationsstörungen bei der LOD finden, welche jedoch nicht zwingend mit der Depression assoziiert sind (Naismith, Norrie, Mowszowski \& Hickie, 2012). Wichtig ist hier aber auch die Abgrenzung zu dementiellen Erkrankungen und eine Berücksichtigung anderer hirnorganischer Einflussfaktoren, wie zum Beispiel des Apoplex, der bei etwa 30\% der Betroffenen eine Depression nach sich zieht („post stroke depression“; Schottke \& Giabbiconi, 2015). Auch Patienten mit einer Demenz weisen eine erhöhte Wahrscheinlichkeit auf an einer Depression zu erkranken, wobei die depressiven Symptome häufig schon vor der Manifestation kognitiver Beeinträchtigungen nachweisbar sind (Notzon et al., 2016). Beachtenswert ist zudem die Häufung somatischer Beschwerden und körperlicher Erkrankungen im höheren Lebensalter, welche depressionsähnliche Symptome hervorrufen können, wie zum Beispiel Schlafstörungen oder körperliche Schwäche (Notzon et al., 2016). Bei der Einschätzung des Erkrankungsrisikos spielen für die LOD demzufolge andere Faktoren eine Rolle als für die EOD. Auch bestimmte Medikamente wie zum Beispiel beta-Blocker, welche im höheren Lebensalter häufiger eingenommen werden, können depressionsähnliche Symptome erzeugen (Avorn, Everitt \& Weiss, 1986; Thiessen, Wallace, Blackburn, Wilson \& Bergman, 1990). Die Berücksichtigung von körperlichen Erkrankungen und regelmäßiger Medikamenteneinnahme ist für die Diagnostik und Therapie der Depression im höheren Lebensalter zentral (Notzon et al., 2016) und sollte daher auch bei der Kalkulation eines Erkrankungsrisikos im Rahmen der Früherkennung für Personen in höherem Lebensalter berücksichtigt werden. Die Forschungsbefunde sprechen vor allem für ein erhöhtes Erkrankungsrisiko im höheren Lebensalter bei Vorliegen einer körperlichen 
Erkrankung mit Beeinträchtigung der Funktionsfähigkeit, nach körperlichen Eingriffen und bei Einnahme depressiogener Medikamente (Antihypertensiva, Hormone, Antiparkinsonmittel und Kortikosteroide; Hautzinger, 1997). Anzunehmen ist, dass das Risiko an einer Depression zu erkranken im höheren Lebensalter somit anderen Einflussfaktoren unterliegt als in der Jugend oder dem jungen Erwachsenenalter.

Aus den dargestellten Befunden kann die Notwendigkeit der Erhebung und Berücksichtigung soziodemographischer und anamnestischer Variablen, wie Alter, Geschlecht und Ersterkrankungsalter im Rahmen der Früherkennung und Risikokalkulation der Depression geschlussfolgert werden. Das aktuelle Lebensalter und das Geschlecht bilden zentrale Einflussgrößen in der Abschätzung des Erkrankungsrisikos, insbesondere bezüglich der Erstmanifestation einer Depression. Das Ersterkrankungsalter liefert darüber hinaus wichtige Informationen über ein mögliches Rezidivrisiko. Die Unterscheidung zwischen LOD und EOD ist in der Konzeptbildung insofern relevant und nutzbar, als dass die Risikoberechnung einer Erstmanifestation im höheren Lebensalter vielfach andere Faktoren berücksichtigen muss als bei jüngeren Menschen.

\subsubsection{Depressive Episode in der Vorgeschichte}

Die Schätzungen für rezidivierende Verläufe depressiver Erkrankungen liegen wie bereits erwähnt zwischen 35 und 85\% (Curry et al., 2011; Eaton et al., 2008; Mueller et al., 1999; wie zitiert in Pettit, Hartley, Lewinsohn, Seeley \& Klein, 2013). Depressive Episoden in der Vorgeschichte bilden einen spezifischen Risikofaktor für erneute Erkrankungen und Chronifizierung (Pettit et al., 2013). In epidemiologischen Untersuchungen konnten die Personen mit dem höchsten Risiko an einer Depression zu erkranken anhand der Anzahl früherer Episoden identifiziert werden (Bulloch, Williams, Lavorato \& Patten, 2014). Die Anzahl der früheren Episoden konnte in einer neueren Metaanalyse sogar als einer der besten Prädiktoren für einen Rückfall extrahiert werden (Hardeveld et al., 2010). Es existieren verschiedene Theorien zum Rezidivrisiko, darunter die sogenannte "Scar-Theorie" (Rohde, Lewinsohn \& Seeley, 1990). Diese Theorie postuliert eine spezifische Vulnerabilität für Depression bei Personen mit einer depressiven Epsiode in der Vorgeschichte. Die Depression an sich führt demnach zu langfristigen physiologischen und kognitiven Veränderungen, welche das Rückfallrisiko erhöhen. Dies bedeutet, dass schon eine 
einmalige depressive Episode das Risiko für eine erneute Erkrankung zu einem späteren Zeitpunkt erhöht (Burcusa \& lacono, 2007). Nach Hautzinger (2013) verstärkt jede depressive Episode die vorliegende Vulnerabilität. Daneben gibt es zahlreiche Theorien und empirische Befunde, die strukturelle und funktionelle Veränderungen im Gehirn durch die Depression postulieren, welche wiederum für ein erhöhtes Rückfallrisiko verantwortlich gemacht werden (Hamilton et al., 2012; Savitz \& Drevets, 2009; Whitfield-Gabrieli \& Ford, 2012). Der Verlauf der Erkrankung und die Anzahl der bereits durchlaufenen Episoden eignen sich diesen Befunden zufolge zur Rezdivvorhersage und sollten in ein umfassendes Modell zur Risikoberechnung integriert werden.

\subsubsection{Persönlichkeitsfaktoren}

Persönlichkeit und Temperament werden seit der Antike mit psychischer Gesundheit in Verbindung gebracht und beschreiben bedeutsame Konstrukte in der Erforschung der Ätiologie psychischer Störungen (Kotov et al., 2010). Es gibt dahingehend zahlreiche Forschungsbefunde, welche insbesondere eine Assoziation zwischen Neurotizismus und psychischen Störungen belegen (Ormel et al., 2013). Der Zusammenhang ist nach den Ergebnissen einer Metaanalyse von Kotov et al. (2010) für Depression und Angststörungen mit einer Effektstärke von 2.0 (Cohen`s $d$ ) am höchsten. Den Autoren zufolge sind derart hohe Effektstärken in der Ätiologieforschung sehr selten, woraus sich die unbedingte Berücksichtigung in der Früherkennungsforschung ergibt. Zahlreiche Studien bestätigen einen Zusammenhang zwischen Neurotizismus und Depression, sowohl für die Entstehung der Depression als auch für einen rezdivierenden Verlauf (Burcusa \& lacono, 2007; Kendler, Gatz, Gardner \& Pederse, 2006; Noteboom, Beekman, Vogelzangs \& Penninx, 2016). Die Studienlage ist insgesamt bislang jedoch noch inkonsistent (Hardeveld et al., 2010). Zur Erklärung der Assoziation von Neurotizismus und der Entstehung psychischer Erkrankungen gibt es nach Ormel et al. (2013) fünf verschiedene theoretische Modelle. Die VulnerabilitätsHypothese setzt Neurotizismus mit Prozessen in Verbindung, welche den Weg für die Entwicklung einer psychischen Störung bahnen. Demzufolge werden psychische Erkrankungen entweder direkt durch eine hohe Ausprägung an Neurotizismus verursacht oder die Anfälligkeit für psychische Störungen steigt unter Einfluss von Stressoren bei gleichzeitigem Vorliegen hoher Neurotizismusausprägungen. Die Spektrum-Hypothese geht 
davon aus, dass Neurotizismus und psychische Erkrankungen unterschiedliche Manifestationen des zugrunde liegenden Prozesses darstellen. Danach wären hohe Neurotizismuswerte in ihrer Erscheinung äquivalent mit den Symptomen einer psychischen Erkrankung. Die Common-Cause-Hypothese postuliert gemeinsame Ursachen beider Erscheinungen und sieht Neurotizismus als Risikofaktor für psychische Erkrankungen, aufgrund gemeinsamer genetischer und umweltbedingter Einflüsse. Das Scar-Modell beschreibt eine dauerhafte Veränderung der Persönlichkeit durch den Einfluss einer psychischen Erkrankung. Die Veränderungen sind dem Modell zufolge auch nach Remission der Erkrankung persistierend. Das State-Modell geht dagegen von temporären Veränderungen in Richtung Neurotizismus im Rahmen einer psychischen Erkrankung aus, welche jedoch mit Remission der Erkrankung wieder auf ihr Ursprungsniveau zurückkehren. In einer aktuellen Übersichtsarbeit kommen Ormel et al. (2013) zu dem Ergebnis, dass Neurotizismus ein Risikofaktor für den Ausbruch einer psychischen Störung ist und den Zusammenhang zwischen negativen Lebensereignissen und psychischen Erkrankungen verstärkend moderiert. Allerdings gehen die Autoren davon aus, dass keines der genannten Modelle den Zusammenhang zwischen Neurotizismus und psychischer Erkrankung vollständig erfassen kann und Neurotizismus eher als unspezifischer Marker für ein generelles Erkrankungsrisiko zu werten ist. Zukünftige Forschung muss nach Ormel et al. (2013) zudem untersuchen, ob Interventionen zur Reduktion von Neurotizismus die Entstehung einer psychischen Erkrankung oder den Behandlungserfolg positiv beeinflussen. Die Autoren gehen von einem Mediatoreffekt, im Sinne eines Behandlungserfolgs durch die Reduktion von Neurotizismuswerten aus. Diese Ergebnisse sprechen insgesamt für eine hohe Relevanz von Persönlichkeitsfaktoren, insbesondere von Neurotizismus im Rahmen der Ätiologieforschung und damit auch Prävention der Depression. Zu untersuchen gilt es weiter, in welcher Form diese spezifische Persönlichkeitsvariable auf die Entwicklung einer depressiven Erkrankung wirkt und ob sie eher im Sinne der Vulnerabilitäts-Theorie einen allgemeinen generellen Risikofaktor für eine Ersterkrankung darstellt, oder auch das Rezidivrisiko im Sinne der Scar-Theorie erhöht. Erwähnenswert ist weiterhin die Assoziation der Depression mit Extraversion. Konkret fand sich in empirischen Untersuchungen neben dem Zusammenhang zwischen Depression und hohen Neurotizismusausprägungen auch ein Zusammenhang zwischen Depression und einer niedrigen Ausprägung von Extraversion (Clark, Watson \& Mineka, 1994; Malouff, Thorsteinsson \& Schutte, 2005). Auf Basis 
empirischer Befunde in einer Bevölkerungsstichprobe, die dies bestätigten, schlussfolgern Grav, Stordal, Romild und Hellzen (2012) die Notwendigkeit der Identifikation von Personen mit hohen Neurotizismus- und niedrigen Extraversionsausprägungen zur Prävention der Depression.

\subsubsection{Frühe Traumata}

Ein weiterer gut untersuchter und in retrospektiven Studien empirisch bestätigter Risikofaktor für Depressionen sind frühe Traumata und adverse Entwicklungsbedingungen in der Kindheit. In der Literatur finden sich viele Hinweise darauf, dass frühkindliche Traumata mit Beginn, Verlauf, Schweregrad und dem komorbiden Charakter depressiver Erkrankungen assoziiert sind (z.B. Bernet \& Stein, 1999). Unter frühen traumatischen Erlebnissen werden unter anderem Verlust der Eltern, Scheidung der Eltern, psychiatrische Auffälligkeiten bei primären Bezugspersonen, schwere Krankheit in der Kindheit, Gewalterfahrungen in der Familie, Deprivation, Vernachlässigung und Mangel an Unterstützung verstanden (Carr, Martins, Stingel, Lemgruber \& Juruena, 2013). Zusammengefasst werden die genannten Kindheitstraumata in die Kategorien emotionaler Missbrauch, sexueller Missbrauch, körperlicher Missbrauch, körperliche Vernachlässigung und emotionale Vernachlässigung (Carr et al., 2013). Eine starke, häufige oder lang anhaltende Aktivierung der körperlichen Stressreaktion in der Kindheit führt nach Lindert et al. (2014) bei fehlender Unterstützung primärer Bezugspersonen zu manifesten und überdauernden physiologischen Veränderungen, wodurch das Risiko für spätere psychische Erkrankungen steigt (Lindert et al., 2014). Einer Metaanalyse von Lindert et al. (2014) zufolge finden sich in der Mehrzahl der empirischen Untersuchungen erhöhte Wahrscheinlichkeiten für Depressionen und Angsterkrankungen bei Personen mit frühkindlichen Traumata. Die Chancen sind im Schnitt um das zweifache erhöht. Eine Metaanalyse von Mandelli, Petrelli und Serretti (2015) untersuchte spezifisch den Zusammenhang zwischen frühkindlichen Traumata und Depressionen. Auch hier zeigte sich ein erhöhtes Risiko bei Erfahrung früher Traumata im späteren Lebensverlauf an einer Depression zu erkranken. Allerdings ließ sich hier eine spezifische Rolle für emotionalen Missbrauch und Vernachlässigung bezüglich depressiver Erkrankungen nachweisen. Den Autoren zufolge bilden sexueller und körperlicher Missbrauch eher unspezifische Risikofaktoren für psychische Störungen. Für den frühen 
Verlust eines Elternteils und Scheidung der Eltern konnten in der genannten Metaanalyse keine signifikanten Assoziationen mit einem erhöhten Erkrankungsrisiko für Depression nachgewiesen werden. Kendler et al. (2002) wiesen weiterhin in einer empirischen Untersuchung an weiblichen Zwillingen in einem Strukturgleichungsmodell einen spezifischen Entwicklungspfad der Depression über adverse frühkindliche Erfahrungen nach. Die Ergebnisse empirischer Untersuchungen befürworten zudem die Hypothese frühkindlicher Traumata als spezifischen Risikofaktor für einen chronischen Verlauf der Depression (Wiersma et al., 2009). Die Erfassung frühkindlicher adverser Erlebnisse ist auf Basis der dargestellten Befunde als ein wichtiger Bestandteil des Ätiologiemodells und auch eines Früherkennungsinventars zur Kalkulation des Erkrankungsrisikos der Depression zu werten.

\subsubsection{Stressoren: Kritische Lebensereignisse, Arbeits- und Alltagsbelastungen}

Stressoren werden in zahlreichen Forschungsbefunden mit der Entstehung der Depression in Verbindung gebracht (Kendler, Karkowski \& Prescott, 1999). Der Begriff Stressor umfasst unter anderem psychosoziale Belastungsfaktoren und Lebensereignisse (Life Events), wie zum Beispiel den Tod einer nahestehenden Person, Trennung, Scheidung und andere Verlustereignisse (Beesdo-Baum \& Wittchen, 2011). Nach Kaluza (2015) werden dabei im Allgemeinen kritische Lebensereignisse, Arbeits- und Alltagsbelastungen unterschieden. Als Arbeitsbelastungen werden hier Bedingungen beschrieben, die bei längerem Andauern zu Erschöpfung führen können, wie zum Beispiel Arbeitsüberlastung, mangelnde Kontrolle, unzureichende Belohnung, Fehlen von Fairness und widersprüchliche Werte sowie eine Imbalance zwischen Anforderungen und Entscheidungsspielraum. Unter Alltagsbelastungen werden die tägliche Erfüllung von Aufgaben, psychosoziale Konflikte und Spannungen, Rumination bezüglich der täglichen Anforderungen, Überflutung mit Informationen und Flexibilitätsdruck verstanden. Ein Übermaß an Belastungen oder das vermehrte Auftreten unangenehmer Ereignisse kann in der klinischen Praxis zumeist als ein auslösender Faktor einer depressiven Erkrankung nachvollzogen werden. Dabei wird unterschieden in situative, aktuelle und dauerhafte Belastungen oder Lebensereignisse (Hautzinger, 2013). Die Wahrnehmung einer Belastung ist jedoch nicht ausschließlich durch die situativen Gegebenheiten definiert, sondern hängt vielmehr von den subjektiven und 
individuellen Bewältigungsmechanismen, der persönlichen Bedeutungszuschreibung sowie den Kompetenz- und Kontrollgefühlen einer Person ab (Kaluza, 2015), was wiederum für eine Wechselwirkung mit spezifischen Schutzfaktoren und Copingmechanismen spricht. Besonders das Auftreten kritischer Lebensereignisse wird neben einer positiven Familiengeschichte für affektive Erkrankungen und früheren depressiven Episoden als einer der konsistentesten und am besten untersuchten Prädiktoren für Depressionen berichtet (Monroe, Slavich \& Gotlib, 2014). Kendler und Gardner (2014) fanden in Zwillingsstudien zu den Risikofaktoren der Depression insbesondere bei Männern eine positive Assoziation zwischen spezifischen Stressoren und der Entstehung depressiver Erkrankungen, darunter die stärksten Effekte für finanzielle Probleme, Probleme in Verbindung mit der Arbeit und Probleme mit dem Gesetz. Für Frauen zeigten sich dagegen stärkere Zusammenhänge mit Beziehungskonflikten und schweren Erkrankungen im nahen sozialen Umfeld. Beachtung fanden in den letzten Jahren insbesondere auch Forschungsbefunde $\mathrm{zu}$ den stressassoziierten biologischen Mechanismen und Risikofaktoren depressiver Erkrankungen. Eine Dysregulation der HHN-Achse (Hypothalamus-Hypophysen-Nebennierenachse) und die Wechselwirkung mit der HHN-Stressantwort auf immunologische Prozesse werden in neuesten Untersuchungen in Verbindung mit der Ätiologie der Depression gebracht (siehe Hammen, 2015). Zusammenfassend lässt sich sagen, dass im Rahmen zahlreicher empirischer Untersuchungen Stresserleben in Form kritischer Lebensereignisse, Arbeitsoder Alltagsbelastung als relevanter Faktor in der Ätiologie der Depression identifiziert wird. Auch strukturelle und funktionelle Veränderungen neuronaler Prozesse sowie genetische Faktoren werden in der Wechselwirkung mit Stress mit depressiven Erkrankungen in Verbindung gebracht (Hammen, 2015). Die Erfassung kritischer Lebensereignisse sowie die Erfassung von Arbeits- und Alltagsbelastung sollten daher in einem Früherkennungsinventar enthalten sein. Auch biologische, neuromodulatorische, immunologische und hormonelle Aspekte, die in einem Zusammenhang mit Stressbelastung stehen, sollen im Rahmen des vorliegenden Forschungsvorhabens in Zukunft integriert werden. 


\subsubsection{Schutzfaktoren und Copingmechanismen}

Unter protektiven Faktoren werden Merkmale verstanden, die bei Vorliegen von Risikofaktoren im Sinne von Vulnerabilitäten und psychosozialen Belastungen vor der Entwicklung einer psychischen Störung schützen und diesem entgegenwirken (Blanz, 2006). Protektive Faktoren oder auch Schutzfaktoren sind im Allgemeinen seltener untersucht als Risikofaktoren depressiver Störungen. In der Literatur finden sich dazu im Vergleich weniger Studien (z.B. Klasen et al., 2015; Masten, Cutuli, Herbers \& Reed, 2009; Rutter, 2000; Snyder \& Lopez, 2009). Die Datenlage bezüglich protektiver Faktoren ist vor allem im Bereich der Erwachsenen dünn. Die bisherige Forschung konzentrierte sich zumeist auf Kinder und Jugendliche und auf die Frage, welche Faktoren den Ausbruch einer Depression verhindern oder die Symptome abmildern können. Wie bereits erwähnt, gibt es nach Klasen et al. (2015) im Bereich der Kinder- und Jugendforschung drei Schutzfaktoren, welche empirisch bisher gut untersucht und bestätigt sind: Hohe Selbstwirksamkeit, soziale Unterstützung und ein positives Familienklima. Klasen et al. (2015) konnten beispielsweise in einer prospektiven Studie zeigen, dass die depressive Symptomatik bei Jugendlichen mit einem Anstieg an Ressourcen sank. Bei Jugendlichen, welche bestimmte Risikofaktoren (z.B. psychische Erkrankung bei einem Elternteil) aufwiesen, wurde die Symptombildung durch protektive Faktoren, wie zum Beispiel ein positives Familienklima und vorhandene soziale Unterstützung moderiert. Einen bedeutsamen Einfluss konnten die Autoren hier insbesondere für Selbstwirksamkeit zeigen, welche sich bei den untersuchten Jugendlich maßgeblich auf die Symptombildung auswirkte. Hohe Selbstwirksamkeit ging dabei mit einer geringeren Symptomausprägung einher. Bei Anstieg der berichteten Selbstwirksamkeit war zudem eine Reduktion depressiver Symptome im Verlauf beobachtbar. Weiterhin kommen Santini, Koyanagi, Tyrovolas, Mason und Haro (2015) in einem aktuellen Review zu dem Ergebnis, dass die wahrgenommene soziale Unterstützung signifikant protektive Effekte bezüglich depressiver Symptome aufweist. Besonders emotionale und instrumentelle Unterstützung ebenso wie ein breites und differentielles soziales Netz haben demzufolge einen positiven Einfluss auf die Depression. Während eine geringe wahrgenommene soziale Unterstützung mit der Entwicklung einer Depression assoziiert ist.

Insgesamt ist von einer moderierenden Wirkung protektiver Faktoren in der Ätiologie der Depression auszugehen. Die Erfassung insbesondere von Selbstwirksamkeit, sozialer 
Unterstützung und des Familienklimas sollte Bestand eines Früherkennungsinventars und der Berechnung des Erkrankungsrisikos sein.

\subsubsection{Prodromalphase und Prodromalsymptome}

Kommt es zu einer Wechselwirkung einschlägiger Risikofaktoren kann sich eine depressive Erkrankung entwickeln. Zumeist geschieht dies schleichend. Dieser Zeitraum wird als Prodrom der Erkrankung bezeichnet. Die Prodromalphase einer Erkrankung beschreibt den Zeitraum vor dem Ausbruch einer Erkrankung, in dem schon frühe Symptome oder Veränderungen auftreten, welche sich jedoch vom klinischen Erscheinungsbild der Erkrankung unterscheiden (Fava \& Tossani, 2007). Im Allgemeinen wird davon ausgegangen, dass das Prodrom nur retrospektiv definiert werden kann (Eaton, Badawi \& Melton, 1995; Kovacs \& Lopez-Duran, 2010). Nach Fava und Kellner (1991) beginnt die Prodromalphase mit dem Auftreten erster Krankheitsanzeichen und reicht bis zur charakteristischen Manifestation der voll ausgeprägten Symptome einer Erkrankung. Die Prodromalsymptome stehen mit der Erkrankung vermutlich über physiologische Pfade in Verbindung und gehen typischerweise mit einer Beeinträchtigung der Funktion einher (Kovacs \& Lopez-Duran, 2010). Unter dem Begriff Prodrom wird dahingehend nicht nur eine Veränderung auf psychopathologischer Ebene verstanden, sondern auch eine Phase zentraler Vulnerabilitäten und bestimmter funktionaler Beeinträchtigungen (Kovacs \& Lopez-Duran, 2010). Forschung zur Prodromalphase psychischer Erkrankungen existiert bisher insbesondere im Bereich der Psychosen und der bipolaren Störung (Syed Sheriff et al., 2015; Woodberry, Shapiro, Bryant \& Seidman, 2016; Yung et al., 2012). Auf dem Gebiet der Psychosen ist die Prodromalforschung bereits gut etabliert und es konnten neurokognitive, neuroanatomische, funktionale, chemische und psychopathologische Veränderung vor Beginn der voll ausgeprägten Erkrankung nachgewiesen werden (siehe Addington et al., 2015; z.B. Koutsouleris et al., 2015). Zum Prodrom der Depression existiert vergleichsweise wenig empirische Forschung (siehe Jackson, Cavanagh, \& Scott, 2003).

Die Prodromalsymptome der Depression werden je nach Studie entweder als mildere Form der Symptome einer Depression oder als qualitativ verschiedene Symptome zur Depression beschrieben, ein Konsens diesbezüglich besteht bislang nicht (Fava \& Kellner, 1991; Jackson, Cavanagh \& Scott, 2003; Kovacs \& Lopez-Duran, 2010; Syed Sheriff et al., 
2015). Die Dauer der Prodromalphase im Rahmen depressiver Erkrankungen variiert empirischen Untersuchungen zufolge zwischen zwei Tagen und 23 Monaten (lacoviello, Alloy, Abramson \& Choi, 2010; Jackson, Cavanagh \& Scott, 2003). Frühere Studien hypothetisieren die intraindividuelle Konsistenz der Prodromalsymptome über mehrere depressive Episoden hinweg (Fava et al., 1990) ähnlich der intraindividuellen Konsistenz depressiver Symptome in Vollausprägung innerhalb der Rezidive (Paykel, Prusoff \& Tanner, 1976). In den Studien zu Prodromalsymptomen der Depression wurden vor allem Angst, Anspannung, Reizbarkeit, Verlust von Interesse, Schlafschwierigkeiten, Antriebsminderung, emotionale Taubheit, gedrückte Stimmung, gastrointestinale Probleme, Appetitverlust, Konzentrationsstörungen und Mangel an Energie berichtet (siehe Jackson et al., 2003). lacoviello et al. (2010) fanden in einer Längsschnittuntersuchung zu den Prodromalsymptomen bei depressiven Episoden sieben zentrale Frühsymptome bei Personen die eine Depression entwickelten im Vergleich zu einer Kontrollgruppe, welche nach Alter, Geschlecht, Herkunft, Bildungsstand und Risikofaktoren angepasst war. Hier wurden gedrückte Stimmung, Interessen- oder Freudverlust, Konzentrationsschwierigkeiten, Gefühle der Hoffnungslosigkeit, Sorgen, reduzierter Selbstwert und Reizbarkeit berichtet. Auffallend scheint eine teilweise Deckung mit den Kernsymptomen der Depression wie Antriebsminderung, gedrückte Stimmung und Freud- oder Interessenverlust, jedoch auch das Vorkommen von differenziellen Symptomen wie zum Beispiel Reizbarkeit. Die Ergebnisse einer aktuellen qualitativen Untersuchung zur Prodromalsymptomatik (Syed Sheriff et al., 2015) bestätigen dieses Bild. Die Untersuchungen zeigen, dass die Symptome der Prodromalphase in weiten Teilen mit den diagnostischen Kriterien der Depression übereinstimmen, die frühesten Veränderungen hingegen von diesen verschieden sind. Berichtet werden hier Traurigkeit, Reizbarkeit, Schuldgefühle und Schlafstörungen. Zu den frühesten Veränderungen gehören nach Syed Sheriff et al. (2015) Unsicherheit bezüglich der Identität, sozialer Rückzug und eine Beeinträchtigung der Rollenfunktion. Bei einem Großteil der Probanden fanden sich auch Symptome von Angst. Diese Befunde lassen darauf schließen, dass die psychopathologischen Veränderungen im Rahmen eines depressiven Prodroms, wie oben genannt, entweder eine mildere Stufe der Kernsymptome einer depressiven Episode darstellen oder aber auch differenzielle Veränderungen umfassen. Nach Hetrick et al. (2008) lassen sich aus den bisherigen Forschungsergebnissen drei zentrale Schlussfolgerungen zum Prodrom der Depression ziehen: Erstens scheinen Ängstlichkeit und 
Reizbarkeit zu den Kernsymptomen des Prodroms zu gehören. Zweitens bildet eine subsyndromale leichte Form der Depression einen Risikofaktor für die Entwicklung einer depressiven Episode, was für die Entstehung einer Depression auf einem Kontinuum spricht, und drittens sind die gleichen Prodromalsymptome in der Erstmanifestation mit hoher Wahrscheinlichkeit auch in nachfolgenden Rezidiven vorhanden.

Ein interessanter Aspekt ist weiterhin die in der Forschung beschriebene Deckung von Prodromal- und Residualsymptomen sowie der inverse zeitliche Verlauf dieser psychopathologischen Veränderungen (Fava, Grandi, Zielezny, Canestrari \& Morphy, 1994; Mahnert, Reicher, Zalaudek \& Zapotoczky, 1997). Demzufolge beschreiben die zuerst auftretenden Symptome auch diejenigen Symptome, die als letzte bis zur Remission präsent sind. Die Psychopathologie der Prodromal- und Residualphase ist nach lacoviello et al. (2010) ein geeigneter Faktor zur Vorhersage des weiteren Verlaufs der Erkrankungsphase und zur Einschätzung einer generellen zukünftigen Prognose. Die Identifikation und Klassifizierung von subsyndromalen und prodromalen Zuständen bildet damit eine bedeutsame Voraussetzung für die Prävention der Depression analog zur Psychoseforschung (Hetrick et al., 2008; Kovacs \& Lopez-Duran, 2010). Die Prodromalphase stellt ein kritisches Zeitfenster für Prävention dar, zumal Interventionen in einem frühen Krankheitsstadium bessere Behandlungsergebnisse und eine generell günstigere Prognose versprechen als interventionelle Maßnahmen in einem späteren Stadium der Erkrankung (Hetrick et al., 2008).

\subsubsection{Dauer der unbehandelten Episode}

Als ein weiterer Risikofaktor für einen rezidivierenden Krankheitsverlauf und eine höhere Anzahl an depressiven Rezidiven gilt die Dauer der unbehandelten depressiven Erkrankung (duration of untreated illness, DUI) oder die Dauer der unbehandelten Episode (duration of untreated episode, DUE). Inhaltlich definiert ist dieses Konzept als Zeitraum zwischen dem Beginn der Erkrankung und der ersten angemessenen Behandlung dieser Erkrankung (Dell'Osso \& Altamura, 2010). Differenziert wird hier zwischen der Dauer der unbehandelten Erkrankung bei Erstmanifestation (DUI) und der Dauer einer unbehandelten Episode bei rezidivierendem Krankheitsverlauf (DUE). Die DUI wurde bisher vor allem in der Psychoseforschung untersucht, im Bereich der affektiven Störungen gibt es dazu jedoch 
noch realtiv wenige Befunde (Ghio et al., 2015). Der Schwerpunkt der bisherigen Untersuchungen zur DUI liegt auf der Assoziation der DUI und dem Behandlungserfolg bei depressiven Störungen. Einer Metaanalyse von Ghio et al. (2015) zufolge ist ein längeres Interval, in welchem keine Behandlung der Erkrankung erfolgt, ein Prädiktor für ein schlechteres Ansprechen der Patienten auf antidepressive Medikation, für geringere Remissionsraten, ein höheres Risiko der Chronifizierung und eine höhere Anzahl an Rezidiven. Methodisch problematisch ist in einer Vielzahl der Studien die unsaubere Differenzierung der Konzepte DUI und DUE. Nach Ghio et al. (2015) sind dies zwei essentiell verschiedene Konstrukte. Gemäß der Kindling-Hypothese (Kendler, Thornton \& Gardner, 2000) wird das Risiko eines Rezidivs mit wachsender Anzahl an Episoden, aufgrund neurobiologischer Veränderungen immer unabhängiger von äußeren Einflüssen und ein autonomer Prozess. Dieser Zusammenhang wird nach Ghio et al. (2015) noch verstärkt, wenn die Erkrankung unbehandelt bleibt. Aus diesen Ergebnissen lässt sich ableiten, dass die Erfassung sowohl von DUI als auch von DUE eine Aussagekraft im Rahmen der Risikokalkulation depressiver Rezdive haben kann. Damit muss der Zeitraum zwischen dem Beginn der Erkrankung und der ersten angemessenen Behandlung als kritisches Zeitfenster für frühe Interventionen betrachtet werden, um den Schweregrad der Erkrankung abzumildern und den Verlauf der Erkrankung günstig zu beeinflussen.

\subsubsection{Wechselwirkungen der Risiko- und Schutzfaktoren}

Obwohl die genannten Risiko- und Schutzfaktoren in weiten Teilen gut untersucht und empirisch eine Assoziation mit der Entstehung und dem Verlauf depressiver Störungen nachgewiesen werden konnte, ist über die Wechselwirkung der einzelnen Faktoren relativ wenig bekannt. Die Beantwortung der Frage, warum beispielsweise bestimmte Personen nach kritischen Lebensereignissen an einer Depression erkranken, andere Personen jedoch nicht, liegt in der Interaktion von Risiko- und Schutzfaktoren. Entsprechende ätiologische Modelle müssen daher Mediator- und Moderatoreffekte berücksichtigen, um ein vollständiges Erklärungsmodell abzubilden.

In einer empirischen Untersuchung konnten Monroe et al. (2014) beispielsweise einen reziproken Zusammenhang zwischen Life Events und einer positiven Familiengeschichte für Depression zeigen. Probanden, die ein kritisches Life Event vor Ausbruch der depressiven 
Erkrankung berichteten, hatten eine fast vierfach geringere Wahrscheinlichkeit depressiver Erkrankungen in der Primärfamilie. Die Autoren schlussfolgern einen kausalen Zusammenhang zwischen kritischen Lebensereignissen und Depressionen sowie einen differentiellen Einfluss von Life Events auf die Entstehung einer depressiven Erkrankung unabhängig von einer positiven Familiengeschichte für Depressionen. Daraus ließe sich schlussfolgern, dass kritische Lebensereignisse einen unabhängigen und gewichtigen Faktor in der Ätiologie der Depression darstellen. Andere Ergebnisse finden sich für die Zusammenhänge zwischen Stress, depressiven Symptomen, depressiven Episoden in der Vorgeschichte und Persönlichkeitseigenschaften. Hammen et al. (2015) gehen zum Beispiel davon aus, dass die Assoziation zwischen Stress und Depression bidirektional und dynamisch ist und von den genannten Faktoren in vielfacher Weise beeinflusst wird, indem diese wiederum zu vermehrtem Auftreten kritischer Lebensereignisse und Stresserleben beitragen. Ebenso werden Wechselwirkungen zwischen Neurotizismus, Stresserleben und Depression (Barlow, Ellard, Sauer-Zavala, Bullis \& Carl, 2014) sowie dysfunktionaler kognitiver Informationsverarbeitung und Depression vermutet (Nolen-Hoeksema, 2000).

Auch für den Risikofaktor frühkindlicher Traumata lässt sich die Frage stellen, warum Personen mit traumatischen Erfahrungen in der Kindheit im späteren Leben an einer Depression erkranken und andere Personen trotz Vorliegen einer frühkindlichen Traumatisierung nicht. Die Wechselwirkung zwischen frühkindlichen Traumata und kritischen Lebensereignissen ist hier gut untersucht. Es gibt empirische Evidenz für eine erhöhte Sensitivität bei früh traumatisierten Personen für die negativen Folgen stressreicher Lebensereignisse (Kendler, Kuhn \& Prescott, 2004). Postuliert wird dahingehend eine Interaktion zwischen biologischen und Umweltbedingungen. Aber auch hier gilt es Copingfaktoren, Anpassungsleitung und Resilienz zu berücksichtigen (Mandelli et al., 2015). 


\section{Konzeptualisierung der Studie}

Wie aus den dargestellten Befunden ersichtlich, fordert die Komplexität der Ätiopathogenese der Depression ein dynamisches, alters- und genderspezifisches Modell, das vielfache Entwicklungspfade beschreibt und verschiedenste Formen der Risikofaktorinteraktion sowie moderierende und mediierende Einflüsse integriert. Das erste zentrale Ziel des vorliegenden Forschungsvorhabens ist daher die Modellbildung zur Ätiologie und die Darstellung der Entwicklungspfade einer Depression. Da für die Identifizierung von Personen, die ein hohes Erkrankungsrisiko für Depression aufweisen, bisher weder standardisierte Diagnoseinstrumente noch ausreichend Präventivprogramme existieren, besteht ein zweites zentrales Ziel dieses Projektes in der Entwicklung eines ökonomischen und prognostisch validen klinischen Fragebogens zur Früherkennung der Depression „DEEP-I“ (Depression Early Prediction - Inventory), welches sich aus dem genannten Ätiologiemodell ableitet. Mit Hilfe des Fragebogens sollen dann spezifische Risikoprofile generiert und ein prognostisch valider Risikoalgorithmus etabliert werden. Darauf aufbauend ist ein weiteres zukünftiges Ziel des Projekts die Konzeption und Evaluation individualisierter Präventionsmaßnahmen auf der Grundlage der abgeleiteten Hochrisikoprofile. Damit wird eine spezifische Passung von Hochrisikopersonen mit Präventivleistungen möglich. Der Einsatz psychopharmakologischer Medikamente oder langwährender psychotherapeutischer Maßnahmen kann so reduziert oder bestenfalls verhindert werden. Ziel der vorliegenden Arbeit ist die Darstellung der Konzeptualisierung des gesamten Forschungsvorhabens sowie die Auswertung und Diskussion erster Ergebnisse einer Pilotstudie. Besonderes Augenmerk gilt dabei spezifischen Risikofaktoren des depressiven Rezidivs sowie einer Analyse der klinischen Charakteristika der Prodromalphase.

Auf Grundlage des theoretischen Hintergrunds und der dargestellten Forschungsergebnisse lässt sich ein hypothetisches Bedingungsmodell der Depression konzeptualisieren. Die empirische Befundlage spricht für eine vierfaktorielle Struktur. Das apriori-spezifizierte Modell beinhaltet die nachfolgend dargestellten vier Faktoren. 

a) Vulnerabilitäten
b) Stressoren
c) Coping und Ressourcen
d) Psychopathologie im Sinne einer Prodromalsymptomatik

Die Studie lässt sich in drei Projektphasen einteilen, die aufeinander aufbauen und konsekutiv durchgeführt werden. Eine Übersicht über die Konzeptualisierung und die Studienplanung findet sich in Abbildung 3.

Die erste Phase des Projekts (Phase 1a) besteht in der Konzeptualisierung eines Modells zur Entstehung der Depression sowie in der Zusammenstellung eines geeigneten Messinstruments zur Erfassung der dargestellten Risiko- und Schutzfaktoren sowie psychopathologischen Veränderungen in der Prodromalphase, welche in der Entstehung depressiver Erkrankungen diskutiert werden.

In einer nachfolgenden Pilotstudie (Phase 1b) wird die Durchführbarkeit und Anwendbarkeit des konzipierten Messinstruments DEEP-I überprüft. Die Durchführung einer Machbarkeitsstudie begründet sich aus dem großen Umfang des generierten Itempools und einer hohen Durchführungsdauer. Die Zumutbarkeit und Compliance der Studienteilnehmer ist dahingehend zu überprüfen. Eine Auswertung der Daten aus der Pilotstudie erlaubt zudem erste Erkenntnisse über Unterschiede in Risiko- und Schutzmechanismen sowie Prodromalsymptomen in einer Population depressiv Erkrankter. Die Ergebnisse der Pilotstudie werden in der vorliegenden Arbeit dargestellt.

Die Evaluation des konzipierten Messinstruments DEEP-I soll anschließend in einer retrospektiven Querschnitterhebung realisiert werden (Phase 1c). Ein Stichprobenumfang von $n=600$ Probanden wird dabei angestrebt. Die Evaluation und Auswertung der Fragebögen ermöglicht mithilfe statistischer Verfahren eine Modellspezifizierung der Entwicklungspfade depressiver Erkrankungen. Die hypothetischen Bedingungszusammenhänge latenter und manifester Variablen aus dem a-priorispezifizierten Strukturgleichungsmodell werden überprüft, um eine geeignete Skalenkondensation vorzunehmen und die Itemformulierungen zu optimieren. Nach Durchführung und Auswertung der retrospektiven Querschnittuntersuchung anhand von Strukturgleichungsmodellen erfolgt die Erstellung eines kondensierten 
Früherkennungsinventars zur spezifizierten Früherkennung des Hochrisikozustandes der Depression. Eine Reduktion der Itemzahlen ist dabei unbedingt anzustreben, da das DEEP-I in seiner ersten Fassung mit dem Anspruch der Erfassung möglichst vieler Einflussfaktoren sehr umfangreich und damit nicht ökonomisch einsetzbar ist. Ein vereinfachtes theoretisches Strukturgleichungsmodell ist in Abbildung 2 dargestellt. Das abgeleitete Modell kann die Grundlage einer individualisierten Risikoprofilerstellung bilden, interindividuell werden sich jedoch vermutlich unterschiedliche Wechselwirkungen und Gewichtungen der einzelnen Risikofaktoren ergeben. Das Modell erhebt in seiner ersten Fassung keinen Anspruch auf Vollständigkeit. Es ist davon auszugehen, dass die Modellbildung im Verlauf anhand weiterer Forschungsergebnisse ergänzt und verändert werden wird. Eine Überprüfung des Modells kann nur anhand entsprechender Fallzahlen erfolgen und ist Ziel späterer Studienphasen.

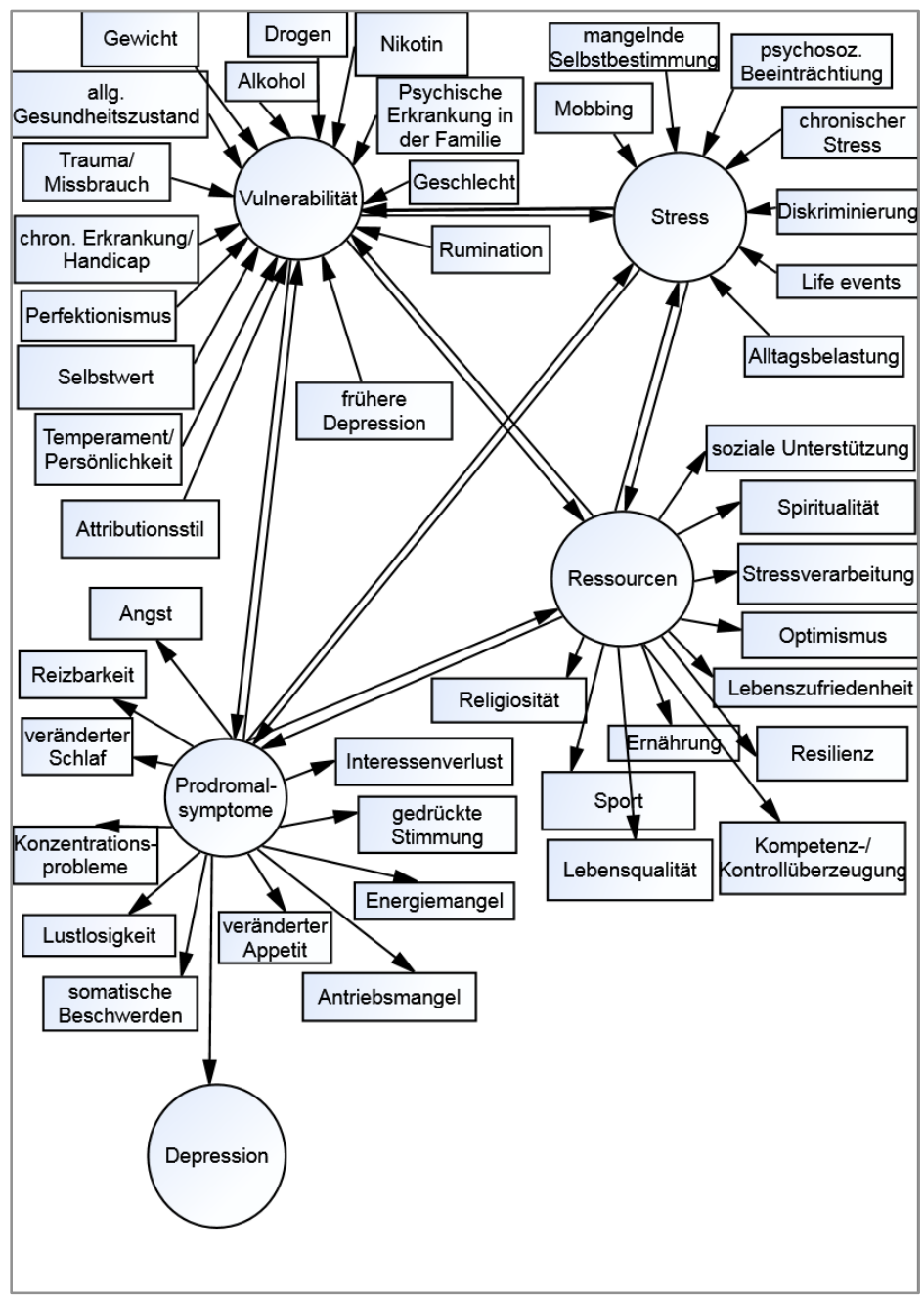

Abbildung 2. A-priori-spezifiziertes vereinfachtes Strukturgleichungsmodell für die hypothetischen Zusammenhänge der latenten und manifesten Variablen. Angenommene Wechselwirkungen sind durch Doppelpfeile dargestellt. Prüfung des Strukturgleichungsmodells in Phase 1c geplant. 
Das DEEP-I soll zur individualisierten Früherkennung der Depression eingesetzt werden und in einer sich anschließenden Longitudinaluntersuchung (Phase 2a) anhand einer risikoangereicherten Stichprobe evaluiert werden. Ziel ist die Entwicklung eines Prognosealgorithmus für depressive Erkrankungen. Dafür sollen „Hochrisiko-Personen“ hinsichtlich der Entwicklung einer depressiven Erkrankung über einen Zeitraum von zwei Jahren alle drei Monate mithilfe des „DEEP-I“ befragt werden. Aus diesen Daten soll der genannte Risikoalgorithmus generiert werden.

Eine Validierung des Algorithmus erfolgt im Anschluss in einer prospektiven Longitudinaluntersuchung (Phase 2b). Der erstellte Risikoalgorithmus soll auf prognostische Validität in der Normalbevölkerung geprüft werden. Ziel ist die Vorhersage des prozentualen Erkrankungsrisikos auf der Grundlage des Antwortverhaltens im DEEP-I.

Die adäquate und differenzierte Erfassung von Hochrisikozuständen ermöglicht dann den Weg für die Konzeption individualisierter und zielgruppenspezifischer Präventionsprogramme (Phase 3). Die abgeleiteten Risikoprofile sollen zur Generierung von geeigneten Interventionen herangezogen werden. Das Ziel ist dabei, das Ausbrechen der Erkrankung zu verhindern oder die Schwere des Verlaufs abzumildern.

\begin{tabular}{|c|c|}
\hline Ziel & Methode \\
\hline \multicolumn{2}{|c|}{ DEEP-I Phase 1a } \\
\hline $\begin{array}{l}\text { Entwicklung eines Inventars zur Früherkennung und Prädiktion } \\
\text { depressiver Erkrankungen. Generierung eines umfassenden } \\
\text { Itempools. }\end{array}$ & A priori Modellspezifizierung und Fragebogenkonstruktion \\
\hline \multicolumn{2}{|c|}{ DEEP-I Phase 1b } \\
\hline Überprüfung der Anwendbarkeit und Durchführbarkeit & Pilotstudie, retrospektive Querschnitterhebung \\
\hline \multicolumn{2}{|c|}{ DEEP-I Phase 1c } \\
\hline $\begin{array}{l}\text { Generierung eines ökonomischen Itempools und Kondensation der } \\
\text { Skalen }\end{array}$ & retrospektive Querschnitterhebung, Fragebogenevaluation \\
\hline \multicolumn{2}{|c|}{ DEEP-I Phase 2a } \\
\hline $\begin{array}{l}\text { Validierung des generierten Inventars zur Früherkennung } \\
\text { depressiver Erkrankungen. Klassifikation von Prodromalverläufen \& } \\
\text { Entwicklung eines Prognose-Algorithmus }\end{array}$ & Prospektive Längsschnitterhebung bei Hochrisiko-Probanden \\
\hline \multicolumn{2}{|c|}{ DEEP-I Phase $\mathbf{2 b}$} \\
\hline Überprüfung der prognostischen Validität des Algorithmus & Prospektive Längsschnitterhebung in der Normalbevölkerung \\
\hline \multicolumn{2}{|c|}{ DEEP-I Phase 3} \\
\hline $\begin{array}{l}\text { Etablierung spezifischer und individualisierter } \\
\text { Präventionsprogramme für identifizierte Hochrisiko-Probanden }\end{array}$ & Konzeption und Evaluation präventiver Maßnahmen \\
\hline
\end{tabular}

Abbildung 3. Studienkonzeptualisierung und Ziele des Forschungsvorhabens. 


\section{Fragestellungen}

Das Projekt befindet sich aktuell am Ende der Phase 1b. Die vorliegende Arbeit umfasst die Beschreibung der Phasen 1a und 1b sowie die Auswertung und Darstellung erster Ergebnisse zur Pilotstudie. Die Schwerpunkte der vorliegenden Analysen liegen vor allem in den Bereichen spezifischer Risikofaktoren für einen rezdivierenden Krankheitsverlauf sowie der Untersuchung prodromalphasentypischer Charakteristika. Alle Analysen erfolgen anhand der in Phase $1 \mathrm{~b}$ gewonnen Daten aus der Pilotstudie. Weitere Auswertungen zu den Daten der Pilotstudie sind geplant und sollen in nachfolgenden wissenschaftlichen Arbeiten dargestellt werden.

\subsection{Risikofaktoren für einen rezidivierenden Verlauf der Erkrankung}

Psychiatrische Erkrankungen in der Familie. Es wird ein Zusammenhang zwischen einer positiven Familiengeschichte für psychiatrische Erkrankungen und einem rezidivierenden Krankheitsverlauf angenommen. Eine Häufung von psychischen Erkrankungen in der Familie wird bei Probanden mit einem rezidivierenden Krankheitsverlauf im Vergleich zu Probanden mit nur einer depressiven Episode im Lebensverlauf vermutet. Auf Grundlage der dargestellten Forschungsergebnisse wird weiterhin angenommen, dass Probanden mit einer positiven Familiengeschichte für psychiatrische Erkrankungen ein früheres Ersterkrankungsalter aufweisen als Probanden, in deren Familien keine psychiatrischen Erkrankungen vorliegen.

Familienstand. Auf der Grundlage der dargestellten Forschungsergebnisse wird vermutet, dass der Familienstand keinen Einfluss auf den Krankheitsverlauf hat. Eine Häufung alleinstehender oder geschiedener Personen in einem Kollektiv depressiver Patienten wird angenommen.

Ersterkrankungsalter. Es wird eine Assoziation zwischen dem Ersterkrankungsalter und dem Verlauf der Depression vermutet. Es wird angenommen, dass sich bei Probanden mit rezidivierenden Krankheitsverläufen ein niedrigeres Ersterkrankungsalter findet als bei Probanden mit einer einzelnen depressiven Episode im Lebensverlauf und sich bei Probanden mit einem niedrigen Ersterkrankungsalter auch eine höhere Anzahl an berichteten Episoden im Lebensverlauf findet. 
Persönlichkeitsfaktoren. Es wird weiterhin eine Assoziation zwischen der Ausprägung der Persönlichkeitsvariablen Neurotizismus mit einem rezidivierenden Verlauf und der Anzahl der im Lebensverlauf berichteten depressiven Episoden angenommen. Es wird davon ausgegangen, dass Probanden mit rezidivierenden Krankheitsverläufen höhere Neurotizismuswerte aufweisen als Probanden mit nur einer depressiven Episode im Lebensverlauf und ein Zusammenhang zwischen der Neurotizismusausprägung und der Anzahl der berichteten Episoden über den Lebensverlauf festzustellen ist. Für die Persönlichkeitsausprägung Extraversion werden inverse Zusammenhänge vermutet. Angenommen wird, dass Probanden mit einem rezidivierenden Krankheitsverlauf niedrigere Ausprägungen an Extraversion aufweisen als Probanden mit einer einzelnen Episode im Lebensverlauf und sich bei Probanden mit hohen Extraversionswerten auch weniger Episoden im Lebensverlauf finden. Explorativ untersucht werden soll zudem, ob Neurotizismus und Extraversion in Zusammenhang mit dem Ersterkrankungsalter stehen.

Frühe Traumata. Des Weiteren wird angenommen, dass frühkindliche Traumata mit dem Krankheitsverlauf assoziiert sind. Es wird angenommen, dass Probanden mit rezidivierenden Krankheitsverläufen höhere Werte von emotionaler oder körperlicher Vernachlässigung, emotionalem, körperlichem und sexuellem Missbrauch aufweisen, als Probanden mit nur einer depressiven Episode im bisherigen Lebensverlauf.

\subsection{Charakteristika der Prodromalphase und Dauer der unbehandelten Episode}

Angenommen wird eine Prodromalphasendauer von mehreren Monaten. Es werden interindividuell hohe Streubreiten vermutet. Qualitätive Analysen sollen eine Beschreibung der berichteten Prodromalsymptome und deren Häufigkeiten beinhalten. Es wird eine teilweise Überlappung mit den diagnostischen Kritieren der Depression erwartet. In weiteren explorativen Analysen sollen die Dauer der Prodromalphase und die Dauer der unbehandelten Episode getrennt für Geschlecht, Altersgruppen und Krankheitsverlauf untersucht werden. 


\section{Methode}

\subsection{Stichprobe}

In der vorliegenden Studie wurden Personen mit diagnostizierten depressiven Erkrankungen untersucht. Befragt wurden Patienten des LMU Klinikums für Psychiatrie und Psychotherapie und der CIP-Tagesklinik in München. Die Abbildung 4 zeigt das CONSORT Diagramm zur Stichprobengewinnung und die entsprechenden Fallzahlen zur Pilotstudie. In die Studie eingeschlossen wurden Personen zwischen 18 und 65 Jahren bei Vorliegen einer ärztlich oder psychotherapeutisch aktuell oder im bisherigen Lebensverlauf diagnostizierten depressiven Erkrankung. Von der Teilnahme ausgeschlossen wurden Personen mit komorbiden organisch-symptomatisch psychischen Störungen, Personen mit Erkrankungen aus dem schizophrenen Formenkreis und Personen mit Störungen durch psychotrope Substanzen. Eine differenzierte Übersicht über die Ein- und Ausschlusskriterien der Studie (Phase 1b) findet sich in Tabelle 4.

Tabelle 4

Ein- und Ausschlusskriterien der Pilotstudie

\section{Einschlusskriterien}

Alter zwischen 18 und 55 Jahren*

Summenwert im Beck Depressions-Inventar $<20^{* *}$

Ärztlich oder psychotherapeutisch diagnostizierte depressive Erkrankung gemäß den Kriterien des ICD-10 jemals:
a) F31
bipolare affektive Störung
b) F32
depressive Episode
c) F33
rezidivierende depressive Störung
d) F34
anhaltend affektive Erkrankung (Zyklothymie, Dysthymie)
e) F38
andere affektive Störungen
f) F41.2 Angst und depressive Störung, gemischt
g) F43.2 Anpassungsstörungen

F43.20 kurze depressive Reaktion 
F43.21 längere depressive Reaktion

F43.22 Angst und depressive Reaktion gemischt

Schriftliches Einverständnis der Patienten nach ärztlicher Aufklärung.

Der Patient ist nach Einschätzung des Untersuchers in der Lage das Wesen und die Erfordernisse der Studie zu verstehen.

\section{Ausschlusskriterien}

Komorbiditäten, die nach ICD-10 als...

„F0: organische, symptomatische psychische Störungen“

„F1: Störungen durch psychotrope Substanzen“

„F2: Schizophrenie und wahnhafte Störungen“ klassifiziert werden, außer die F21-

Diagnose „schizotype Störung“.

Jemals schädlicher Substanzgebrauch und/oder -abhängigkeit gemäß ICD-Kriterien.

Anamnestisch bekanntes Schädel-Hirn-Trauma und/oder Bewusstlosigkeit > 5 Minuten in der Vorgeschichte.

Anamnestisch bekannte somatische/neurologische Erkrankung mit Auswirkungen auf das

Gehirn.

Drogengebrauch/-missbrauch innerhalb der letzten 4 Wochen.

Erhaltene Elektrokrampftherapie (EKT).

* im Rahmen der Pilotstudie wurde der Altersrange auf 18-65 Jahre ausgeweitet

** das Kriterium wurde im Rahmen der Pilotstudie uneingeschränkt erweitert

Insgesamt erklärten sich 85 Männer und Frauen bereit an der Studie teilzunehmen. Von den Analysen wurden 7 Personen aufgrund von schädlichem Substanzgebrauch oder neurologischen Erkrankungen mit Auswirkungen auf das Gehirn ausgeschlossen. Damit ergibt sich für die vorliegende Untersuchung eine Gesamtstichprobe von $N=78$ Teilnehmern. Für die Analysen werden die Studienteilnehmer ex post facto in Gruppen unterteilt. Von den 78 interviewten Teilnehmern sandten 58 (74.4\%) Personen die Fragebögen nach Bearbeitung ausgefüllt zurück. 


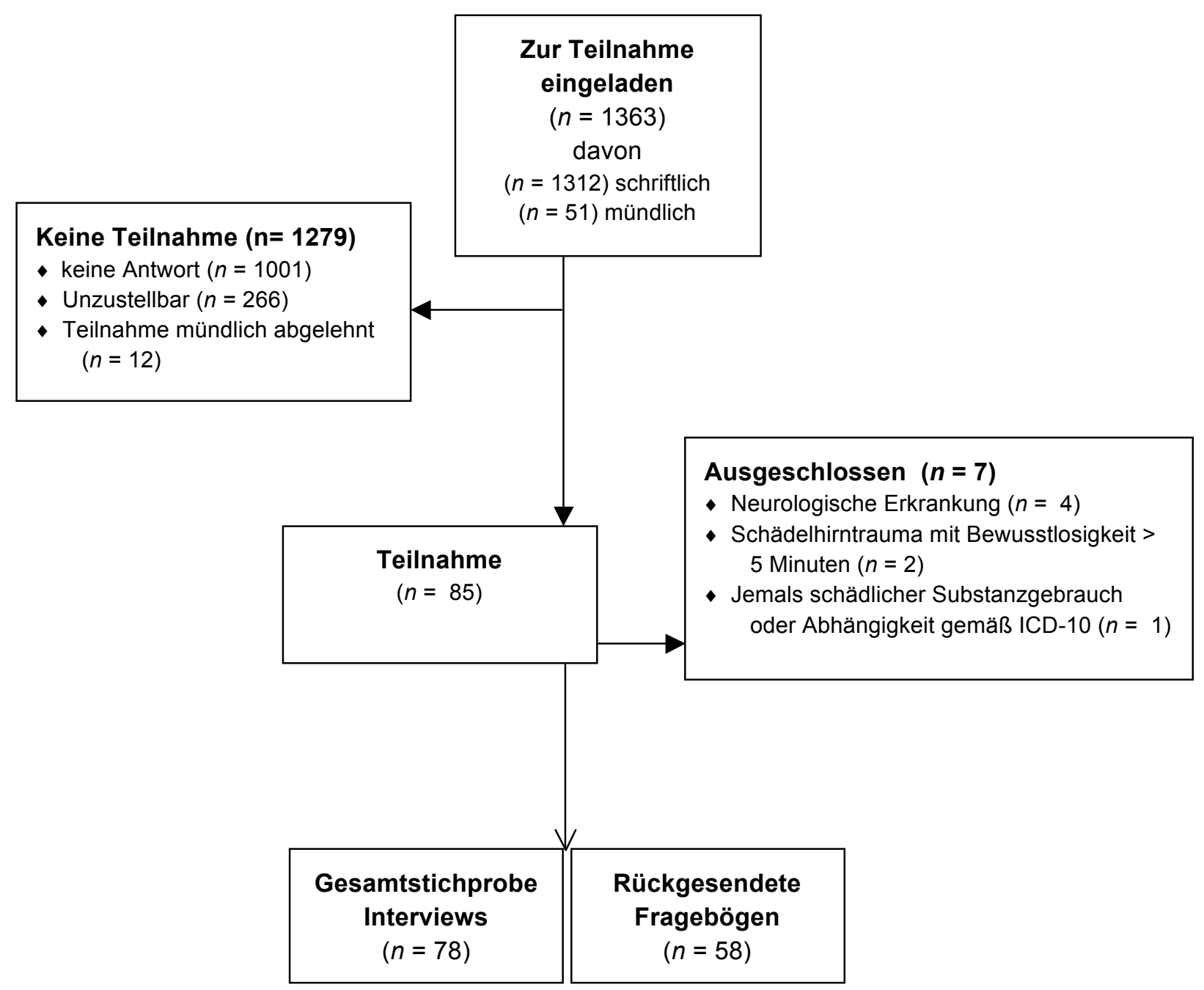

Abbildung 4. CONSORT Diagramm zur Stichprobengewinnung und entsprechende Fallzahlen im Rahmen der Pilotstudie. 


\subsection{Erhebungsinstrumente}

In der vorliegenden Studie wird ein klinisch-anamnestisches Interview zur Ätiopathogenese depressiver Erkrankungen entwickelt und zur Datengewinnung eingesetzt. Weiterhin werden vier Fragebögen zu Schutz- und Risikofaktoren sowie psychopathologischen Charakteristika der Prodromalphase konzipiert und zur Datenerhebung herangezogen. Daneben werden etablierte und standardisierte Fragebögen und Screeninginstrumente zur diagnostischen Einschätzung psychiatrischer Symptome verwendet.

Beck Depression Inventar II. Das Beck-Depressions Inventar II (BDI-II; Beck, Steer \& Brown, 1996) ist ein Selbstbeurteilungsverfahren zur Erfassung des Schweregrads depressiver Symptomatik bei psychiatrisch diagnostizierten Jugendlichen und Erwachsenen sowie ein Screeninginstrument für das Vorliegen depressiver Symptome in der Normalbevölkerung (Hautzinger, Keller \& Kühner, 2006). Im deutschen Sprachraum findet die Übersetzung nach Hautzinger, Bailer, Worall und Keller (1996) Anwendung. Die Symptome werden dabei für den Zeitraum der letzten zwei Wochen vor Befragung erhoben. Der Fragebogen umfasst insgesamt 21 Items, welche vierstufig skaliert sind (z.B. von $0=$ „ich bin nicht traurig bis $3=$,ich bin so traurig oder unglücklich, dass ich es nicht aushalte"). Höhere Werte stellen eine höhere Ausprägung bzw. einen höheren Schweregrad depressionsspezifischer Symptomatik dar. Die interne Konsistenz ist mit Werten über Cronbach $\alpha=.89$ als gut zu bewerten. Die Retestreliabilität des deutschsprachigen BDI-II wird mit $r=.78$ als sehr zufriedenstellend bewertet (Hautzinger et al., 2006). Die Validität des Verfahrens gilt als bestätigt (Hautzinger et al., 2006).

Depression Early Prediction - Interview. Das Depression Early Prediction Interview (DEEP-I) ist ein selbst generiertes (Stegmüller, Minkwitz, Ruhrmann, Schultze-Lutter, Schüle \& Meisenzahl, in Vorbereitung), halb-standardisiertes anamnestisches Interview, das sowohl soziodemographische Informationen als auch den Erkrankungsverlauf sowie die Einflussfaktoren der Entstehung depressiver Störungen erfasst. Die Durchführungsdauer des DEEP-I beläuft sich im Durchschnitt auf zwei Stunden. Das Interview umfasst die folgenden Sektionen:

Allgemeine soziodemographische Informationen. Erfasst werden Geschlecht, Alter, Gewicht, Größe, Raucherstatus jetzt und früher, Anzahl der Zigaretten pro Tag aktuell und 
früher, Händigkeit, Geburtsort, Nationalität, Muttersprache, Mehrsprachigkeit und Bildungsstand.

Familienstand und Wohnsituation. Erhoben werden der aktuelle Familienstand, die Anzahl der Kinder, das jeweilige Geburtsjahr der Kinder und die aktuelle Wohnsituation.

Ausbildung und Beruf. Erfasst werden Schuljahre insgesamt, höchster Schulabschluss, Berufstätigkeit aktuell, Tätigkeit/Studienfach aktuell, beruflicher Werdegang, Berentung/Pensionierung Ja/Nein und seit wann, Berufsgruppe aktuell, Nacht-/Schichtarbeit jemals/aktuell sowie monatliches Nettoeinkommen.

Somatische Beschwerden, Medikamenteneinnahme und Drogenkonsum. Die Beeinträchtigungen durch somatische Erkrankungen (Herzerkrankungen/ Herzkreislauferkrankungen, Bluthochdruck, Bluterkrankungen/Blutgefäßerkrankungen, Atemwegserkrankungen, Erkrankungen der Augen/Ohren/Nase/Kehlkopf, Erkrankungen des Magens/Zwölffingerdarms/Galle/Bauchspeicheldrüse, Darmerkrankungen, Lebererkrankungen, Nierenerkrankungen, Urologische/Gynäkologische Erkrankungen, Muskel- und Skelettsystem/Hauterkrankungen, Neurologische Erkrankungen, Endokrinologische/Stoffwechselerkrankungen) werden im DEEP-I auf einer fünfstufigen Likertskala (von 1 = "kein Problem" bis 5 = "lebensbedrohlich“) eingestuft. Weitere körperliche Erkrankungen/Beschwerden, chronische Erkrankungen und Arztbesuche sowie deren Häufigkeit in den letzten 12 Monaten werden im Ja/Nein Format erfragt. Die Sektion zur aktuellen Medikation sowie zur Einnahme von Psychopharmaka beinhaltet Fragen zu Wirkstoff, Dosierung, Einnahmeschemata und -zeitraum. Erfasst werden weiterhin aktueller Drogenkonsum (Substanz, Häufigkeit der Einnahme/Monat, Dosis, Einnahmezeitraum) und Drogen- bzw. Medikamenteneinnahme vor erstmaligem Auftreten einer depressiven Erkrankung (Substanz, Dosierung, Einnahmeschema, Einnahmezeitraum). Das Vorliegen eines Schädelhirntraumas mit Bewusstlosigkeit von einer Dauer über fünf Minuten in der Vergangenheit sowie einer neurologischen Erkrankung mit Auswirkung auf das Gehirn werden im Ja/Nein Format erfragt.

Allgemeine psychiatrische Anamnese. Erfasst werden hier die Inanspruchnahme medizinisch-psychiatrischer Versorgung jemals (stationär und ambulant, Zeitraum, Behandlungsgrund), medizinische Behandlungen aufgrund von Medikamenten-, Alkohol- 
oder Drogenproblemen, Selbstverletzung, Suizidversuche, psychische Erkrankungen in der Familie sowie Suizidversuche und Suizide bei Angehörigen.

Diagnose, Verlauf und Symptome der letzten Krankheitsphase und der letzten Prodromalphase. Erfasst werden körperliche und psychische Hauptsymptome der letzten, d.h. der zeitlich jüngsten Krankheitsphase und/oder Hauptsymptome der mnestisch repräsentativsten Erkrankungsphase, körperliche und psychische Frühsymptome, Beginn der Frühsymptome (Monat/Jahr), Zeitpunkt des Erstkontakts mit einer medizinisch/psychiatrischen Einrichtung (Monat/Jahr), die Art des Erstkontakts (Hausarzt, Psychiater, Neurologe, Psychologe, Psychotherapeut). Beginn und Dauer der Frühsymptome werden mit der Life-Chart Methode (Lyketsos et al., 1994) erhoben. Um Gedächtnisinhalte zu reaktivieren werden nach Lyketsos et al. (1994) Life Events vor Erkrankungsbeginn erfragt und im Anschluss die Frühsymptome sowie deren zeitlicher Verlauf bestimmt. Die Probanden werden zusätzlich gebeten ihren Krankheitsverlauf in einem Zeitstrahl auf einem vorgelegtem DinA4 Format graphisch darzustellen. Die Achsen Zeit und Symptomausprägung (von -7 „gedrückte Stimmung, verminderter Antrieb, Freund- und Interessenlosigkeit“; bis +7 „übermäßig gehobene Stimmung“, „vermehrter Antrieb“) sind dabei im Interviewbogen vorgegeben, die Einteilung und Verankerung auf der Zeitachse erfolgt individuell durch die Probanden. Beginn und Ende von Krankheitsphase und Prodromalphase werden mit den Fragen „Wann begann Ihre letzte Krankheitsphase?“, „welche psychischen Frühsymptome bemerkten Sie?“, „welche körperlichen Frühsymptome bemerkten Sie?“, „zu welchem Zeitpunkt bemerkten Sie früheste Anzeichen oder Veränderungen vor der letzten Krankheitsphase?“, „wann nahmen Sie erstmalig Kontakt zu einer medizinischpsychiatrischen Einrichtung auf?" erfasst.

Spezifische psychiatrische Anamnese zur Depression. Erfragt werden zusätzlich die Anzahl diagnostizierter und nicht diagnostizierter Erkrankungsphasen der Depression im Lebensverlauf und der jeweilige Erkrankungszeitraum (Beginn, Monat/Jahr; Ende, Monat/Jahr).

Depression Early Prediction - Fragebogen. Der Depression Early Prediction Fragebogen (DEEP) ist ein selbst generierter (Stegmüller, Minkwitz, Ruhrmann, Schultze-Lutter, Schüle \& Meisenzahl, in Vorbereitung) Fragebogen zur Selbstauskunft, der auf Grundlage der dargestellten ätiopathogenetischen Modelle depressiver Erkrankungen und des abgeleiteten 
vierfaktoriellen Entstehungsmodells (siehe Abbildung 2) entwickelt wurde. Der DEEPFragebogen setzt sich aus den vier einzelnen Fragebögen DEEP-Prodromalsymptome (DEEPA), DEEP-Stressoren (DEEP-B), DEEP-Ressourcen (DEEP-C) und DEEP-Vulnerabilität (DEEP-D) zusammen. Nachfolgend findet sich eine Aufstellung und detaillierte Beschreibung der einzelnen Inhalte. Die Quellenangaben finden sich in einem separaten Literaturverzeichnis am Ende der Arbeit.

DEEP - Prodromalsymptome (DEEP-A). Der DEEP-A wurde als Fragebogen zur Selbstauskunft konzipiert und erfasst retrospektiv erste Anzeichen einer depressiven Erkrankung auf Symptomebene im Rahmen der Prodromalphase. Der Fragebogen enthält 91 Items, welche die Konstrukte Veränderung in Antrieb, Belastbarkeit und Stimmung, depressive und manische Frühsymptome, qualitativ berichtete subjektive Frühsymptome, psychosoziale Belastung, somatische Symptome sowie Appetit und Essverhalten abbilden. Die Fragen wurden in Anlehnung an die in Tabelle 5 aufgeführten psychometrischen Instrumente generiert und formuliert. Die Symptome werden dabei für den Zeitraum vor Erkrankungsbeginn erfasst. Der Prodromalzeitraum bestimmt sich interindividuell aus dem DEEP-Interview und wird vor Fragbogenbearbeitung mittels Life-Chart Methode verankert. Die Items sind siebenstufig (von $0=$ „überhaupt nicht“ bis $6=$ „sehr stark“) oder dreistufig (von $0=$ „nicht beeinträchtigt“ bis 2 = „stark beeinträchtigt“) skaliert. Höhere Werte stellen dabei eine höhere Ausprägung der depressionsspezifischen Frühsymptomatik dar. Einzelne Items erfordern Ja/Nein Antworten, wie zum Beispiel die Veränderung der Stimmung bedingt durch den weiblichen Monatszyklus. Die Fragen zu Appetit und Essverhalten wurden gesondert fünf- bzw. dreistufig nach Vorlage des Simplified Nutritional Appetite Questionnaire (SNAQ; Wilson et al., 2005) skaliert. 
Tabelle 5

DEEP-A: abgebildete Konstrukte, Itemanzahl und Quellen der Itemgenerierung

\begin{tabular}{|c|c|c|c|}
\hline Konstrukt & Itemanzahl & $\begin{array}{l}\text { Formuliert in Anlehnung } \\
\text { an... }\end{array}$ & Kurzbezeichnung \\
\hline $\begin{array}{l}\text { Veränderung in Antrieb, } \\
\text { Belastbarkeit und } \\
\text { Stimmung }\end{array}$ & 23 & $\begin{array}{l}\text { Schizophrenia Proneness } \\
\text { Instrument }^{1}\end{array}$ & $\mathrm{SPI}-\mathrm{CY}$ \\
\hline $\begin{array}{l}\text { Depressive } \\
\text { manische } \\
\text { Frühsymptome }\end{array}$ & 35 & $\begin{array}{l}\text { Bipolar Prodrome } \\
\text { Symptom Interview }\end{array}$ & BPSS-P \\
\hline $\begin{array}{l}\text { Qualitative } \\
\text { Frühsymptome }\end{array}$ & 3 & $\begin{array}{l}\text { Depression Early } \\
\text { Prediction - Prodrome } \\
\text { Questionnaire }^{3}\end{array}$ & DEEP \\
\hline Psychosoziale Belastung & 10 & $\begin{array}{ll}\text { Patient } & \text { Health } \\
\text { Questionnaire }^{4} & \end{array}$ & PHQ-D \\
\hline Somatische Symptome & 13 & $\begin{array}{ll}\text { Patient } & \text { Health } \\
\text { Questionnaire } & \\
\end{array}$ & PHQ-D \\
\hline $\begin{array}{l}\text { Veränderung der } \\
\text { Stimmung zyklusbedingt }\end{array}$ & 2 & $\begin{array}{ll}\text { Patient } & \text { Health } \\
\text { Questionnaire } & \\
\end{array}$ & PHQ-D \\
\hline $\begin{array}{ll}\text { Appetit } & \text { und } \\
\text { Essverhalten } & \end{array}$ & 5 & $\begin{array}{l}\text { Simplified Nutritional } \\
\text { Appetite Questionnaire }^{5}\end{array}$ & SNAQ \\
\hline Items gesamt & 91 & DEEP-Prodrome & DEEP-A \\
\hline
\end{tabular}

DEEP - Stressoren (DEEP-B). Der DEEP-B wurde als Fragebogen zur Selbstauskunft konzipiert und erfasst retrospektiv Stressoren und Belastungsfaktoren in der Ätiopathogenese depressiver Erkrankungen. Der Fragebogen enthält 285 Items, welche die Konstrukte Burn-Out, chronischer Stress, Alltagsbelastungen, Lebensereignisse, wahrgenommene Selbstbestimmung, Mobbingerfahrung, wahrgenommene Diskriminierung und psychosoziale Beeinträchtigung abbilden. Die Fragen wurden in Anlehnung an die in Tabelle 6 aufgeführten psychometrischen Instrumente formuliert.

\footnotetext{
${ }^{1}$ Schultze-Lutter \& Koch (2010)

${ }^{2}$ Correll et al. (2014)

${ }^{3}$ Stegmüller et al. (in Vorbereitung)

${ }^{4}$ Löwe (2001)

${ }^{5}$ Wilson et al. (2005)
} 
Tabelle 6

DEEP-B: abgebildete Konstrukte, Itemanzahl und Quellen der Itemgenerierung

\begin{tabular}{|l|l|l|l|}
\hline Konstrukt & Itemanzahl & $\begin{array}{l}\text { Formuliert in Anlehnung } \\
\text { an... }\end{array}$ & Kurzbezeichnung \\
\hline Burn-Out & 25 & Maslach Burnout Interview & MBI \\
\hline Chronischer Stress & 57 & $\begin{array}{l}\text { Trier Inventar zum } \\
\text { Chronischen Stress }\end{array}$ & TICS \\
\hline Alltagsbelastungen & 61 & $\begin{array}{l}\text { Alltags-Belastungs- } \\
\text { Fragebogen }^{8}\end{array}$ & ABF \\
\hline Lebensereignisse & 83 & Münchner Ereignisliste $^{9}$ & MEL \\
\hline $\begin{array}{l}\text { Wahrgenommene } \\
\text { Selbstbestimmung }\end{array}$ & 10 & $\begin{array}{l}\text { Self-Determination Scale } \\
\text { (10 }\end{array}$ & SDS \\
\hline Mobbingerfahrung & 12 & Trier Mobbing Kurzskala & TMKS \\
\hline $\begin{array}{l}\text { Wahrgenommene } \\
\text { Diskriminierung }\end{array}$ & 9 & $\begin{array}{l}\text { Everyday Discrimination } \\
\text { Scale }\end{array}$ & EDS \\
\hline $\begin{array}{l}\text { Psychosoziale } \\
\text { Beeinträchtigung }\end{array}$ & 28 & Predict D ${ }^{13}$ & Predict D \\
\hline Items gesamt & $\mathbf{2 8 5}$ & & DEEP-B \\
\hline
\end{tabular}

DEEP - Ressourcen (DEEP-C). Der DEEP-C wurde als Fragebogen zur Selbstauskunft konzipiert und erfasst retrospektiv vorhandene Schutzfaktoren im Sinne von Resilienz, Ressourcen und Copingstrategien in der Ätiopathogenese depressiver Erkrankungen. Der Fragebogen enthält 283 Items, welche die Konstrukte subjektive Lebensqualität, soziale Unterstützung, Lebenszufriedenheit, Bewältigungs- und Verarbeitungsmaßnahmen in belastenden Situationen, Kontrollüberzeugungen, generalisierten Optimismus/Pessimismus, gesunde Ernährung, Sport und Freizeitaktivitäten, Spiritualität, Religiosität und Resilienz abbilden sollen. Die Fragen wurden in Anlehnung an die in Tabelle 7 aufgeführten psychometrischen Instrumente formuliert.

\footnotetext{
${ }^{6}$ Büssing \& Perrar (1992)

${ }^{7}$ Schulz, Becker \& Schlotz (2004)

${ }^{8}$ Traue, Hrabal \& Kosarz (2000)

${ }^{9}$ Maier-Diewald (1983)

${ }^{10}$ Sheldon (1995)

${ }^{11}$ Klusemann, Nikolaides, Schwickerath, Brunn \& Kneip (2008)

${ }^{12}$ Forman, Williams \& Jackson (1997)

${ }^{13}$ King, Walker, Levy, Bottomley et al. (2008)
} 
Tabelle 7

DEEP-C: abgebildete Konstrukte, Itemanzahl und Quellen der Itemgenerierung

\begin{tabular}{|c|c|c|c|}
\hline Konstrukt & Itemanzahl & Formuliert in Anlehnung an... & Kurzbezeichnung \\
\hline $\begin{array}{l}\text { Subjektive } \\
\text { Lebensqualität }\end{array}$ & 26 & $\begin{array}{l}\text { World Health Organisation } \\
\text { Quality of Life }{ }^{14}\end{array}$ & WHOQOL-BREF \\
\hline Soziale Unterstützung & 12 & $\begin{array}{l}\text { Mulitdimensional Scale } \\
\text { Perceived Social Support }^{15}\end{array}$ & MSPSS \\
\hline Lebenszufriedenheit & 10 & $\begin{array}{l}\text { Brief Multidimensional Life } \\
\text { Satisfaction Scale }{ }^{16}\end{array}$ & BMLSS \\
\hline $\begin{array}{l}\text { Bewältigungs- und } \\
\text { Verarbeitungsmaß- } \\
\text { nahmen in belastenden } \\
\text { Situationen }\end{array}$ & 120 & Stressverarbeitungsfragebogen ${ }^{17}$ & SVF-120 \\
\hline Kontrollüberzeugungen & 32 & $\begin{array}{l}\text { Fragebogen zu Kompetenz- und } \\
\text { Kontrollüberzeugung }{ }^{18}\end{array}$ & FKK \\
\hline $\begin{array}{l}\text { Generalisierter } \\
\text { Optimismus/ } \\
\text { Pessimismus }\end{array}$ & 10 & Life-Orientation-Test $^{19}$ & LOT-R \\
\hline Gesunde Ernährung & 8 & Starting The Conversation ${ }^{20}$ & STC-Diet \\
\hline $\begin{array}{ll}\text { Sport } & \text { und } \\
\text { Freizeitaktiviäten } & \end{array}$ & 15 & Sport und Freizeitaktivitäten & DEEP-C \\
\hline Spiritualität & 12 & $\begin{array}{l}\text { Functional Assessment of } \\
\text { Chronic Illness Therapie - } \\
\text { Spiritual Wellbeing }\end{array}$ & FACIT-Sp-12 \\
\hline Religiosität & 5 & Idler Index of Religiosity ${ }^{22}$ & IIR \\
\hline Resilienz & 33 & Resilience Scale for Adults ${ }^{23}$ & RSA \\
\hline Items gesamt & 283 & & DEEP-C \\
\hline
\end{tabular}

DEEP - Vulnerabilität (DEEP-D). Der DEEP-D wurde als Fragebogen zur Selbstauskunft konzipiert und erfasst retrospektiv Vulnerabilitätsfaktoren in der Ätiopathogenese depressiver Erkrankungen. Der Fragebogen enthält 267 Items, welche die Konstrukte Temperament, traumatische Lebensereignisse, Perfektionismus, Attributionsstil, Alkoholkonsum, Drogenkonsum, Nikotinkonsum, Ruminationsneigung, Selbstwertgefühl,

\footnotetext{
${ }^{14}$ WHOQOL Group. (1998)

${ }^{15}$ Zimet, Dahlem, Zimet \& Farley (1988)

${ }^{16}$ Huebner, Suldo, Valois, Drane \& Zullig (2004)

17 Janke, Erdmann \& Boucsein (1985)

${ }^{18}$ Krampen (1991)

${ }^{19}$ Glaesmer, Hoyer, Klotsche \& Herzberg (2008)

${ }^{20}$ Paxton, Strycker, Toobert, Ammerman \& Glasgow (2011)

${ }^{21}$ Bredle, Salsman, Debb, Arnold \& Cella (2011)

${ }^{22}$ Idler (1987)

${ }^{23}$ Hjemdal et al. (2011)
} 
essensbezogene Verhaltensweisen und Kognitionen sowie Persönlichkeitsausprägungen abbilden. Die Fragen wurden in Anlehnung an die in Tabelle 8 aufgeführten psychometrischen Instrumente formuliert. Die Fragebögen zu den Konstrukten Temperament, traumatische Erfahrungen in der Kindheit, Perfektionismus und Persönlichkeitsausprägungen wurden nicht retrospektiv umformuliert und die Probanden nicht zeitlich vor den Beginn der depressiven Erkrankung verankert. Die Instruktion enthielt eine Bezugnahme auf den gesamten Lebensverlauf und die Person im Allgemeinen. Für die vorliegenden Analysen wurden insbesondere die Skalenwerte Neurotizismus und Extraversion des Neo-Fünf-Faktoren-Inventars nach Costa und McCrae herangezogen (NEOFFI, Borkenau \& Ostendorf, 2008). Die Skala Neurotizismus des NEO-FFI enthält insgesamt 12 Items welche interindividuelle Unterschiede in der emotionalen Stabilität und der emotionalen Labilität von Personen erfassen. Die Skala Extraversion enthält 12 Items, welche die Dimensionen Geselligkeit, Selbstsicherheit, Aktivität, Gesprächigkeit, Energie, Heiterkeit und Optimismus abdecken. Die Items des NEO-FFI sind jeweils fünfstufig skaliert (von „starke Ablehnung“ bis „starke Zustimmung“). Die Skalenwerte werden als gewichtete Mittelwerte der jeweils zugehörigen Items berechnet und weisen einen Wertebereich zwischen null und 48 auf. Eine Beschreibung der psychometrischen Daten des NEO-FFI findet sich im Manual zum Fragebogen von Borkenau und Ostendorf (2008). Von weiterem Interesse waren die Skalenwerte des Childhood Trauma Questionnairs (CTQ). Der Fragebogen enthält insgesamt 25 Items, welche retrospektiv emotionalen, körperlichen und sexuellen Missbrauch sowie emotionale und körperliche Vernächlässigung im Kindes- und Jugendalter erfassen (Gast, Rodewald, Benecke \& Driessen, 2001). Die Items sind jeweils fünfstufig skaliert (von „überhaupt nicht“ bis „sehr häufig“). Höhere Werte bilden ein größeres Ausmaß an Misshandlung oder Vernachlässigung ab. Die Auswertung erfolgt über die Berechnung der Mittelwerte über die einzelen Skalen oder der Bildung eines Gesamtmittelwertes. Die teststatistischen Kennwerte der deutschen Version gelten als vergleichbar gut wie das amerikanische Original (Wingenfeld et al., 2010). Eine Übersicht zu den psychometrischen Kennwerten des Instruments findet sich bei Wingenfeld et al. (2010). 
Tabelle 8

DEEP-D: abgebildete Konstrukte, Itemanzahl und Quellen der Itemgenerierung

\begin{tabular}{|c|c|c|c|}
\hline Konstrukt & Itemanzahl & $\begin{array}{l}\text { Formuliert in Anlehnung } \\
\text { an... }\end{array}$ & Kurzbezeichnung \\
\hline Alkoholkonsum & 11 & $\begin{array}{l}\text { Alcohol Use Identification } \\
\text { Test }^{24}\end{array}$ & AUDIT \\
\hline Drogenkonsum & 13 & $\begin{array}{l}\text { Drug Use Disorder } \\
\text { Identification Test }^{25}\end{array}$ & DUDIT \\
\hline Nikotinkonsum & 6 & Fagerström Test $^{26}$ & Fagerström \\
\hline Rumination & 32 & $\begin{array}{ll}\text { Response } & \text { Style } \\
\text { Questionnaire }^{27} & \end{array}$ & RSQ-D \\
\hline Selbstwertgefühl & 10 & Self-Esteem Scale ${ }^{28}$ & SES \\
\hline Essstörung Screening & 26 & Eating Attitudes Test $26^{29}$ & EAT-26 \\
\hline Attributionsstil & 8 & $\begin{array}{l}\text { Attributionsstilfragebogen } \\
\text { für Erwachsene } \\
\text { fo }\end{array}$ & ASF-E \\
\hline Persönlichkeitsausprägungen* & 60 & $\begin{array}{l}\text { NEO-Fünf-Faktoren- } \\
\text { Inventar }^{31}\end{array}$ & NEO-FFI \\
\hline Temperament* & 35 & $\begin{array}{ll}\text { Temperament } & \text { Auto- } \\
\text { Questionnaire }^{32} & \end{array}$ & briefTEMPS-M \\
\hline $\begin{array}{l}\text { Traumatische } \\
\text { Lebensereignisse* }\end{array}$ & 31 & $\begin{array}{ll}\text { Childhood } & \text { Trauma } \\
\text { Questionnaire }^{33} & \end{array}$ & CTQ \\
\hline Perfektionismus* & 35 & $\begin{array}{l}\text { Frost Multidimensional } \\
\text { Perfectionism Scale }\end{array}$ & FMPS \\
\hline Items gesamt & 267 & & DEEP-D \\
\hline
\end{tabular}

* keine zeitliche Verankerung vor den Beginn der depressiven Erkrankung.

Die Skalenbildung der DEEP-Fragebögen sowie die Berechnung der psychometrischen Kennwerte sind in einer Folgestudie zur Fragebogenevaluation angestrebt. Eine Überprüfung der dargestellten Fragestellungen, welche sich aus den DEEP-Fragebögen herleiten, erfolgt in der vorliegenden Studie auf Grundlage der Auswertung von Einzelitems und der validierten Form der dargestellten Fragebögen. Die nachfolgenden Analysen beziehen sich

\footnotetext{
${ }^{24}$ Saunders et al. (2005)

${ }^{25}$ Berman, Bergmann, Palmsierna \& Schlyter (2005)

${ }^{26}$ Heatherton, Kozlowski, Frecker \& Fagerstrom (1991)

${ }^{27}$ Kühner, Huffziger \& Nolen-Hoeksema (2007)

${ }^{28}$ Pullman \& Allik (2000)

${ }^{29}$ Garner \& Garfinkel (1979)

${ }^{30}$ Poppe, Pelster \& Stiensmeier-Pelster (2005)

${ }^{31}$ Borkenau \& Ostendorf (2008)

${ }^{32}$ Erfurt, Gerlach, Hellweg et al. (2005)

${ }^{33}$ Gast, Rodewald, Benecke \& Driessen (2001)

${ }^{34}$ Stöber (2003)
} 
hauptsächlich auf das DEEP-Interview, den DEEP-A und den DEEP-D Fragebogen. Die Ergebnisse weiterer Fragestellungen, die sich auf die Fragebögen DEEP-B und DEEP-C beziehen, werden im Rahmen weiterer Arbeiten berichtet.

\subsection{Operationalisierung}

Altersgruppe. Anhand des Alters der Probanden zum Zeitpunkt der Datenerhebung wurde eine Einteilung in Altersgruppen vorgenommen. Die Zuteilung von Altersgruppen zu Geburtskohorten erfolgte in Anlehnung an die Geburtskohorteneinteilung nach Kessler et al. (2010). Damit ergaben sich folgende Kategorisierungen: Die jüngste Altersgruppe mit 18-34Jährigen, die mittlere Gruppe mit 35-49-Jährigen und die älteste Gruppe mit 50-64-Jährigen.

Verlauf der Erkrankung. Der Verlauf der Erkrankung wurde durch ein Item im DEEPInterview kodiert und in Erstmanifestation und rezidivierender Verlauf differenziert. Bei Vorliegen von mindestens zwei unabhängigen depressiven Episoden mit Vollremission im Intervall wurden Probanden der Gruppe rezidivierender Verlauf zugeordnet.

Anzahl der bisherigen Episoden. Die Anzahl der bisherigen depressiven Episoden im Lebensverlauf wurde aus der Addition der Angabe Anzahl frühere Episoden und der berichteten aktuellen oder letzten Episode erstellt.

Ersterkrankungsalter. Die Variable wurde für die vorliegenden statistischen Analysen als kontinuierliche Variable operationalisiert. Das Ersterkrankungsalter wurde im Rahmen des DEEP-Interviews aus dem Geburtsdatum und den Angaben aus dem Item Beginn der ersten Krankheitsphase, Monat/Jahr errechnet. Für weiterführende Analysen wurde das Ersterkrankungsalter getrennt für die ex post facto gebildeten Gruppen diagnostizierte Erstepisode und nicht diagnostiziert Erstepisode berechnet. Das im DEEP-Interview enthaltene Item Beginn der ersten Krankheitsphase beinhaltet den Zusatz diagnositziert Ja/Nein.

Dauer der letzten Prodromalphase. Die Dauer der letzten Prodromalphase wurde über die zeitliche Differenz zwischen dem Auftreten des ersten Frühsymptoms der depressiven Erkrankung und dem subjektiven Beginn der Erkrankung mit Vollausprägung der Symptome 
sowie einer Beeinträchtigung im Funktionsniveau definiert und errechnet. Die Dauer der Prodromalphase wurde jeweils in Monaten berechnet.

Symptome der Prodromalphase. Die im freien Abruf berichteten psychischen und körperlichen Frühsymptome wurden ex post facto in Kategorien unterteilt. Die Kategorienbildung erfolgte theoriegeleitet und anhand der Häufung inhaltlich übereinstimmend berichteter Symptome.

Dauer der unbehandelten Erkrankung (DUI), Dauer der letzten Episode (DUE). Die Dauer der unbehandelten Erkrankung und die Dauer der letzten Episode wurden definiert als die zeitliche Differenz zwischen dem Beginn der letzten Erkrankungsphase mit voll ausgeprägter Symptomatik und dem Erstkontakt mit einer medizinisch-psychiatrischen Einrichtung während der letzten Krankheitsphase. Die Dauer wurde jeweils in Monaten berechnet.

\subsection{Design}

Bei vorliegender Studie handelt es sich um eine Fragebogenentwicklung und Machbarkeitsstudie bei retrospektiver Querschnittuntersuchung mit einmaliger Befragung der Studienteilnehmer. Für sekundäre Analysen wurden die Studienteilnehmer ex post facto in Gruppen unterteilt. Eine Unterteilung erfolgte nach Geschlecht, Altersgruppen, Krankheitsverlauf und aktueller depressiver Symptomatik. Es handelt sich um eine Pilotstudie im Rahmen eines mehrstufig konsekutiven Projekts (siehe Abbildung 3).

\subsection{Durchführung}

Die Datenerhebung erfolgte am Interdisziplinären Centrum für Prävention Psychischer Erkrankungen (CEP ${ }^{\mathrm{LMU}}$ ) der Ludwig-Maximilians-Universität in München, welches an die Psychiatrische Klinik der Universität in München angegliedert ist. Das Erhebungsvorhaben wurde von der Ethikkommission der Medizinischen Fakultät der Ludwig-MaximiliansUniversität begutachtet und gemäß der Helsinki Deklaration für unbedenklich befunden (EK 542-15). Die Rekrutierung der Probanden erfolgte durch schriftliche oder mündliche Einladung der Patienten im stationären und ambulanten Versorgungsbereich des LMU 
Klinikums für Psychiatrie und Psychotherapie in München sowie durch Aushänge zur Studie an der CIP-Tagesklinik in München. Eine Übersicht über das Erhebungsverfahren findet sich in Abbildung 5. Schriftlich zur Teilnahme eingeladen wurden Patienten des Klinikums für Psychiatrie und Psychotherapie in München, die im Zeitraum von Januar 2012 bis Dezember 2015 aufgrund einer depressiven Erkrankung stationär oder ambulant behandelt worden waren. Mündlich angesprochen wurden Patienten, welche sich im Erhebungszeitraum von Oktober 2015 bis August 2016 aufgrund einer depressiven Erkrankung in ambulanter, stationärer oder teilstationärer Behandlung am LMU Klinikum für Psychiatrie und Psychotherapie befanden. In der CIP Tagesklinik in München wurden Informationsblätter zur Studie und eine Einladung zur Teilnahme ausgelegt. Sowohl die mündliche Kontaktaufnahme als auch das Anschreiben beinhalteten eine Einladung zur Teilnahme an der Studie und eine Aufklärung über den Zweck der Untersuchung. Enthalten waren ebenso Informationen zur Gewährleistung von Anonymität und Datenschutz sowie die entsprechenden Kontaktdaten für Rückfragen und Anmeldung zur Studie. An der Teilnahme Interessierte wurden zum Interview eingeladen und gebeten einen Arztbrief mitzubringen, aus welchem die Diagnose einer depressiven Störung hervorgehe. Die Studienteilnahme erfolgte nach umfassender Aufklärung und schriftlicher Einverständniserklärung der Patienten. Die Teilnahme beinhaltete ein etwa zweistündiges anamnestisches Interview zu Entstehung und Verlauf der depressiven Erkrankung sowie das selbständige Ausfüllen von insgesamt fünf Fragebögen. Während des Interviews wurde die letzte bzw. mnestisch repräsentativste depressive Episode mittels Life-Chart-Methode verankert und die Studienteilnehmer instruiert die nachfolgenden Fragebögen aus der Perspektive vor Beginn dieser Erkrankungsphase zu beantworten. Die Fragebögen wurden den Studienteilnehmern in vorfrankierten Umschlägen mitgegeben, mit dem Hinweis die Rücksendung ohne Absenderadresse vorzunehmen, um den Datenschutz und die Anonymität zu gewährleisten. Die Probanden erhielten nach Teilnahme am Interview und bei Rücksendung der ausgefüllten Fragebögen eine von der Ethikkommission der Medizinischen Fakultät in München genehmigte Aufwandsentschädigung in Höhe von 40,- Euro. Alle interessierten Patienten, die sich zur Teilnahme bereit erklärten wurden im Zeitraum von Oktober 2015 bis August 2016 befragt. 


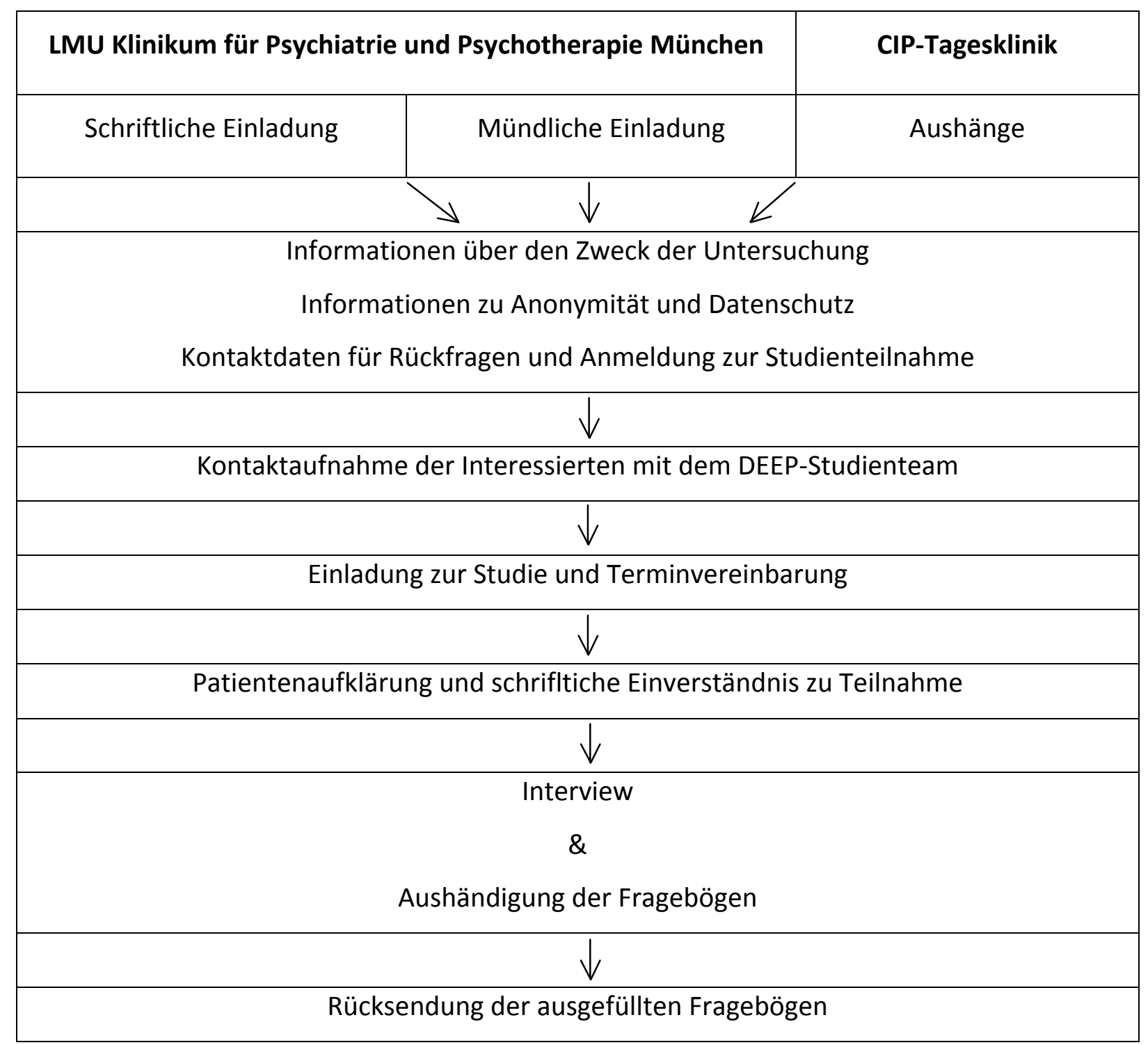

Abbildung 5. Ablauf des Datenerhebungsverfahrens in der Pilotstudie (Phase 1b). 


\subsection{Statistische Analysen}

Die statistischen Analysen wurden mit dem Statistikprogramm IBM SPSS 22 durchgeführt. Zur Stichprobenbeschreibung wurden deskriptive Analysen herangezogen. Häufigkeitsunterschiede wurden mittels Chi-Quadrat Tests auf statistische Signifikanz überprüft. Im Falle geringer Zellbesetzung wurden die Signifikanzwerte des Fisher-Exakt Tests angegeben. Häufigkeitsverteilungen mehrfach gestufter kategorialer Variablen wurden anhand loglinearer Modelle auf Haupt- und Interaktionseffekte überprüft. Interaktionseffekte wurden mittels Chi-Quadrat Tests in Einzelvergleiche aufgebrochen und analysiert. Bei geringer Zellbesetzung wurden die Signifikanzwerte des Fisher-Exakt Tests angegeben. Quotenverhältnisse wurden anhand von Odds Ratios berechnet, welche die Chancenverhältnisse eines Merkmals in zwei Gruppen vergleichen. Je höher der Odds Ratio ist, desto höher ist die Chance des Auftretens eines Merkmals in einer Gruppe im Vergleich zu einer anderen. Die Überprüfung von Zusammenhangshypothesen erfolgte mittels bivariaten Pearson-Korrelationskoeffizienten und linearen Regressionsanalysen. Die standardisierten Regressionskoeffizienten repräsentieren jeweils das Ausmaß der Veränderung in der abhängigen Variablen, das mit einer Veränderung in der unabhängigen Variablen um eine Standardabweichung einhergeht. Gruppenunterschiede wurden mittels tTests für unabhängige Stichproben und univariaten sowie multivariaten Varianzanalysen geprüft. Für alle univariaten und multivariaten Analysen wurde der approximierte F-Wert als Prüfgröße angegeben. Interaktionseffekte wurden anhand von Simple Effects Analysen untersucht. Post-hoc Analysen wurden mittels Bonferroni-Tests ( $p$-level .05) durchgeführt.

Die Berechnung der Effektstärken erfolgte im Programm G*Power (Faul, Erdfelder, Buchner, \& Lang, 2009). Der Wert des Effektstärkemaßes Cohen's $d$ ist ab einem Wert von .20 als kleiner Effekt zu beurteilen. Ab einem Wert von .50 ist von einem mittleren und ab einem Wert von .80 von einem großen Effekt auszugehen. Im Effektstärkemaß Cohen's $f$ beschreiben Werte $a b .10$ einen kleinen Effekt, Werte $a b .25$ einen mittleren und Werte $a b$ .40 einen großen Effekt. 


\section{Ergebnisse}

\subsection{Stichprobenbeschreibung}

Diagnosen. Von den insgesamt 78 Studienteilnehmern befanden sich 63 (80.8\%) Personen im Zeitraum zwischen Januar 2012 und August 2016 aufgrund einer depressiven Erkrankung am LMU Klinikum in Behandlung, davon 33 (42.3\%) Probanden im stationären und 30 (38.5\%) im ambulanten Setting. Durch externe Aushänge wurden 15 (19.2\%) Personen zur Studienteilnahme gewonnen. Bei allen 78 Probanden war eine ärztlich diagnostizierte depressive Erkrankung, eine Anpassungsstörung oder eine Angst und depressive Störung gemischt im Lebensverlauf nachweisbar. Für alle Probanden lag ein Arztbrief vor, aus dem die Diagnose einer depressiven Erkrankung entsprechend der dargestellten Einschlusskriterien nachvollzogen werden konnte. Eine Übersicht über die Verteilung der Hauptdiagnosen nach ICD-10 findet sich in Tabelle 9. Am häufigsten vertreten waren die Diagnosen der schweren rezidivierenden depressiven Störung (42.2\%) und der schweren depressiven Episode (17.6\%).

Tabelle 9

Verteilung der Hauptdiagnosen in der Gesamtstichprobe $(N=78)$

\begin{tabular}{lllc}
\hline Hauptdiagnosen nach ICD-10 & $N$ & $\%$ \\
\hline F31.4 & Bipolar affektive Störung, gegenwärtig schwere depressive Episode ohne & 1 & 1.2 \\
& psychotische Symptome & & \\
F31.5 & Bipolare affektive Psychose, gegenwärtig schwere depressive Episode mit & 1 & 1.2 \\
& psychotischen Symptomen & & \\
F32.0 & Leichte depressive Episode & 1 & 1.2 \\
F32.1 & Mittelgradige depressive Episode & 9 & 10.6 \\
F32.2 & Schwere depressive Episode ohne psychotische Symptome & 15 & 17.6 \\
F33.0 & Rezidivierende depressive Störung, gegenwärtig leichte Episode & 4 & 4.7 \\
F33.1 & Rezidivierende depressive Störung, gegenwärtig mittelgradige Episode & 10 & 11.8 \\
F33.2 & Rezidivierende depressive Störung, gegenwärtig schwere Episode ohne & 36 & 42.2 \\
& psychotische Symptome & &
\end{tabular}

F33.3 Rezidivierende depressive Störung, gegenwärtig schwere Episode mit $2 \quad 2.4$ 
psychotischen Symptomen

F33.4 Rezidivierende depressive Störung, gegenwärtig remittiert $\quad 1 \quad 1.2$

F41.2 Angst und depressive Störung, gemischt $\quad 2 \quad 2.4$

F43.2 Anpassungsstörung 112

Z73 Probleme mit Bezug auf Schwierigkeiten bei der Lebensbewältigung $\quad \begin{array}{lll}1 & 1.2\end{array}$

Alter und Geschlecht. Das durchschnittliche Alter der Studienteilnehmer in der Gesamtstichprobe $(N=78)$ betrug 39.4 Jahre $(S D=10.32)$. Insgesamt 27 (34.6\%) Probanden fielen in die Altersgruppe der 18-34-Jährigen, 36 (46.2\%) Probanden in die Altersgruppe der 35-49-Jährigen und 15 (19.2\%) Probanden in die Altersgruppe der 50-64-Jährigen. Der Wertebereich des Alters lag in der Gesamtstichprobe zwischen 20 und 63 Jahren. Die Geschlechterverteilung belief sich auf 49 (62.8\%) weibliche und 29 (37.2\%) männliche Studienteilnehmer. In Abbildung 6 und Abbildung 7 sind die Häufigkeitsverteilungen der Studienteilnehmer nach Altersgruppen und Geschlecht dargestellt.

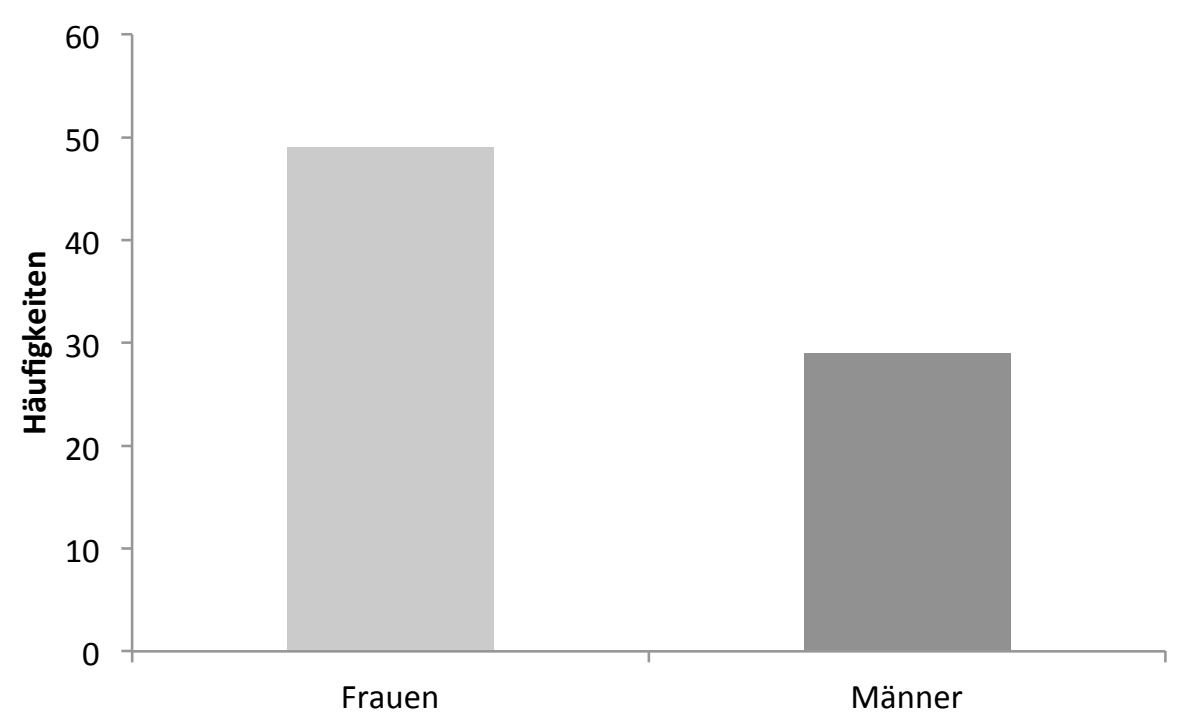

Abbildung 6. Häufigkeitsverteilung der Studienteilnehmer getrennt für Männer und Frauen in der Gesamtstichprobe $(N=78)$. 


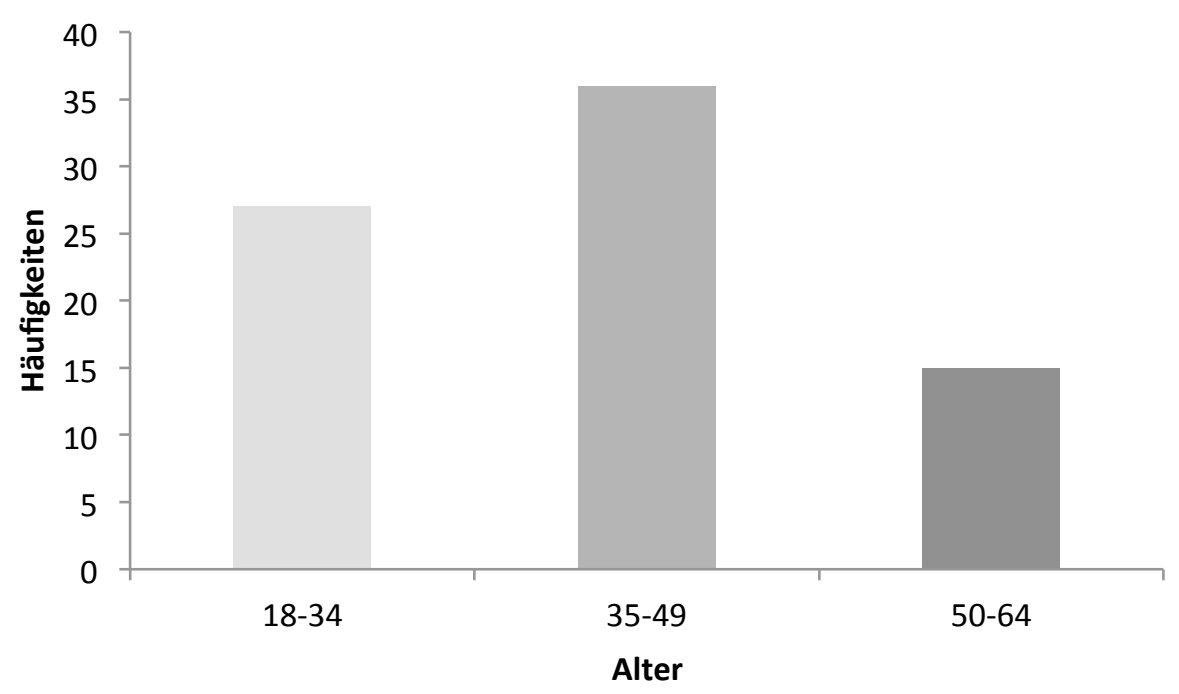

Abbildung 7. Häufigkeitsverteilung der Studienteilnehmer nach Altersgruppen in der Gesamtstichprobe $(N=78)$.

Soziodemographische Angaben. Insgesamt 68 (87.2\%) Probanden gaben Deutsch als ihre Muttersprache an, zehn Probanden (13.8\%) berichteten eine andere Muttersprache aus dem europäischen Sprachraum. Für alle Studienteilnehmer konnte ein ausreichend hohes Maß an deutschen Sprachkenntnissen festgestellt werden.

Insgesamt 41 (53.2\%) Probanden waren zum Zeitpunkt der Erhebung alleinstehend oder geschieden, 36 (46.8\%) in fester Partnerschaft oder verheiratet.

Insgesamt 56 (72.7\%) Probanden gaben an, mehr als 12 Jahre Schulausbildung absolviert zu haben. In der Gesamtstichprobe berichtete ein Studienteilnehmer keinen Schulabschluss zu haben, vier (5.2\%) einen Realschulabschluss, neun (11.7\%) Abitur, 17 (22.1\%) eine abgeschlossene Ausbildung, acht (10.4\%) Fachabitur, 31 (40.3\%) ein abgeschlossenes Hochschulstudium und sieben (9.1\%) eine abgeschlossene Promotion. Zum Zeitpunkt der Erhebung waren 46 (59.7\%) Probanden berufstätig, fünf (6.5\%) arbeitsuchend, neun (11.7\%) Studenten, zwei (2.6\%) in Ausbildung, sechs (7.8\%) Rentner, zwei (2.6\%) Hausfrauen/-männer, sechs (7.7\%) nicht berufstätig ohne weitere Angaben und ein Proband (1.3\%) gab dazu keine Auskunft. Die Verteilung der Berufsgruppen belief sich auf 47 (61.8\%) Angestellte, vier (5.3\%) Beamte, zwei (2.6\%) leitende Angestellte, acht (10.5\%) Freiberufler/Selbständige und 15 (19.7\%) sonstige Berufsgruppen, zwei (2.6\%) der Studienteilnehmer machten dazu keine Angaben. 
Insgesamt 37 (48.7\%) Studienteilnehmer gaben an, jemals Raucher gewesen zu sein, 22 (28.6\%) Studienteilnehmer waren Raucher zum Zeitpunkt der Datenerhebung. Der mittlere Body-Mass-Index lag bei 25.52 (SD = 5.20) in der Gesamtstichprobe. Eine Übersicht der soziodemographischen Daten findet sich in Tabelle 10.

Tabelle 10

Soziodemographische Angaben in der Gesamtstichprobe ( $N=78)$

\begin{tabular}{lll}
\hline Soziodemographische Angaben & & \\
\hline Alter, Mittelwert (SD) & 39.42 & $(10.32)$ \\
Deutsch als Muttersprache, $n$ (\%) & 68 & $(87.2)$ \\
Geschlecht, $n$ (\% weiblich) & 49 & $(62.8)$ \\
Geschlecht, $n$ (\% männlich) & 29 & $(37.2)$ \\
Familienstand, in fester Partnerschaft, $n$ (\%) & 36 & $(46.8)$ \\
Familienstand, alleinstehend oder geschieden, $n$ (\%) & 41 & $(53.2)$ \\
Bildung > 12 Jahre, $n$ (\%) & 56 & $(72.7)$ \\
Berufstätig aktuell, $n$ (\%) & 46 & $(59.7)$ \\
Berufsgruppe Angestellte, $n$ (\%) & 47 & $(61.8)$ \\
Body-Mass-Index, Mittelwert (SD) & 25.52 & $(5.20)$ \\
Raucher, aktuell, $n$ (\%) & 22 & $(28.6)$ \\
Raucher, jemals, $n$ (\%) & 37 & $(48.7)$ \\
\hline
\end{tabular}

Depressive Symptomatik aktuell. Der durchschnittliche Schweregrad der depressiven Symptomatik lag zum Zeitpunkt der Erhebung bei einem mittleren BDI-Summenwert von 14.46 Punkten $(S D=12.70)$. Die Teilnehmer wiesen eine depressive Symptomatik im Wertebereich zwischen null und 53 Punkten des BDI-Summenwertes auf. Insgesamt 29 (37.2\%) Studienteilnehmer berichteten einen BDI-Summenwert zwischen null und acht Punkten, wonach keine Depression vorlag. Bei 14 Studienteilnehmern (17.9\%) fand sich ein BDI-Summenwert zwischen neun und 13 Punkten, was einer minimalen Depression entspricht, bei neun (11.5\%) Studienteilnehmern lag ein BDI-Summenwert zwischen 14 und 19 Punkten vor, was einer leichten Depression entspricht. Bei 12 Studienteilnehmern fand 
sich ein BDI-Summenwert zwischen 20 und 28 Punkten, was einer mittelschweren Depression entspricht und bei 11 (14.1\%) Studienteilnehmern ergab sich ein BDISummenwert zwischen 29 und 60 Punkten, was einer schweren Depression entspricht. Eine Übersicht über die Häufigkeitsverteilung der BDI-Summenwerte in der Gesamtstichprobe findet sich in Abbildung 8. Bei insgesamt 52 (69.3\%) Studienteilnehmern lag der Summenwert des BDI unter einem Punktwert von 20.

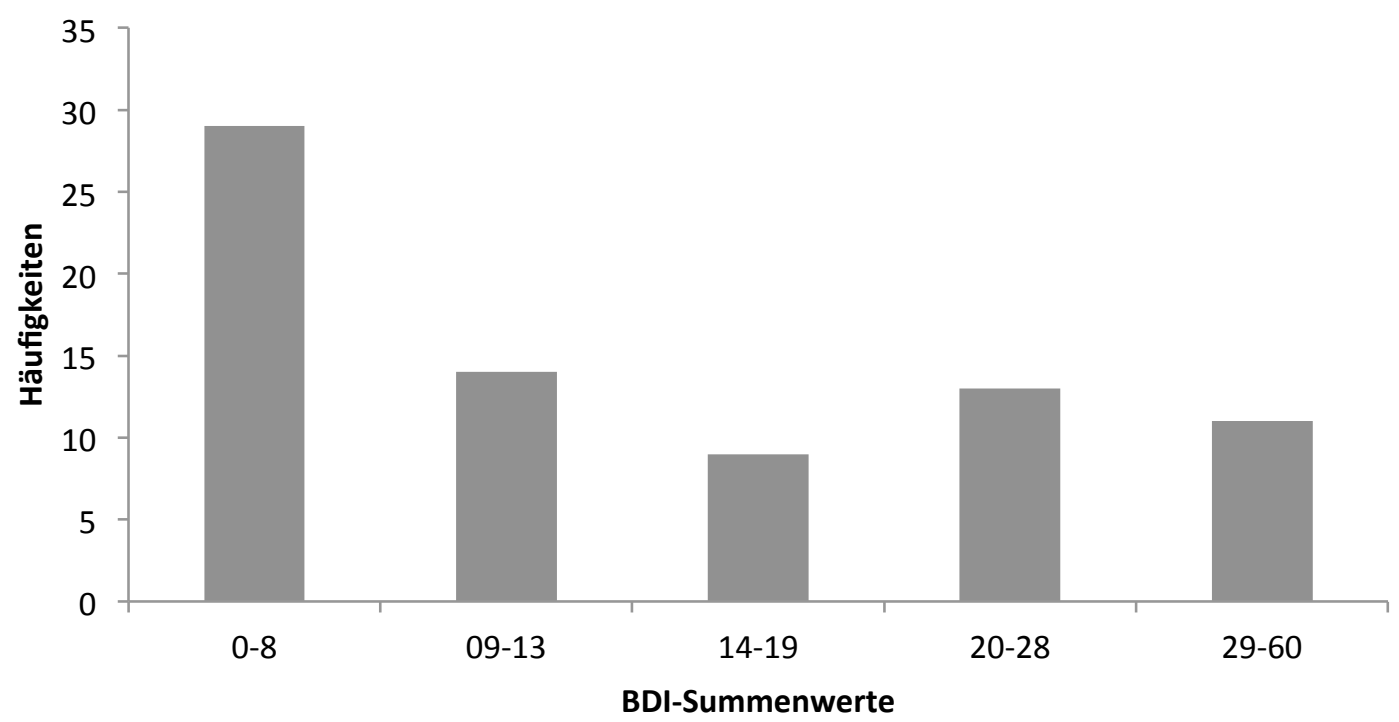

Abbildung 8. Häufigkeitsverteilung der BDI-Summenwerte in der Gesamtstichprobe $(N=78)$; Einteilung der Schweregrade nach Hautzinger et al. (2009): 0-8 Punkte $=$ keine Depression; 9-13 Punkt = minimale Depression; $14-19=$ leichte Depression; 20-28 mittelschwere Depression; 29-60 = schwere Depression.

\subsection{Ergebnisse der Fragestellungen}

Risikofaktoren für einen rezidivierenden Krankheitsverlauf. Insgesamt 24 (30.8\%) Studienteilnehmer wurden der Gruppe Erstmanifestation und 54 (69.2\%) Studienteilnehmer der Gruppe rezidivierender Verlauf zugeordnet. Acht (10.3\%) Männer und 16 (20.5\%) Frauen gaben an, im Lebensverlauf nur eine einzelne depressive Episode erlebt zu haben. 21 Männer (26.9\%) und 33 Frauen (42.3\%) berichteten einen rezidivierenden Verlauf der Erkrankung. 
Es zeigte sich kein signifikanter Unterschied in der Häufigkeitsverteilung von Männern und Frauen bezüglich des Krankheitsverlaufs. Die Häufigkeitsverteilungen für Männer und Frauen getrennt nach Krankheitsverlauf sind in Abbildung 9 dargestellt. Diejenigen Probanden, welche einen rezidivierenden Verlauf berichteten, unterschieden sich bezüglich des Lebensalters bei Datenerhebung nicht von den Probanden mit einer Erstmanifestation der Erkrankung. Rezidivierende Verläufe wiesen im Mittel $2.75(S D=1.69)$ depressive Episoden auf.

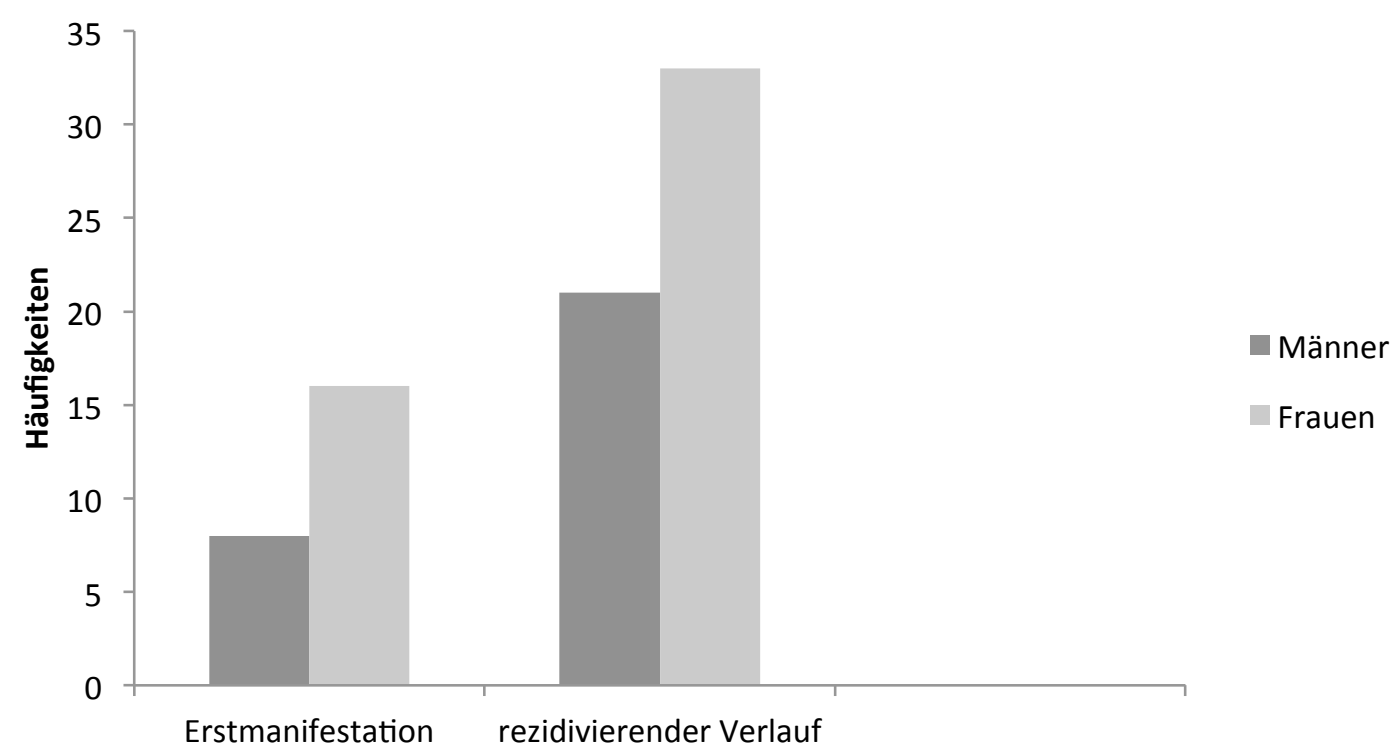

Abbildung 9. Häufigkeitsverteilung der Studienteilnehmer getrennt nach Geschlecht und Krankheitsverlauf (Erstmanifestation vs. rezidivierender Verlauf) in der Gesamtstichprobe $(N=78)$.

Die Stichprobenbeschreibung und die soziodemographischen Charakteristika getrennt nach den Gruppen Erstmanifestation und rezidivierender Verlauf sind in Tabelle 11 zur Übersicht dargestellt. 
Tabelle 11

Soziodemographische Angaben getrennt für den Krankheitsverlauf (Erstmanifestation vs. rezidivierender Verlauf) in der Gesamtstichprobe ( $N=78)$

\begin{tabular}{lcccc}
\hline Soziodemographische Angaben & \multicolumn{2}{c}{ Erstmanifestation } & \multicolumn{2}{c}{ Rezidivierender Verlauf } \\
\hline & $M$ & $S D$ & & $S D$ \\
\hline Alter & 36.88 & 9.15 & 40.56 & 10.69 \\
Body-Mass-Index & 24.77 & 4.15 & 25.86 & 5.61 \\
\hline & $N$ & $\%$ & $N$ & $\%$ \\
\hline Geschlecht, weiblich & 16 & 66.7 & 33 & 61.1 \\
Geschlecht, männlich & 8 & 33.3 & 21 & 38.9 \\
In fester Partnerschaft & 15 & 62.5 & 21 & 39.7 \\
Alleinstehend oder geschieden & 9 & 37.5 & 32 & 59.3 \\
Bildung > 12 Jahre & 17 & 70.8 & 39 & 73.6 \\
Berufstätig aktuell & 18 & 75.0 & 28 & 51.9 \\
Berufsgruppe Angestellte & 14 & 58.3 & 33 & 61.1 \\
\hline
\end{tabular}

Psychiatrische Erkrankungen in der Familie. Eine Darstellung der Häufigkeiten der psychiatrischen Erkrankungen in der Familie getrennt für die Gruppen Erstmanifestation und rezidivierender Verlauf gibt Tabelle 12. Psychiatrische Erkrankungen in der Familie im Allgemeinen wurden von 17 (70.8\%) Studienteilnehmern aus der Gruppe der Erstmanifestation und 41 (75.9\%) Teilnehmern aus der Gruppe der rezidivierenden Verläufe berichtet. Depressive Erkrankungen in der Primärfamilie fanden sich bei neun (37.5\%) Probanden aus der Gruppe der Erstmanifestation und 31 (57.4\%) Probanden aus der Gruppe der rezidivierenden Verläufe. Suizid bei Angehörigen wurde in beiden Gruppen von knapp 30\% der Probanden berichtet. 
Tabelle 12

Deskriptive Statistik psychiatrischer Erkrankungen innerhalb der Familie getrennt für den Krankheitsverlauf (Erstmanifestation vs. rezidivierender Verlauf) in der Gesamtstichprobe $(N=78)$

\begin{tabular}{lcccc}
\hline & \multicolumn{2}{c}{ Erstmanifestation } & \multicolumn{2}{c}{ Rezidivierender Verlauf } \\
\hline & $N$ & $\%$ & \multirow{2}{*}{$\%$} & 75.9 \\
\hline $\begin{array}{l}\text { Psychische Erkrankungen in der } \\
\text { Familie allgemein }\end{array}$ & 17 & 70.8 & 31 & 57.4 \\
$\begin{array}{l}\text { Depressive Erkrankungen in der } \\
\text { Primärfamilie }\end{array}$ & 9 & 37.5 & & 27.8 \\
Suizid bei Angehörigen & 7 & 29.2 & 15 & \\
\hline
\end{tabular}

Chi-Quadrat-Tests zeigten keinen statistisch signifikanten Unterschied in der familiären Häufung psychiatrischer Erkrankungen zwischen Probanden mit einem rezidivierenden Krankheitsverlauf und einer Erstmanifestation in der vorliegenden Stichprobe zum Zeitpunkt der Erhebung. Das Vorliegen einer psychiatrischen Erkrankung hatte ebenso wie das spezifische Vorliegen einer Depression innerhalb der Familie keinen statistisch signifikanten Einfluss auf die Anzahl der Episoden bei rezidivierenden depressiven Störungen. Es konnte ebenso kein Unterschied in der Häufigkeitsverteilung familiärer Suizide für Probanden mit rezidivierenden Verläufen und Probanden mit Erstmanifestation und kein Einfluss auf die Anzahl der Episoden festgestellt werden.

Probanden mit einer positiven Familiengeschichte für depressive Erkrankungen in der Primärfamilie hatten ein durchschnittliches Ersterkrankungsalter von 25 Jahren $(S D=10.43)$. Probanden ohne depressive Erkrankungen in der Primärfamilie wiesen ein Ersterkrankungsalter von 30 Jahren auf $(S D=12.56)$. Der Unterschied war statistisch nicht signifikant.

Familienstand. Insgesamt neun (37.5\%) der Probanden aus der Gruppe der Erstmanifestationen und 32 (59.3\%) der Probanden mit rezidivierendem Krankheitsverlauf waren alleinstehend oder geschieden zum Zeitpunkt der Datenerhebung. Es ergab sich kein signifikanter Unterschied in der Häufigkeitsverteilung der alleinstehenden und in fester Partnerschaft lebenden Probanden bezüglich des Krankheitsverlaufs. Die Analysen ergaben 
einen marginal signifikanten Unterschied in der retrospektiv berichteten Episodenanzahl für Personen in festen Partnerschaften und aktuell alleinstehenden Probanden, $t(73)=-1.95, p=$ $.055, d=.45$. Alleinstehende Personen wiesen dabei marginal signifikant mehr depressive Episoden über den Lebensverlauf auf $(M=3.10, S D=1.62)$ als Personen in aktuell festen Partnerschaften $(M=2.36, S D=1.69)$.

Ersterkrankungsalter. Das Ersterkrankungsalter einer depressiven Episode lag in der Gesamtstichprobe im Mittel bei 27.25 Jahren $(S D=10.80)$. Der Median für das Ersterkrankungsalter lag bei 26 Jahren (IQR = 18). Der Wertebereich des Ersterkrankungsalters reichte von sechs bis 48 Jahre. Es gab keinen statistisch signifikanten Unterschied im Ersterkrankungsalter zwischen Männer und Frauen.

Univariate Varianzanalysen ergaben signifikante Haupteffekte für die Faktoren Krankheitsverlauf $F(2,70)=24.68, p<.001, f=.59$ und Altersgruppe, $F(2,70)=21.80, p<$ $.001, f=.79$ bezüglich des Ersterkrankungsalters in der Gesamtstichprobe. Es gab keine statistisch signifikante Interaktion zwischen den Faktoren Krankheitsverlauf und Altersgruppe bezüglich des Ersterkrankungsalters.

Simple-Effect-Kontraste zeigten, dass Probanden mit rezidivierenden Verläufen der Erkrankung in allen Altersgruppen ein signifikant niedrigeres Ersterkrankungsalter berichteten als Probanden aus der Gruppe der Erstmanifestationen (alle $p$ 's $<.01$ ). Lediglich in der Altersgruppe der 50-63-Jährigen war der Unterschied nur marginal signifikant ( $p=$ .054). Die Mittelwerte und Standardabweichungen der einzelnen Kategorien finden sich in Tabelle 13. 
Tabelle 13

Mittelwerte und Standardabweichungen des Ersterkrankungsalters getrennt für Krankheitsverlauf und Altersgruppe in der Gesamtstichprobe $(N=78)$

\begin{tabular}{llll}
\hline Kategorie & & $M$ & $S D$ \\
\hline $18-34$ & Einzelne Episode & 25.58 & 2.24 \\
& Rezidivierender Verlauf & 16.43 & 2.07 \\
$35-49$ & Einzelne Episode & 39.70 & 2.45 \\
& Rezidivierender Verlauf & 24.48 & 1.55 \\
$50-63$ & Einzelne Episode & 45.00 & 5.48 \\
& Rezidivierender Verlauf & 33.46 & 2.15 \\
\hline
\end{tabular}

Paarweise Vergleiche zeigten, dass sich das Ersterkrankungsalter im Rahmen eines rezidivierenden Verlaufs für alle drei Altersgruppen statistisch signifikant unterschied (jeweils $p<.01$ ). Das niedrigste Ersterkrankungsalter fand sich in der Altersgruppe der 18-34Jährigen $(M=16.43, S D=2.07)$, gefolgt von der Altersgruppe der 35-49-Jährigen $(M=24.48$, $S D=1.55)$. Das höchste Ersterkrankungsalter gaben Probanden der Altersgruppe der 50-63Jährigen $(M=33.46, S D=2.15)$ an. Probanden aus der Gruppe mit Erstmanifestation, d.h. mit einer einzelnen Episode einer depressiven Erkrankung im bisherigen Lebensverlauf zeigten den gleichen Trend (jeweils $p<.01$ ), jedoch gab es hier keinen statistisch signifikanten Unterschied zwischen der Altersgruppe der 35-49 ( $M=39.70, S D=2.45)$ und 50-63-Jährigen $(M=45.00, S D=5.48)$ bezüglich des Ersterkrankungsalters. Abbildung 10 zeigt die Haupteffekte für das Ersterkrankungsalter getrennt nach Krankheitsverlauf und Altersgruppe. 


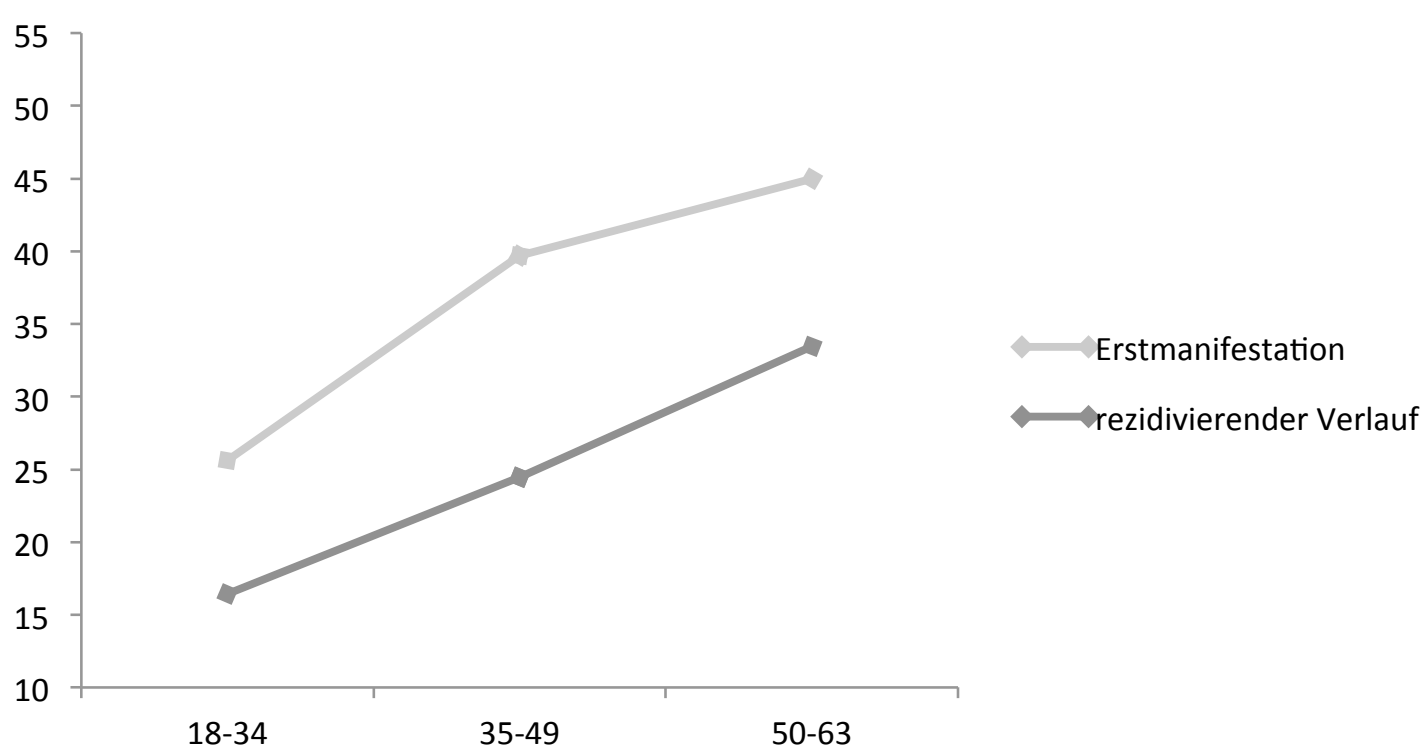

Abbildung 10. Ersterkrankungsalter nach Altersgruppe und Krankheitsverlauf in der Gesamtstichprobe ( $N=78$ ); Einteilung der Altersgruppen nach (Kessler et al., 2010). Haupteffekte für Altersgruppe und Krankheitsverlauf $(\mathrm{p}<.001)$

In linearen Regressionsanalysen war das Ersterkrankungsalter zudem ein statistisch signifikanter Prädiktor für die Anzahl der retrospektiv berichteten depressiven Episoden im Lebensverlauf, $B=-.36, p<.001, R^{2}=.13$. Auch nach Kontrolle der Faktoren Geschlecht und Alter war das Ersterkrankungsalter ein statistisch signifikanter Prädiktor für die Anzahl der retrospektiv berichteten depressiven Episoden im Lebensverlauf $(p<.001)$. Je niedriger das Ersterkrankungsalter war, desto mehr depressive Episoden berichteten die Studienteilnehmer retrospektiv über den Lebensverlauf hinweg.

Ersterkrankungsalter bei diagnostizierten Episoden und nicht diagnostizierten Episoden. Der Mittelwert des Ersterkrankungsalters für ausschließlich diagnostizierte ErstEpisoden lag im Durchschnitt bei 29.88 Jahren $(S D=10.53)$. Der Mittelwert des Ersterkrankungsalters für nicht diagnostizierte Episoden lag bei 21.48 Jahren $(S D=9.40)$. Das Ersterkrankungsalter lag damit für diagnostizierte Episoden deutlich höher als für subjektiv berichtete und nicht diagnostizierte Episoden. Der Unterschied zwischen den Gruppen war signifikant, $t(73)=-3.29, p<.01, d=1.23$. Die beiden Gruppen unterschieden sich bei Datenerhebung nicht bezüglich ihres aktuellen Lebensalters oder des Geschlechts. In der Gruppe der Probanden mit rezidivierendem Krankheitsverlauf zeigte sich jedoch kein 
Unterschied zwischen den Probanden mit diagnostizierten Erstepisoden und nichtdiagnostizierter Erstepisoden bezüglich der Anzahl der Episoden im weiteren Lebensverlauf $(p=.62)$.

Neurotizismus und Extraversion. In unabhängigen t-Tests zeigte sich ein marginal signifikanter Unterschied in der Ausprägung der Persönlichkeitsvariable Neurotizismus für Probanden mit einer Erstmanifestation einer depressiven Erkrankung und Probanden mit einem rezidivierenden Verlauf, $t(55)=-1.85, p=.07$. Dabei wiesen Probanden mit rezidivierenden Verläufen der Erkrankung im Mittel höhere Neurotizismuswerte auf $(M=$ 25.32, $S D=6.39$ ) als Probanden welche eine Erstmanifestation einer depressiven Erkrankung berichteten $(M=21.19, S D=7.98)$. Die Ausprägung auf der Skala Neurotizismus war zudem ein statistisch signifikanter Prädiktor für das Ersterkrankungsalter, $B=-.30, p<.05, R^{2}=.08$, aber kein statistisch signifikanter Prädiktor für die Anzahl der Episoden im bisherigen Lebensverlauf. Die Neurotizismusausprägung stellte auch nach Kontrolle des Geschlechts einen statistisch signifikanten Prädiktor für das Ersterkrankungsalter dar, $b=-.29, p<.05, R^{2}$ $=.10$. Je höher die Ausprägung auf der Skala Neurotizismus war, desto früher lag das retrospektiv berichtete Ersterkrankungsalter der Probanden in der vorliegenden Stichprobe.

In weiteren unabhängigen t-Tests zeigte sich auch ein marginal signifikanter Unterschied in der Ausprägung der Persönlichkeitsvariable Extraversion für Probanden bei Erstmanifestation und Probanden mit einem rezidivierenden Verlauf, $t(55)=1.72, p=.09$. Dabei wiesen Probanden mit rezidivierenden Verläufen der Erkrankung im Mittel niedrigere Extraversionswerte auf $(M=19.71, S D=6.80)$ als Probanden welche eine Erstmanifestation berichteten $(M=23.16, S D=6.86)$. Die Ausprägung auf der Skala Extraversion stellte ebenfalls einen statistisch signifikanten Prädiktor für das Ersterkrankungsalter dar, $B=.29, p$ $<.05, R^{2}=.08$. Die Extraversionausprägung stellte auch nach Kontrolle des Geschlechts einen statistisch signifikanten Prädiktor für das Ersterkrankungsalter dar, $B=.07, p<.05, R^{2}=.09$. Je höher die Ausprägung der Probanden auf der Skala Extraversion war, desto später lag das retrospektiv berichtete Ersterkrankungsalter der Probanden in der vorliegenden Stichprobe. Auch hier gab es jedoch keinen Zusammenhang mit der Anzahl der depressiven Episoden im bisherigen Lebensverlauf. Die Mittelwerte und marginal signifikanten Unterschiede für die Ausprägungen auf den Skalen Neurotizismus und Extraversion für den Faktor Krankheitsverlauf sind in Abbildung 11 dargestellt. 


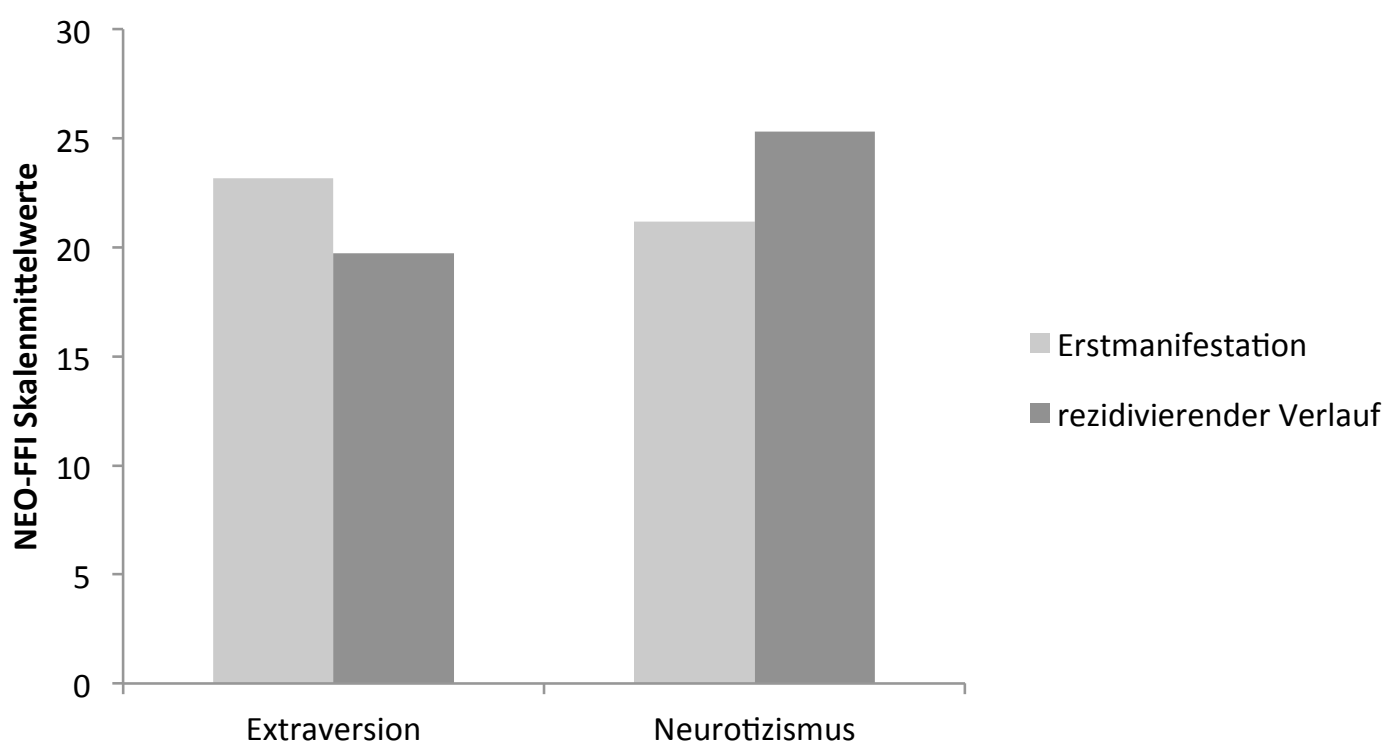

Abbildung 11. Marginal signifikanter Unterschied in den Persönlichkeitsausprägungen Extraversion ( $p$ $=.09$ ) und Neurotizismus ( $p=.07$ ) für den Faktor Krankheitsverlauf in der Gesamtstichprobe $(N=78)$.

Frühkindliche Traumata. In multivariaten Analysen zeigten sich in der vorliegenden Stichprobe kein Zusammenhang zwischen dem Krankheitsverlauf und traumatischen Erfahrungen in der Kindheit und ebenso kein Zusammenhang zwischen der Anzahl der berichteten Episoden mit traumatischen Erfahrungen in der Kindheit. Die Probanden mit einem rezidivierenden Verlauf wiesen keine höheren Ausprägungen auf den Skalen des CTQ auf, als Probanden bei Erstmanifestation einer depressiven Erkrankung.

Dauer der Prodromalphase. In der Gesamtstichprobe berichteten insgesamt 73 (93.6\%) Studienteilnehmer eine Prodromalphase erlebt $\mathrm{zu}$ haben, davon 51 (69.9\%) Studienteilnehmer eine Phase psychopathologischer Veränderungen von mehr als einem Monat vor Beginn der voll ausgeprägten Symptome und 22 (30.1\%) Studienteilnehmer eine Prodromalphasendauer von weniger als einem Monat. Nur fünf Probanden konnten keine zeitlichen oder inhaltlichen Angaben zur Prodromalphase machen. Die Studienteilnehmer berichteten für die letzte Episode der depressiven Erkrankung im Durchschnitt eine Prodromalphasendauer von 9.40 Monaten $(S D=15.49)$. Insgesamt zehn Probanden beschrieben eine Prodromalphasendauer von mehr als 23 Monaten. Im Rahmen univariater 
Varianzanalysen ergab sich für die Gesamtstichprobe ein Haupteffekt für das Geschlecht bezüglich der Prodromalphasendauer $F(3,69)=4.59, p<.05, f=.26$. Die Männer wiesen hier im Mittel eine längere Prodromalphasendauer in Monaten auf $(M=16.20, S D=3.32)$ als die Frauen $(M=7.50, S D=3.33)$. Es ergab sich weiterhin ein marginal signifikanter Haupteffekt für den Krankheitsverlauf bezüglich der Prodromalphasendauer, $F(3,69)=3.29, p=.074, f=$ .22. Die durchschnittliche Prodromalphasendauer war im Rahmen einer Erstmanifestation mit 15.53 Monaten $(S D=3.42)$ länger als im Rahmen eines Rezidivs mit durchschnittlich 8.17 Monaten $(S D=2.18)$. Die Abbildung 12 zeigt den signifikanten Haupteffekt für das Geschlecht in der Gruppe der Erstmanifestation und den marginal signifikanten Haupteffekt für den Krankheitsverlauf.

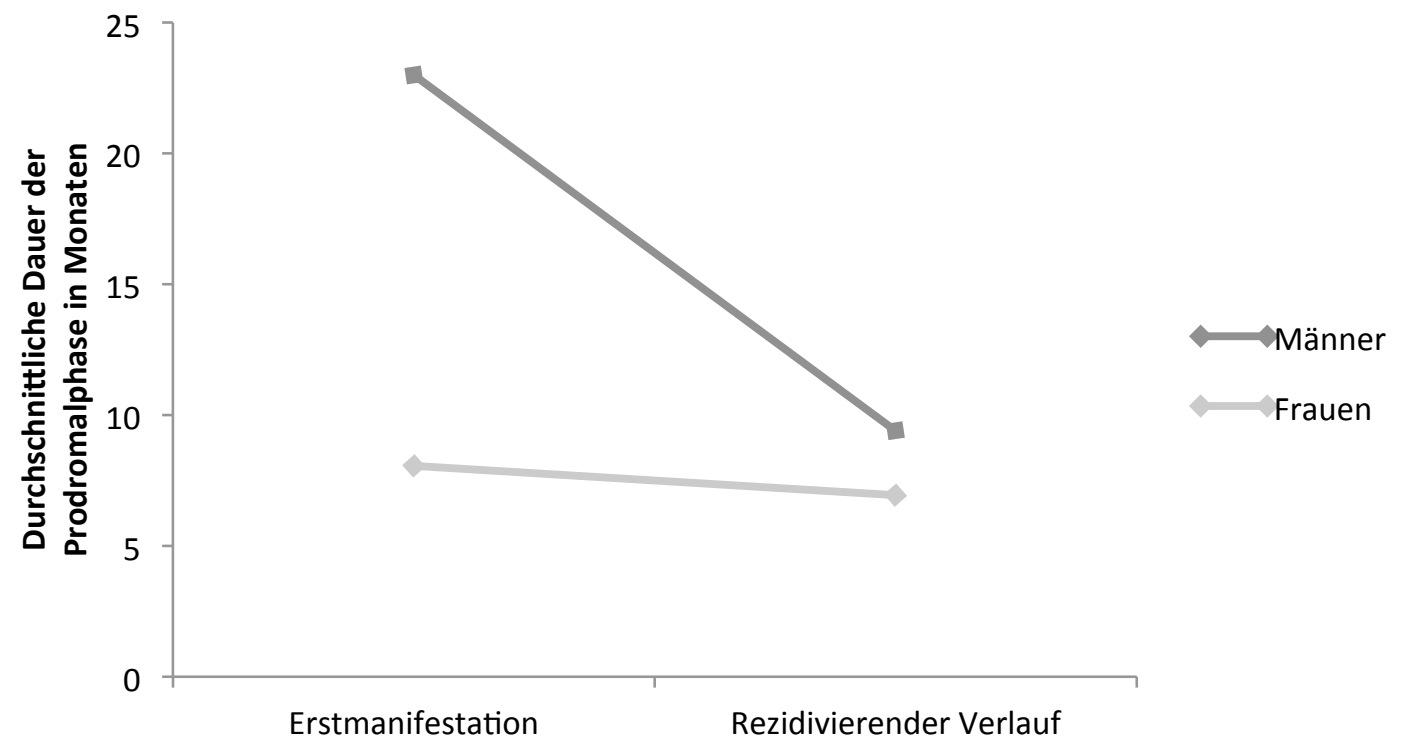

Abbildung 12. Durchschnittliche Dauer der Prodromalphase getrennt für Männer und Frauen sowie den Krankheitsverlauf (Erstmanifestation vs. rezidivierender Verlauf) in der Gesamtstichprobe ( $N=$ 78).

Ein Interaktionseffekt zwischen Geschlecht und Krankheitsverlauf konnte in der vorliegenden Stichprobe nicht gezeigt werden. Es ergab sich weiterhin ein margnial signifikanter Haupteffekt für die Altersgruppe, $F(2,70)=2.50, p=.090, f=.27$ in der Gesamtstichprobe. Dabei wiesen Probanden aus der Altersgruppe der 18-34-Jährigen die höchste Prodromalphasendauer auf $(M=14.73, S D=19,27)$, gefolgt von den Probanden der 
Altersgruppe der 35-49-Jährigen $(M=6.56, S D=12.92)$ und den Probanden der Altersgruppe der 50-63-Jährigen $(M=6.15, S D=10.52)$. Post-hoc-Analysen ergaben keine statistisch signifikanten Unterschiede zwischen den Altersgruppen.

Simple-Effect-Analysen ergaben, dass es nur im Rahmen einer Erstmanifestation einen statistisch signifikanten Effekt für das Geschlecht gab $(p=.033)$. Nur in der Gruppe der Erstmanifestation wiesen Männer eine statistisch signifikant längere Prodromalphasendauer auf als Frauen, nicht jedoch in der Gruppe der rezidivierenden Verläufe. Die Tabelle 14 gibt eine Übersicht über die Mittelwerte und Standardabweichungen in den einzelnen Kategorien der Simple-Effect-Kontraste. Der Unterschied in der Prodromalphasendauer zwischen Erstmanifestation und Rezidiv war lediglich in der Gruppe der Männer signifikant ( $p$ $=$.044). Frauen zeigten keinen statistisch signifikanten Unterschied in der Prodromalphasendauer bei einer Erstmanifestation und einem Rezidiv.

Tabelle 14

Mittelwerte und Standardabweichungen der Prodromalphasendauer in Monaten für die Kategorien Geschlecht und Krankheitsverlauf in der Gesamtstichprobe $(N=78)$

\begin{tabular}{lll}
\hline Kategorie & $M$ & $S D$ \\
\hline Männer-Erstmanifestation & 23.00 & 5.71 \\
Männer-Rezidiv & 9.40 & 3.38 \\
Frauen-Erstmanifestation & 8.06 & 3.78 \\
Frauen-Rezidiv & 6.93 & 2.76 \\
\hline
\end{tabular}

In der Abbildung 13 sind die Mittelwerte der durchschnittlichen Prodromalphasendauer für die Altersgruppe der 18-34-Jährigen, getrennt nach Geschlecht und Krankheitsverlauf dargestellt. Simple-Effect-Kontraste zeigten hier keine statistisch signifikanten Unterschiede in der Prodromalphasendauer zwischen Männern und Frauen und zwischen Probanden mit rezidivierendem Krankheitsverlauf und Erstmanifestation. 


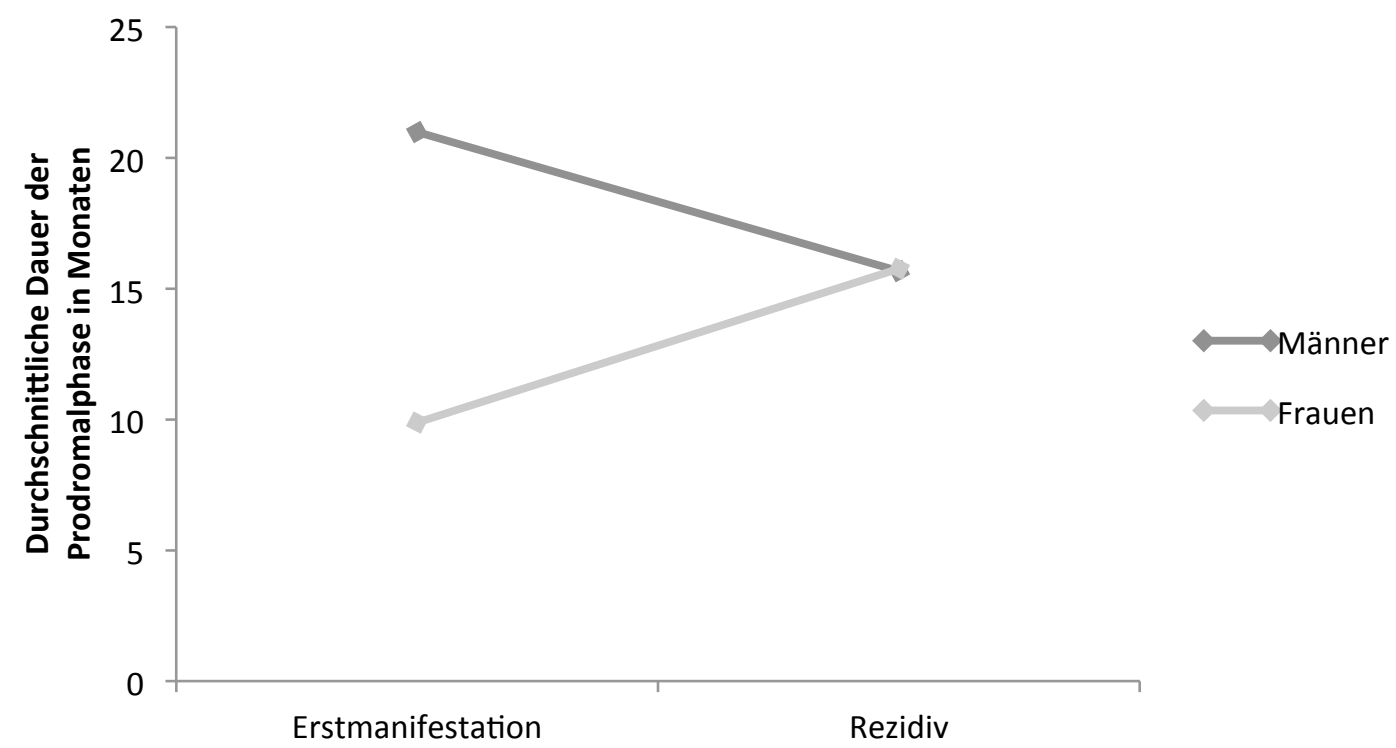

Abbildung 13. Durchschnittliche Dauer der Prodromalphase in Monaten für die Altersgruppe der 1834-Jährigen getrennt nach Krankheitsverlauf und Geschlecht. Keine statstisch signifikanten Effekte.

In der Altersgruppe der 35-49-Jährigen konnten in weiteren univarianten Analysen ein marginal signifikanter Haupteffekt bezüglich des Krankheitsverlaufs, $F(3,30)=6.49, p=$ $.07, f=.22$, als auch ein statistisch signifikanter Haupteffekt für das Geschlecht gefunden werden, $F(3,30)=5.23, p<.05, f=.26$. Männer zeigten dabei in der Gruppe der Erstmanifestationen mit 25.67 Monaten $(S D=39.31$ ) die längste Prodromalphasendauer im Vergleich zu Männern in der Gruppe der rezidivierenden Verläufe $(M=5.92, S D=6.91)$ und im Vergleich zu Frauen aus der Gruppe der Erstmanifestationen $(M=7.17, S D=9.97)$ und Frauen aus der Gruppe der rezidivierenden Verläufe $(M=2.46, S D=2.50)$. Eine statistisch signifikante Interaktion lag nicht vor. Simple-Effect-Kontraste zeigten, dass der Unterschied zwischen Männern und Frauen lediglich in der Gruppe der Erstmanifestationen statistisch signifikant war $(p<.05)$ und es auch nur in der Gruppe der Männer einen Unteschied in der Dauer der Prodromalphase zwischen den Probanden bei Erstmanifestation und den Probanden mit rezidivierenden Verläufen gab $(p<.05)$. Die Haupteffekte sind in Abbildung 14 dargestellt. 


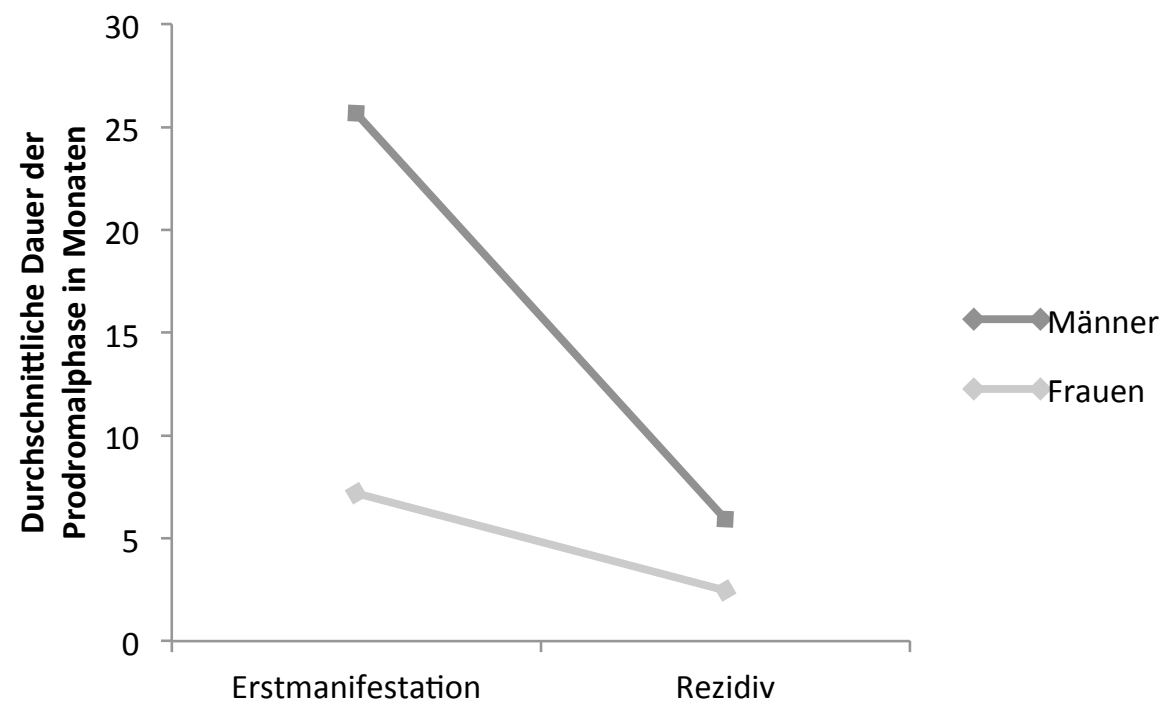

Abbildung 14. Durchschnittliche Dauer der Prodromalphase in Monaten für die Altersgruppe der $35-$ 49-Jährigen getrennt nach Krankheitsverlauf und Geschlecht. Marginal signifikanter Haupteffekt für den Krankheitsverlauf $(p=.07)$ und signifikanter Haupteffekt für Geschlecht $(p<.05)$.

Für die Altersgruppe der 50-63-Jährigen konnten keine statistisch signifikanten Hauptoder Interaktionseffekte bezüglich der Dauer der Prodromalphase gefunden werden. Die Mittelwerte der Prodromalphasendauer getrennt für Männer und Frauen und den Krankheitsverlauf sind für die Altersgruppe der 50-63-Jährigen in Abbildung 15 dargestellt.

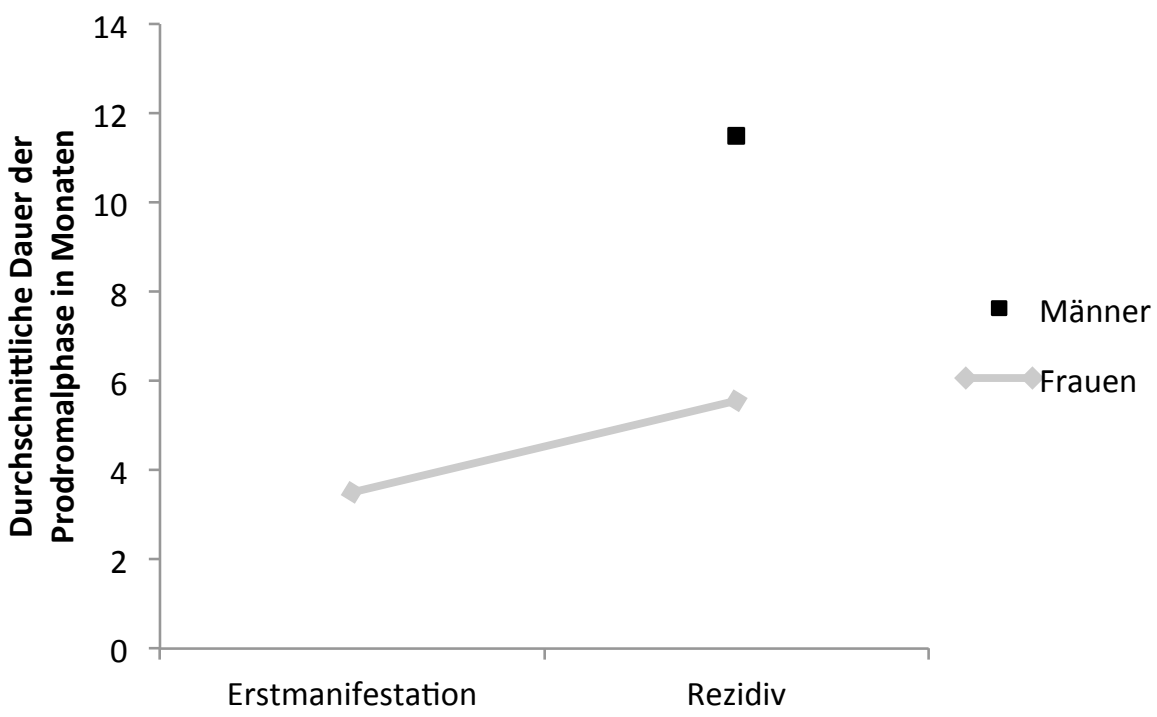

Abbildung 15. Durchschnittliche Dauer der Prodromalphase in Monaten für die Altersgruppe der 5063-Jährigen getrennt nach Krankheitsverlauf und Geschlecht. Fehlende Zellbesetzung für Männer Erstmanifestation. Keine statistisch signifikanten Effekte. 
Zwischen der Dauer der Prodromalphase und der Anzahl der retrospektiv berichteten Episoden im bisherigen Lebensverlauf gab es keinen statistisch signifikanten Zusammenhang. Es konnte ebenfalls kein signifikanter Unterschied in der Dauer der berichteten Prodromalphase für Probanden mit einem BDI-II Summenwert $<20$ und einem BDI-II Summenwert $\geq 20$ festgestellt werden.

Symptome der Prodromalphase. Die qualitative Auswertung der berichteten Prodromalsymptome und die erfolgte Kategorienbildung sowie deren Häufigkeitszuteilungen finden sich in Tabelle 15. Am häufigsten wurden somatische Beschwerden (64.1\%), Schlafstörungen (42.3\%) und Ängste/Sorgen (26.9\%) berichtet. Männer und Frauen unterschieden sich in der Häufigkeit der berichteten Emotionalen Labilität und Empfindsamkeit signifikant, $\chi^{2}(1)=5.10, p<.05$. Dies spricht dafür, dass die Chance für Frauen 7.25-mal höher war, Symptome emotionaler Labilität und Empfindsamkeit für die Prodromalphase zu berichten als für Männer.

Ebenso zeigte sich ein Geschlechterunterschied bei sonstigen körperlichen Missempfindungen, $\chi^{2}(1)=4.65, p<.05$. Dies spricht dafür, dass die Chance für Frauen 3.29mal höher war, Symptome von sonstigen körperlichen Missempfindungen für die Prodromalphase zu berichten als für Männer. Es ergaben sich keine weiteren signifikanten Unterschiede für andere berichtete Prodromalsymptome zwischen Männern und Frauen. Es konnten keine signifikanten Unterschiede in den Häufigkeitsverteilungen der Prodromalsymptome bezüglich des Krankheitsverlaufs (Erstmanifestation vs. Rezidiv) festgestellt werden. 
Tabelle 15

Häufigkeiten der Prodromalsymptome der letzten depressiven Episode in der Gesamtstichprobe ( $N=78)$

\begin{tabular}{|c|c|c|}
\hline Psychische Prodromalsymptome & $N$ & $\%$ \\
\hline Ängste und Sorgen & 21 & 26.9 \\
\hline Traurigkeit/gedrückte Stimmung & 20 & 25.6 \\
\hline Antriebs- und Energieminderung/Lustlosigkeit & 16 & 20.5 \\
\hline Grübeln & 13 & 16.7 \\
\hline Emotionale Labilität und Empfindsamkeit & 12 & 15.4 \\
\hline Sozialer Rückzug & 12 & 15.4 \\
\hline Konzentrationsstörungen & 12 & 15.4 \\
\hline Reizbarkeit & 11 & 14.1 \\
\hline Überforderungsgefühle & 9 & 11.5 \\
\hline Nervosität/Unruhe & 8 & 10.3 \\
\hline Freud- oder Interessenverlust & 8 & 10.3 \\
\hline Aggressivität/Wut & 5 & 6.4 \\
\hline Selbstwertgefühl reduziert & 4 & 5.1 \\
\hline Schuldgefühle/Selbstvorwürfe & 4 & 5.1 \\
\hline Symptome von Zwang oder Wahn & 4 & 5.1 \\
\hline Dysfunktionale Gedanken & 2 & 2.6 \\
\hline Körperliche Prodromalsymptome & $\mathrm{N}$ & $\%$ \\
\hline Somatische Beschwerden & 50 & 64.1 \\
\hline - Schmerzen & 14 & 17.9 \\
\hline - Magen-Darm Beschwerden & 11 & 14.1 \\
\hline - $\quad$ Sonstige körperliche Missempfindungen & 25 & 32.1 \\
\hline Schlafstörungen & 33 & 42.3 \\
\hline Erschöpfungsgefühl/Müdigkeit & 16 & 20.5 \\
\hline Veränderter Appetit & 13 & 16.7 \\
\hline Vermehrter Substanzgebrauch & 5 & 6.4 \\
\hline Anspannung & 5 & 6.4 \\
\hline Libidoverlust & 2 & 2.6 \\
\hline
\end{tabular}


Es gab weiterhin einen signifikanten Zusammenhang zwischen der Altersgruppe und dem Bericht von Appetitveränderungen in der Prodromalphase. Eine loglineare Analyse ergab ein Modell, welches alle Effekte beinhaltete. Der Likelihood Ratio dieses Modells war $\chi^{2}(0)=0, p=1$. Dies weist auf die statistische Signifikanz der Interaktion höchster Ordnung im Modell hin (Altersgruppe $x$ Bericht von Appetitveränderungen), $\chi^{2}(2)=6.14, p<.05$. Um diesen Effekt aufzubrechen wurden einzelne Chi-Quadrat Tests für die Altersgruppen durchgeführt. In der Gesamtstichprobe zeigte sich ein statistisch signifikanter Unterschied zwischen der ältesten und jüngsten Altersgruppe für das Berichten von Appetitveränderungen in der Prodromalphase, $\chi^{2}(1)=4.78, p<.05$. Der Effekt zeigte sich auch zwischen der ältesten und der mittleren Altersgruppe, $\chi^{2}(1)=5.61, p<.05$. Die Odds Ratios weisen darauf hin, dass die Chance Appetitveränderungen in der Prodromalphase zu berichten in der Altersgruppe der 50-64-Jährigen 5.36-mal höher war als in der Altersgruppe der 18-34-Jährigen und auch 5.36-mal höher als in der Altersgruppe der 35-49-Jährigen. In der Altersgruppe der 50-64-Jährigen kamen in der vorliegenden Stichprobe demnach Berichte von Appetitveränderungen in der Prodromalphase häufiger vor als bei den jüngeren Studienteilnehmern. Die Abbildung 16 zeigt die prozentualen Anteile der berichteten Appetitveränderungen über die einzelnen Altersgruppen. 


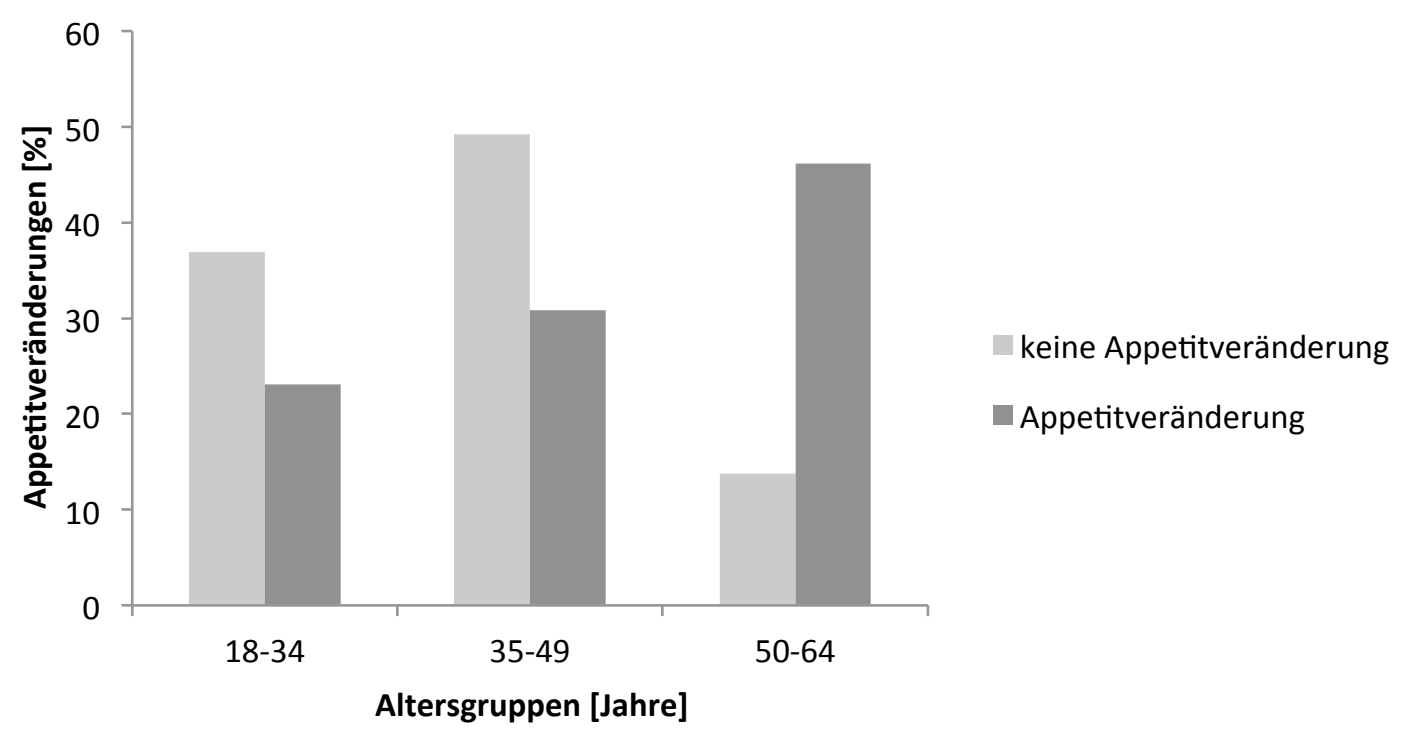

Abbildung 16. Prozentualer Anteil der berichteten Appetitveränderungen in den Altersgruppen der Gesamtstichprobe $(N=78)$.

Mittels weiteren Chi-Quadrat Tests konnten marginal signifikante Unterschiede in den berichteten Häufigkeiten für die Prodromalsymptome Appetitveränderungen, $\chi^{2}(1)=$ 3.35, $p=.09$ und sonstige körperliche Missempfindungen, $\chi^{2}(1)=3.82, p=.06$ für Probanden mit einem BDI-II Summenwert $<20$ und einem BDI-II Summenwert $\geq 20$ festgestellt werden. Dies spricht dafür, dass die Chance für Probanden mit leichter oder keiner akut depressiven Symptomatik 5.90-mal höher war, Symptome veränderten Appetits retrospektiv für die Prodromalphase zu berichteten. Die Chance körperliche Missempfindungen retrospektiv für die Prodromalphase zu berichten lag für die Probanden mit akut mittelgradiger oder schwerer depressiver Symptomatik 2.75-mal höher als für Probanden, die keine oder nur leichte depressive Symptome aufwiesen.

In weiterführenden Analysen konnte allerdings zwischen Probanden mit einem hohen BDI-Summenwert kein Unterschied zu Probanden mit einem niedrigen BDI-Summenwert bezüglich aktueller somatischer Beschwerden festgestellt werden. Bezüglich des Appetits ergab sich ein signifikanter Unterschied zwischen den Gruppen, $t(73)=-4.59, p<.001, d=$ 1.06. Probanden mit einem BDI-Summenwert $\geq 20$ wiesen aktuell stärkere Apptetitveränderungen auf $(M=1.48, S D=1.04)$ als Probanden mit einem BDI-Summenwert $<20(M=.54, S D=.70)$. Es gab allerdings keine signifikante Korrelation zwischen dem 
aktuellen Bericht von Appetitveränderungen und berichteten Appetitveränderungen in der Prodromalphase. Für alle anderen Prodromalsymptome fanden sich in der vorliegenden Stichprobe keine statistisch signifikanten Unterschiede in den berichteten Häufigkeiten bei Probanden mit akut mittelgradiger bis schwerer depressiver Symptomatik und Probanden ohne bis leichte depressive Symptomatik. Weder zwischen den Altersgruppen noch zwischen Männern und Frauen zeigten sich signifikante Unterschiede in der akuten depressiven Symptomatik.

Dauer der unbehandelten Episode (DUI, DUE). Die Dauer der unbehandelten Erkrankung (DUI) bzw. Episode (DUE) lag im Schnitt in der Gesamtstichprobe bei $M=5.78$ Monaten (SD = 14.74). Es ergab sich kein statistisch signifikanter Unterschied zwischen Probanden mit einem rezidivierenden Krankheitsverlauf und Probanden mit einer Erstmanifestation bezüglich der DUI/DUE der letzten depressiven Episode. Nur 54.2\% der Studienteilnehmer kontaktierten im Rahmen einer Erstmanifestation (DUI) eine medizinisch-psychiatrische Einrichtung innerhalb des ersten Monats nach subjektivem Erkrankungsbeginn mit voll ausgeprägter Symptomatik. Nur $8.3 \%$ der Studienteilnehmer berichteten von Kontaktaufnahme zu einer medizinisch-psychiatrischen Einrichtung vor Beginn der voll ausgeprägten Symptomatik, d.h. in der Prodromalphase. Nur 51.0\% der Studienteilnehmer kontaktierten im Rahmen eines Rezidivs (DUE) eine medizinisch-psychiatrische Einrichtung innerhalb des ersten Monats nach subjektivem Erkrankungsbeginn mit voll ausgeprägter Symptomatik. Nur 5.9\% der Studienteilnehmer berichteten von Kontaktaufnahme zu einer medizinisch-psychiatrischen Einrichtung vor Beginn der voll ausgeprägten Symptomatik, d.h. in der Prodromalphase im Rahmen eines Rezidivs. 


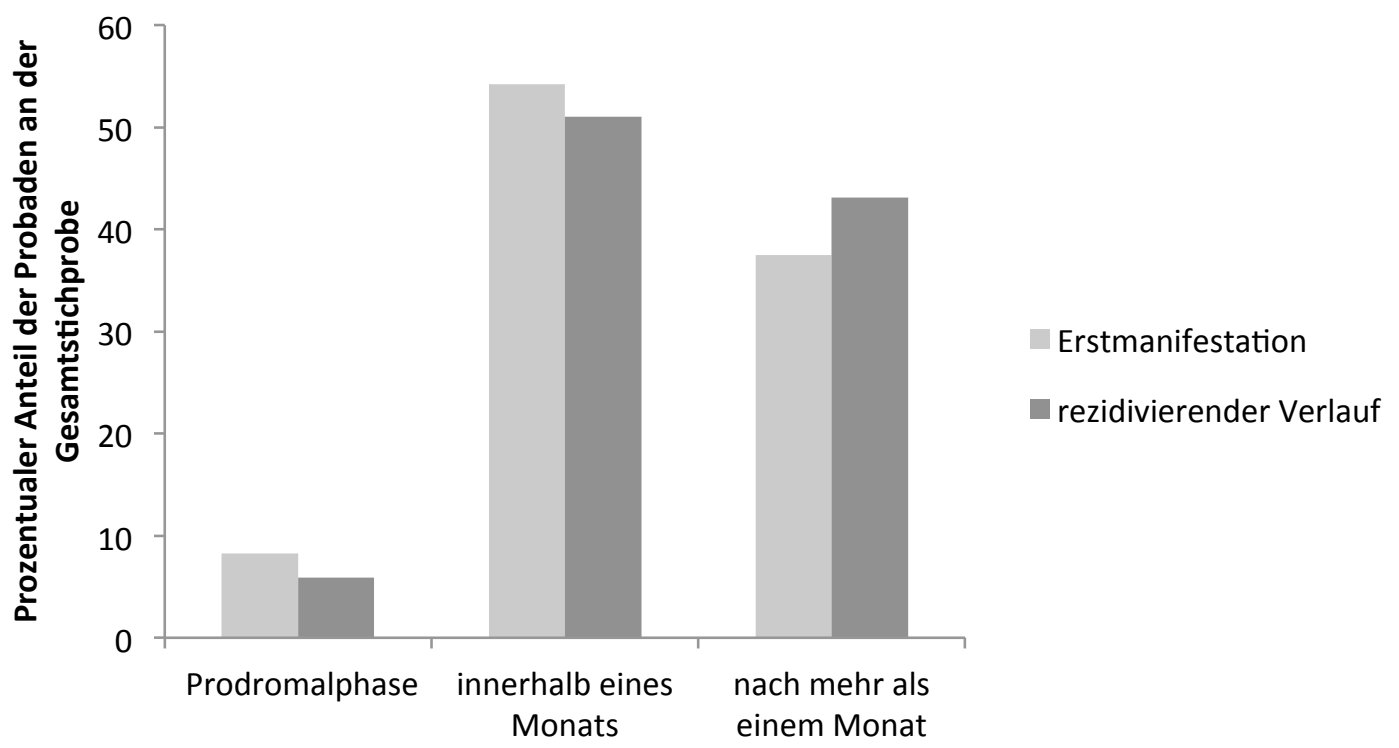

Abbildung 17. Prozentualer Anteil der Kontaktaufnahme mit einer medizinisch-psychiatrischen Einrichtung innerhalb der Prodromalphase, innerhalb eines Monats nach subjektiver Vollausprägung der Symptomatik und nach mehr als einem Monat nach subjektiver Vollausprägung der Symptomatik an der Gesamtstichprobe $(N=78)$.

Univariate Varianzanalysen zeigten keine statistisch signifikanten Unterschiede zwischen Männern und Frauen oder Altersgruppen bezüglich der DUI und der DUE und auch keine statistisch signifikanten Unterschiede in der retrospektiv berichteten Dauer der DUI oder der DUE zwischen Probanden mit akut mittelgradig bis schwerer depressiver Symptomatik und Probanden mit leichter oder gar keiner akuten depressiven Symptomatik. Es konnte keine statistisch signifikante Interaktion für die Faktoren Geschlecht und Krankheitsverlauf nachgewiesen werden. Die Dauer der DUI betrug bei Männern, welche eine Erstmanifestation aufwiesen im Durchschnitt 3.50 Monate $(S D=7.11)$. Bei Männern mit einem rezidivierendem Krankheitsverlauf betrug die durchschnittliche DUE 6.16 Monate (SD = 9.78). Für Frauen mit Erstmanifestation zeigte sich eine durchschnittliche DUI von 5.25 Monaten $(S D=10.34)$, für Frauen mit rezidivierendem Krankheitsverlauf zeigte sich eine durchschnittliche DUE von 5.56 Monaten $(S D=17.18)$. Die Mittelwerte der DUI/DUE getrennt für die Faktoren Geschlecht und Krankheitsverlauf sind in Abbildung 18 dargestellt. Es zeigte sich kein statistisch signifikanter Zusammenhang zwischen der DUI oder der DUE und der Anzahl der retrospektiv berichteten Anzahl der Episoden einer depressiven Erkrankung im Lebensverlauf. 


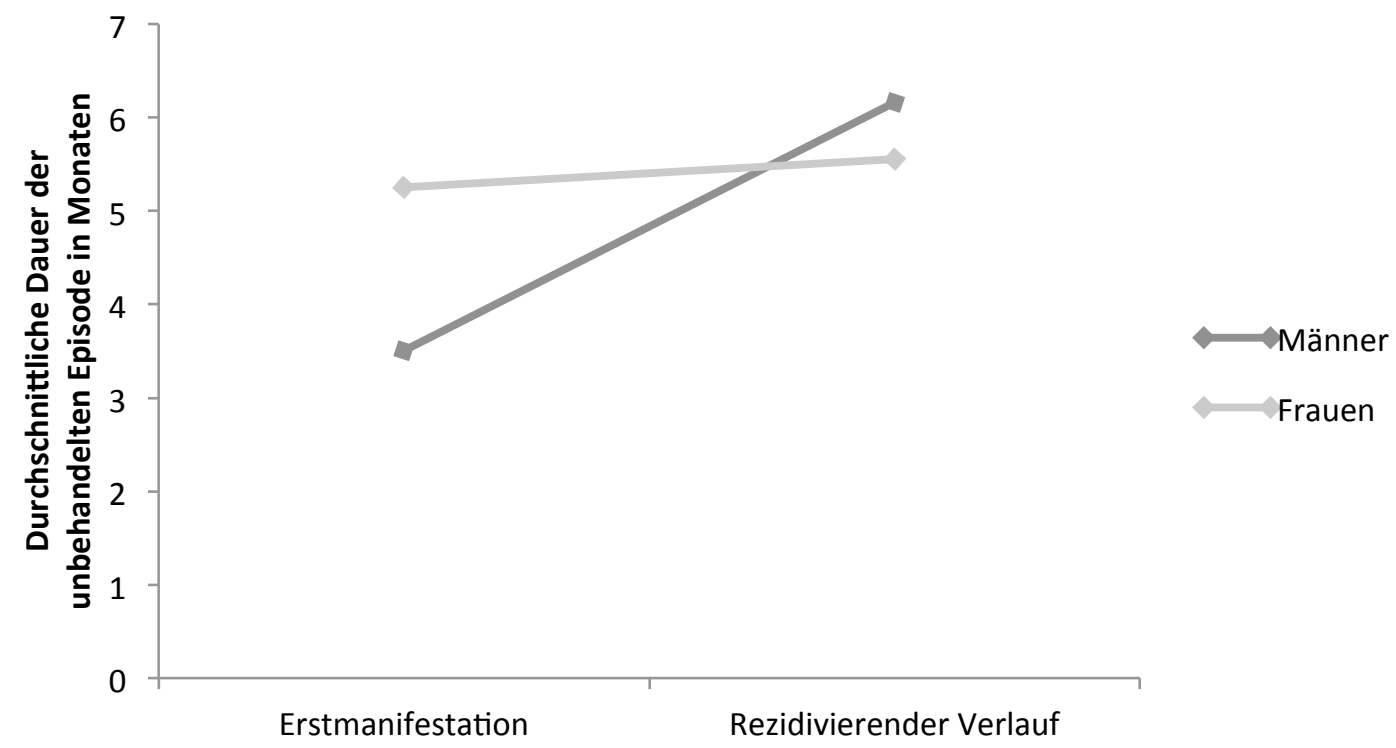

Abbildung 18. Durchschnittliche Dauer der unbehandelten Erkrankung (DUI, Erstmanifestation) und der unbehandelten Episode (DUE, rezidivierender Verlauf) getrennt für Männer und Frauen. Die Unterschiede waren statistisch nicht bedeutsam.

Persönlichkeit und Dauer der unbehandelten Episode. Die Ausprägung auf der Skala Neurotizismus korrelierte in der Gesamtstichprobe nicht signifikant mit der Dauer der DUI/DUE $(p=.33)$ in der Gesamtstichprobe. Die Ausprägung auf der Skala Extraversion korrelierte signifikant mit der DUI/DUE in der Gesamtstichprobe $(r=-.26, p=.05)$. Je höher die Ausprägung Extraversion war, desto kürzer war die DUI/DUE. Der Zusammenhang blieb auch nach Kontrolle für Geschlecht und Alter statistisch signifikant $(p<.05)$. 


\section{Diskussion}

\subsection{Interpretation und Diskussion der Ergebnisse}

In der vorliegenden Studie erfolgten eine Modellbildung zur Ätiopathogenese depressiver Erkrankungen, die Entwicklung eines daraus abgeleiteten Inventars zur Früherkennung der Depression und die Studienkonzeptualisierung zur Überprüfung und Validierung von Modell und Messinstrument. Das entwickelte Inventar wurde im Rahmen der hier dargestellten Pilotstudie eingesetzt und das Erhebungsverfahren auf Durchführbarkeit geprüft. Die Durchführbarkeit des Studienvorhabens konnte auf Grundlage der Erfahrungen aus der Pilotstudie konstruktiv diskutiert sowie Optimierungsvorschläge generiert werden. Des Weiteren konnten Erkenntnisse über spezifische Risikofaktoren depressiver Rezidive sowie über die psychopathologischen Charakteristika der Prodromalphase gewonnen werden. Die Hypothesen $\mathrm{zu}$ den angenommen Zusammenhängen zwischen spezifischen Risikofaktoren der Depression und dem Krankheitsverlauf sowie die Hypothesen zu den klinischen Charakteristika der Prodromalphase konnten in weiten Teilen bestätigt werden.

\subsection{Risikofaktoren für einen rezidivierenden Verlauf}

Psychiatrische Erkrankungen in der Familie. Die Analysen zeigten eine ausgeprägte familiäre Häufung psychischer Erkrankungen in der vorliegenden Stichprobe. Etwa 71\% der Probanden mit Erstmanifestation einer depressiven Erkrankung und etwa $76 \%$ der Probanden mit rezidivierenden Verläufen berichteten psychiatrische Erkrankungen in der Familie. Diese Raten decken sich in etwa mit den Studienergebnissen einer prospektiven Langzeituntersuchung von Weissman et al. (2016), nach welchen sich bei etwa $85 \%$ aller untersuchten Probanden mit depressiven Störungen eine depressive Erkrankung bei mindestens einem Elternteil nachweisen ließ. Die Raten speziell für depressive Erkrankungen in der Primärfamilie waren in den hier untersuchten Daten mit 38\% für Erstmanifestierte und $57 \%$ für rezidivierende Verläufe etwas geringer. Statistisch konnte kein Unterschied in der familiären Häufung psychischer Erkrankungen zwischen Probanden mit einem rezidivierenden Verlauf und einer Erstmanifestation festgestellt werden. Die geringe Stichprobenzahl und die großen Streubreiten der Daten könnten dafür verantwortlich sein, 
dass die gefundenen Unterschiede im vorhandenen Patientenkollektiv statistisch nicht bedeutsam waren. Aus den vorliegenden Daten kann demnach kein Zusammenhang zwischen einer positiven Familiengeschichte für psychiatrische Erkrankungen und dem Krankheitsverlauf geschlussfolgert werden.

Zukünftige Untersuchungen sollten ein Kontrollgruppendesign mit gesunden Probanden erwägen, da ein Vergleich der familiären Häufung psychischer Erkrankungen mit gesunden Kontrollen mehr Aufschluss über den Einfluss dieses Risikofaktors auf die Ätiologie der Depression geben kann. Ein Vergleich mit einer gesunden Kontrollgruppe war aufgrund des vorliegenden Studiendesigns an dieser Stelle nicht möglich. Auf Basis der vorliegenden Daten können daher nur eingeschränkte Schlussfolgerungen bezüglich der familiären Häufung psychischer Erkrankung gezogen werden. Eine positive Familiengeschichte kann im Rahmen dieser Studie mit Bezug zu den aus der Literatur bekannten Schätzungen nur als genereller Risikofaktor für depressive Erkrankungen angenommen werden. Eine Assoziation mit dem Krankheitsverlauf ergab sich in der vorliegenden Stichprobe nicht, was den Befunden der genannten Studien zum Einfluss der psychiatrischen Familiengeschichte auf den Krankheitsverlauf widerspricht (Lieb, Isensee, Höfler, Pfister, \& Wittchen, 2002; Weissman et al., 2016). In diesen konnte insbesondere bei Personen mit rezidivierenden Krankheitsverläufen eine familiäre Häufung psychischer Erkrankungen nachgewiesen werden. Die Familiengeschichte für psychische und im Speziellen depressive Erkrankungen sollte auch bei unbefriedigender Datenlage, die sich aus methodischen Limitationen der vorliegenden Untersuchung ergibt, auf Basis zahlreicher anderer Forschungsbefunde in ein Modell zur Früherkennung und Rezidivrisikokalkulation integriert werden. Insbesondere bezüglich der Vorhersage einer Erstmanifestation ist die Erfassung der psychiatrischen Familiengeschichte von Bedeutung, da für Kinder depressiver Eltern prospektiven Longitudinaluntersuchungen zufolge ein dreifach erhöhtes Erkrankungsrisiko im Vergleich zu Nachkommen von gesunden Probanden besteht (Weissman et al., 2016). In den vorliegenden Daten zeigte sich auch für Probanden mit einer positiven Familiengeschichte für psychiatrische Erkrankungen ein im Durchschnitt niedrigeres Ersterkrankungsalter als für Probanden in deren Familie keine psychiatrischen Erkrankungen bekannt waren. Der Unterschied war jedoch statistisch nicht signifikant. In weiteren Projektphasen zur Entwicklung des DEEP-Früherkennungsinventars sollte die psychiatrische Familienanamnese dennoch weiterhin erhoben und auf prognostische Validität geprüft werden. 
Familienstand. Der aktuelle Familienstand war in den vorliegenden Daten nicht mit dem Krankheitsverlauf assoziiert. Es ergab sich keine statistisch bedeutsame Häufung alleinstehender oder geschiedener Probanden in der Gruppe der rezidivierenden Verläufe. Dies steht in Einklang mit den diskutierten Studienergebnissen, nach welchen der Familienstand eher einen generellen Risikofaktor für die Enstehung einer Depression darstellt (Burcusa \& Icacono, 2007) und sich weniger zur Rezidivvorhersage eignet. Beachtenswert ist der margnial statistische Zusammenhang zwischen dem Familienstand und der Anzahl der retrospektiv berichteten depressiven Episoden über den Lebensverlauf. Alleinstehende oder geschiedene Probanden berichteten statistisch signifikant mehr depressive Episoden über den Lebensverlauf als Probanden in festen Partnerschaften. Feste Partnerschaften könnten in diesem Zusammenhang als protektive Faktoren für depressive Episoden interpretiert werden. Denkbar wäre aber auch, dass Probanden mit vielfachen depressiven Episoden eher ein Scheitern ihrer Partnerschaften erleben. Begründbar wäre dies mit den zahlreichen Veränderungen und Beeinträchtigungen, welche durch eine depressive Erkrankung entstehen und sich möglicherweise auch auf die Partnerschaft auswirken. Eine differenzierte Erhebung der Partnerschaftsgeschichte könnte darüber Aufschluss geben. Im DEEP-Interview wurde nur der aktuelle Familienstand erhoben, nicht die vorherige Beziehungsgeschichte.

Ersterkrankungsalter. Das mittlere Ersterkrankungsalter in der vorliegenden Stichprobe entsprach mit 27 Jahren dem berichteten Ersterkrankungsalter epidemiologischer Studien (Kessler et al., 2010). Das Ersterkrankungsalter für depressive Erkrankungen war in der vorliegenden Studie mit einem rezidivierenden Verlauf und der Anzahl der retrospektiv berichteten Episoden über den Lebensverlauf hinweg assoziiert. Probanden mit rezidivierenden Krankheitsverläufen berichteten ein deutlich niedrigeres Ersterkrankungsalter als Probanden mit nur einer berichteten depressiven Episode im Lebensverlauf. Je niedriger das Ersterkrankungsalter war, desto mehr Episoden waren zudem im Lebensverlauf nachvollziehbar. Dies entspricht den dargestellten Befunden zahlreicher Studien und spricht für einen Einfluss des Ersterkrankungsalters auf den Verlauf der Depression (z.B. Korten et al., 2012). Ein frühes Ersterkrankungsalter könnte demnach einen rezidivierenden Verlauf und eine Häufung von depressiven Episoden zu begünstigen. Für die Früherkennung der Depression bedeutet dies eine Berücksichtigung des 
Ersterkrankungsalters in der Rezidivvorhersage und -prophylaxe. Im Rahmen eines Prognoseoder Risikoalgorithmus sollte das Ersterkrankungsalter als ein gewichteter Risikofaktor integriert werden. Insgesamt sprechen die Ergebnisse der Pilotstudie dafür, die Variable Ersterkrankungsalter auch in zukünftigen Projektphasen im DEEP-Inventar zu berücksichtigen und weiter zu erforschen. Die prognostische Validität ist in weiteren Untersuchungen zu überprüfen. Der gefundene Unterschied im Ersterkrankungsalter zwischen Probanden mit depressiven Erkrankungen in der Primärfamilie und Probanden ohne positive Familiengeschichte für Depression steht in Einklang mit den Befunden aus der Literatur zum Einfluss der psychiatrischen Familiengeschichte auf die Depression (Tozzi et al., 2008). Die Ergebnisse waren in der vorliegenden Untersuchung jedoch statistisch nicht bedeutsam.

Die Ergebnisse bestätigen auch die von Kessler et al. (2009) und Uhmann und Wittchen (2010) berichteten Geburtskohorteneffekte bezüglich des Ersterkrankungsalters, nach welchen sich in den jüngeren Geburtskohorten ein früheres Ersterkrankungsalter findet. Die Ergebnisse zahlreicher retrospektiver Studien bestätigen diesen Trend (siehe Hidaka, 2013). Diskutiert werden diesbezüglich allerdings methodische Herausforderungen im Sinne verzerrter Daten bei retrospektiver Befragung (Patten et al., 2012). Leichte und lange zurückliegende Episoden werden dabei möglicherweise nicht erinnert, was eine Erklärung für den Geburtskohorteneffekt im Ersterkrankungsalter darstellen könnte. Prospektive Längsschnittstudien zeigen jedoch ähnliche Ergebnisse (Hidaka, 2013). Kritisch zu bemerken bleibt hier die Möglichkeit eines Selektionseffektes, welcher für die gefundenen Unterschiede verantwortlich sein könnte. Dass in der jüngsten Altersgruppe auch das niedrigste Ersterkrankungalter gefunden werden konnte scheint auf den ersten Blick tautologisch. Auch würde man bei frühem Erkrankungsbeginn eine höhere Wahrscheinlichkeit für Rezidive erwarten, allein aufgrund der zeitlichen Komponenten. Zu diskutieren bleibt jedoch, warum die älteren Probanden ein deutlich höheres Ersterkrankungsalter berichten. Erklärt werden könnte eine Verschiebung des Ersterkrankungsalters unter anderem zum Beispiel durch veränderte gesellschaftliche Bedingungen, circadiane Umstellungen und Schlafdeprivation, Übergewicht oder veränderte Ernährungsgewohnheiten in der heutigen Gesellschaft (Hidaka, 2013). 
Ein beachtenswertes Ergebnis ist auch der statistisch bedeutsame Unterschied im berichteten Ersterkrankungsalter für diagnostizierte und nicht-diagnostizierte Erstmanifestationen. Das Ersterkrankungsalter lag für diagnostizierte Erstepisoden deutlich höher als für subjektiv erlebte, aber nicht diagnostizierte Erstepisoden. Dies weist darauf hin, dass es in retrospektiven Erhebungen möglicherweise generell einen Unterschied zwischen dem berichteten Ersterkrankungsalter für subjektive und diagnostizierte Episoden gibt. Dies ist ebenso konsistent mit den Hypothesen zahlreicher Studien, nach welchen Menschen häufig Erinnerungs- und Abrufverzerrungen bezüglich depressiver Episoden aufweisen, insbesondere wenn es um mildere Episoden geht, die nicht ärztlich behandelt wurden (Simon \& VonKorff, 1995; Wells \& Horwood, 2004). Der Unterschied im berichteten Ersterkrankungsalter ergibt sich möglicherweise aus einer fehlenden Erinnerungsleistung für leichtere nicht diagnostizierte Episoden bei einem Teil der Befragten. Eine weitere mögliche Begründung könnte die falsche Einschätzung milderer Episoden als nicht krankheitswertig sein. Denkbar wäre auch ein Bagatellisierungseffekt, wonach einige Probanden eine Episode erst als krankheitswertig erachteten, wenn diese vom Arzt diagnostiziert wurde. Eine Konfundierung durch das Lebensalter kann hier ausgeschlossen werden, da sich die Probanden bezüglich ihres Alters bei der Datenerhebung nicht signifikant unterschieden. Schlussfolgernd lässt sich sagen, dass bei der retrospektiven Erfassung des Ersterkrankungsalters wesentliche Unterschiede zu beobachten sind, in Abhängigkeit davon, ob nach der ersten subjektiv erlebten Episode oder der ersten diagnostizierten Episode gefragt wird.

In der vorliegenden Studie wurde lediglich nach der ersten Episode im Lebensverlauf gefragt, die Differenzierung nach Diagnosestellung erfolgte mit einem nachgeschalteten Item. Dadurch konnte sichergestellt werden, dass die Probanden die erste Episode berichteten, welche sie als ausreichend schwer erinnerten, um sie als depressive Episode zu klassifizieren. Zur Früherkennung der Depression sollte ein Risikoalgorithmus auch explizit subjektiv erlebte Erkrankungsphasen extrahieren und gewichten, da unerkannte und unbehandelte Episoden wiederum das Risiko einer erneuten Erkrankung erhöhen und eine schlechtere Prognose für den Verlauf darstellen (Rost et al., 1998). Konkret bedeutet dies die Implementierung eines weiteren Items, welches im Falle einer diagnostizierten Erstepisode spezifisch nach früheren subjektiven Phasen fragt. Eine differenzierte Erfassung von subjektiv erlebten Episoden und diagnostizierten Episoden sowie behandelten und nicht 
behandelten Erstepisoden könnte in zukünftiger Forschung weiterhin Aufschluss über Erkrankungsverlauf, Prodromalphase und Dauer der unbehandelter Episoden geben. Hier ergeben sich interessante Fragestellungen, zum Beispiel bezüglich des Einflusses der Art der Erstbehandlung, des Hilfesuchverhaltens und der Dauer bis zum Erstkontakt mit einer medizinisch-psychiatrischen Einrichtung nach Ausbruch der Erkrankung. Weiterführende Analysen bezüglich des Ersterkrankungsalters sollen im Rahmen einer nachfolgenden Arbeit dargestellt werden.

Nach Interpretation der vorliegenden Daten ist das Ersterkrankungsalter von zentraler Bedeutung für die Risikokalkulation eines rezidivierenden Verlaufs. Die dargestellten Zusammenhänge sind konsistent mit bisherigen Studienergebnissen (siehe Korten et al., 2012). Je früher die Erstmanifestation auftritt, desto höher ist die Wahrscheinlichkeit eines rezidivierenden Verlaufs. Der Faktor Ersterkrankungsalter sollte daher in einen Algorithmus zur Rezidivrisikokalkulation eingehen und im weiteren Verlauf des Studienprojekts differenzierter erhoben werden. Die konkreten Vorschläge zur Optimierung des Messinstruments finden sich in Kapitel 7.6.

Neurotizismus und Extraversion. Die Ausprägung an Neurotizismus war in der vorliegenden Studie marginal signifikant mit einem rezidivierenden Verlauf der depressiven Erkrankung assoziiert. Probanden mit rezidivierenden Krankheitsverläufen wiesen im Mittel höhere Neurotizismuswerte auf als Probanden, welche bisher nur eine einzelne Episode im Lebenverlauf berichteten. Auch für die Persönlichkeitsausprägung Extraversion ließ sich nur ein margnial signifikanter Zusammenhang mit dem Krankheitsverlauf nachweisen. Die Probanden, welche in der vorliegenden Untersuchung einen rezidiverenden Verlauf der Erkrankung berichteten, wiesen geringere Extraversionswerte auf als Probaden, die eine Erstmanifestation berichteten. Extraversion korrelierte ebenso wie Neurotizsimus nicht mit der Anzahl der berichteten Episoden im Lebensverlauf. Eine Assozitation von Neurotizismus und einem rezidivierendem Krankheitsverlauf würde für die Scar-Theorie sprechen, nach der eine dauerhafte Veränderung der Persönlichkeit durch den Einfluss einer psychischen Erkrankung stattfindet. Die Veränderungen sind dem Modell zufolge auch nach der Remission persistierend und bilden einen Vulnerabilitätsfaktor für erneute Erkrankungen (Ormel et al., 2013). Die hier dargestellte Datenlage beschreibt einen Trend, der in Einklang mit den bisherigen Forschungsergebnissen für den Zusammenhang von Neurotizismus und 
dem Krankheitsverlauf depressiver Störungen steht (Kendler, Gatz, Gardner \& Pederse, 2006; Noteboom, Beekman, Vogelzangs \& Penninx, 2016). Danach ist Neurotizismus sowohl mit der Entstehung der Depression als auch dem Krankheitsverlauf assoziiert und bildet einen bedeutsamen Risikofaktor für depressive Störungen. Für die statistisch nicht bedeutsamen Ergebnisse im Rahmen dieser Studie sind möglicherweise die geringen Fallzahlen und die hohen Streubreiten der Daten verantwortlich, so dass sich hier keine definitiven Schlussfolgerungen ziehen lassen. Auf Basis der dargestellten bisherigen Forschungsbefunde sollten Persönlichkeitsmerkmale allerdings und insbesondere Neurotizismus in einem Früherkennungsinventar erfasst und in die Risikokalkulation eingebracht werden.

Die Ergebnisse zeigen weiterhin eine Assoziation von Neurotizsimus und Extraversion mit dem Ersterkrankungsalter. Wäre die Neurotizismusausprägung lediglich mit dem Ersterkrakungsalter und nicht mit dem Verlauf der Depression assoziiert, würde dies eher die Vulnerabilitäts-Hypothese unterstützen, nach welcher Neurotizismus mit Prozessen in Verbindung steht, welche den Weg für die Entwicklung einer psychischen Störung bahnen. Demzufolge werden psychische Erkrankungen entweder direkt durch eine hohe Ausprägung an Neurotizismus verursacht oder die Anfälligkeit für psychische Störungen steigt unter Einfluss von Stressoren bei gleichzeitigem Vorliegen hoher Neurotizismusausprägungen (Ormel et al., 2013). Möglicherweise sind dann gerade junge Menschen mit hohen Neurotizismusausprägungen in Transitionsphasen vulnerabel für die Entwicklung einer depressiven Störung, da Transitionsphasen im Allgemeinen eine kritische Phase im Hinblick auf die Anpassungsleistung darstellen (Ge, Conger \& Elder, 2001). Neurotizismus hat demnach möglicherweise einen moderierenden Effekt auf die Entwicklung einer depressiven Erstmanifestation im Zusammenhang mit kritischen oder stressreichen Lebensereignissen, ist jedoch nicht mit der Anzahl der folgenden Episoden oder dem Verlauf der Erkrankung assoziiert. Weitere Forschung wird nötig sein, um den differentiellen Einfluss der Persönlichkeit auf die Ätiologie der Depression und das Rezidivrisiko besser verstehen zu können. In einem Review kommen Ormel et al. (2013) zu dem Ergebnis, dass Neurotizismus eher ein Prädiktor für den Ausbruch einer psychischen Störung ist und den Zusammenhang zwischen negativen Lebensereignissen und psychischen Erkrankungen verstärkend moderiert und damit eher als unspezifischer Marker für ein generelles Erkrankungsrisiko zu werten ist. Die dargestellten Ergebnisse befürworten eher diese Hypothese, da 
Neurotizismus in der vorliegenden Untersuchung nur mit dem Ersterkrankungsalter assoziiert war, sich jedoch für den Verlauf der Erkrankung und die Episodenanzahl keine statistisch bedeutsamen Zusammenhänge nachweisen ließen.

Die gleichen Schlussfolgerungen lassen sich zudem für die Persönlichkeitsausprägung Extraversion ziehen. Die Ausprägung an Extraversion war signifikant positiv mit dem Ersterkrankungsalter assoziiert, d.h. Probanden mit hohen Werten an Extraversion wiesen ein späteres Ersterkrankungsalter auf als Probanden mit niedrigen Extraversionsausprägungen. Dies könnte für einen protektiven Mechanismus sprechen. Es gab allerdings auch hier keinen statistisch signifikanten Zusammenhang zwischen Extraversion und der Anzahl der retrospektiv berichteten Episoden oder dem Krankheitsverlauf. Der Unterschied bezüglich Extraversion war wiederum nur marginal statistisch signifikant für Probanden bei Erstmanifestation und Probanden mit einem rezidivierenden Krankheitsverlauf. Extraversion hat demnach möglichweise in der Ätiogenese der Depression eine protektive Funktion und könnte das Ersterkrankungsalter beeinflussen. Weitere Unterschungen in prospektivem Design und insbesondere unter Heranziehung einer Kontrollgruppe werden Aufschluss über den Zusammenhang zwischen der Entstehung der Depression und der Ausprägung der Persönlichkeitsvariable Extraversion liefern können.

Eine Integration der Persönlichkeitsausprägungen Neurotizismus und Extraversion in ein Früherkennungsinventar und die Relevanz im Hinblick auf einen Risikoalgorithmus scheinen trotz inkonsistenter Studienergebnisse unabdingbar. In weiteren Studien sollte ein besonderes Augenmerk auf den Zusammenhang von Persönlichkeit und der Ätiologie der Depression im Rahmen der Erstmanifestation gelegt werden. Hier ist von einem moderierenden Einfluss dieses Risikofaktors auszugehen, welcher die Wahrscheinlichkeit einer frühen Erkrankung erhöht. Ein Zusammenhang zwischen Persönlichkeit Ersterkrankungsalter und Erkrankungsverlauf wird auf Basis der vorliegenden Daten vermutet. 


\subsection{Prodromalphase}

Dauer der Prodromalphase. Die dargestellten Analysen bestätigen die Relevanz der Prodromalphase bezüglich der Früherkennung der Depression. Fast Dreiviertel aller Studienteilnehmer berichteten von psychopathologischen Veränderungen mindestens einen Monat vor Ausbruch der Erkrankung. Die Studienteilnehmer berichteten retrospektiv für die letzte Episode im Durchschnitt eine Prodromalphasendauer von neun Monaten. Dieses Ergebnis liegt innerhalb des zeitlichen Rahmens für Prodromalphasen, die in der Literatur berichtet werden (lacoviello et al., 2010). Die Angaben zu depressiven Prodromalphasen unterliegen jedoch wie bereits erwähnt je nach Studie großen Schwankungen (Jackson et al., 2003). Dies wurde auch anhand des vorliegenden Datensatzes in Form großer Streubreiten der errechneten Prodromalphasendauer deutlich. Die Datenlage spricht für eine interindividuell stark variierende Transitionphase vom Prodromalstadium in die voll ausgeprägte Erkrankung. Im Mittel findet sich in den vorliegenden Daten allerdings eine auffallend lange Prodromalphase. Wahrgenommene jedoch noch nicht als krankheitswertig interpretierte subsyndromale Symptome wurden von den Studienteilnehmern im Durchschnitt schon etwa neun Monate vor Beginn der Erkrankung wahrgenommen. Dieses Zeitfenster ließe sich im Rahmen der Früherkennung effektiv nutzen. Eine Sensibilisierung für Prodromalsymptome in der Bevölkerung scheint hier essentiell und die Länge der Prodromalphase einen präventiv nutzbaren Faktor darzustellen. Eine Schwierigkeit neben der Sensibilisierung der Betroffenen für Prodromalsymptome ist es, den Betroffenen dann auch Präventionsmaßnahmen zukommen zu lassen, um einen Ausbruch der Erkrankung zu verhindern oder den Schweregrad im Verlauf abzumildern. Personen mit depressiven Prodromalsymptomen muss ein leichterer und niederschwelliger Zugang zu medizinischer Unterstützung möglich werden. Vom ersten Frühsymptom bis zum Ausbruch der Erkrankung gibt es demnach einen großen zeitlichen Spielraum für Gegenregulation und sekundärpräventive Maßnahmen, sofern den Betroffenen erstens die Wahrnehmung der Symptome gelingt, sie zweitens entsprechendes Hilfesuchverhalten aufzeigen und die Symptome drittens von ärztlicher Seite erkannt und richtig klassifiziert werden. Eine Reduktion der Inzidenz- und Prävalenzraten kann demzufolge unter Berücksichtigung dieser drei Aspekte mit hoher Wahrscheinlichkeit gelingen.

Beachtenswert ist weiterhin der Unterschied der Prodromalphasendauer zwischen Männern und Frauen, welcher sich lediglich im Rahmen von depressiven 
Erstmanifestationen darstellt. Männer wiesen in der vorliegenden Stichprobe im Rahmen einer Erstmanifestation mit knapp zwei Jahren ein deutlich längeres Prodrom auf als Frauen mit acht Monaten. Auch war bei Männern die Prodromalphase vor einer Erstmanifestation deutlich länger als vor einem Rezidiv. Statistisch bedeutsam war dieser Effekt lediglich in der Altersgruppe der 35-49-Jährigen. In den anderen beiden Altersgruppen zeigte sich der gleiche statistische Trend. Männer berichteten bei Erstmanifestation in allen Altersgruppen längere Prodromalphasendauern als Frauen. Dieser Effekt könnte auf eine Leugnung bzw. Verkennung oder Bagatellisierung der Symptome bei Männern hindeuten. Diskutiert wird, ob die sozialen Normen der traditionellen Maskulinität möglicherweise schon die frühe Symptomwahrnehmung sowie die Reaktion auf Symptome bei Männern beeinflussen (Möller-Leimkühler, 2000). Die vorliegenden Befunde sprechen in Anbetracht der berichteten Wahrnehmung von Frühsymptomen bei Männern schon Monate bis Jahre vor der Erstmanifestation mehr für eine Bagatellisierung, Fehlinterpretation oder längere Toleranz von Missempfindungen als für eine fehlende Symptomwahrnehmung. Möglicherweise gelingt Männern im Rahmen der Erstmanifestation die Zuschreibung der Symptome zu einer psychischen Erkrankung nicht so schnell wie Frauen, was sich wiederum auf das Hilfesuchverhalten auswirken kann.

Auch der wahrgenommene subjektive Leidensdruck könnte mit der Dauer der Prodromalphase assoziiert sein. So könnten die Prodromalsymptome für Männer und Frauen möglicherweise einen unterschiedlichen Grad an Leidensdruck erzeugen. Auch das Eingestehen einer Depression mag unter traditionellen geschlechtsspezifischen Normen und der gesellschaftlichen Beschreibung einer Depression, welche vornehmlich weibliche Attribute beinhaltet (Möller-Leimkühler, 2008), Männern schwerer fallen als Frauen. Die veränderte Ergebnislage für die Gruppe der rezidivierenden Verläufe würde für die erste Hypothese sprechen. Im Rahmen eines Rezidivs findet sich kein Unterschied mehr zwischen Männern und Frauen bezüglich der Länge der Prodromalphase. Erleben Männer eine ähnliche Symptomatik erneut, kann eine Zuschreibung zu einer depressiven Erkrankung möglicherweise schneller gelingen als im Rahmen einer Erstmanifestation. Die Barriere für Hilfesuchverhalten mit vermeintlich weiblichen Symptomen ist dahingehend geringer, da sie bereits früher schon überschritten wurde. Es findet eine Veränderung im Hilfesuchverhalten statt. 
Für Probanden mit akut mittelgradig bis schwerer depressiver Symptomatik und Probanden ohne akute oder mit leicht depressiver Symptomatik ergaben sich hier keine Unterschiede in der berichteten Dauer der Prodromalphase. Dies spricht gegen eine Verzerrung der Daten aufgrund akut depressiver Symptome, zumindest für den Faktor der retrospektiv berichteten Prodromalphasendauer. Die Dauer der Prodromalphase bildet zwar auf Basis der zugrundeliegenden Daten keinen geeigneten Prädiktor für die Anzahl der Episoden im Lebensverlauf oder für den Krankheitsverlauf ist jedoch wesentlich für die Abschätzung eines sekundärpräventiv nutzbaren Zeitrahmens bis zum Ausbruch einer möglichen Erkrankung. In weiteren Untersuchungen könnten Prodromalphasendauer und Prodromalsymptome noch genauer analysiert werden. Interessant wäre zum Beispiel der Symptomverlauf im depressiven Prodrom. Daraus ließe sich möglicherweise ein sehr individuelles und zeitlich exaktes Zeitfenster für sekundärpräventive Maßnahmen bestimmen. Auch könnte es im Rahmen der Prodromalphase besonders sensible Phasen für erfolgreiche Interventionen geben, zum Beispiel wenn die Symptomschwere noch mäßig ausgeprägt ist, aber der Leidensdruck schon ein ausreichendes Maß erreicht hat und damit eine Veränderungsmotivation besteht. Dies könnte im Rahmen der Prävention der Depression sowohl für Erstmanifestationen als auch Rezidive nützlich sein. Zu vermuten ist, dass sich unterschiedliche Prodromaltypen ergeben, die sich in Länge sowie Symptomkonstellation und -schwere unterscheiden. Daraus ließen sich dann im Anschluss darauf abgestimmte Präventivmaßnahmen ableiten. Die nachfolgenden Untersuchungen im Rahmen dieses Projekts sollten die Erfasssung und Analyse der Prodromalphase in detailierterer Form berücksichtigen.

Prodromalsymptome. Die Ergebnisse der qualitativen Auswertung der berichteten Symptome in der Prodromalphase decken sich mit den Ergebnissen bisheriger Studien (Fava, Grandi, Zielezny, Canestrari \& Morphy, 1994; Fava \& Kellner, 1991; Syed Sheriff et al., 2015). Als Prodromalsymptome wurden sowohl Symptome in Übereinstimmung mit den diagnostischen Kriterien der Depression gefunden, als auch Symptome außerhalb dieses Spektrums. Am häufigsten fanden sich somatische Beschwerden oder körperliche Missempfindungen, Schlafstörungen sowie Ängste und Sorgen, gedrückte Stimmung und Erschöpfung. Fast die Hälfte aller Probanden gaben Schlafschwierigkeiten im Rahmen der Prodromalphase an. Mehr als die Hälfte aller Studienteilnehmer berichtete somatische Beschwerden. Darunter am häufigsten sehr individuelle körperliche Missempfindungen oder 
vegetative Veränderungen wie zum Beispiel vermehrter Juckreiz, Gefühl eines Kloßes im Hals, Gefühl einer Erkältung oder Kribbeln im Körper. Auffallend war hier auch die Anzahl an berichteten Schmerzempfindungen. Knapp ein Fünftel aller Probanden mit somatischen Beschwerden berichtete von Schmerzen innerhalb der Prodromalphase. Ebenso wurden Ängste und Sorgen, gedrückte Stimmung sowie Erschöpfung und Müdigkeit von fast einem Viertel aller Befragten angegeben. Vermehrt wurden zudem Symptome wie Antriebs- und Energieminderung, Grübeln, emotionale Labilität und Empfindsamkeit, sozialer Rückzug, Konzentrationsstörungen, Reizbarkeit, Überforderungserleben sowie Nervosität und Unruhe berichtet. Weiterhin berichteten die Probanden Veränderungen des Appetits sowie MagenDarm Beschwerden. Sehr vereinzelt wurden Aggressivität und Wut, verminderte Selbstwertgefühle, vermehrter Substanzgebrauch, Anspannung, Schuldgefühle, zwanghafte oder wahnhafte Symptome, dysfunktionale Gedanken und Libidoverlust berichtet. Die Ergebnisse weisen auf eine ausgeprägte Somatisierung im Rahmen des depressiven Prodroms hin. Dies unterscheidet sich von den bisher in der Literatur beschriebenen Prodromalsymptomen (Fava et al., 1990). Der Unterschied ergibt sich vermutlich aus der Konzeptualisierung des verwendeten Erhebungsinstruments. Das DEEP-Interview kategorisiert die Prodromalsymptome in psychische und körperliche Frühsymptome, welche nacheinander abgefragt werden. Es ist davon auszugehen, dass die Betroffenen somatische Symptome in der retrospektiven Erhebung im freien Abruf, also ohne Vorgabe der Kategorie körperliche Frühsymptome möglicherweise nicht der Depression zugeordnet oder nicht erinnert und damit nicht berichtet hätten. Die Vorgabe und gezielte Nachfrage nach körperlichen Frühsymptomen im DEEP-Interview bildeten damit einen guten Abrufanker.

Ein beachtenswertes Ergebnis ist weiterhin der Geschlechterunterschied in der Auftretenswahrscheinlichkeit körperlicher Missempfindungen in der Prodromalphase. Die Wahrscheinlichkeit lag in der vorliegenden Stichprobe für Frauen deutlich höher in der Prodromalphase körperliche Missempfindungen zu erleben als für Männer. Dabei handelte es sich in den meisten Fällen, wie oben beschrieben, um sehr individuelle und spezielle körperliche Symptome. Auch berichteten Frauen im Rahmen der Prodromalphase deutlich häufiger von Symptomen emotionaler Labiliät und Empfindsamkeit. In allen anderen Symptomkategorien fanden sich keine Unterschiede in den berichteten Häufigkeiten zwischen Männern und Frauen. Dies spricht für eine zumindest teilweise genderspezifische Prodromalsymptomatik. 
Weiterhin beachtenswert und konsistent mit Studienergebnissen zu depressiven Symptomen im höheren Lebensalter (Korten et al., 2012) ist die gefundene Häufigkeit von berichteten Appetitveränderungen bei älteren Menschen im Rahmen der Prodromalphase. Die Chance innerhalb der Prodromalphase Appetitveränderungen zu erleben war für die Altersgruppe der 50-64-Jährigen um das Fünfache erhöht im Vergleich zu den jüngeren Altersgruppen. Eine Erklärung dafür könnten allerdings die mit dem Altern einhergehenden generellen Veränderungen des Appetits sein (Donini, Savina \& Cannella, 1999). Die nachfolgenden Untersuchungen im prospektiven Design werden dazu weiteren Aufschluss geben. Insgesamt scheinen die Prodromalsymptome in weiten Bereichen für Männer und Frauen und unterschiedliche Altersgruppen gleich zu sein, es finden sich nur in einzelnen Symptomclustern Unterschiede in den berichteten Häufigkeiten. Die Notwendigkeit einer gender- und altersgruppenspezifischen Analyse und letztlich auch Klassifikation der Prodromalphase wird hier dennoch deutlich, da gerade die Unterschiede in den einzelen Kategorien einen maßgeblichen Einfluss auf den Erkrankungsverlauf haben könnten.

Ein zentraler und zu diskutierender Aspekt bezüglich der retrospektiven Erinnerung von Prodromalsymptomen ist auch die in neuesten Studien nachgewiesene Beeinträchtigung kognitiver Funktionen, insbesondere die Veränderungen des autobiographischen, episodischen Gedächtnisses im Rahmen depressiver Erkrankungen (Soderlund et al., 2014). Ein eingeschränktes Erinnerungsvermögen an frühere Symptome und Episoden sowie mangelnde metakognitive Fähigkeiten könnten die eigene Wahrnehmung vor allem von Frühwarnzeichen beeinträchtigen (Hargus, Crane, Barnhofer \& Williams, 2010). Damit steht für Probanden mit akut depressiver Symptomatik die Qualität der erhobenen Daten in Frage. Auf Basis der vorliegenden Daten zeigten sich jedoch nur geringfügige Unterschiede in den berichteten Prodromalsymptomen zwischen Probanden ohne oder mit akut leichter depressiver Symptomatik und Probanden mit akut mittelgradig bis schwerer depressiver Symptomatik. Probanden mit akut mittelgradiger und schwerer Symptoamtik berichteten retrospektiv marginal mehr körperliche Missempfindungen und marginal weniger Appetitveränderungen für die Prodromalphase der letzten depressiven Episode. In allen anderen Kategorien ergaben sich hier keine Unterschiede. Zur Sicherstellung einer valideren Datenlage sollte sich in zukünftigen Studien die Datenerhebung auf zumindest teilremittierte depressive Patienten beschränken. Allerdings wären in zukünftigen Studien auch weitere Vergleiche der retrospektiv berichteten klinischen Charakteristika der Prodromalphase 
zwischen akut depressiven und remittierten Patienten interessant, da sich hier auch therapeutische Implikationen für die Rezidivprophylaxe ergeben könnten.

Dauer der unbehandelten Episode (DUE). Die Reduktion der durch Depressionen verursachten gesellschaftlichen Kosten kann zum einen über die Reduktion der Erkrankungsraten im Sinne der Primärprävention geschehen, zum anderen aber auch über eine Sekundärprävention im Sinne einer zeitnahen Behandlung von Betroffenen. Die Dauer der unbehandelten Erkrankung oder Episode lag in der vorliegenden Stichprobe im Durchschnitt bei fünf bis sechs Monaten sowohl für Erstmanifestationen als auch für Rezidive. Nur 54.2\% der Studienteilnehmer kontaktierten im Rahmen einer Erstmanifestation eine medizinisch-psychiatrische Einrichtung innerhalb des ersten Monats nach subjektivem Erkrankungsbeginn mit voll ausgeprägter Symptomatik und nur knapp acht Prozent der Studienteilnehmer berichteten von Kontaktaufnahme zu einer medizinischpsychiatrischen Einrichtung vor Beginn der voll ausgeprägten Symptomatik, d.h. in der Prodromalphase. Etwa die Hälfte der Studienteilnehmer kontaktierte im Rahmen eines Rezidivs eine medizinisch-psychiatrische Einrichtung innerhalb des ersten Monats nach subjektivem Erkrankungsbeginn mit voll ausgeprägter Symptomatik und nur etwa sechs Prozent der Studienteilnehmer berichteten von Kontaktaufnahme zu einer medizinischpsychiatrischen Einrichtung vor Beginn der voll ausgeprägten Symptomatik, d.h. in der Prodromalphase eines Rezidivs. Diese Zahlen sprechen für eine Hemmschwelle bei den Betroffenen, schon in der Prodromalphase und sogar nach Ausbruch der Erkrankung medizinische Hilfe in Anspruch zu nehmen. Dies lässt sich möglicherweise durch die nach wie vor weit verbreitete Stigmatisierung psychiatrischer Symptome und Erkrankungen in der Gesellschaft begründen. Möglicherweise steht einer frühzeitigen Kontaktaufnahme mit fachärztlichen Einrichtungen die Angst vor Stigmatisierung oder auch ein Gefühl von Scham entgegen (Angermeyer, 2003; Reynders, Kerkhof, Molenberghs \& van Audenhove, 2014). Auch eine Bagatellisierung der Symptome oder eine unzureichende Aufklärung der Bevölkerung über frühe Anzeichen und Symptome der Depression könnten für die geringe Inanspruchnahme medizinischer Unterstützung in der Prodromalphase und der ersten Zeit nach Ausbruch der Erkrankung verantwortlich sein. Eine somatische Präsentation depressiver Frühsymptome könnte ein weiterer Grund für das verzögerte Hilfesuchverhalten sein, da keine Zuordnung der Symptome zu einer psychischen Belastung stattfindet und eine psychische Erkrankung verkannt wird. Ziel präventiver Arbeit sollte es demnach sein, die 
Betroffenen für die Prodromalsymptome der Depression zu sensibilisieren und ihnen schon in der Phase vor dem Beginn der voll ausgeprägten Symptome eine Unterstützung anzubieten, um den Schweregrad und die Dauer der Erkrankung abzumildern, oder den Ausbruch der Erkrankung gar gänzlich zu verhindern. Bei Erkrankungsbeginn sollte die Kontaktaufnahme mit medizinisch-psychiatrischen Einrichtungen früher ermöglicht werden, da sich die Dauer der unbehandelten Erkrankung nachweislich auch auf den Verlauf der Depression auswirken kann (Ghio et al., 2015).

Die Ergebnisse sprechen weiterhin für eine Umstrukturierung der präventionsstrategischen Überlegungen bezüglich depressiver Erkrankungen. Die Implikation der vorliegenden Datenlage für die klinische Praxis richtet sich an zwei zentrale Probleme der Gesundheitspolitik: Zum einen ergibt sich eine Forderung nach dem Bemühen der Entstigmatisierung psychischer Erkrankungen in der Gesellschaft und zum anderen nach einer besseren Aufklärung nicht nur über die Symptomatik der Depression sondern auch über mögliche Frühwarnzeichen und Risikofaktoren. Dies spricht für die Etablierung und den Ausbau entsprechender Präventionszentren sowie einer Regelung abrechnungstechnischer Aspekte bei Nichtvorliegen einer vollausgeprägten psychischen Erkrankung und dem Angebot an interventionellen Maßnahmen. Hier stellt sich die Frage nach der Kostenübernahme von primär- und sekundärpräventiven Maßnahmen. Dies ist ein Aspekt der in Zukunft intensiver gesundheitspolitisch diskutiert werden muss.

\subsection{Durchführbarkeit der Studie}

Insgesamt lassen sich Durchführ- und Machbarkeit des vorliegenden Studienvorhabens bestätigen. Sowohl aus inhaltlicher Sicht als auch unter Berücksichtigung der Rahmenbedingungen kann von einer guten Durchführbarkeit der dargestellten Konzeption ausgegangen werden. Mit allen Probanden konnte das DEEP-Interview vollständig durchgeführt werden, ein Großteil der Probanden sandte die ausgehändigten Fragbögen vollständig ausgefüllt zurück. Der zeitliche Aufwand, welcher mit der Studienteilnahme verbunden war, wurde von den Probanden akzeptiert. Es gab dahingehend keine Auffälligkeiten. Auch konnte keine Überlastung mit Auswirkungen auf die Konzentration oder Aufmerksamkeit der Probanden im Rahmen des Interviews festgestellt werden. Für die Bearbeitung der Fragbögen lassen sich diesbezüglich jedoch keine Aussagen machen, da hier 
aufgrund des Bearbeitungssettings keine Evaluation stattfinden konnte. Für weitere Studienphasen, insbesondere für die retrospektive Querschnitterhebung zur Skalenkondensation (Phase 1c) könnte die subjektive Belastung und der zeitliche Aufwand der Fragebogenbearbeitung zur Qualitätssicherung erfasst werden, zumal die Risikokalkulation der Depression wie dargestellt ein komplexer und vielschichtiger Prozess ist, in dem unterschiedlichste Parameter berücksichtigt werden müssen. Die Erfassung der zeitlichen Rahmenbedingungen und der subjektiven Belastung durch die Fragebogenbearbeitung könnten dabei helfen, einen bezüglich der Belastbarkeit festgelegten Maximalumfang des DEEP-Inventars zu bestimmen. Einen Kompromiss zwischen der weitgefächerten Erfassung möglichst vieler Risikofaktoren und der ökonomischen und zumutbaren Umsetzung zu finden, bleibt eine große Herausforderung in der Forschung zur Früherkennung der Depression. Ziel ist es im Rahmen dieses Projekts die essentiellen Risikoparameter herauszufiltern, um so ein effizientes und zugleich ökonomisches Verfahren zur Früherkennung der Depression gewährleisten zu können.

Bezüglich der Zumutbarkeit des Datenerhebungsverfahrens ist zu ergänzen, dass kein Studienteilnehmer das Interview frühzeitig beendete oder unterbrach. Daraus lässt sich eine Bereitschaft der Patienten, an einem zweistündigen Interview teilzunehmen schlussfolgern und auf eine Zumutbarkeit des dargestellten Erhebungsverfahrens schließen. Alle Teilnehmer wurden vor Beginn der Untersuchung über die Möglichkeit zur Unterbrechung oder des Abbruchs der Untersuchung aufgeklärt und bestätigten schriftlich über diese Möglichkeit ausreichend aufgeklärt worden zu sein. Auf eine Evaluation der Zumutbarkeit in Form standardisierter Messinstrumente wurde in der vorliegenden Studie verzichtet. Dieses Vorgehen hätte einen fundierten Schluss über die Zumutbarkeit des Datenerhebungsprozesses gewährleistet. Gegen dieses Vorgehen sprachen der bereits starke Umfang des DEEP-Inventars und die damit verbundene hohe Durchführungsdauer. Eine Evaluation des Erhebungsprozesses hätte eine weitere zeitliche und kognitive Inanspruchnahme der Probanden bedingt. Zugunsten dieser Faktoren wurde im Rahmen der vorliegenden Studie auf die Evaluation des Prozesses verzichtet, zumal das übergeordnete Ziel des Projekts in einer Skalenkondensation und Itemreduzierung liegt und das zu konstruierende Instrument in seiner Endversion wesentlich kürzer gestaltet werden soll. Bezüglich der Fragebögen, welche die Teilnehmer zuhause ausfüllten, lässt sich die Zumutbarkeit ebenfalls diskutieren. Die Bearbeitung der Fragebögen nahm den Messungen 
im Rahmen einer Voruntersuchung zufolge in etwa zwei Stunden in Anspruch. Die Rücksendequote betrug 75\%, was als akzeptabel zu bewerten ist. Die finanzielle Aufwandsentschädigung nach Auszahlung der ausgefüllten und rückgesendeten Fragebögen trug hier mit hoher Wahrscheinlichkeit wesentlich zur dargestellten Rücksendequote bei. Auf eine Evaluation der Fragebogenbearbeitung im Sinne der Zumutbarkeit wurde auch hier aus den oben genannten Gründen verzichtet.

Im Rahmen der Durchführbarkeit ist die auffallend geringe Teilnehmerquote der hier dargestellten Pilotstudie zu diskutieren. Von insgesamt 1363 zur Studienteilnahme eingeladenen Patienten erklärten sich lediglich 85 Personen zur Teilnahme bereit. Die auffallend niedrige Quote lässt sich vielschichtig erklären. Zum einen ergab sich im Rahmen des schriftlichen Rekrutierungsverfahrens eine hohe Anzahl an nicht zustellbaren Briefen (ca. 20\%). Die Postanschriften der ehemaligen Patienten könnten in vielen Fällen inkorrekt gewesen sein. Eine fehlerhafte Adressenerfassung im Kliniksystem, Falsch-Angaben der Patienten und/oder eine hohe Fluktuation von Wohnsitzen in psychiatrischen Patientenkollektiven könnten mögliche Erklärungen dafür sein. Weiterhin lässt sich die geringe Teilnahmequote mit einer mangelnden Bereitschaft der ehemaligen Patienten zur erneuten Auseinandersetzung und gedanklichen Reflexion mit der depressiven Erkrankung begründen. Die Angst vor einem erneuten Erleben der Symptome durch gedankliche Beschäftigung mit der Erkrankung kann vermutet werden. Möglicherweise spielen hier aber auch Befürchtungen der Stigmatisierung eine zentrale Rolle, insbesondere da es sich um einen psychiatrischen Kontext handelt und die Datenerhebung in einer psychiatrischen Klinik stattfand. Eine Erhebung in einem nicht-psychiatrischen Kontext wäre bezüglich der Teilnahmebereitschaft denkbar günstiger. Aus der Durchführung der Pilotstudie lässt sich zudem ableiten, dass die Teilnahmebereitschaft bei persönlichem Ansprechen der Patienten im Rahmen ihres stationären Aufenthalts höher war als bei Personen, welche aktuell nicht mehr an die Klinik angebunden waren. Im Rahmen einer persönlichen Einladung zur Studienteilnahme lehnte nur ein Viertel der Patienten die Teilnahme ab, im Vergleich zu Dreiviertel der Probanden bei schriftlicher Einladung. Zukünftige Datenerhebungsverfahren im Kliniksetting sollten daher die persönliche Rekrutierung von Probanden forcieren.

Die Aufnahme eines Fahrtwegs könnte möglicherweise ein weiterer Grund für die geringere Teilnahmebereitschaft auf die schriftliche Einladung sein. Die 
Aufwandsentschädigung in Höhe von 40 Euro kann als Faktor zur Beeinflussung der Teilnahmebereitschaft ausgeschlossen werden, da weder die mündliche noch die schriftliche Einladung den Hinweis einer Aufwandsentschädigung enthielt. Dieses Vorgehen wurde von den Studienleitern gewählt, um Verzerrungen durch finanzielle Anreize in der Zusammensetzung der Stichprobe zu reduzieren. Eine im Vorfeld angekündigte Aufwandsentschädigung könnte im zukünftigen Datenerhebungsprozess des Projekts die Teilnahmequote jedoch möglicherweise verbessern. Abzuwägen bleibt hier die genannte Verzerrung der Stichprobenzusammensetzung bei Ankündigung einer finanziellen Aufwandsentschädigung. In Anbetracht der geringen Teilnahmequote sollte im weiteren Verfahren eventuell zu Gunsten der Erzielung höherer Fallzahlen entschieden werden und eine Aufwandsentschädigung vor Studienteilnahme transparent gemacht werden.

\subsection{Stärken und Limitationen}

Stärken. Stärken der Studie sind insbesondere die ausführliche und umfassende Erhebung anamnestischer und soziodemographischer Daten anhand des hier konzipierten DEEP-Interviews. Das DEEP-Interview erlaubt eine retrospektive Erfassung des gesamten Krankheitsverlaufs über die Lebenszeit, eine Erfassung des Ersterkrankungsalters, aller Haupt- und Frühsymptome der zeitlich letzten Episode, die Erfassung der Dauer der Prodromalphase der letzten Erkrankungsphase und der Dauer der unbehandelten Erkrankung vor Kontaktaufnahme mit einer medizinisch-psychiatrischen Einrichtung. In der vorliegenden Studie wurde damit ein geeignetes Messinstrument zur Überprüfung der zugrunde liegenden Fragestellungen verwendet. Das DEEP-Interview erlaubt zudem eine Klassifikation des Krankheitsverlaufs der zugrunde liegenden Störung. Durch eine zusätzliche Erhebung soziodemographischer Variablen und eine integrierte psychiatrische Familienanamnese lassen sich epidemiologische Fragestellungen anhand des konzipierten Messinstruments überprüfen und mögliche Risikofaktorinteraktionen in der Ätiopathogenese der Depression berücksichtigen.

Eine weitere Stärke der vorliegenden Studie ist die umfassende und weit gefächerte Erhebung möglicher Risikofaktoren depressiver Erkrankungen. Die Integration multipler Risikofaktoren sowie deren Interaktionen in ein Ätiologiemodell können nur gelingen, wenn 
Heterogenität und Komplexität der Depression in theoretischen und methodischen Modellen berücksichtigt werden. Dies ist eine große Herausauforderung, welcher sich das hier dargestellte Studienprojekt anzunähern versucht. Soweit bekannt, wurde ein derart umfangreiches Früherkennungsmodell empirisch bislang nicht geprüft. Die hier dargestellten theoretischen und methodischen Überlegungen und die Analyse der ersten Ergebnisse der Pilotstudie bilden den Grundstein für eine weitere fundierte Früherkennungsforschung.

Limitationen. Bezüglich der Stichprobe lassen sich die niedrige Fallzahl sowie die Zusammensetzung der Stichprobe diskutieren. Alle Probanden waren ehemalige oder aktuelle Patienten in psychiatrischen oder psychosomatischen Kliniken. Der Hauptanteil der Stichprobe wurde aus einer psychiatrischen Klinik rekrutiert. Die Zusammensetzung der Stichprobe erlaubt daher keine Rückschlüsse auf ambulante oder hausärztlich behandelte Personen mit depressiven Erkrankungen. Bezüglich der Stichprobe ist somit ein möglicher Selektionseffekt anzumerken, welcher sich aus der Schwerpunktrekrutierung im psychiatrischen Setting begründen lässt. Die Repräsentativität der Daten steht damit in Frage.

Die niedrige Fallzahl begründet sich aus der auffallend geringen Rückmeldung auf die Einladungen zur Studienteilnahme sowie auf den beträchtlichen Umfang des Datenerhebungsverfahrens. Die vollständige Erfassung, Aufbereitung und Verarbeitung eines Datensatzes bedarf hoher zeitlicher und personeller Ressourcen. Eine Verkürzung der zeitlichen Abläufe könnte die Fallzahlen positiv beeinflussen, würde jedoch die Qualität und den Umfang der erhobenen Informationen beeinträchtigen. Die niedrige Stichprobenzahl erlaubte weiterhin keine detaillierten Vergleiche zwischen einzelnen Störungsbildern und Diagnosegruppen, da die einzelnen Zellbesetzungen zu niedrig waren. In zukünftigen Studien sollte eine differenzierte Analyse der dargestellten Risikofaktoren und ProdromalphasenCharakteristika getrennt nach Diagnosegruppen erfolgen.

Anzumerken sind weiterhin mögliche Optimierungen des Studiendesigns. Das Design umfasst keine Kontrollgruppe, die Gruppenvergleiche wurden anhand der ex-post-facto gebildeten Gruppen erstellt. Ein Vergleich der untersuchten Variablen mit einer gesunden Stichprobe war daher nicht möglich, wäre jedoch für zukünftige Forschung erstrebenswert und notwendig zur Überprüfung des hier konstruierten Modells zur Ätiologie der Depression. 
Bezüglich der Rekrutierung ist die Diagnosestellung bei vorliegendem Patientenkollektiv weiterhin kritisch anzumerken. Die Diagnosen wurden lediglich aus den von den Patienten vorgelegten Arztbriefen entnommen. Es erfolgte keine eigene klinische Diagnosestellung. Für einen Verzicht der Diagnosebestätigung im Rahmen der vorliegenden Untersuchung sprach der Aspekt, die Verweigererquote bei einem ohnehin sehr umfangreichen Erhebungsverfahren niedrig zu halten. Ein zusätzliches diagnostisches Interview hätte eine zu starke zeitliche Belastung der Probanden mit sich gebracht und die Compliance sowie die Reliabilität der Daten aufgrund von motivationalen und konzentrationsbedingen Faktoren in Frage gestellt.

Das vorliegende Studiendesign erlaubt weiterhin keine kausalen Schlussfolgerungen, es handelt sich um eine Querschnitterhebung und rein korrelative Daten. Kausale Zusammenhänge über Risiko- und Schutzfaktoren sowie Prodromalsymptome der Depression werden in zukünftigen Untersuchungen mittels prospektiver Längsschnittstudien zu überprüfen sein. Erwähnenswert ist weiterhin die rein retrospektive Datenerhebung. Eine Verzerrung der Erinnerungsleistung bei depressiven Patienten wird in der Literatur seit Langem diskutiert (Brewin, Andrews \& Gotlib, 1993). Die empirischen Befunde zeigen unter Anderem eine Aggravierung der Symptomschwere, eine verstärkte Abruftendenz für negative Stimuli, einen eher generalisierten Gedächtnisabruf sowie verzerrte Attributionsund Kognitionsstile bei retrospektiven Berichten depressiver Patienten (Ben-Zeev, Young \& Madsen, 2009). Henry, Moffitt, Caspi, Langley et al. (1994) gehen davon aus, dass die retrospektive Datenerhebung im Allgemeinen fehleranfällig ist, da viele Ereignisse vergessen werden, insbesondere wenn sie weit in der Vergangenheit liegen. Ereignisse werden demnach häufig zeitlich näher eingeordnet, als sie tatsächlich waren und die Vergangenheit häufig den aktuellen Zuständen und Bedürfnissen entsprechen umstrukturiert (Henry et al., 1994). Damit wäre die retrospektive Datenerhebung an sich fehleranfällig und bei Vorliegen einer depressiven Erkrankung noch zusätzlich verzerrt. Die Interpretation retrospektiver Daten und die Ableitung theoretischer und klinischer Schlussfolgerungen ist trotz der genannten Einwände eine in Forschung und Praxis etablierte und häufig genutzte Methode (Henry et al., 1994). Die Überprüfung der dargestellten Hypothesen und Befunde sollte im weiteren Studienverlauf und zukünftiger Forschung anhand prospektiver Studien erfolgen. 


\subsection{Optimierung des DEEP-Inventars}

Für das DEEP-Interview ergeben sich aus der Durchführung der Pilotstudie Verbesserungsvorschläge. Diese sollen im Folgenden dargestellt werden.

Prodromalsymptome. In der weiteren Studienkonzeptualisierung sollte eine Optimierung der Erhebung der Prodromalsymptome angestrebt werden. Die Symptome der Prodromalphase wurden in der dargestellten Untersuchung im offenen Format, kategorisiert nach psychischen und körperlichen Frühsymptomen erfragt und von den Probanden im freien Abruf berichtet. Möglicherweise unterliegen die Ergebnisse hier Abruffehlern, im Sinne eines Nichterinnerns an alle erlebten Symptome. Eine verbesserte Vorgehensweise wäre dabei die Ergänzung der Erhebung um eine standardisierte Prodromalsymptomcheckliste, welche auf Basis der hier dargestellten Ergebnisse und den in der Literatur bisher diskutierten Frühsymptomen zusammengestellt werden könnte. In Anlehnung an die Vorgehensweise der qualitativen Studie von Syed Sheriff et al. (2015) wäre die Abfrage von Frühsymptomen zur Wiedererkennung dem offenen Antwortformat nachgeschaltet. Damit würden Erinnerungsfehler minimiert und eine Kategorisierung der Symptome vereinfacht werden. Eine Kombination aus freiem Abruf und einer nachgeschalteten Wiedererkennug wäre hier eine zu erwägende Veränderung des DEEPInterviews und könnte zu valideren Ergebnissen führen. Die Erfassung des Schweregrads der genannten Symptome erfolgt im Anschluss daran anhand des DEEP-A Fragebogens zur Prodromalsymptomatik.

Anzahl der Prodromalsymptome. Ein weiterer Optimierungsvorschlag bezieht sich auf die Vorgabe der Anzahl der erhobenen Frühsymptome. Die in der aktuellen Version des DEEP-Interviews vorgegebene Anzahl der Frühsymptome wirkt sich limitierend auf die Qualität der Daten aus. Im Rahmen des DEEP-Interviews wurden jeweils drei körperliche und drei psychische Frühsymptome erfragt. Eine Unterscheidung der Frühsymptome kann daher nur auf qualitativer Ebene stattfinden. Die Anzahl der erlebten Frühsymptome wurde nicht erfasst, da sich die Vorgabe auf jeweils drei zu nennende Symptome beschränkte. Anzunehmen sind jedoch auch Unterschiede in der Anzahl der erlebten Frühsymptome und ein Zusammenhang dessen mit der Dauer der Prodromalphase. Dahingehend sollte das Interview ergänzt und die Items zur Erfassung der Frühsymptome umgestaltet werden. Zu empfehlen wäre die Fortführung einer Vorgabe der Kategorien körperliche und psychische 
Frühsymptome, allerdings eine Aufhebung der Limitierung der Anzahl der zu berichtenden Symptome. Ein freier Abruf sollte hier instruiert werden. Um Suggestionseffekten vorzubeugen sollte die Instruktion des Items zweifachgestuft sein, z.B.:

1. „Bemerkten Sie körperliche Frühsymptome?"

2. „Wenn ja, welche körperlichen Frühsymptome bemerkten Sie“?

Mit der Erfasssung der individuellen Anzahl der Frühsymptome lassen sich weitere Fragestellungen bezüglich der Prodromalphase dann in zukünftigen Studien untersuchen. Hier wäre zum Beispiel eine Assoziation mit der Dauer der Prodromalphase, dem Leidensdruck und dem Hilfesuchverhalten denkbar. Die Anzahl der Frühsymtpome spielt mit hoher Wahrscheinlichkeit eine Rolle in der diagnostischen Einordung des Frühstadiums der Depression und sollte daher genauer erfasst und im freien Abruf von den Probanden ohne Einschränkung erfolgen.

Hilfesuchverhalten und Beeinträchtigung der Funktionaliät. Im Hinblick auf die genderspezifischen Unterschiede in der Dauer der Prodromalphase sollten weitere Informationen, wie zum Beispiel der subjektiv empfundene Leidensdruck im Zusammenhang mit den Prodromalsymptomen und die Gründe für ein verzögertes Hilfesuchverhalten erhoben werden. Möglicherweise gibt es hier genderspezifische Unterschiede in der wahrgenommenen Beeinträchtigung der Funktionaliät und dem Leidensdruck beim Erleben von Prodromalsymptomen.

Erfassung klinischer Charakteristika der Erstmanifestation. Im Rahmen der Pilotstudie wurde anhand des DEEP-Interviews die zeitliche Verortung der depressiven Erstmanifestation bei Patienten retrospektiv erfasst. Für Patienten mit rezidivierenden Verläufen wurde die Dauer der Prodromalphase oder der Zeitraum bis zum Erstkontakt mit einer medizinisch-psychiatrischen Einrichtung im Rahmen der Erstmanifestation jedoch nicht weiter exploriert. Das DEEP-Interview bezieht sich aktuell ausschließlich auf die zeitlich jüngste und die mnestisch repräsentativste Episode. Dies kann dann gegebenenfalls entweder eine klinische Erstmanifestation oder eine Episode im Rahmen eines rezidivierenden Verlaufs sein. Aus einer differenzierten Erhebung der Charakteristika der Erstmanifestation im Falle eines rezidivierenden Verlaufs würden sich jedoch weitere wichtige Informationen zur Ätiopathogenese der Erkrankung ergeben. Daher empfiehlt sich eine Ergänzung des DEEP-Interviews um die zeitlichen Abläufe und klinischen Charakteristika 
der Erstmanifestation, sofern der zu untersuchende Proband nicht im Rahmen einer Erstmanifestation zur Untersuchung vorstellig ist, das heißt einen rezidivierenden Verlauf aufweist. Ein besonderes Augenmerk sollte auch hier auf der Prodromalphase und der Dauer der unbehandelten Erkrankung im Rahmen der Erstmanifestation liegen.

Ersterkrankungsalter. Eine weitere Optimierungsmöglichkeit wäre die detailliertere Erfassung des Ersterkrankungsalters. Das vorliegende Item erfragt in einem ersten Schritt den Zeitraum der ersten depressiven Episode und in einem zweiten Schritt die Diagnosestellung. Wie bereits erwähnt unterliegen retrospektive Berichte häufig Erinnerungsfehlern und denkbar ist hier ein Nichterinnern vor allem von milderen Episoden, welche nicht diagnostiziert wurden. Um dieser Verzerrung entgegenzuwirken wäre eine dritte Stufe für das genannte Item sinnvoll, welche im Falle einer berichteten diagnostizierten Erstepisode nach früheren nicht diagnostizierten Erkrankungsphasen fragt. Eine Abklärung der Symptomschwere und der Erfüllung diagnostischer Kriterien sollte ebenfalls an dieser Stelle ergänzt werden. Nachgeschaltet werden sollte auch ein Item zur Abklärung einer erfolgten Behandlung im Rahmen der Erstepisode.

Weitere Variablen. Das DEEP-Inventar sollte um eine weitere Variable ergänzt werden. Eine differenzierte Erfassung des Familienklimas im Rahmen der Prodromalphase sollte beispielsweise entweder in das DEEP-Interview oder den DEEP-C integriert werden. Dieser Faktor wird bislang nur indirekt über Fragen zur Lebensqualität und sozialen Unterstützung erfasst. Dies könnte weiteren Aufschluss über die Ätiogenese der Depression geben.

\subsection{Implikationen}

Für die klinische Praxis und die Früherkennungsforschung lassen sich aus der hier dargestellten Modell- und Messinstrumentsentwicklung sowie aus den vorliegenden Daten der Pilotstudie folgende Implikationen ableiten: Eine umfangreiche Erhebung von soziodemographischen und anamnestischen Daten sollte Teil eines Früherkennungsinstruments sein. Aus den daraus gewonnen Daten lassen sich wertvolle Parameter zur Risikokalkulation depressiver Erkrankungen generieren. Die psychiatrische Familienanamnese, Persönlichkeitsmerkmale und das Ersterkrankungsalter einer 
depressiven Störung bilden hilfreiche Faktoren in der Abschätzung des Erkrankungsrisikos. Die Erfassung, Analyse und Klassifikation der Prodromalsymptome ist ein weiterer zentraler Aspekt in der Prävention der Depression und unverzichtbarer Teil eines Früherkennungsinventars. Die dargestellte Länge der Prodromalphase spricht für einen schleichenden Beginn der Depression, woraus sich die Möglichkeit für präventive Maßnahmen vor Ausbruch der Erkrankung eröffnet. Dieses Zeitfenster kann von den Versorgungsstrukturen und insbesondere auch von den Betroffenen selbst genutzt werden, um den Schweregrad einer Erkrankung abzumildern oder deren Ausbruch gänzlich zu verhindern. Ein entsprechendes Hilfesuchverhalten kann jedoch nur gelingen, wenn die Symptomatik von den Betroffenen und den Behandlern rechtzeitig erkannt und richtig zugeordnet werden kann und geeignete Anlaufstellen für unterstützende Maßnahmen zur Verfügung stehen. Eine umfassendere gesundheitliche Aufklärung über die Frühsymptome der Depression und die klinischen Charakteristika der Prodromalphase sind daraus gefordert. Die Kenntnis der individuellen Frühwarnzeichen als auch eine Schulung der metakognitiven Fähigkeiten zur Wahrnehmung und Einordnung dieser Symptome sollten Teil individualisierter Präventivprogramme sein. Die Früherkennung der Depression muss daher nicht nur symptomorientiert gestaltet werden, sondern auf einer metakognitiven Ebene auch die Wahrnehmung für die individuellen Frühwarnzeichen der Depression bei den Hochrisiko-Personen stärken.

\subsection{Fazit und Ausblick}

Im Allgemeinen muss von alters- und genderspezifischen Risiko- und Schutzparametern ausgegangen werden sowie von differentiellen ätiopathogenetischen Mechanismen depressiver Erkrankungen für die Erstmanifestation und rezidivierende Verläufe. Wie auch von Hammen (2015) postuliert, muss die Modellbildung zur Entstehung der Depression bidirektionale Zusammenhänge berücksichtigen. Statische Modelle müssen von dynamischen und interindividuell angepassten Erklärungsmodellen abgelöst werden. Das vorliegende Forschungsvorhaben wurde konzipiert, um diesen Forderungen gerecht zu werden. Die Annäherung an eine erschöpfende Erfassung aller relevanten Faktoren ist ein Vorhaben, welches den Grundstein für weitere Forschung in der Früherkennung der Depression legt. Eine umfassende und erschöpfende Integration aller zu berücksichtigenden 
Risikofaktoren kann dabei aufgrund der Komplexität der Zusammenhänge mit hoher Wahrscheinlichkeit nicht gelingen. Nach der Identifikation und empirischen Prüfung der angenommen Pfade muss die Umsetzung in mathematische Modelle und die Überprüfung mit geeigneten Methoden ein Ziel zukünftiger Forschung sein.

In der vorliegenden Arbeit wurden die Prodromalphase der Depression und einzelne spezifische Risikofaktoren der Ätiogenese untersucht. Weitere im dargestellten Entstehungsmodell der Depression integrierte Risiko- und Schutzfaktoren und deren Einfluss auf das Erkrankungsrisiko werden an anderer Stelle veröffentlicht. Von besonderem Interesse werden dabei kritische Lebensereignisse und deren Interaktion mit Persönlichkeitsmerkmalen sowie Ressourcen und Resilienzfaktoren sein.

Letztlich liegt das übergeordnete Ziel in der Etablierung von individualisierten und ökonomsichen Präventivmaßnahmen zur Reduktion von Inzidenz- und Prävalenzraten. Dies kann nur bei Vorliegen geeigneter Messinstrumente sowie einer Operationalisierung des Prodroms der Depression gelingen. Die vorliegende Studie bildet einen ersten Schritt in diese Richtung. Zukünftige Forschungsvorhaben im Rahmen dieses Projekts werden die Weiterentwicklung des Messinstruments sowie dessen Validierung und Nutzbarkeit in der Risikokalkulation überprüfen. Sofern dies gelingt, werden spezifische Interventionsmaßnahmen zu generieren sein, wobei auch hier die multiplen Einflussfaktoren der Wirksamkeit von Präventivmaßnahmen zu eruieren sein werden. Die Bildung multifaktorieller Wirksamkeitsmodelle, welche unterschiedliche psychopathologische Aspekte der Depression berücksichtigen, wird sich daran anschließen. Eine Integration neurobiologischer, endokriner, physikalischer und immunologischer Aspekte muss dabei erfolgen und ist Ziel dieses Forschungsvorhabens. 


\section{Literatur}

Addington, J., Liu, L., Buchy, L., Cadenhead, K. S., Cannon, T. D., Cornblatt, B. A.,... McGlashan, T. H. (2015). North American Prodrome Longitudinal Study (NAPLS 2): The Prodromal Symptoms. The Journal of Nervous and Mental Disease, 203(5), 328-335. doi:10.1097/NMD.0000000000000290

Akhtar-Danesh, N., \& Landeen, J. (2007). Relation between depression and sociodemographic factors. International Journal of Mental Health Systems, 1(1), 4. doi:10.1186/1752-4458-1-4

Akiskal, H. S., \& McKinney, W. T. (1975). Overview of recent research in depression: Integration of ten conceptual models into a comprehensive clinical frame. Archives of General Psychiatry, 32(3), 285-305. doi:10.1001/archpsyc.1975.01760210019001

Alvaro, P. K., Roberts, R. M., \& Harris, J. K. (2013). A Systematic Review Assessing Bidirectionality between Sleep Disturbances, Anxiety, and Depression. Sleep, 36(7), 10591068. doi:10.5665/sleep. 2810

Andrade, L., Caraveo-anduaga, J. J., Berglund, P., Bijl, R. V., Graaf, R. de, Vollebergh, W.,. . . Wittchen, H.-U. (2003). The epidemiology of major depressive episodes: Results from the International Consortium of Psychiatric Epidemiology (ICPE) surveys. International Journal of Methods in Psychiatric Research, 12(1), 3-21. doi:10.1002/mpr.138

Angermeyer, M. C. (2003). The stigma of mental illness from the patient's view--an overview [Das Stigma psychischer Krankheit aus der Sicht der Patienten - Ein Uberblick]. Psychiatrische Praxis, 30(7), 358-366. doi:10.1055/s-2003-43243

Avorn, J., Everitt, D. E., \& Weiss, S. (1986). Increased antidepressant use in patients prescribed beta-blockers. The Journal of the American Medical Association, 255(3), 357360.

Barlow, D. H., Ellard, K. K., Sauer-Zavala, S., Bullis, J. R., \& Carl, J. R. (2014). The Origins of Neuroticism. Perspectives on Psychological Science : A Journal of the Association for Psychological Science, 9(5), 481-496. doi:10.1177/1745691614544528

Baron, Mendlewicz \& Klotz. (1981). Age-of-onset and genetic transmission in affective disorders. Acta Psychiatrica Scandinavica. (64.5), 373-380.

Beesdo, K., Höfler, M., Leibenluft, E., Lieb, R., Bauer, M., \& Pfennig, A. (2009). Mood episodes and mood disorders: patterns of incidence and conversion in the first three decades of life. Bipolar Disorders, 11(6), 637-649. doi:10.1111/j.1399-5618.2009.00738.x

Beesdo-Baum, K., \& Wittchen, H.-U. (2011). Klinische Psychologie \& Psychotherapie (2., überarb. und erw. Aufl.). Heidelberg: Springer-Medizin.

Bellón, J. Á., Dios Luna, J. de, King, M., Moreno-Küstner, B., Nazareth, I., Montón-Franco, C.,... Torres-González, F. (2011). Predicting the onset of major depression in primary care: international validation of a risk prediction algorithm from Spain. Psychological Medicine, 41(10), 2075-2088. doi:10.1017/S0033291711000468 
Ben-Zeev, D., Young, M. A., \& Madsen, J. W. (2009). Retrospective recall of affect in clinically depressed individuals and controls. Cognition and Emotion, 23(5), 1021-1040. doi:10.1080/02699930802607937

Bernet, C. Z., \& Stein, M. B. (1999). Relationship of childhood maltreatment to the onset and course of major depression in adulthood. Depression and Anxiety, 9(4), 169-174.

Blanz, B. (2006). Psychische Störungen im Kindes- und Jugendalter: Ein entwicklungspsychopathologisches Lehrbuch. Stuttgart: Schattauer.

Brewin, C. R., Andrews, B., \& Gotlib, I. H. (1993). Psychopathology and early experience: A reappraisal of retrospective reports. Psychological Bulletin, 113(1), 82-98. doi:10.1037/0033-2909.113.1.82

Bulloch, A., Williams, J., Lavorato, D., \& Patten, S. (2014). Recurrence of major depressive episodes is strongly dependent on the number of previous episodes. Depression and Anxiety, 31(1), 72-76. doi:10.1002/da.22173

Burcusa, S. L., \& lacono, W. G. (2007). Risk for recurrence in depression. Clinical Psychology Review, 27(8), 959-985. doi:10.1016/j.cpr.2007.02.005

Buysse, D. J., Angst, J., Gamma, A., Ajdacic, V., Eich, D., \& Rössler, W. (2007). Prevalence, Course, and Comorbidity of Insomnia and Depression in Young Adults. Sleep, 31(4), 473480.

Cairns, K. E., Yap, M. B., Pilkington, P. D., \& Jorm, A. F. (2014). Risk and protective factors for depression that adolescents can modify: A systematic review and meta-analysis of longitudinal studies. Journal of Affective Disorders, 169, 61-75.

Carr, C. P., Martins, C. M. S., Stingel, A. M., Lemgruber, V. B., \& Juruena, M. F. (2013). The role of early life stress in adult psychiatric disorders: A systematic review according to childhood trauma subtypes. Journal of Nervous and Mental Disease, 201(12), 1007-1020. doi:10.1097/NMD.0000000000000049

Clark, L. A., Watson, D., \& Mineka, S. (1994). Temperament, personality, and the mood and anxiety disorders. Journal of Abnormal Psychology, 103(1), 103-116. doi:10.1037/0021843X.103.1.103

Curry, J., Silva, S., Rohde, P., Ginsburg, G., Kratochvil, C., Simons, A.,. . March, J. (2011). Recovery and recurrence following treatment for adolescent major depression. Archives of General Psychiatry, 68(3), 263-269. doi:10.1001/archgenpsychiatry.2010.150

Dell'Osso, B., \& Altamura, A. C. (2010). Duration of Untreated Psychosis and Duration of Untreated Illness: New Vistas. CNS Spectrums, 15(4), 238-246. doi:10.1017/S1092852900000079

Dilling, H., Mombour, W., \& Schmidt, M. H. (Eds.). (2014). Internationale Klassifikation psychischer Störungen: ICD-10 Kapitel $V$ (F) Klinisch diagnostische Leitlinien (9th ed.). Bern: Hans Huber.

Donini, L. M., Savina, C., \& Cannella, C. (1999). Eating Habits and Appetite Control in the Elderly: The Anorexia of Aging. International Psychogeriatrics, 15(1), 73-87. doi:10.1017/S1041610203008779 
Eaton, W. W., Badawi, M., \& Melton, B. (1995). Prodromes and precursors: epidemiologic data for primary prevention of disorders with slow onset. The American Journal of Psychiatry, 152(7), 967-972. doi:10.1176/ajp.152.7.967

Eaton, W. W., Shao, H., Nestadt, G., Lee, H. B., Bienvenu, O. J., \& Zandi, P. (2008). Population-based study of first onset and chronicity in major depressive disorder. Archives of General Psychiatry, 65(5), 513-520. doi:10.1001/archpsyc.65.5.513

Falkai, P., \& Wittchen, H.-U. (Eds.). (2015). Diagnostisches und Statistisches Manual Psychischer Störungen DSM-5. Göttingen: Hogrefe.

Farb, N. A., Anderson, A. K., Bloch, R. T., \& Segal, Z. V. (2011). Mood-Linked Responses in Medial Prefrontal Cortex Predict Relapse in Patients with Recurrent Unipolar Depression. Biological Psychiatry, 70(4), 366-372. doi:10.1016/j.biopsych.2011.03.009

Faul, F., Erdfelder, E., Buchner, A., \& Lang, A.-G. (2009). Statistical power analyses using G*Power 3.1: tests for correlation and regression analyses. Behavior Research Methods, 41(4), 1149-1160. doi:10.3758/BRM.41.4.1149

Fava, G. A., Grandi, S., Zielezny, M., Canestrari, R., \& Morphy, M. A. (1994). Cognitive behavioral treatment of residual symptoms in primary major depressive disorder. The American Journal of Psychiatry, 151(9), 1295-1299. doi:10.1176/ajp.151.9.1295

Fava, G. A., \& Kellner, R. (1991). Prodromal symptoms in affective disorders. The American Journal of Psychiatry, 148(7), 823-830. doi:10.1176/ajp.148.7.823

Fava, G. A., Grandi, S., Canestrari, R., \& Molnar, G. (1990). Prodromal symptoms in primary major depressive disorder. Journal of Affective Disorders, 19(2), 149-152. doi:10.1016/0165-0327(90)90020-9

Fava, G. A., \& Tossani, E. (2007). Prodromal stage of major depression. Early Intervention in Psychiatry, 1(1), 9-18. doi:10.1111/j.1751-7893.2007.00005.x

Ferrari, A. J., Charlson, F. J., Norman, R. E., Patten, S. B., Freedman, G., Murray, C. J. L.,. . . Whiteford, H. A. (2013). Burden of depressive disorders by country, sex, age, and year: findings from the global burden of disease study 2010. PLoS Medicine, 10(11), e1001547. doi:10.1371/journal.pmed.1001547

Ge, X., Conger, R. D., \& Elder, G. H. (2001). Pubertal transition, stressful life events, and the emergence of gender differences in adolescent depressive symptoms. Developmental Psychology, 37(3), 404-417. doi:10.1037/0012-1649.37.3.404

Georgiades, K., Lewinsohn, P. M., Monroe, S. M., \& Seeley, J. R. (2006). Major depressive disorder in adolescence: the role of subthreshold symptoms. Journal of the American Academy of Child and Adolescent Psychiatry, 45(8), 936-944. doi:10.1097/01.chi.0000223313.25536.47

Ghio, L., Gotelli, S., Cervetti, A., Respino, M., Natta, W., Marcenaro, M.,. . Belvederi Murri, M. (2015). Duration of untreated depression influences clinical outcomes and disability. Journal of Affective Disorders, 175, 224-228. doi:10.1016/j.jad.2015.01.014 
Grav, S., Stordal, E., Romild, U. K., \& Hellzen, O. (2012). The relationship among neuroticism, extraversion, and depression in the HUNT Study: in relation to age and gender. Issues in Mental Health Nursing, 33(11), 777-785. doi:10.3109/01612840.2012.713082

Grayson, L., \& Thomas, A. (2013). A systematic review comparing clinical features in early age at onset and late age at onset late-life depression. Journal of Affective Disorders, 150(2), 161-170. doi:10.1016/j.jad.2013.03.021

Hamdi, N. R., \& lacono, W. G. (2014). Lifetime prevalence and co-morbidity of externalizing disorders and depression in prospective assessment. Psychological Medicine, 44(02), 315324. doi:10.1017/S0033291713000627

Hamilton, J. P., Etkin, A., Furman, D. J., Lemus, M. G., Johnson, R. F., \& Gotlib, I. H. (2012). Functional neuroimaging of major depressive disorder: a meta-analysis and new integration of base line activation and neural response data. The American Journal of Psychiatry, 169(7), 693-703. doi:10.1176/appi.ajp.2012.11071105

Hammen, C. L. (2015). Stress and depression: Old questions, new approaches. Current Opinion in Psychology, 4, 80-85. doi:10.1016/j.copsyc.2014.12.024

Hankin, B. L., Abramson, L. Y., Moffitt, T. E., Silva, P. A., McGee, R., \& Angell, K. E. (1998). Development of depression from preadolescence to young adulthood: Emerging gender differences in a 10-year longitudinal study. Journal of Abnormal Psychology, 107(1), 128140. doi:10.1037/0021-843X.107.1.128

Hardeveld, F., Spijker, J., Graaf, R. de, Nolen, W. A., \& Beekman, A. T. F. (2010). Prevalence and predictors of recurrence of major depressive disorder in the adult population. Acta Psychiatrica Scandinavica, 122(3), 184-191. doi:10.1111/j.1600-0447.2009.01519.x

Hardeveld, F., Spijker, J., Graaf, R. de, Nolen, W. A., \& Beekman, A. T. F. (2013). Recurrence of major depressive disorder and its predictors in the general population: results from the Netherlands Mental Health Survey and Incidence Study (NEMESIS). Psychological Medicine, 43(1), 39-48. doi:10.1017/S0033291712002395

Hargus, E., Crane, C., Barnhofer, T., \& Williams, J. M. G. (2010). Effects of mindfulness on meta-awareness and specificity of describing prodromal symptoms in suicidal depression. Emotion, 10(1), 34-42. doi:10.1037/a0016825

Hautzinger, M. (1997). Kognitive Verhaltenstherapie bei Depressionen im Alter. In H. Radebold, R. D. Hirsch, J. Kipp, R. Kortus, G. Stoppe, B. Struwe, \& C. Wächtler (Eds.), Depressionen im Alter (pp. 60-68). Heidelberg: Steinkopff. doi:10.1007/978-3-642-854408_7

Hautzinger, M. (2013). Kognitive Verhaltenstherapie bei Depressionen. Bern: Beltz.

Hautzinger, M. (2015a). Depressionen. In M. Hautzinger \& M. Linden (Eds.), Verhaltenstherapiemanual (pp. 511-516). Berlin-Heidelberg: Springer.

Hautzinger, M. (2015b). Depressive Störungen. In A. Maercker (Ed.), Alterspsychotherapie und klinische Gerontopsychologie (pp.119-137). Berlin, Heidelberg: Springer. doi:10.1007/978-3-642-54723-2_6 
Hayes, A. F. (2013). Introduction to mediation, moderation, and conditional process analysis: A regression-based approach. Methodology in the social sciences. New York: The Guilford Press. Retrieved from http://www.personal.psu.edu/jxb14/M554/specreg/templates.pdf Hegeman, J. M., Kok, R. M., van der Mast, R. C., \& Giltay, E. J. (2012). Phenomenology of depression in older compared with younger adults: meta-analysis. British Journal of Psychiatry, 200(4), 275. doi:10.1192/bjp.bp.111.095950

Hegerl, U., Rummel-Kluge, C., \& Heinz, I. (2016). From the Competence Network on Depression and Suicidality to the German Depression Foundation. Bundesgesundheitsblatt - Gesundheitsforschung - Gesundheitsschutz, 59(4), 406-411. doi:10.1007/s00103-016-2310-2

Henry, B., Moffitt, T. E., Caspi, A., Langley, J., \& Silva, P.A. (1994). On the "remembrance of things past": A longitudinal evaluation of the retrospective method. Psychological Assessment, 6(2), 92-101. doi:10.1037/1040-3590.6.2.92

Heser, K., Tebarth, F., Wiese, B., Eisele, M., Bickel, H., Kohler, M.,. . Wagner, M. (2013). Age of major depression onset, depressive symptoms, and risk for subsequent dementia: results of the German study on Ageing, Cognition, and Dementia in Primary Care Patients (AgeCoDe). Psychological Medicine, 43(8), 1597-1610. doi:10.1017/S0033291712002449

Hetrick, S. E., Parker, A. G., Hickie, I. B., Purcell, R., Yung, A. R., \& McGorry, P. D. (2008). Early identification and intervention in depressive disorders: towards a clinical staging model. Psychotherapy and Psychosomatics, 77(5), 263-270. doi:10.1159/000140085

Hidaka, B. H. (2013). Depression as a disease of modernity: Explanations for increasing prevalence. Journal of Affective Disorders, 140(3), 205-214. doi:10.1016/j.jad.2011.12.036

Hölzel, L., Härter, M., Reese, C., \& Kriston, L. (2011). Risk factors for chronic depression - A systematic review. Journal of Affective Disorders, 129(1), 1-13. doi:10.1016/j.jad.2010.03.025

lacoviello, B. M., Alloy, L. B., Abramson, L. Y., \& Choi, J. Y. (2010). The early course of depression: A longitudinal investigation of prodromal symptoms and their relation to the symptomatic course of depressive episodes. Journal of Abnormal Psychology, 119(3), 459-467. doi:10.1037/a0020114

Jackson, A., Cavanagh, J., \& Scott, J. (2003). A systematic review of manic and depressive prodromes. Journal of Affective Disorders, 74(3), 209-217.

Jacobi, F., \& Kessler-Scheil, S. (2013). Epidemiologie psychischer Störungen: Häufigkeit und Krankheitslast in Deutschland. Psychotherapeut, 58(2), 191-206.

Jacobi, F., Wittchen, H.-U., Holting, C., Hofler, M., Pfister, H., Muller, N., \& Lieb, R. (2004). Prevalence, co-morbidity and correlates of mental disorders in the general population: results from the German Health Interview and Examination Survey (GHS). Psychological Medicine, 34(4), 597-611. doi:10.1017/S0033291703001399

Jacobi, F., Höfler, M., Strehle, J., Mack, S., Gerschler, A., Scholl, L.,. . W Wittchen, H. (2015). Twelve-months prevalence of mental disorders in the German Health Interview and 
Examination Survey for Adults - Mental Health Module (DEGS1-MH): a methodological addendum and correction. International Journal of Methods in Psychiatric Research, 24(4), 305-313. doi:10.1002/mpr.1479

Kaluza, G. (2015). Stressbewältigung: Trainingsmanual zur psychologischen Gesundheitsförderung. Psychotherapie Praxis. Berlin: Springer.

Keenan, K., Hipwell, A., Feng, X., Babinski, D., Hinze, A., Rischall, M., \& Henneberger, A. (2008). Subthreshold symptoms of depression in preadolescent girls are stable and predictive of depressive disorders. Journal of the American Academy of Child and Adolescent Psychiatry, 47(12), 1433-1442. doi:10.1097/CHI.0b013e3181886eab

Kendler, K. S., Karkowski, L. M., \& Prescott, C. A. (1999). Causal relationship between stressful life events and the onset of major depression. The American Journal of Psychiatry, 156(6), 837-841. doi:10.1176/ajp.156.6.837

Kendler, K. S., Prescott, C. A., Myers, J., \& Neale, M. C. (2003). The structure of genetic and environmental risk factors for common psychiatric and substance use disorders in men and women. Archives of General Psychiatry, 60(9), 929-937. doi:10.1001/archpsyc.60.9.929

Kendler, K. S., \& Gardner, C. O. (2014). Sex differences in the pathways to major depression: a study of opposite-sex twin pairs. The American Journal of Psychiatry, 171(4), 426-435. doi:10.1176/appi.ajp.2013.13101375

Kendler, K. S., Gardner, C. O., \& Prescott, C. A. (2002). Toward a comprehensive developmental model for major depression in women. The American Journal of Psychiatry, 159(7), 1133-1145. doi:10.1176/appi.ajp.159.7.1133

Kendler, K. S., Gatz, M., Gardner, C. O., \& Pederse, N. L. (2006). Personality and Major Depression. Archives of General Psychiatry, 63(10), 1113-1120. doi:10.1001/archpsyc.63.10.1113

Kendler, K. S., Kuhn, J. W., \& Prescott, C. A. (2004). Childhood sexual abuse, stressful life events and risk for major depression in women. Psychological Medicine, 34(8), 14751482.

Kendler, K. S., Thornton, L. M., \& Gardner, C. O. (2000). Stressful life events and previous episodes in the etiology of major depression in women: An evaluation of the 'Kindling' hypothesis. The American Journal of Psychiatry, 157(8), 1243-1251. doi:10.1176/appi.ajp.157.8.1243

Kern, N., Sheldrick, A. J., Schmidt, F. M., \& Minkwitz, J. (2012). Neurobiology of depression and novel antidepressant drug targets. Current Pharmaceutical Design, 18(36), 57915801.

Kessler, R. (2003). Epidemiology of women and depression. Journal of Affective Disorders, 74(1), 5-13. doi:10.1016/S0165-0327(02)00426-3

Kessler, R. C., van Loo, H. M., Wardenaar, K. J., Bossarte, R. M., Brenner, L. A., Cai, T.,... Zaslavsky, A. M. (2016). Testing a machine-learning algorithm to predict the persistence 
and severity of major depressive disorder from baseline self-reports. Molecular Psychiatry, 21, 1366-1371.

Kessler, R. C. (2012). The Costs of Depression. Psychiatric Clinics of North America, 35(1), 114. doi:10.1016/j.psc.2011.11.005

Kessler, R. C., Berglund, P., Demler, O., Jin, R., Merikangas, K. R., \& Walters, E. E. (2005). Lifetime prevalence and age-of-onset distributions of DSM-IV disorders in the National Comorbidity Survey Replication. Archives of General Psychiatry, 62(6), 593-602. doi:10.1001/archpsyc.62.6.593

Kessler, R. C., Birnbaum, H., Bromet, E., Hwang, I., Sampson, N., \& Shahly, V. (2009). Age Differences in Major depression: Results from the National Comorbidity Surveys Replication (NCS-R). Psychological Medicine, 40(2), 225. doi:10.1017/S0033291709990213

Kessler, R. C., Birnbaum, H., Shahly, V., Bromet, E., Hwang, I., McLaughlin, K. A.,. . Stein, D. J. (2010). Age differences in the prevalence and comorbidity of DSM-IV major depressive episodes: Results from the WHO World Mental Health Survey Initiative. Depression and Anxiety, 27(4), 351-364. doi:10.1002/da.20634

Kessler, R. C., \& Bromet, E. J. (2013). The epidemiology of depression across cultures. Annual Review of Public Health, 34, 119-138. doi:10.1146/annurev-publhealth-031912-114409

King, M., Walker, C., Levy, G., Bottomley, C., Royston, P., Weich, S.,. . Nazareth, I. (2008). Development and validation of an international risk prediction algorithm for episodes of major depression in general practice attendees: the PredictD study. Archives of General Psychiatry, 65(12), 1368-1376. doi:10.1001/archpsyc.65.12.1368

Kivelitz, L., Watzke, B., Schulz, H., Harter, M., \& Melchior, H. (2015). Health care barriers on the pathways of patients with anxiety and depressive disorders - a qualitative interview study [Versorgungsbarrieren auf den Behandlungswegen von Patienten mit Angst- und depressiven Erkrankungen - Eine qualitative Interviewstudie]. Psychiatrische Praxis, 42(8), 424-429. doi:10.1055/s-0034-1370306

Klasen, F., Otto, C., Kriston, L., Patalay, P., Schlack, R., \& Ravens-Sieberer, U. (2015). Risk and protective factors for the development of depressive symptoms in children and adolescents: results of the longitudinal BELLA study. European Child \& Adolescent Psychiatry, 24(6), 695-703. doi:10.1007/s00787-014-0637-5

Knäuper, B., \& Wittchen, H.-U. (1994). Diagnosing major depression in the elderly: Evidence for response bias in standardized diagnostic interviews? Journal of Psychiatric Research, 28(2), 147-164. doi:10.1016/0022-3956(94)90026-4

Korten, N. C., Comijs, H. C., Lamers, F., \& Penninx, B. W. (2012). Early and late onset depression in young and middle aged adults: Differential symptomatology, characteristics and risk factors? Journal of Affective Disorders, 138(3), 259-267. doi:10.1016/j.jad.2012.01.042

Kotov, R., Gamez, W., Schmidt, F., \& Watson, D. (2010). Linking "big" personality traits to anxiety, depressive, and substance use disorders: a meta-analysis. Psychological Bulletin, 136(5), 768-821. doi:10.1037/a0020327 
Koutsouleris, N., Riecher-Rossler, A., Meisenzahl, E. M., Smieskova, R., Studerus, E., Kambeitz-Ilankovic, L.,. . Borgwardt, S. (2015). Detecting the psychosis prodrome across high-risk populations using neuroanatomical biomarkers. Schizophrenia Bulletin, 41(2), 471-482. doi:10.1093/schbul/sbu078

Kovacs, M., \& Lopez-Duran, N. (2010). Prodromal symptoms and atypical affectivity as predictors of major depression in juveniles: implications for prevention. Journal of Child Psychology and Psychiatry, and Allied Disciplines, 51(4), 472-496. doi:10.1111/j.14697610.2010.02230.x

Kraemer, H. C., Stice, E., Kazdin, A., Offord, D., \& Kupfer, D. (2001). How do risk factors work together? Mediators, moderators, and independent, overlapping, and proxy risk factors. The American Journal of Psychiatry, 158(6), 848-856. doi:10.1176/appi.ajp.158.6.848

Lieb, R., Isensee, B., Höfler, M., Pfister, H., \& Wittchen, H. (2002). Parental major depression and the risk of depression and other mental disorders in offspring: A prospectivelongitudinal community study. Archives of General Psychiatry, 59(4), 365-374. doi:10.1001/archpsyc.59.4.365

Lindert, J., Ehrenstein, O. S. von, Grashow, R., Gal, G., Braehler, E., \& Weisskopf, M. G. (2014). Sexual and physical abuse in childhood is associated with depression and anxiety over the life course: Systematic review and meta-analysis. International Journal of Public Health, 59(2), 359-372. doi:10.1007/s00038-013-0519-5

Lyketsos, C. G., Nestadt, G., Cwi, J., Heithoff, K., \& et al. (1994). The Life Chart Interview: A standardized method to describe the course of psychopathology. International Journal of Methods in Psychiatric Research, 4, 143-155.

Mahnert, F. A., Reicher, H., Zalaudek, K., \& Zapotoczky, H. G. (1997). Prodromal and residual symptoms in recurrent depression: Preliminary data of a long-term study under prophylactic treatment condition. European Neuropsychopharmacology, 7, 159-160. doi:10.1016/S0924-977X(97)88527-6

Malouff, J. M., Thorsteinsson, E. B., \& Schutte, N. S. (2005). The Relationship Between the Five-Factor Model of Personality and Symptoms of Clinical Disorders: A Meta-Analysis. Journal of Psychopathology and Behavioral Assessment, 27(2), 101-114. doi:10.1007/s10862-005-5384-y

Mandelli, L., Petrelli, C., \& Serretti, A. (2015). The role of specific early trauma in adult depression: A meta-analysis of published literature. Childhood trauma and adult depression. The Journal of the Association of European Psychiatrists, 30(6), 665-680. doi:10.1016/j.eurpsy.2015.04.007

Margraf, J. \& Schneider, S. (Ed.). (2009). Lehrbuch der Verhaltenstherapie (3rd ed., Vol. 2). Heidelberg: Springer.

Martin, L. A., Neighbors, H. W., \& Griffith, D. M. (2013). The experience of symptoms of depression in men vs women: Analysis of the national comorbidity survey replication. The Journal of the American Medical Association, 70(10), 1100-1106. doi:10.1001/jamapsychiatry.2013.1985 
Masten, A. S., Cutuli, J. J., Herbers, J. E., \& Reed, M.-G. (2009). Resilience in development. In C. R. Snyder \& S. J. Lopez (Eds.), The handbook of positive psychology (2nd ed.). New York: Oxford University Press.

Merikangas, K. R., Risch, N. J., \& Weissman, M. M. (1994). Comorbidity and co-transmission of alcoholism, anxiety and depression. Psychological Medicine, 24(01), 69. doi:10.1017/S0033291700026842

Merikangas, K. R., Chakravarti, A., Moldin, S. O., Araj, H., Blangero, J. C., Burmeister, M.,. . . Takahashi, J. S. (2002). Future of genetics of mood disorders research. Biological Psychiatry, 52(6), 457-477.

Mitchell, A. J., Vaze, A., \& Rao, S. (2009). Clinical diagnosis of depression in primary care: A meta-analysis. The Lancet, 374(9690), 609-619. doi:10.1016/S0140-6736(09)60879-5

Moffitt, T. E., Caspi, A., Taylor, A., Kokaua, J., Milne, B. J., Polanczyk, G., \& Poulton, R. (2010). How common are common mental disorders?: Evidence that lifetime prevalence rates are doubled by prospective versus retrospective ascertainment. Psychological Medicine, 40(06), 899-909. doi:10.1017/S0033291709991036

Möller-Leimkühler, A. M. (2000). Men and depression: gender-related help-seeking behavior [Manner und Depression: geschlechtsspezifisches Hilfesuchverhalten]. Fortschritte der Neurologie-Psychiatrie, 68(11), 489-495. doi:10.1055/s-2000-10030

Möller-Leimkühler, A. M. (2008). Depression - überdiagnostiziert bei Frauen, unterdiagnostiziert bei Männern? Der Gynäkologe, 41(5), 381-388. doi:10.1007/s00129008-2161-5

Monroe, S. M., Slavich, G. M., \& Gotlib, I. H. (2014). Life stress and family history for depression: the moderating role of past depressive episodes. Journal of Psychiatric Research, 49, 90-95. doi:10.1016/j.jpsychires.2013.11.005

Monteiro, S., Fernandes da Silva, C., Pereira, A., \& Vaz Serra, A. (2011). Risk factors for depression in young adulthood: pessimism, dysfunctional attitudes and neuroticism. European Psychiatry, 26, 654. doi:10.1016/S0924-9338(11)72360-4

Mueller, T. I., Leon, A. C., Keller, M. B., Solomon, D. A., Endicott, J., Coryell, W.,. . Maser, J. D. (1999). Recurrence after recovery from major depressive disorder during 15 years of observational follow-up. The American Journal of Psychiatry, 156(7), 1000-1006. doi:10.1176/ajp.156.7.1000

Naismith, S. L., Norrie, L. M., Mowszowski, L., \& Hickie, I. B. (2012). The neurobiology of depression in later-life: clinical, neuropsychological, neuroimaging and pathophysiological features. Progress in Neurobiology, 98(1), 99-143. doi:10.1016/j.pneurobio.2012.05.009

Nolen-Hoeksema, S. (2000). The role of rumination in depressive disorders and mixed anxiety/depressive symptoms. Journal of Abnormal Psychology, 109(3), 504-511. doi:10.1037/0021-843X.109.3.504

Noteboom, A., Beekman, A. T., Vogelzangs, N., \& Penninx, B. W. (2016). Personality and social support as predictors of first and recurrent episodes of depression. Journal of Affective Disorders, 190, 156-161. doi:10.1016/j.jad.2015.09.020 
Notzon, S., Alferink, J., \& Arolt, V. (2016). Late-onset depression: Pathophysiology, diagnostics and treatment [Altersdepression: Pathophysiologie, Diagnostik und Therapie]. Der Nervenarzt. doi:10.1007/s00115-016-0193-y

Olino, T. M., Klein, D. N., Lewinsohn, P. M., Rohde, P., \& Seeley, J. R. (2010). Latent trajectory classes of depressive and anxiety disorders from adolescence to adulthood: descriptions of classes and associations with risk factors. Comprehensive Psychiatry, 51(3), 224-235. doi:10.1016/j.comppsych.2009.07.002

Olino, T. M., Shankman, S. A., Klein, D. N., Seeley, J. R., Pettit, J. W., Farmer, R. F., \& Lewinsohn, P. M. (2012). Lifetime rates of psychopathology in single versus multiple diagnostic assessments: comparison in a community sample of probands and siblings. Journal of Psychiatric Research, 46(9), 1217-1222. doi:10.1016/j.jpsychires.2012.05.017

Oquendo, M. A., Turret, J., Grunebaum, M. F., Burke, A. K., Poh, E., Stevenson, E.,... Galfalvy, H. (2013). Sex differences in clinical predictors of depression: a prospective study. Journal of Affective Disorders, 150(3), 1179-1183. doi:10.1016/j.jad.2013.05.010

Ormel, J., Jeronimus, B. F., Kotov, R., Riese, H., Bos, E. H., Hankin, B.,. . Oldehinkel, A. J. (2013). Neuroticism and common mental disorders: Meaning and utility of a complex relationship. Clinical Psychology Review, 33(5), 686-697. doi:10.1016/j.cpr.2013.04.003

Patten, S. B. (2013). Recurrence risk in major depression. Depression and Anxiety, 30(1), 1-4. doi:10.1002/da.22030

Patten, S. B., Williams, J. V. A., Lavorato, D. H., Bulloch, A. G. M., D'Arcy, C., \& Streiner, D. L. (2012). Recall of recent and more remote depressive episodes in a prospective cohort study. Social Psychiatry and Psychiatric Epidemiology, 47(5), 691-696. doi:10.1007/s00127-011-0385-5

Paykel, E. S., Prusoff, B. A., \& Tanner, J. (1976). Temporal stability of symptom patterns in depression. The British Journal of Psychiatry : the Journal of Mental Science, 128, 369374.

Paykel, E. S., Brugha, T., \& Fryers, T. (2005). Size and burden of depressive disorders in Europe. European Neuropsychopharmacology : the Journal of the European College of Neuropsychopharmacology, 15(4), 411-423. doi:10.1016/j.euroneuro.2005.04.008

Pettit, J. W., Lewinsohn, P. M., Roberts, R. E., Seeley, J. R., \& Monteith, L. (2009). The longterm course of depression: development of an empirical index and identification of early adult outcomes. Psychological Medicine, 39(03), 403-412. doi:10.1017/S0033291708003851

Pettit, J. W., Hartley, C., Lewinsohn, P. M., Seeley, J. R., \& Klein, D. N. (2013). Is liability to recurrent major depressive disorder present before first episode onset in adolescence or acquired after the initial episode? Journal of Abnormal psychology, 122(2), 353-358. doi:10.1037/a0032655

Piccinelli, M. (2000). Gender differences in depression: Critical review. The British Journal of Psychiatry, 177(6), 486-492. doi:10.1192/bjp.177.6.486 
Pine, D. S., Cohen, P., \& Brook, J. (2001). Adolescent fears as predictors of depression. Biological Psychiatry, 50(9), 721-724. doi:10.1016/S0006-3223(01)01238-0

Reynders, A., Kerkhof, A., Molenberghs, G., \& van Audenhove, C. (2014). Attitudes and stigma in relation to help-seeking intentions for psychological problems in low and high suicide rate regions. Social Psychiatry and Psychiatric Epidemiology, 49(2), 231-239. doi:10.1007/s00127-013-0745-4

Rohde, P., Lewinsohn, P. M., Klein, D. N., Seeley, J. R., \& Gau, J. M. (2013). Key Characteristics of Major Depressive Disorder Occurring in Childhood, Adolescence, Emerging Adulthood, Adulthood. Clinical Psychological Science : A Journal of the Association for Psychological Science, 1(1). doi:10.1177/2167702612457599

Rohde, P., Lewinsohn, P. M., \& Seeley, J. R. (1990). Are people changed by the experience of having an episode of depression?: A further test of the scar hypothesis. Journal of Abnormal psychology, 99(3), 264-271. doi:10.1037/0021-843X.99.3.264

Rost, K., Zhang, M., Fortney, J., Smith, J., Coyne, J., \& Richard Smith, G. (1998). Persistently poor outcomes of undetected major depression in primary care. General Hospital Psychiatry, 20(1), 12-20. doi:10.1016/S0163-8343(97)00095-9

Rutter, M. (2000). Psychosocial influences: critiques, findings, and research needs. Development and Psychopathology, 12(3), 375-405.

Santini, Z. I., Koyanagi, A., Tyrovolas, S., Mason, C., \& Haro, J. M. (2015). The association between social relationships and depression: a systematic review. Journal of Affective Disorders, 175, 53-65. doi:10.1016/j.jad.2014.12.049

Savitz, J., \& Drevets, W. C. (2009). Bipolar and major depressive disorder: neuroimaging the developmental-degenerative divide. Neuroscience and Biobehavioral Reviews, 33(5), 699771. doi:10.1016/j.neubiorev.2009.01.004

Schmidt, M. H., \& Göppel, C. (2008). Risikofaktoren - Coping und Verlaufsprinzipien psychischer Störungen. In B. Herpertz-Dahlmann, F. Resch, M. J. Schulte-Markwort, \& A. Warnke (Eds.), Entwicklungspsychiatrie. Biopsychologische Grundlagen und die Entwicklung psychischer Störungen (2nd ed.). Stuttgart: Schattauer.

Schottke, H., \& Giabbiconi, C.-M. (2015). Post-stroke depression and post-stroke anxiety: prevalence and predictors. International Psychogeriatrics, 27(11), 1805-1812. doi:10.1017/S1041610215000988

Sikorski, C., Luppa, M., König, H.-H., van den Bussche, H., \& Riedel-Heller, S. G. (2012). Does GP training in depression care affect patient outcome? - A systematic review and metaanalysis. BMC Health Services Research, 12, 10. doi:10.1186/1472-6963-12-10

Simon, G. E., \& VonKorff, M. (1995). Recall of psychiatric history in cross-sectional surveys: implications for epidemiologic research. Epidemiologic Reviews, 17(1), 221-227.

Snyder, C. R., \& Lopez, S. J. (Eds.). (2009). The handbook of positive psychology (2nd ed.). New York: Oxford University Press. 
Soderlund, H., Moscovitch, M., Kumar, N., Daskalakis, Z. J., Flint, A., Herrmann, N., \& Levine, B. (2014). Autobiographical episodic memory in major depressive disorder. Journal of Abnormal Psychology, 123(1), 51-60. doi:10.1037/a0035610

Sullivan, P. F., Neale, M. C., \& Kendler, K. S. (2000). Genetic epidemiology of major depression: review and meta-analysis. The American Journal of Psychiatry, 157(10), 15521562. doi:10.1176/appi.ajp.157.10.1552

Syed Sheriff, R. J., McGorry, P. D., Cotton, S., \& Yung, A. R. (2015). A Qualitative Study of the Prodrome to First-Episode Major Depressive Disorder in Adolescents. Psychopathology, 48(3), 153-161.

Thiessen, B. Q., Wallace, S. M., Blackburn, J. L., Wilson, T. W., \& Bergman, U. (1990). Increased prescribing of antidepressants subsequent to beta-blocker therapy. Archives of Internal Medicine, 150, 2286-2290.

Tozzi, F., Prokopenko, I., Perry, J. D., Kennedy, J. L., McCarthy, A. D., Holsboer, F., \& ... \& Muglia, P. (2008). Family history of depression is associated with younger age of onset in patients with recurrent depression. Psychological Medicine. (38(05)), 641-649.

Tröster, H. (2009). Früherkennung im Kindes-und Jugendalter: Strategien bei Entwicklungs-, Lern-und Verhaltensstörungen. Hogrefe Verlag. Göttingen: Hogrefe.

Uhmann, S., \& Wittchen, H.-U. (2010). The timing of depression: an epidemiological perspective. MEDICOGRAPHIA, 32(2).

Valdez, R., Yoon, P. W., Qureshi, N., Green, R. F., \& Khoury, M. J. (2010). Family history in public health practice: a genomic tool for disease prevention and health promotion. Annual Review of Public Health, 31, 69-87. doi:10.1146/annurev.publhealth.012809.103621

Weissman, M. M. (1996). Cross-National Epidemiology of Major Depression and Bipolar Disorder. The Journal of the American Medical Association, 276(4), 293. doi:10.1001/jama.1996.03540040037030

Weissman, M. M., Wickramaratne, P., Gameroff, M. J., Warner, V., Pilowsky, D., Kohad, R. G.,... Talati, A. (2016). Offspring of Depressed Parents: 30 Years Later. The American Journal of Psychiatry, appiajp201615101327. doi:10.1176/appi.ajp.2016.15101327

Wells, J. E., \& Horwood, L. J. (2004). How accurate is recall of key symptoms of depression? A comparison of recall and longitudinal reports. Psychological Medicine, 34(6), 1001-1011.

Whitfield-Gabrieli, S., \& Ford, J. M. (2012). Default mode network activity and connectivity in psychopathology. Annual Review of Clinical Psychology, 8, 49-76. doi:10.1146/annurevclinpsy-032511-143049

Wiersma, J. E., Hovens, J. G. F. M., van Oppen, P., Giltay, E. J., van Schaik, D. J. F., Beekman, A. T. F., \& Penninx, B. W. J. H. (2009). The Importance of Childhood Trauma and Childhood Life Events for Chronicity of Depression in Adults. The Journal of Clinical Psychiatry, 70(7), 983-989. doi:10.4088/JCP.08m04521

Wilde, A., Chan, H.-N., Rahman, B., Meiser, B., Mitchell, P., Schofield, P., \& Green, M. (2014). A meta-analysis of the risk of major affective disorder in relatives of individuals affected 
by major depressive disorder or bipolar disorder. Journal of Affective Disorders, 37-47. doi:10.1016/j.jad.2014.01.014

Wilson, S., Vaidyanathan, U., Miller, M. B., McGue, M., \& lacono, W. G. (2014). Premorbid risk factors for major depressive disorder: are they associated with early onset and recurrent course? Development and Psychopathology, 26(4pt2), 1477-1493. doi:10.1017/S0954579414001151

Wingenfeld, K., Spitzer, C., Mensebach, C., Grabe, H. J., Hill, A., Gast, U.,... Driessen, M. (2010). Die deutsche Version des Childhood Trauma Questionnaire (CTQ): Erste Befunde zu den psychometrischen Kennwerten [The German version of the Childhood Trauma Questionnaire (СTQ): preliminary psychometric properties]. Psychotherapie, Psychosomatik, Medizinische Psychologie, 60(11), 442-450. doi:10.1055/s-0030-1247564

Wittchen, H. U., Jacobi, F., Klose, M., \& Ryl, L. (Eds.) 2010. Themenheft 51 "Depressive Erkrankungen. Gesundheitsberichterstattung-Hefte: Robert Koch-Institut. Retrieved from urn:nbn:de:0257-10010866

Wittchen, H. U., Jacobi, F., Mack, S., Gerschler, A., Scholl, L., Gaebel, W., \& ... \& Siegert, J. (2012). Was sind die häufigsten psychischen Störungen in Deutschland. Robert Koch Institut. DEGS Symposium, 14, 2012.

Wittchen, H. U., Jacobi, F., Rehm, J., Gustavsson, A., Svensson, M., Jonsson, B.,... Steinhausen, H.-C. (2011). The size and burden of mental disorders and other disorders of the brain in Europe 2010. European Neuropsychopharmacology: The Journal of the European College of Neuropsychopharmacology, 21(9), 655-679. doi:10.1016/j.euroneuro.2011.07.018

Wittchen, H.-U., \& Jacobi, F. (2006). Epidemiologie. In G. Stoppe, A. Bramesfeld, \& F.-W. Schwartz (Eds.), Volkskrankheit Depression? Bestandsaufnahme und Perspektiven (pp. 1537). Berlin, Heidelberg: Springer. doi:10.1007/3-540-32221-3_2

Woodberry, K. A., Shapiro, D. I., Bryant, C., \& Seidman, L. J. (2016). Progress and Future Directions in Research on the Psychosis Prodrome: A Review for Clinicians. Harvard Review of Psychiatry, 24(2), 87-103. doi:10.1097/HRP.0000000000000109

Woodward, L. J., \& Fergusson, D. M. (2001). Life course outcomes of young people with anxiety disorders in adolescence. Journal of the American Academy of Child and Adolescent Psychiatry, 40(9), 1086-1093. doi:10.1097/00004583-200109000-00018

Yoon, P. W., Scheuner, M. T., Jorgensen, C., \& Khoury, M. J. (2009). Developing Family Healthware, a family history screening tool to prevent common chronic diseases. Preventing Chronic Disease, 6(1), A33.

Yoon, P. W., Scheuner, M. T., Peterson-Oehlke, K. L., Gwinn, M., Faucett, A., \& Khoury, M. J. (2002). Can family history be used as a tool for public health and preventive medicine? Genetics in Medicine : Official Journal of the American College of Medical Genetics, 4(4), 304-310. 
Yung, A. R., Woods, S. W., Ruhrmann, S., Addington, J., Schultze-Lutter, F., Cornblatt, B. A.,... McGlashan, T. H. (2012). Whither the attenuated psychosis syndrome? Schizophrenia Bulletin, 38(6), 1130-1134. doi:10.1093/schbul/sbs108 


\section{Verwendete Fragbögen}

Berman, A. H., Bergman, H., Palmstierna, T., \& Schlyter, F. (2005). Evaluation of the Drug Use Disorders Identification Test (DUDIT) in criminal justice and detoxification settings and in a Swedish population sample. European Addiction Research, 11(1), 22-31.

Bredle, J. M., Salsman, J. M., Debb, S. M., Arnold, B. J., \& Cella, D. (2011). Spiritual well-being as a component of health-related quality of life: the functional assessment of chronic illness therapy-spiritual well-being scale (FACIT-Sp). Religions, 2(1), 77-94.

Borkenau, P., \& Ostendorf, F. (2008). NEO-Fünf-Faktoren Inventar:(NEO-FFI); nach Costa und McCrae. Göttingen: Hogrefe.

Büssing, A., \& Perrar, K. M. (1992). Die Messung von Burnout. Untersuchung einer deutschen Fassung des Maslach Burnout Inventory (MBI-D). Diagnostica, 38(4), 328-352.

Correll, C. U., Olvet, D. M., Auther, A. M., Hauser, M., Kishimoto, T., Carrión, R. E., ... Cornblatt, B. A. (2014). The Bipolar Prodrome Symptom Interview and ScaleProspective (BPSS-P): description and validation in a psychiatric sample and healthy controls. Bipolar Disorders, 16(5), 505-522.

Erfurt, A., Gerlach, A.L., Hellweg, I. et al. (2005). Studies on a German version of the temperament auto-questionnaire TEMPS-A: construction and validation of the briefTEMPS-M. Journal of Affective Disorders, 85, 53-69.

Forman, T. A., Williams, D. R., \& Jackson, J. S. (1997). Race, Place, and Discrimination. In C. Gardner (Ed.), Perspectives on Social Problems, 9, 231-261.

Garner, D. M., \& Garfinkel, P. E. (1979). The Eating Attitudes Test: An index of the symptoms of anorexia nervosa. Psychological Medicine, 9(2), 273-279.

Gast, U., Rodewald, F., Benecke, H.-H. \& Driessen, M. (2001). Deutsche Bearbeitung des Childhood Trauma Questionnaire (unautorisiert). Unveröffentlihctes Manuskript, Medizinische Hochschule Hannover.

Glaesmer, H., Hoyer, J., Klotsche, J., \& Herzberg, P. Y. (2008). Die deutsche Version des LifeOrientation-Tests (LOT-R) zum dispositionellen Optimismus und Pessimismus. Zeitschrift für Gesundheitspsychologie, 16(1), 26-31.

Hautzinger, M., Bailer, M., Worall, H., \& Keller, F. (1994). Beck Depressions Inventar (BDI). Bearbeitung der deutschen Ausgabe. Testhandbuch. Bern: Huber.

Hautzinger, M., Keller, F., \& Kühner, C. (2006). Beck depressions-inventar (BDI-II). Frankfurt: Harcourt Test Services.

Heatherton, T. F., Kozlowski, L. T., Frecker, R. C., \& Fagerstrom, K. O. (1991). The Fagerström test for nicotine dependence: a revision of the Fagerstrom Tolerance Questionnaire. British Journal of Addiction, 86(9), 1119-1127.

Hjemdal, O., Friborg, O., Braun, S., Kempenaers, C., Linkowski, P., \& Fossion, P. (2011). The Resilience Scale for Adults: Construct validity and measurement in a Belgian sample. International Journal of Testing, 11(1), 53-70. 
Huebner, E. S., Suldo, S., Valois, R. F., Drane, J. W., \& Zullig, K. (2004). Brief Multidimensional Students' life satisfaction scale: sex, race, and grade effects for a high school sample. Psychological Reports, 94(1), 351-356.

Idler, E. L. (1987). Religious involvement and the health of the elderly: Some hypotheses and an initial test. Social Forces, 66(1), 226-238.

Janke, W., Erdmann, G., \& Boucsein, W. (1985). Stressverarbeitungsfragebogen (SVF). Hogrefe: Berlin.

King, M., Walker, C., Levy, G., Bottomley et al. (2008). Development and validation of an international risk prediction algorithm for episodes of major depression in general practice attendees: the PredictD study. Archives of General Psychiatry, 65(12), 13681376.

Klusemann, J., Nikolaides A., Schwickerath, J., Brunn, M., \& Kneip, V. (2008). Trierer Mobbing-Kurz-Skala (TMKS). Validierung eines Screening-Instrumentes zur diagnostischen Erfassung von Mobbing am Arbeitsplatz. Praxis Klinische Verhaltensmedizin und Rehabilitation, 82, 323-334.

Krampen, G. (1991). FKK Fragebogen zu Kompetenz-und Kontrollüberzeugungen. Göttingen: Hogrefe.

Kühner, C., Huffziger, S., \& Nolen-Hoeksema, S. (2007). Response styles questionnaire: RSQD. Berlin: Hogrefe

Löwe, B. P. (Ed.). (2001). Gesundheitsfragebogen für Patienten PHQ-D: Manual; Komplettversion und Kurzform. Karlsruhe: Pfizer

Maier-Diewald, W. (1983). Die Münchner Ereignisliste (MEL): Anwendungsmanual. MaxPlank-Institut für Psychiatrie.

Paxton, A. E., Strycker, L. A., Toobert, D. J., Ammerman, A. S., \& Glasgow, R. E. (2011). Starting the conversation: performance of a brief dietary assessment and intervention tool for health professionals. American Journal of Preventive Medicine, 40(1), 67-71.

Poppe, P., Pelster, A., \& Stiensmeier-Pelster, J. (2005). ASF-E: Attributionsstil-Fragebogen für Erwachsene. Berlin: Hogrefe.

Pullmann, H., \& Allik, J. (2000). The Rosenberg Self-Esteem Scale: its dimensionality, stability and personality correlates in Estonian. Personality and Individual Differences, 28(4), 701-715.

Saunders, J. B., Aasland, O. G., Babor, T. F., De la Fuente, J. R., \& Grant, M. (1993). Development of the alcohol use disorders identification test (AUDIT). WHO collaborative project on early detection of persons with harmful alcohol consumption-II. Addiction, 88(6), 791-804.

Schultze-Lutter, F., \& Koch, E. (2010). Schizophrenia Proneness Instrument: child and youth version (SPI-CY). Rome: Fioriti.

Schulz, P., Becker, P., \& Schlotz, W. (2004). TICS: Trierer Inventar zum chronischen Stress: Manual. Berlin: Hogrefe. 
Sheldon, K. M. (1995). Creativity and self-determination in personality. Creativity Research Journal, 8(1), 25-36.

Stöber, J. (2003). „Frost Multidimensional Perfectionism Scale “(FMPS). 10 Fragebögen und Ratingskalen zur Zwangsstörung. In J. Hoyer \& J. Margraf (Eds.), Angstdiagnostik (S. 321-370). Berlin Heidelberg: Springer.

Traue, H. C., Hrabal, V., \& Kosarz, P. (2000). Alltagsbelastungsfragebogen (ABF): Zur inneren Konsistenz, Validierung und Stressdiagnostik mit dem deutschsprachigen daily stress inventory. Verhaltenstherapie und Verhaltensmedizin, 21(1), 15-38.

WHOQOL Group. (1998). Development of the World Health Organization WHOQOL-BREF quality of life assessment. Psychological Medicine, 28(03), 551-558.

Williams, D.R., YU, Y., Jackson, J.S., \& Anderson, N.B. (1997). Racial Differences in Physical and Mental Health: Socioeconomic Status, Stress, and Discrimination. Journal of Health Psychology, 2 (3), 335-351.

Wilson, M. M. G., Thomas, D. R., Rubenstein, L. Z., Chibnall, J. T., Anderson, S., Baxi, A., ... \& Morley, J. E. (2005). Appetite assessment: simple appetite questionnaire predicts weight loss in community-dwelling adults and nursing home residents. The American Journal of Clinical Nutrition, 82(5), 1074-1081.

Wittchen, H. U., Wunderlich, U., Gruschwitz, S., \& Zaudig, M. (1997). SKID-I. Strukturiertes klinisches Interview für DSM-IV. Göttingen: Hogrefe.

Zimet, G. D., Dahlem, N. W., Zimet, S. G., \& Farley, G. K. (1988). The multidimensional scale of perceived social support. Journal of Personality Assessment, 52(1), 30-41. 
Anhang 


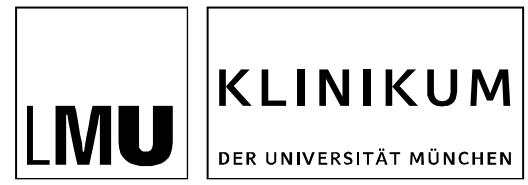

CAMPUS INNENSTADT UND GROSSHADERN

INTERDISZIPLINÄRES ZENTRUM FÜR PRÄVENTION PSYCHISCHER ERKRANKUNGEN DER UNIVERSITÄT MÜNCHEN - CEP ${ }^{L M}$

\section{Phase 1 DEEP-Studie Kodierung}

Name des Interviewten

Name, Vorname [Mutter] (Geburtsname)
Geburtsjahr des Interviewten

Name, Vorname [Vater] (Geburtsname)

\section{Probandenkodierung}

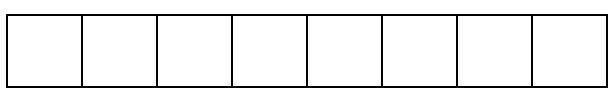

Die Kodierung setzt sich zusammen aus den Initialen der Mutter und den Initialen des Vaters des Studienteilnehmers und einem vierstelligen Zufallscode.

Beispiel:

Geburtsname der Mutter: Marie Becker Geburtsname des Vaters: Hans Müller Zufallscode: 9999 


\section{Phase 1 DEEP-Interview}

\section{Probandenkodierung}

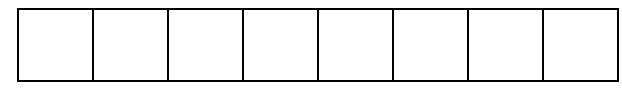

\section{Zuweisung:}

$\square$ LMU Psychiatrie stationär

$\square$ Extern, Sonstige/Aushänge: 


\section{Soziodemographische Angaben}

\section{Allgemeines}

Geschlecht:

Geburtsdatum

$x x . y y . z z z z$

Gewicht

BMI

Raucher

Jemals geraucht?

Händigkeit:

Geburtsort

Nationalität:

Zwei-/Mehrsprachig:

O männlich

weiblich

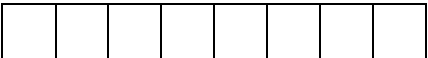

Alter in Jahren:

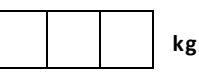

Größe

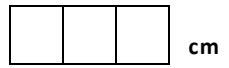

$\rightarrow$ wird vom Untersuchungsleiter ausgefüllt!

O nein O ja wieviel?

Zigaretten/Tag $\rightarrow$ seit wann

(Monate)

$\mathrm{O}$ nein $\mathrm{O}$ ja wieviel?

Zigaretten/Tag $\rightarrow$ wie lange? (Monate)

O rechtshändig

O linkshändig

$\mathrm{O}$ beidhändig

\section{Geburtsland:}

Muttersprache:

$\mathrm{O} \mathrm{ja}$

O nein

\section{Familie und Wohnsituation}

Familienstand:

Kinder

Kinder wie viele:
$O$ verheiratet

$\mathrm{O}$ alleinstehend

$\mathrm{O}$ verwitwet, seit

O nein

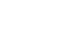

in fester Partnerschaft, nicht verheiratet

$O$ geschieden / getrennt, seit

$\mathrm{O}$ ja

\begin{tabular}{|r|r|}
\hline Kind & Geburtsjahr \\
\hline 1 & \\
\hline 2 & \\
\hline 3 & \\
\hline 4 & \\
\hline 5 & \\
\end{tabular}

mit Familie (Ehepartner, Eltern, Kinder)

O Wohngemeinschaft

O unbekannt/unklar
O betreutes Wohnen

O ohne festen Wohnsitz 


\section{Ausbildung und Beruf}

\begin{tabular}{|c|c|c|c|}
\hline Schuljahre: & O 6. Klasse & O 8. Klasse & O 9. Klasse \\
\hline & O 10. Klasse & O 12./13. Klasse & O sonstiges \\
\hline Höchster Schulabschluss: & O keinen & O Hauptschule & O Realschule \\
\hline & O Abitur & O Ausbildung & O Fachhochschule \\
\hline & O Studium & O Promotion/Habilit & \\
\hline Berufstätigkeit aktuell: & O nein & $\mathrm{O}$ ja & $\mathrm{O}$ arbeitssuchend \\
\hline & O Schüler/in & O Student/in & $O$ in Ausbildung (AZUBI) \\
\hline & O Rentner/in & O Hausfrau/-mann & \\
\hline Tätigkeit/ & & & \\
\hline Studienfach aktuell: & -_-_-_-_-_-_-_-_-_-_- & _-_seit:__-__-_ & \\
\hline Beruflicher Werdegang: & von:_________bis:_______ & __-_Tätigkeit._-___-__ & \\
\hline & von:__________bis:______ & ___Tätigkeit__. & \\
\hline & von:__________bis:______ & ___Tätigkeit___ & \\
\hline & von:________bis:_______ & ___Tätigkeit_. & \\
\hline & von:__________bis:_______ & ___Tätigkeit___ & \\
\hline & von:__________bis:_______ & ___ Tätigkeit___ & --_-_-_-_--_--_--_- \\
\hline Berentung/Pensionierung: & seit: _-_-_-_-_-_- & -- & \\
\hline Berufsgruppe aktuell: & O Angestellte(r) & O Beamte(r) & O leitende(r) Angestellte(r) \\
\hline & O Freiberufler/Selbständig & O Sonstige & \\
\hline Nacht-/Schichtarbeit je: & O nein & O ja, aktuell & \\
\hline & O ja, früher:_-__-_-_-_-_ & & \\
\hline monatliches Einkommen: & O unter 450 Euro & O $451-850$ Euro & O $851-1500$ Euro \\
\hline (Netto) & O $1501-2500$ Euro & O 2501 - 3500 Euro & O über 3500 Euro \\
\hline
\end{tabular}




\section{Screening - Aktuelle somatische Erkrankungen/Medikamente}

Schweregrad

1 kein Problem

2 leichtes Problem, beeinflusst die Alltagsaktivitäten nicht; Behandlung erforderlich oder auch nicht; prognostisch nicht relevant (z. B. Hauterkrankungen, Hernien, Hämorrhoiden)

3 mäßiges Problem, alltagsrelevant; Behandlung ist notwendig; Prognose ist gut (z. B. Gallensteine, Diabetes, Frakturen)

4 schweres Problem, Behinderung oder Handicap; Behandlung dringend erforderlich; Prognose zweifelhaft (z. B. operables Ca., Emphysem, Herzinsuffizienz)

5 lebensbedrohlich; Behandlung dringlich oder aussichtslos; Prognose kritisch (z. B. Herzinfarkt, Schlaganfall, gastrointestinale Blutung, Embolie)

1. Leiden Sie derzeit an...

\begin{tabular}{|c|c|c|c|c|c|}
\hline \multirow{2}{*}{$\begin{array}{l}\text { Diagnose } \\
\text { Herzerkrankung oder Herz-Kreislauferkrankungen? } \\
\text { (z.B. Herzinfarkt, koronare Herzerkrankung, Herzklappenfehler, Rhythmusstörungen, } \\
\text { Herzinsuffizienz, Schlaganfall) }\end{array}$} & \multicolumn{5}{|c|}{ Schweregrad } \\
\hline & 1 & 2 & 3 & 4 & 5 \\
\hline Bluthochdruck? & 1 & 2 & 3 & 4 & 5 \\
\hline $\begin{array}{l}\text { Bluterkrankung oder Blutgefäßerkrankung? } \\
\text { (z. B. Anämie, Leukämie, lymphatische Erkrankung, AVK, Thrombose) }\end{array}$ & 1 & 2 & 3 & 4 & 5 \\
\hline $\begin{array}{l}\text { Atemwegserkrankung? } \\
\text { (z. B. Chronische Bronchitis, Asthma, Emphysem, Tb) }\end{array}$ & 1 & 2 & 3 & 4 & 5 \\
\hline $\begin{array}{l}\text { Erkrankung der Augen, Ohren, Nase, Kehlkopf? } \\
\text { (z. B. Cataract, Glaukom, Hypakusis) }\end{array}$ & 1 & 2 & 3 & 4 & 5 \\
\hline $\begin{array}{l}\text { Magen, Zwölffingerdarm, Galle, Bauchspeicheldrüse? } \\
\text { (z. B. Gastritis, Geschwür, Gallensteine, Pankreatitis) }\end{array}$ & 1 & 2 & 3 & 4 & 5 \\
\hline Darmerkrankung? (z. B. Polyp, Colitis) & 1 & 2 & 3 & 4 & 5 \\
\hline Lebererkrankung? (z. B. Fettleber, Hepatitis, Leberzirrhose) & 1 & 2 & 3 & 4 & 5 \\
\hline $\begin{array}{l}\text { Nierenerkrankung? } \\
\text { (z. B. Nierensteine, Schrumpfniere, Niereninsuffizienz) }\end{array}$ & 1 & 2 & 3 & 4 & 5 \\
\hline $\begin{array}{l}\text { Urologische oder gynäkologische Erkrankung? } \\
\text { (z. B. Prostata, Blase, Brust, Gebärmutter) }\end{array}$ & 1 & 2 & 3 & 4 & 5 \\
\hline $\begin{array}{l}\text { Muskel- und Skelettsystem, Haut? } \\
\text { (z. B. Arthrose, Osteoporose, Rheuma) }\end{array}$ & 1 & 2 & 3 & 4 & 5 \\
\hline $\begin{array}{l}\text { Neurologische Erkrankung? } \\
\text { (z. B. Parkinson, MS, Epilepsie, Rückenmark) }\end{array}$ & 1 & 2 & 3 & 4 & 5 \\
\hline $\begin{array}{l}\text { Endokrinologische oder Stoffwechselerkrankung? } \\
\text { (z. B. Schilddrüse, Diabetes) }\end{array}$ & 1 & 2 & 3 & 4 & 5 \\
\hline $\begin{array}{l}\text { Psychiatrische Erkrankung? } \\
\text { (z. B. Demenz, Depression, Angst, Schizophrenie) }\end{array}$ & 1 & 2 & 3 & 4 & 5 \\
\hline 2. Haben Sie eine - noch nicht genannte - Erkrankung bzw. körperliche Beschwerden? (Allergien & & & & & \\
\hline$\square$ ja, welche? & & & & & \\
\hline 3. Waren Sie in den letzten 12 Monaten aus Krankheitsgründen beim Arzt? & & & & & \\
\hline$\square \mathrm{ja}, \quad$ wie oft? __ mal & & & & & \\
\hline
\end{tabular}


4. Haben Sie eine chronische Erkrankung?

$\square$ nein $\quad \square$ ja, welche? seit wann

5. Nehmen Sie derzeit Medikamente ein?

$\square$ nein

$\square$ ja, welche?

\begin{tabular}{|l|l|l|l|}
\hline \multicolumn{1}{|c|}{ Medikament } & Dosierung & Einnahmeschema & seit \\
\hline 1. & & & \\
\hline 2. & & & \\
\hline 3. & & & \\
\hline 4. & & & \\
\hline 5. & & & \\
\hline
\end{tabular}

6. Nahmen Sie jemals Psychopharmaka ein?

$\square$ nein

$\square$ ja, welche?

\begin{tabular}{|l|l|l|l|}
\hline \multicolumn{1}{|c|}{ Medikament } & Dosierung & wann? (Zeitraum) & wie lange?(Monate) \\
\hline 1. & & & \\
\hline 2. & & & \\
\hline 3. & & & \\
\hline 4. & & & \\
\hline 5. & & & \\
\hline
\end{tabular}

7. Konsumieren Sie Drogen/Rauschmittel?

$\square$ nein

$\square$ ja, welche?

\begin{tabular}{|l|l|l|l|}
\hline \multicolumn{1}{|c|}{ Substanz } & Häufigkeit/Monat & Dosis & seit / Zeitraum \\
\hline 1. & & & \\
\hline 2. & & & \\
\hline 3. & & & \\
\hline
\end{tabular}

8. Nahmen Sie in der Zeit VOR Ihrer erstmalig auftretenden depressiven Erkrankung (andere) Medikamente/Rauschmittel ein?
$\square$ nein
$\square$ ja, welche?

\begin{tabular}{|l|l|l|l|}
\hline \multicolumn{1}{|c|}{ Medikament } & Dosierung & Einnahmeschema & Zeitraum \\
\hline 1. & & & \\
\hline 2. & & & \\
\hline 3. & & & \\
\hline
\end{tabular}

9. Schädeltrauma + Bewusstlosigkeit $>5 \mathrm{~min}$ ?

$\square$ nein $\square$ ja, wann?

10. Somatische/neurologische Erkrankungen mit Auswirkungen auf Gehirn?

$\square$ nein $\square$ ja, welche? 


\section{DEEP-Explorationsleitfaden}

1. Haben Sie schon einmal wegen psychischer Probleme Hilfe aufgesucht (ambulant)?

$\square$ nein

$\square$ ja

\begin{tabular}{|l|l|l|}
\hline Zeitraum & $\begin{array}{l}\text { Art (Beratungsstelle, Psychiater, } \\
\text { Psychologe, Psychotherapeut...) }\end{array}$ & Behandlungsgrund/Ursache \\
\hline 1. & & \\
\hline 2. & & \\
\hline 3. & & \\
\hline 4. & & \\
\hline
\end{tabular}

2. Waren Sie schon einmal wegen psychischer Probleme in stationärer Behandlung?

$\square$ nein

$\square$ ja

\begin{tabular}{|l|lr|l|}
\hline Zeitraum (Monat/Jahr - Monat/Jahr) & Art der Klinik & Behandlungsgrund/Ursache \\
\hline 1. & $\square$ Psychiatrie & $\square$ Psychosomatik & \\
\hline 2. & $\square$ Psychiatrie & $\square$ Psychosomatik & \\
\hline 3. & $\square$ Psychiatrie & $\square$ Psychosomatik & \\
\hline 4. & $\square$ Psychiatrie & $\square$ Psychosomatik & \\
\hline
\end{tabular}

3. Waren Sie jemals wegen der Behandlung eines körperlichen Problems in einem Krankenhaus?

$\square$ nein

$\square$ ja

\begin{tabular}{|l|l|l|}
\hline Zeitraum & Art der Klinik & Behandlungsgrund/Ursache \\
\hline 1. & & \\
\hline 2. & & \\
\hline 3. & & \\
\hline 4. & & \\
\hline
\end{tabular}

4. Wurden Sie schon einmal wegen Medikamenten-, Alkohol- oder Drogenprobleme behandelt?
$\square$ nein
$\square$ ja

5. Haben Sie jemals versucht sich selbst zu verletzen oder umzubringen?

$\square$ nein

$\square$ ja

wann? /wie

6. Gibt es in Ihrer Familie psychische Erkrankung?

$\square$ nein

Wenn ja, wer ist/war erkrankt?
$\square$ Vater
$\square$ Mutter
$\square$ Geschwister:
$\square$ Großeltern:

$\square$ Kinder: $\square$ andere

wenn ja, welche?

$\begin{array}{llll}\square \text { Depression } & \square \text { Angsterkrankungen, Phobien } & \square \text { bipolare Erkrankung } & \square \text { Zwangserkrankung } \\ \square \text { Schizophrenie } & \square \text { ADHS } & \square \text { Sucht } \quad \square \text { andere }\end{array}$

Erkranktes Familienmitglied jemals in Behandlung?
$\square$ nein
$\square$ ambulant
$\square$ stationär 
7. Haben Sie Angehörige in Ihrer Familie, die einen Suizid bzw. Suizidversuch begingen?

$\square$ nein

$\square \mathrm{ja}$

Wer, wann?

\section{Screening Psychiatrische Erkrankungen (SKID)}

\begin{tabular}{|c|c|c|}
\hline & Frage & Hinweis auf \\
\hline 1 & $\begin{array}{l}\text { Hatten Sie jemals Angst, allein das Haus zu verlassen, sich in einer Menschenmenge } \\
\text { zu befinden, in einer Schlange anzustehen oder mit dem Zug oder Bus zu fahren? } \\
\text { fhein fja }\end{array}$ & $\begin{array}{l}\text { Panikstörung/ } \\
\text { Agoraphobie } \\
\text { (F1-F40) }\end{array}$ \\
\hline 2 & $\begin{array}{l}\text { Hatten Sie jemals in Ihrem Leben einen Angstanfall, bei dem Sie ganz plötzlich in } \\
\text { panischen Schrecken gerieten oder starke Angst hatten? } \\
\text { fnein } \quad \mp \text { ja }\end{array}$ & $\begin{array}{l}\text { Panikstörung/ } \\
\text { Agoraphobie } \\
\text { (F1-F40) }\end{array}$ \\
\hline 3 & $\begin{array}{l}\text { Hatten Sie schon einmal Angst davor, oder war es Ihnen unangenehm, in Gegenwart } \\
\text { anderer Menschen zu sprechen, zu essen oder zu schreiben? } \\
\text { fnein } \quad \text { ¡ja }\end{array}$ & $\begin{array}{l}\text { Soziale Phobie (F41- } \\
\text { F55) }\end{array}$ \\
\hline 4 & $\begin{array}{l}\text { Gibt es noch andere Dinge, vor denen Sie besonders Angst haben, wie z.B. in einem } \\
\text { Flugzeug zu sitzen, Blut zu sehen, sich in geschlossenen Räumen aufzuhalten, vor } \\
\text { bestimmten Tieren oder vor Höhen? } \\
\text { fhein } \quad \text { †ja }\end{array}$ & $\begin{array}{l}\text { Spezifische Phobie } \\
\text { (F56-F67) }\end{array}$ \\
\hline 5 & $\begin{array}{l}\text { Waren Sie in den letzten } 6 \text { Monaten besonders nervös oder ängstlich? } \\
\text { fnein } \quad \mp \text { ja }\end{array}$ & $\begin{array}{l}\text { Generalisierte } \\
\text { Angststörung (F115- } \\
\text { F132) }\end{array}$ \\
\hline 6 & $\begin{array}{l}\text { Haben Sie jemals unter Gedanken gelitten, die unsinnig waren und immer wieder } \\
\text { kamen, auch wenn Sie es gar nicht wollten? } \\
\text { Thein } \quad \text { ¡ja }\end{array}$ & $\begin{array}{l}\text { Zwangsstörung: } \\
\text { Zwangsgedanken } \\
\text { (F68-F72; F75-F84) }\end{array}$ \\
\hline 7 & $\begin{array}{l}\text { Ist es schon einmal vorgekommen, dass Sie bestimmte Dinge immer und immer } \\
\text { wieder tun mussten, z.B. sich die Hände zu waschen/etwas mehrmals zu } \\
\text { kontrollieren, um sicherzugehen, dass Sie es richtig gemacht haben? } \\
\text { Thein } \quad \text { jja }\end{array}$ & $\begin{array}{l}\text { Zwangsstörung: } \\
\text { Zwangshandlungen } \\
\text { (F75-F84) }\end{array}$ \\
\hline 8 & $\begin{array}{l}\text { Waren Sie schon einmal wegen körperlicher Beschwerden mehrfach bei einem oder } \\
\text { mehreren Ärzten ohne dass diese die Beschwerden hinreichend erfolgreich } \\
\text { behandeln oder erklären konnten? } \\
\text { Thein } \quad \text { ¡ja }\end{array}$ & $\begin{array}{l}\text { Somatoforme } \\
\text { Störungen } \\
\text { (G1-G52) }\end{array}$ \\
\hline 9 & $\begin{array}{l}\text { Machen Sie sich unablässig Sorgen darüber, eine ernsthafte Erkrankung zu haben? } \\
\text { Thein †ja }\end{array}$ & $\begin{array}{l}\text { Hypochondrische } \\
\text { Störung } \\
\text { (G53-G63) }\end{array}$ \\
\hline 10 & $\begin{array}{l}\text { Haben Sie schon mal ein besonders belastendes Ereignis erlebt und danach unter } \\
\text { häufig wiederkehrenden und sich aufdrängenden Erinnerungen gelitten? } \\
\text { ¡nein } \quad \text { †ja }\end{array}$ & $\begin{array}{l}\text { PTSD } \\
\text { (F85-F114) } \\
\text { Akute Stress-störung } \\
\text { (J1-J11) }\end{array}$ \\
\hline 11 & $\begin{array}{l}\text { Vermeiden Sie Situationen, die mit diesem Ereignis zu tun haben oder Sie daran } \\
\text { erinnern? } \\
\text { fhein †ja }\end{array}$ & $\begin{array}{l}\text { PTSD } \\
\text { (F85-F114) } \\
\text { Akute Stress-störung } \\
\text { (J1-J11) }\end{array}$ \\
\hline 12 & $\begin{array}{l}\text { Nach einem solchen besonders belastenden Ereignis: Waren Sie besonders } \\
\text { niedergeschlagen, ängstlich und/oder besonders auffällig oder andersartig in Ihrem } \\
\text { Verhalten? }\end{array}$ & $\begin{array}{l}\text { Anpassungsstörung } \\
\text { (11-17) }\end{array}$ \\
\hline
\end{tabular}




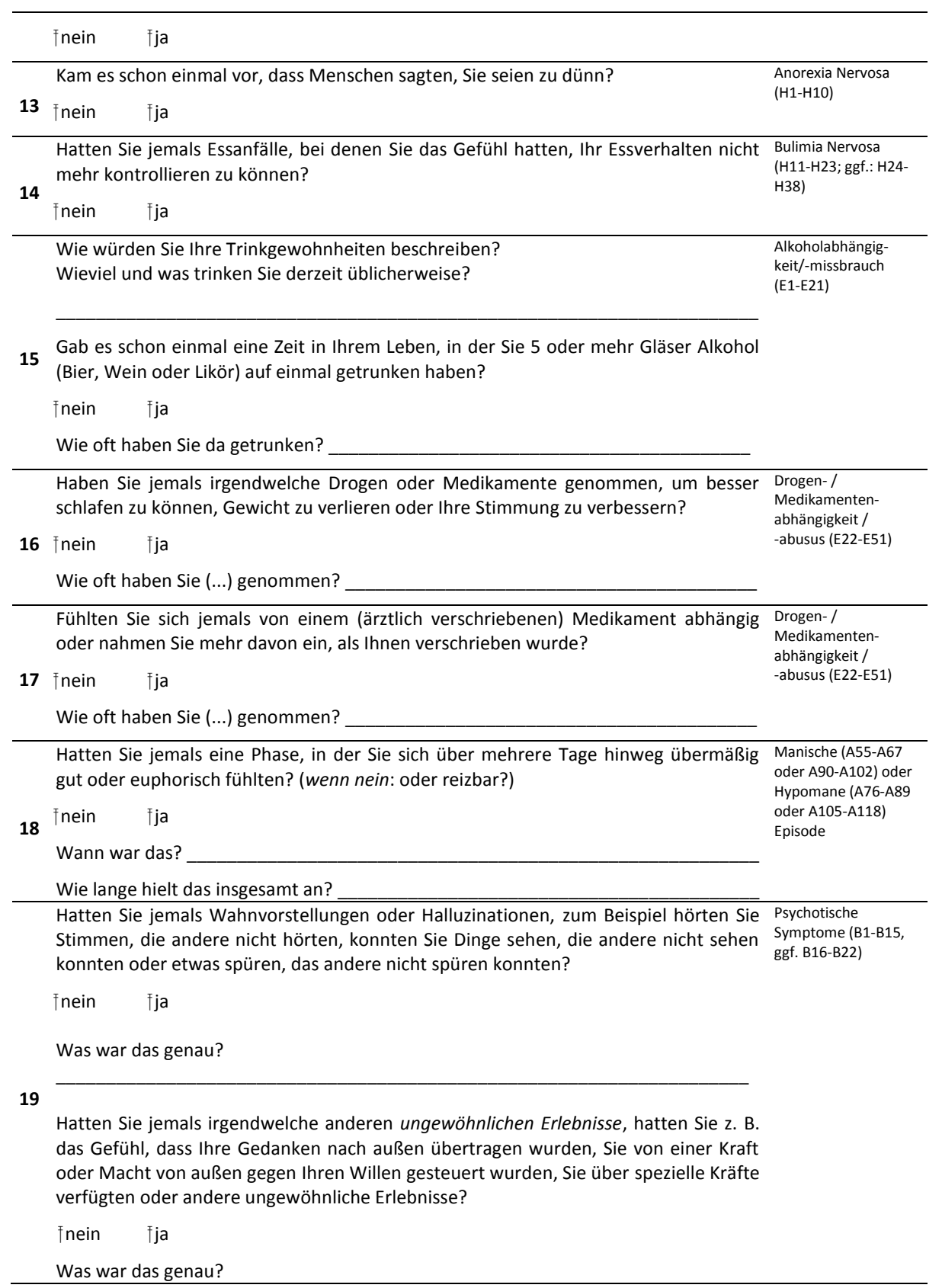




\section{DEEP: Phasen und Intervalle depressiver Erkrankungen}

I. Beginn und Verlauf der letzten Krankheitsphase

1 Gegenwärtige Diagnosen nach ICD-10

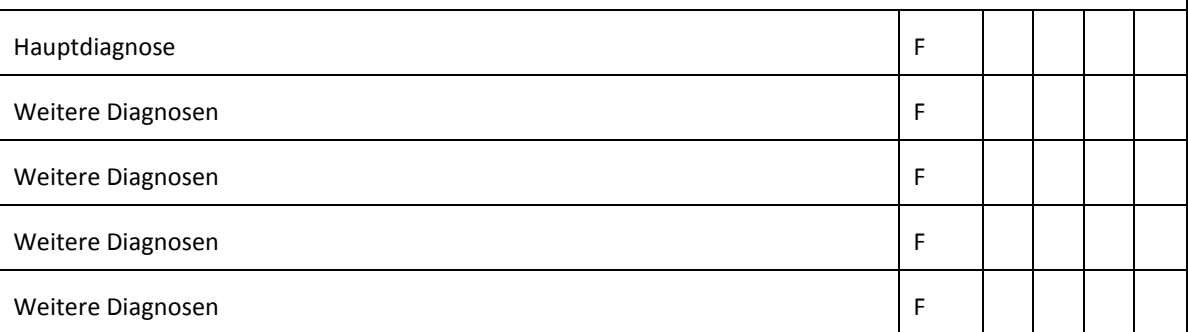

2 Beginn des letzten Aufenthaltes/der Behandlung

$3 \quad$ Beginn der letzten Krankheitsphase

$4 \quad$ Ggf. Ende der letzten Krankheitsphase

5 Hauptsymptome während der letzten Krankheitsphase

a)

b)

c)

d)

Erstkontakt mit einer medizinischen/psychiatrischen Einrichtung während der letzten Krankheitsphase

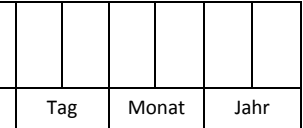

\begin{tabular}{|c|l|}
\hline 7 & Art der Einrichtung bei Erstkontakt \\
\hline$\square$ & Hausarzt \\
\hline$\square$ & Psychiater/Neurologe \\
\hline$\square$ & Psychotherapeut/Psychologe \\
\hline$\square$ & Andere: \\
\hline
\end{tabular}




\begin{tabular}{|c|c|c|c|}
\hline 8 & Diagnosestellung bei letzter Krankheitsphase durch: & & \\
\hline$\square$ & Hausarzt & & \\
\hline & & Monat & Jahr \\
\hline$\square$ & Psychiater/Neurologe & & \\
\hline & & Monat & Jahr \\
\hline$\square$ & Psychotherapeut/Psychologe & & \\
\hline & & Monat & Jahr \\
\hline$\square$ & Klinik/stationärer Aufenthalt & & \\
\hline & & Monat & Jahr \\
\hline$\square$ & Sonstige: & & \\
\hline & & Monat & Jahr \\
\hline
\end{tabular}

$9 \quad$ Auslöser der letzten Krankheitsphase

\begin{tabular}{|l|l|}
\hline & a) \\
\hline & b) \\
\hline & c) \\
\hline & d) \\
\hline & e) \\
\hline
\end{tabular}

\section{Psychische Frühwarnsymptome/}

Früheste Anzeichen der letzten Krankheitsphase, meist schleichender Beginn, weniger stark ausgeprägt als Symptome, z.B. Reizbarkeit, Schlafschwierigkeiten etc.

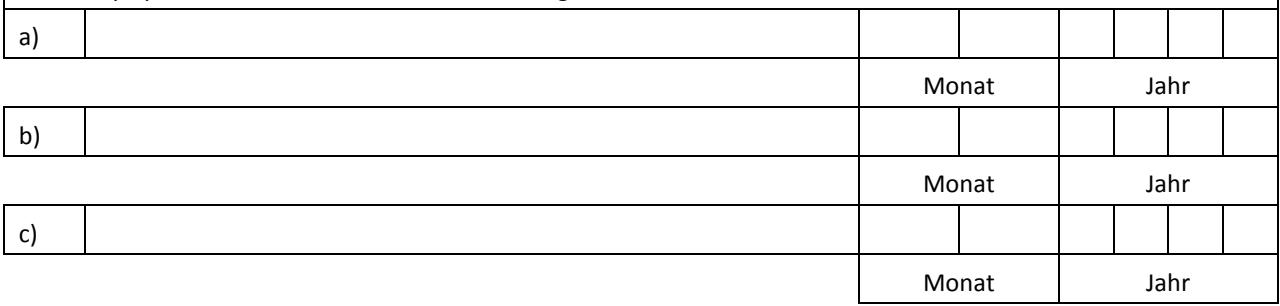

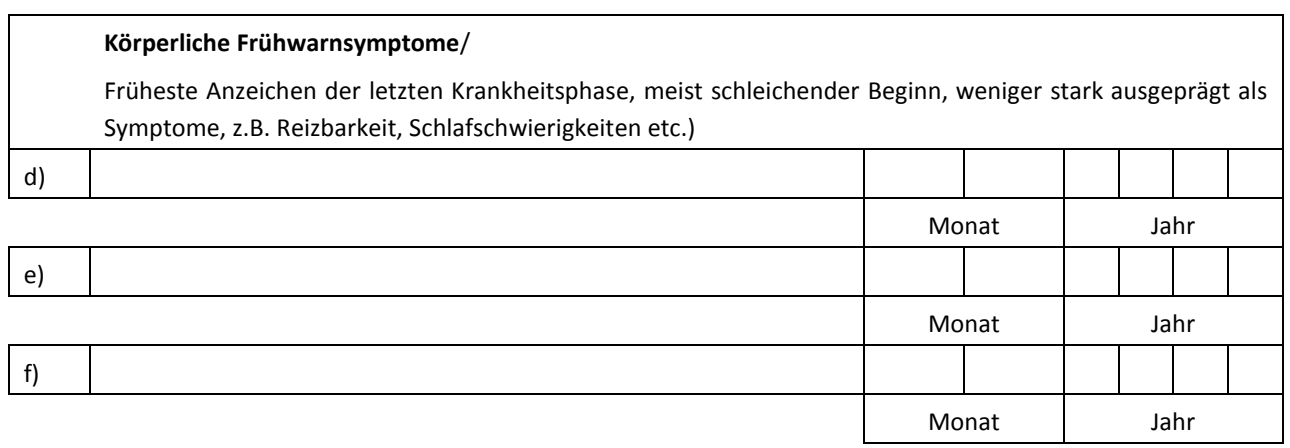




\section{Anzahl und Dauer früherer Krankheitsphasen}

11 Anzahl früherer Krankheitsphasen

\begin{tabular}{|l|l|l|l|l|l|l|l|l|l|l|l|}
\hline 12 & \multicolumn{5}{|c|}{ Beginn } & \multicolumn{5}{|c|}{ Ende } \\
\hline & Von Fachpersonal diagnostiziert? $\square$ nein $\square$ ja & & & & & & & & & \\
\hline
\end{tabular}

\begin{tabular}{|l|l|l|l|l|l|l|l|l|l|l|l|l|}
\hline \multirow{2}{*}{ Weitere Krankheitsphase } & \multicolumn{5}{|c|}{ Beginn } & \multicolumn{5}{c|}{ Ende } \\
\hline & von Fachpersonal diagnostiziert? $\square$ nein $\square$ ja & & & & & & & & & & \\
\hline
\end{tabular}

\begin{tabular}{|c|c|c|c|c|c|}
\hline 14 & Weitere Krankheitsphase & \multicolumn{2}{|c|}{ Beginn } & \multicolumn{2}{|c|}{ Ende } \\
\hline & von Fachpersonal diagnostiziert? $\square$ nein $\square$ ja & & & & \\
\hline & & Monat & Jahr & Monat & Jahr \\
\hline 15 & Weitere Krankheitsphase & \multicolumn{2}{|c|}{ Beginn } & \multicolumn{2}{|c|}{ Ende } \\
\hline & von Fachpersonal diagnostiziert? $\square$ nein $\square$ ja & & & & \\
\hline & & Monat & Jahr & Monat & Jahr \\
\hline 16 & Weitere Krankheitsphase & \multicolumn{2}{|c|}{ Beginn } & \multicolumn{2}{|c|}{ Ende } \\
\hline & von Fachpersonal diagnostiziert? $\square$ nein $\square$ ja & & & & \\
\hline & & Monat & Jahr & Monat & Jahr \\
\hline 17 & Weitere Krankheitsphase & \multicolumn{2}{|c|}{ Beginn } & \multicolumn{2}{|c|}{ Ende } \\
\hline & von Fachpersonal diagnostiziert? $\square$ nein $\square$ ja & & & & \\
\hline & & Monat & Jahr & Monat & Jahr \\
\hline 18 & Weitere Krankheitsphase & \multicolumn{2}{|c|}{ Beginn } & \multicolumn{2}{|c|}{ Ende } \\
\hline & von Fachpersonal diagnostiziert? $\square$ nein $\square$ ja & & & & \\
\hline & & Monat & Jahr & Monat & Jahr \\
\hline
\end{tabular}

19 Krankheitsverlauf

$\square$ Erstmanifestation

$\square$ Rezidivierender Verlauf 
Lebenslinie (hier den Verlauf der Erkrankungsphasen einzeichnen; diagnostizierte Erkrankung markieren, davon Indexphase kennzeichnen Übermäßig gehobene Stimmung, vermehrter Antrieb

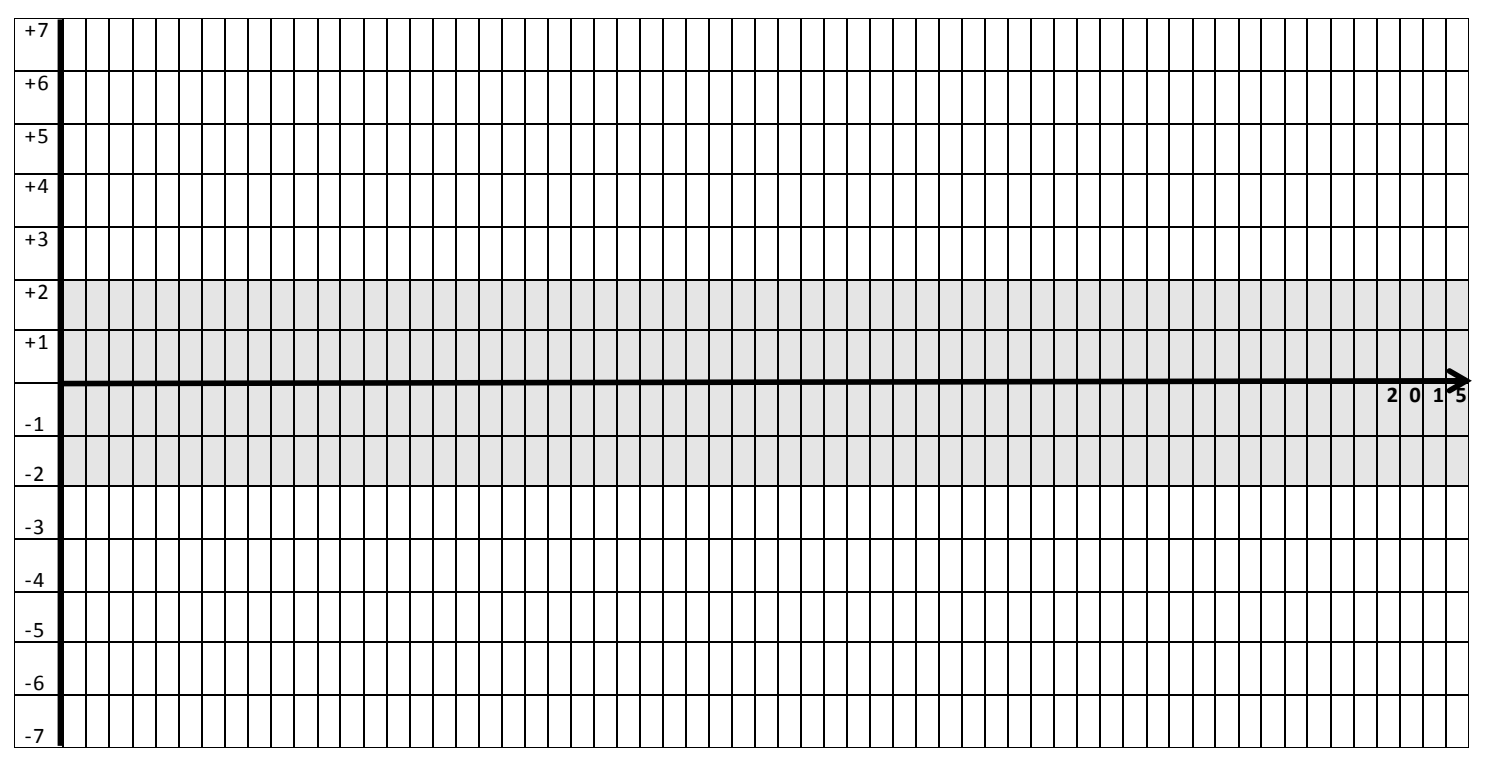

Gedrückte Stimmung, verminderter Antrieb, Freud- und Interessenlosigkeit 
Verlauf der Indexphase

(nur auszufüllen, wenn Indexphase nicht die letzte Phase ist)

\begin{tabular}{|c|c|c|}
\hline 20 & Indexphase = letzte Phase & Indexphase = Wievielte Krankheitsphase? \\
\hline \multirow[t]{6}{*}{21} & Hauptsymptome während der Indexphase & \\
\hline & a) & \\
\hline & b) & \\
\hline & c) & \\
\hline & d) & \\
\hline & e) & \\
\hline
\end{tabular}

22 Erstkontakt mit einer medizinischen/psychiatrischen Einrichtung während der Indexphase

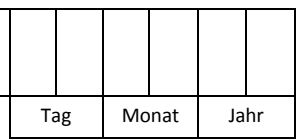

\begin{tabular}{|c|l|}
\hline 23 & Art der Einrichtung bei Erstkontakt \\
\hline$\square$ & Hausarzt \\
\hline$\square$ & Psychiater/Neurologe \\
\hline$\square$ & Psychotherapeut/Psychologe \\
\hline$\square$ & Andere: \\
\hline
\end{tabular}

\begin{tabular}{|c|c|c|c|}
\hline 24 & Diagnosestellung bei Indexphase durch: & & \\
\hline$\square$ & Hausarzt & & \\
\hline & & Monat & Jahr \\
\hline$\square$ & Psychiater/Neurologe & & \\
\hline & & Monat & Jahr \\
\hline$\square$ & Psychotherapeut/Psychologe & & \\
\hline & & Monat & Jahr \\
\hline$\square$ & Klinik/stationärer Aufenthalt & & \\
\hline & & Monat & Jahr \\
\hline$\square$ & Sonstige: & & \\
\hline & & Monat & Jahr \\
\hline
\end{tabular}

\begin{tabular}{|l|l|}
\hline 25 & Auslöser der Indexphase \\
\hline & a) \\
\hline & b) \\
\hline & c) \\
\hline & d) \\
\hline & e) \\
\hline
\end{tabular}




\section{Psychische Frühwarnsymptome der Indexphase/}

Früheste Anzeichen der letzten Krankheitsphase, meist schleichender Beginn, weniger stark ausgeprägt als Symptome, z.B. Reizbarkeit, Schlafschwierigkeiten etc.

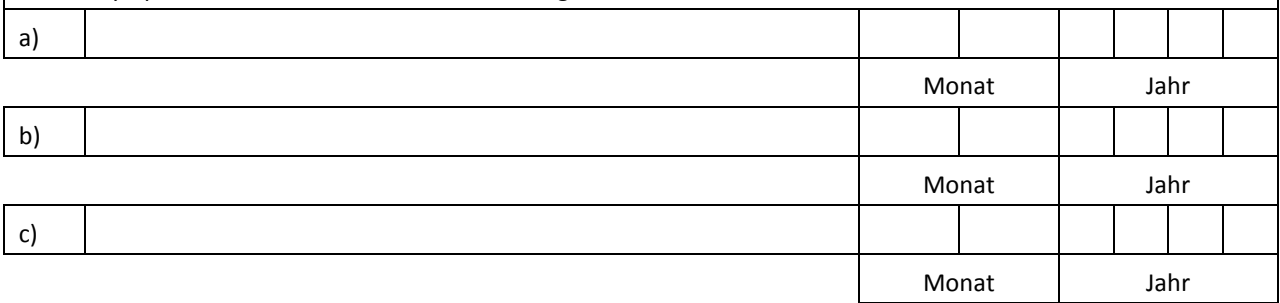

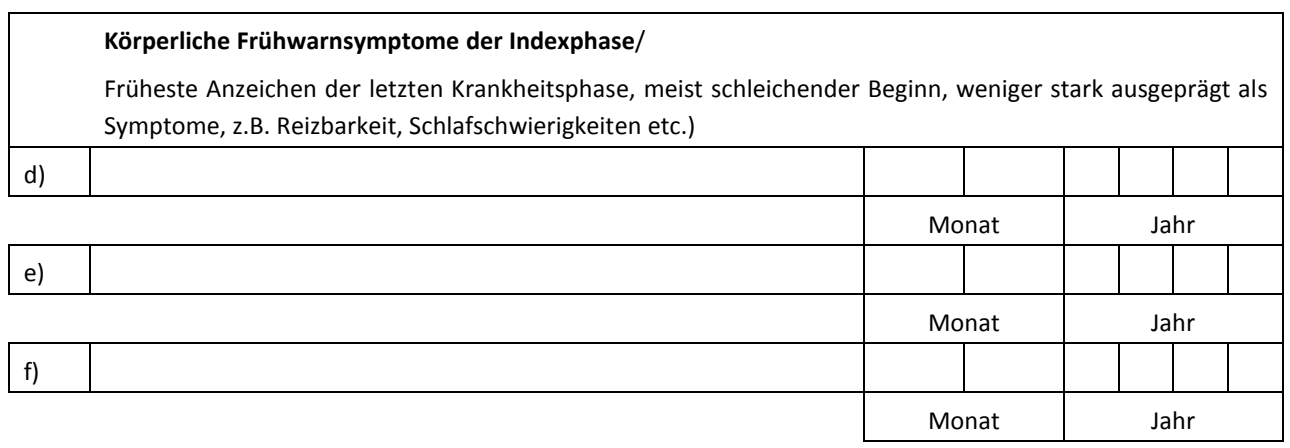




\section{Eidesstattliche Versicherung}

\section{Stegmüller, Veronika}

Ich erkläre hiermit an Eides statt, dass ich die vorliegende Dissertation mit dem Thema

Frühererkennung der Depression. Spezifische Risikofaktoren und Charakteristika der Prodromalphase.

selbständig verfasst, mich außer der angegebenen keiner weiteren Hilfsmittel bedient und alle Erkenntnisse, die aus dem Schrifttum ganz oder annähernd übernommen sind, als solche kenntlich gemacht und nach ihrer Herkunft unter Bezeichnung der Fundstelle einzeln nachgewiesen habe.

Ich erkläre des Weiteren, dass die hier vorgelegte Dissertation nicht in gleicher oder in ähnlicher Form bei einer anderen Stelle zur Erlangung eines akademischen Grades eingereicht wurde.

München, 01.12.2017

Ort, Datum

Unterschrift Doktorandin 